\title{
Dynamics of Suspended Dust Grains: Experimental Investigations and Implications for Protoplanetary Discs
}

\author{
Dissertation \\ for the award of the degree of Doctor of Philosophy (Ph. D.) \\ Division of Mathematics and Natural Sciences \\ of the Georg-August-Universität Göttingen \\ within the doctoral program \\ of the International Max Planck Research School (IMPRS) for \\ Physics of Biological and Complex Systems \\ and of the Georg-August University School of Science (GAUSS)
}

submitted by

Holly Capelo née Larson

from California, U.S.A.

Göttingen, 2017 


\section{Thesis Committee:}

Prof. Dr. Eberhard Bodenschatz

Laboratory for Fluid Dynamics, Pattern Formation, and Biocomplexity,

Max Planck Institute for Dynamics and Self-Organization

Prof. Dr. Annette Zippelius

Institut für Theoretische Physik, Georg-August-Universität Göttingen

Prof. Dr. Jens Niemeyer

Institut für Astrophysik, Georg-August-Universität Göttingen

\section{Members of the Examination Board:}

Referee: Prof. Dr. Eberhard Bodenschatz

Laboratory for Fluid Dynamics, Pattern Formation, and Biocomplexity, Max Planck Institute for Dynamics and Self-Organization

2nd Referee: Prof. Dr. Ansgar Reiners

Institut für Astrophysik, Georg-August-Universität Göttingen

\section{Further Members of the Examination Board:}

Prof. Dr. Annette Zippelius

Institut für Theoretische Physik, Georg-August-Universität Göttingen

Prof. Dr. Stefan Dreizler

Institut für Astrophysik, Georg-August-Universität Göttingen

Prof. Dr. Jens Niemeyer

Institut für Astrophysik, Georg-August-Universität Göttingen

Dr. Michael Wilczek

Max Planck Institute for Dynamics and Self-Organization

Date of oral examination: October 16, 2017 
$\begin{array}{ll}\text { Contents } & 5\end{array}$

$\begin{array}{ll}\text { Motivation } & 7\end{array}$

$\begin{array}{ll}\text { 1. Introduction and Theory } & \mathbf{1 4}\end{array}$

1.1. Composition of PPDs . . . . . . . . . . . . . . . . . . . . 14

1.2. Flow Regimes . . . . . . . . . . . . . . . . . . . . . . . 17

1.3. The Streaming Instability . . . . . . . . . . . . . . . . 26

2. Laboratory experiments of astrophysical flows $\mathbf{3 3}$

2.1. State of the Art . . . . . . . . . . . . . . . . . 33

2.2. A novel facility . . . . . . . . . . . . . . . 38

3. Experimental Methods 43

3.1. Flow Carrier Phase . . . . . . . . . . . . . . . . . . . . 46

3.2. Disperse Phase . . . . . . . . . . . . . . . . . . . . 74

3.3. Stereoscopic Reconstruction . . . . . . . . . . . . . . . . . . . 89

3.4. Tracking in Time . . . . . . . . . . . . . . . . 97

$\begin{array}{ll}\text { 4. Experimental Results } & 101\end{array}$

4.1. Data Characterisation . . . . . . . . . . . . . . . . . . . 101

4.2. Global statistical properties . . . . . . . . . . . . . . . . . 110

4.3. Particle dynamics on local subscales . . . . . . . . . . . . . . 116

4.4. Global mass-loading dependencies . . . . . . . . . . . . . . . 133 
5. Theoretical Results 137

5.1. Simulations . . . . . . . . . . . . . . . . . . . 137

5.2. Abstract . . . . . . . . . . . . . . . . . . . 139

5.3. Introduction . . . . . . . . . . . . . . . . . . 140

5.4. Mass-loaded particle rain . . . . . . . . . . . . . . . . . . . . . . 142

5.5. Numerical results showing spontaneous particle concentrations . . . . . . 148

5.6. Enhanced particle concentrations in protoplanetary discs . . . . . . . . 166

5.7. Future outlook . . . . . . . . . . . . . . . . . . . . . 172

5.8. Summary . . . . . . . . . . . . . . . . . 173

5.9. Linear stability analysis . . . . . . . . . . . . . . . . . . . 174

5.10. Toy model dispersion relation . . . . . . . . . . . . . . . . . . 177

5.11. Particle number test . . . . . . . . . . . . . . . . . . . . 180

6. Discussion and Perspectives 183

$\begin{array}{ll}\text { Appendix } & 191\end{array}$

A. Estimated parameters relevant to particle-turbulence interactions in PPDs . 191

B. Global Variation in Number Density . . . . . . . . . . . . . . . . . . . 197

C. Local Variation in Number Density . . . . . . . . . . . . . . . . . . . . 201

$\begin{array}{ll}\text { Bibliography } & 205\end{array}$

$\begin{array}{lr}\text { Curriculum Vitae } & 223\end{array}$

$\begin{array}{lr}\text { Acknowledgments } & 229\end{array}$ 


\section{Motivation}

In recent decades, we entered an era in which it is possible to observe planet-forming systems at several stages of their evolution, from the earliest dust- and gas-enshrouded objects that are collapsing to form stars, to the proto-planetary disc (PPD) phase, to a wide variety of extra-solar planetary systems (Seager \& Lissauer 2010). Planet formation is therefore known to be a ubiquitous and robust process and a natural byproduct of star formation. For many centuries, however, our own Solar System was the sole example of a planetary system (Copernicus 1543, Kepler et al. 1619, Galilei 1655, Le Verrier 1857, Schiaparelli 1867, Lowell 1908) and remains the only one that we can probe directly, such as by studying meteorites which arrive on the Earth's surface, transporting rocks back from the Moon and passing-by comets, and sending satellites to study the surfaces of comets and other planets (Papanastassiou \& Wasserburg 1971, Lebreton \& Matson 1992, Boss 1996, Flower 2016).

From our most-studied example, a general picture emerged, in which planets can be classified into two groups: the 'terrestrial planets', with their solid surfaces and thin atmospheres, reside close to the Sun, where refractory materials can remain solid at high temperatures, whereas the 'gas giants' possess atmospheres of indeterminate depth and reside beyond the radius at which liquids can freeze into ice. The favoured core-accretion formation model proposes that terrestrial and gas-giant planets form initially in the same way, where collisions 
between solid bodies, termed planetesimals, undergo a sufficient number of inelastic collisions to produce a bound object that becomes spherical under the effect of its own gravity (Morbidelli et al. 2012). The rocky core may differentiate and undergo geological processes such as those inherent to the Earth. Or, if the planetary core is above a critical mass, hydrostatic equilibrium in its atmosphere cannot be supported and runaway gas accretion occurs, leading to the production of planets such as Jupiter and Saturn (Mizuno 1980, Pollack et al. 1996). While this process is considered very plausible, in light of our good understanding of gravitational interactions and evidence for a collisional history amongst Solar-System bodies, it is still uncertain how planetesimals form in the first place.

As it ought to be hot closer to the star and colder at larger distances, there is necessarily a global radial thermal pressure gradient, $\nabla P$, in the PPD. In an equilibrium configuration, solid particles traveling in Keplerian orbits will resultantly dissipate momentum and drift radially due to drag from local gas that is rotating slightly more slowly under thermal pressure support, a point first considered by Whipple (1972). Even were it possible for particle-particle collisions to result in meter-sized particle aggregates, for solids of this size and typical material densities ( $\mathrm{a}$ few $\mathrm{g} / \mathrm{cm}^{3}$ ), the viscous coupling time is similar to an orbital time scale (order yr - $100 \mathrm{yr}$ ), implying that the disc will be depleted of planetesimal-forming material as meter-size ojbects fall unimpeeded into the central star. By pointing out that the radial drift rate peaked for solids of one meter, Weidenschilling (1977a) established the existence of a 'meter barrier' to planetesimal growth, which still poses the most stringent timescale constraints on formation mechanism theories. A successful theory must either cross or else bypass this barrier via rapid processes that occur over hundreds of years. This is quite short with respect to the multi-billion year evolution of a planetary system.

Particle radial drift occurs for reasonably inertial particles that must have already experienced collisional growth. The dynamics and hydrodynamics of colliding solid bodies, ranging in diameter from the smallest dust grains of a few $\mu \mathrm{m}$ to that of giant planets, are the 
key physical process underlying the creation of a mature planetary system such as our own. In order for the smallest particles in a PPD to encounter one another, the dust grains should exhibit peculiar velocities on top of their orbital dynamics. For particles below $\sim 100 \mu \mathrm{m}$, their relative velocities are due to Brownian motion (Einstein 1905), and charges on their surfaces can help attract particles towards one another (Heim et al. 1999, Kempf et al. 1999). But as agglomerates increase in mass, their inertia dominates over the effect of thermal velocity fluctuations, and similarly, over the electrostatic forces on their surfaces (Blum et al. 1996, 2000, 2006, Dominik et al. 2007, Blum \& Wurm 2008). The other mechanism that can cause excursions in the particles' trajectories are turbulent velocity fluctuations of the gas to which the particles are coupled, with collisions occuring at the interfaces between colliding eddies (Markiewicz et al. 1991, Cuzzi \& Weidenschilling 2006, Ormel \& Cuzzi 2007). Turbulence also drives gas accretion and, since dust grains are coupled to the gas, accretion flows provide yet another opportunity for particle-particle collisions.

Despite the various sources of particle relative velocity in PPDs, planetesimal growth models that rely solely on collisional growth do not match observed lifetimes of PPDs (Haisch et al. 2001, Wyatt 2008, Bell et al. 2013, Johansen et al. 2014). Moreover, the assumption of efficient particle-particle sticking is unrealistic and the lengthscale that can be reached via collisions is short of a meter. Controlled experiments conducted in vacuum and microgravity conditions (Dominik \& Tielens 1997, Blum et al. 1998, 1999, Blum \& Wurm 2000, Krause \& Blum 2004, Blum et al. 2014) demonstrate that porous solid agglomerates tend to fragment or bounce upon collision, once they accrue enough momentum. This leads to the proposition that the distribution of solid-body sizes in the early solar system peaks around diameters of several centimeters; in other words, it contains an abundance of small pebbles (Zsom et al. 2010, Güttler et al. 2010). Although the pebbles should eventually sediment into a thin layer, the density of such a layer is not considered high enough for gravitational energy to exceed the pressure supplied by the particle velocity dispersion and 
rotation. Particle-fluid interactions must therefore play an important role in advancing the evolution of the solid component towards producing planetesimals. Figure 0.1 summarizes the aforementioned physical mechanisms facilitating the growth of solid bodies in a PPD, as a function of particle size.

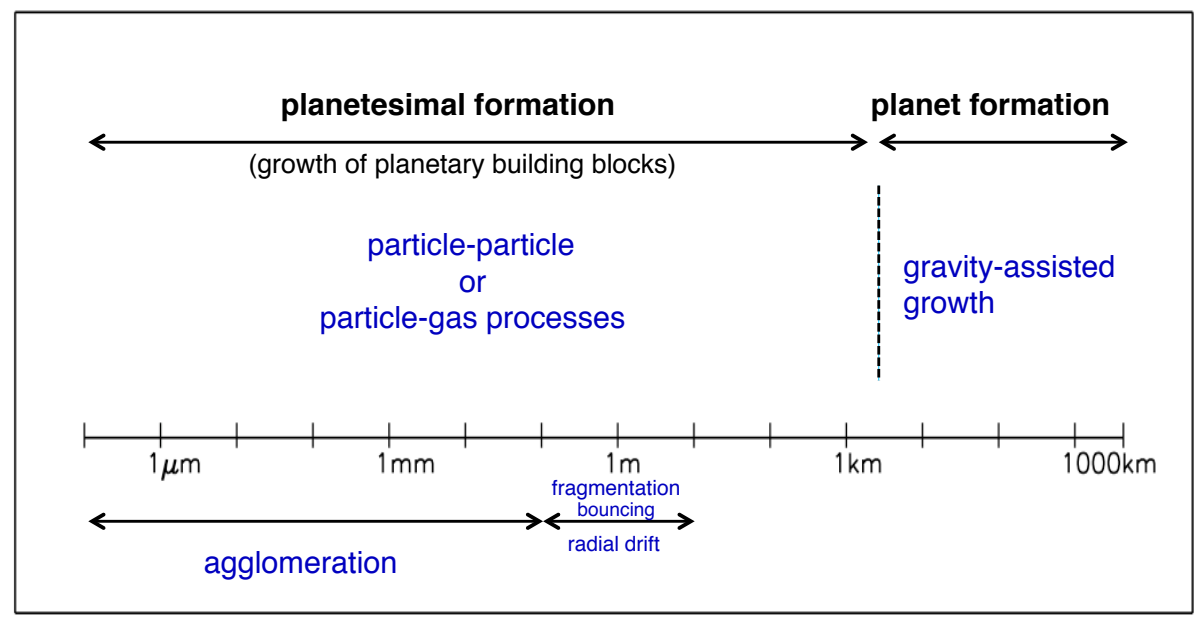

particle radius

Figure 0.1.: Dominant physical processes governing the growth of solids as a function of scale in PPDs, for typical solid densities of rock or ice. Dust-particle agglomeration by collisions is responsible for growth from the sub- $\mu \mathrm{m}$-decimeter range and gravity binds solids together at scales above $\mathrm{km}$ to form planets. In the intermediate size regime, particle-gas interactions must facilitate growth of planetesimals.

Depending upon the local conditions in specific regions of the disc, either turbulent or laminar gas flows may exist (Nakagawa et al. 1981, Weidenschilling 1980, Cuzzi et al. 1993, Barge \& Sommeria 1995, Nakagawa et al. 1986, Sekiya \& Nakagawa 1988, Weidenschilling 
\& Cuzzi 1993, Balbus \& Hawley 1991, Dubrulle et al. 1995, Tanga et al. 1996, Cuzzi et al. 2001a, Brandenburg \& Dobler 2002a, Hersant et al. 2005, Dubrulle et al. 2005, Johansen \& Klahr 2005, Johansen et al. 2006, Brauer et al. 2008a,c, Balbus 2009, Armitage 2011, Hughes et al. 2011). The fluid behaviour has a direct bearing on the fundamental stages of the evolution of the dust-particle component, including vertical sedimentation, the transport processes that lead to collisions, as well as the inevitable dissipation of particle orbital angular momentum due to viscous drag.

All of the above considerations point to the favorability of a multi-stage model of planetesimal formation in which dust grains can agglomerate by collisions up to the size of small pebbles, then undergo preferential concentration due to fluid-particle interactions, and finally gravitationally free-falling pebbles in the over-dense regions directly form a planetesimal (Weidenschilling 1995, Johansen et al. 2014, Testi et al. 2014). It is now considered probable that instabilities of the gas lead to local gas-pressure maxima which serve to concentrate solids.

Amongst a host of fluid instabilities thought to be present in PPDs, linear stability analysis and numerical simulations have shown the aerodynamic focusing of solid particles, referred to as the streaming instability (SI), to be an especially efficient means of creating sufficiently dense particle concentrations to form gravitationally bound planetesimals (Youdin \& Goodman 2005, Youdin \& Johansen 2007, Johansen \& Youdin 2007, Bai \& Stone 2010a,c, Johansen et al. 2009, 2012). An important advantage of SI is that it occurs rapidly enough to beat the timescale set by particle drift and it requires a relatively simple set of conditions, which were distilled by Youdin (2010) as :

- a global pressure gradient;

- local Keplerian dynamics;

- that the gas and particles are two-way coupled through drag forces. 
The requirement of Keplerian dynamics implies that the system is rotating. Jacquet et al. (2011) concerned themselves with the role of rotation in the SI and determined that the system is linearly stable when terms relating to rotation are ignored. Work presented in this thesis challenges this aspect of the theory, as simulations showed that the key features of the instability are present for a simplified version of the model, absent rotation, such as in the scenario of particles sedimenting under constant gravity. The numerical work regarding the simplified system has already been published in Lambrechts et al. (2016) and is presented in chapter 5 of this thesis ${ }^{1}$. The simple sedimentation experiment lends itself to an experimental investigation, which is the topic of the rest of the thesis. The goal in studying the system experimentally is to test the assumptions of the models leading to SI and to visit aspects not considered by theory, such as how particles in unstable regions interact with one another via the gas.

In the Introduction to this thesis, chapter 1, I first discuss the basic composition of PPDs, as is known from astronomy. I then describe the PPD flow conditions, with emphasis on the relevant features with respect to the gas-particle dynamical interactions. I discuss flow instabilities leading to turbulence in PPDs with explicit focus on the SI.

In chapter 2, Laboratory astrophysical flow experiment, I demonstrate that the previous state of the art in particle suspension studies cannot directly inform the understanding of PPDs, whereas the experimental facility that I built can. I include table and text previously published in Lambrechts et al. (2016) ${ }^{1}$.

In chapter 3, Experimental methods, I present the apparatus design principles and the measurement techniques I used. I report on the apparatus performance, including the gas

\footnotetext{
${ }^{1} \mathrm{My}$ own contribution to this work is the following: I independently wrote the text describing the laboratory facility; it is quoted verbatim in chapter 2 , section 2.2 of this thesis. The table in this section represents the culmination of extensive preliminary tests that I conducted in the experimental facility. This text benefited from editorial input from the other paper authors. I was involved in the early conception of the project framework, including extended discussions regarding key overlapping features between the dusty fluid model and the laboratory flow. I proofread several versions of the entire draft, including the results represented in chapter 5 of this thesis, checking the correctness of definitions, equations and language.
} 
flow calibration and characterization.

The Experimental Results are presented in chapter 4. The findings from 3D Lagrangian particle tracking data suggest the presence of a dusty-fluid instability in particle sedimentation experiments conducted under low gas-pressure conditions. I present the results of each dataset separately, then I consider results across datasets, as the primary control parameter - the mean dust-to-gas mass density ratio - varies.

The Theoretical Results in chapter 5 represent predictions on the presence of particle clumps in a model representing the experimental setup, previously published in Lambrechts et al. $(2016)^{1}$.

In chapter 6, I discuss the Conclusions to be drawn from the summary of results. I discuss possible implications and further avenues for the research presented herein. 


\section{CHAPTER 1}

\section{Introduction and Theory}

\subsection{Composition of PPDs}

The production of heavy elements in the late evolutionary stages of massive stars leads to a population of solid-phase silicate grains that first finds its way into the interstellar medium (ISM), and later into the circumstellar material of later-generation protostars (Kessler-Silacci et al. 2006). The gas, infused with such grains, distributes some of the giant molecular cloud's angular momentum and elongates into a PPD (Tscharnuter, W. M. et al. 2009, Bate \& Lorén-Aguilar 2017).

As is true of the Universe in general, PPDs are most abundant in Hydrogen and Helium. Owing to the $\mathrm{CNO}$ cycle in the interiors of an earlier generation of stars, the next most common elements are carbon, nitrogen and oxygen. Also to be found are refractory minerals, such as iron, magnesium and silicon, which are products of supernovae. Such are the primary materials available to compose planetary cores, and to do so, they should exist in their solid form. The fractional mass abundance of solid grains with respect to gas, I shall denote $\varepsilon$ and 
will refer to this quantity as either the 'condensible solid metallicity' or the dust-gas 'mass loading'. In each stage of stellar and planetary co-evolution, collisions between gas and solid grains (henceforth also 'dust' or 'particles') have a critical effect on the gas temperature and dynamics, since dust effectively absorbs and emits radiation as well as exchanges momentum with the gas. The research represented in this thesis focuses narrowly upon the momentum exchange between the gas and solid phases and how this process can mediate spontaneous particle-density enhancements on local scales in a PPD.

The mean free path of gas molecules in circumstellar contexts is large, yet so is the characteristic volume. It is therefore warranted to regard PPDs as a continuum fluid flow where the statistical mechanics of ideal gasses apply (Thompson 2006, Pringle \& King 2007). This can be true of both the gas and solid phases, and therefore a model of a PPD reduces to a set of definitions of its primative state variables.

\subsubsection{Equations of State}

Consider a reservoir mainly composed of gas, in orbit around a central stellar object, with thin vertical height with respect to its large radial extent. Its natural system of coordinates is cylindrical, containing a star at the origin, $Z$ being the distance above the orbital plane, $\Phi$ the azimuthal coordinate, and $R$ the radius. Initially in equilibrium, the cylindrical gaseous feature may not have finite physical boundaries, but is likely more well described by a diminishing density profile, in the $\hat{R}, \hat{Z}$, directions, and perhaps isotropic in the $\hat{\Phi}$ direction.

There are just three properties which fix the circumstellar-disk model, namely the temperature and surface density profiles, $T(R)$ and $\Sigma(R)$ respectively, and the mass of the star, $M_{\star}$. In their general form, these are expressed as follows:

$$
\Sigma(r)=\frac{M_{\star} H}{2 \pi R^{3}},
$$




$$
T(R) \propto R^{-c},
$$

where $\mathrm{H}$ is the disk scale height. Determing the power-law index, $c$, of the temperature profile requires assumptions upon the sources of cooling and heating in the disk, an estimate of the total mass of the system, and the central star's luminosity. The simplest estimate of $\mathrm{T}(\mathrm{R})$ is to assume that the disc is thin and therefore that its constituent particles achieve thermal equilibrium with their surroundings.

Written in terms of the equilibrium temperature at the radial position of Earth,

$$
T(R)=280 \mathrm{~K}\left(\frac{R}{\mathrm{AU}}\right)^{-0.5}\left(\frac{M}{M_{\star}}\right) .
$$

Note that the dependence on stellar mass comes from the fact that the luminosity of a star is proportional to its mass. Observed discs do not differ so much from this approximation, as it has been found that $c \sim 1$ (Dullemond et al. 2007). As in the hydrostatic equilibrium configuration of an atmosphere, the gas surface density in the $\pm \mathrm{Z}$ direction decreases from the midplane value $\Sigma_{m p}(R)$ with $\mathrm{H}$ as the e-folding factor and when reflected about the $Z$-axis takes a Gaussian form,

$$
\Sigma(Z, R)=\Sigma_{m p}(R) \exp (Z / H)^{2}
$$

It is common to constrain $\Sigma$ by requiring that its profile could result in our own Solar System, assuming that the planets form at their current location. This is done by distributing the measured masses of the planets in concentric annuli, corresponding to the distances between their nearly circular orbits ('minimum mass solar nebula', MMSN ${ }^{1}$. Weidenschilling (1977b), Hayashi et al. (1985)). Subscripting the gas, rock, and rock+ice surface density profiles, respectively, with $g, r$, and $r+i$,

\footnotetext{
${ }^{1}$ This model cannot describe the diversity of exoplanetary systems, but as of yet a more general model has not been found. See Raymond \& Cossou (2014) for discussion.
} 


$$
\begin{aligned}
& \Sigma_{r}(r)=7 \mathrm{~g} \mathrm{~cm}^{-2}\left(\frac{R}{\mathrm{AU}}\right)^{-3 / 2} \quad 0.35<r / \mathrm{AU}<2.7 \\
& \Sigma_{r+i}(r)=30 \mathrm{~g} \mathrm{~cm}^{-2}\left(\frac{R}{\mathrm{AU}}\right)^{-3 / 2} \quad 2.7<r / \mathrm{AU}<36, \\
& \Sigma_{g}(r)=1700 \mathrm{~g} \mathrm{~cm}^{-2}\left(\frac{R}{\mathrm{AU}}\right)^{-3 / 2} \quad 0.35<r / \mathrm{AU}<36 .
\end{aligned}
$$

From equations $1.5-1.7$, one can estimate $\varepsilon \simeq \frac{\Sigma_{g}}{\Sigma_{r}}$, or $\varepsilon \simeq \frac{\Sigma_{g}}{\Sigma_{r+i}}$, depending upon radius, which is at most 0.017 . This is of course a global estimate, as there are several mechanisms capable of creating local particle enrichments, particularly close to pressure maxima arising from fluid instabilities, which will be discussed in section 1.2.1.

The actual mass densities of observed PPDs are poorly constrained, primarily due to the fact that hydrogen atoms have no excitational transitions at the temperatures of PPDs, so the column densities cannot be determined from spectroscopy. The mass must be inferred from other less abundant species, such as $\mathrm{CO}$, which has low-frequency ro-vibrational transitions, corresponding to sub-millimeter wavelength radiation. Translating these measurements into hydrogen mass densities depends strongly on non-equilibrium chemistry models, which themselves are highly non-linear. Similarly, modeling of the spectral energy distributions of point sources is subject to model degeneracies in the radiative transfer calculations, which require a priori estimates of the line-of-sight density.

\subsection{Flow Regimes}

To create planetesimals, it is likely that the dust particles that are originally of $\mu \mathrm{m}$ size and homogeneously distributed throughout the fluid evolve through a sequence of stages closely related to the general scenario of Goldreich \& Ward (1973) or Safranov (1969); particles 
grow via collisional coagulation and concurrently sediment towards the midplane of the disc where, depending on the local density, a gravitational instability may facilitate the binding of a planetesimal. The particle layer that forms encounters a gas-velocity field governed by competing influences: orbital dynamics, a thermal pressure gradient originating from the central star, and differential rotation in both the radial and vertical directions. The gas itself may also be migrating due to accretion processes. In facing this velocity field, the particle will experience viscous drag, in a form that depends upon the particle size, $d_{p}$, by comparison to the mean-free path of the gas $\lambda$, referred to as the Knudsen number,

$$
K n \equiv \lambda / d_{p}
$$

For a particle whose velocity relative to the gas is $\delta u$, when $d_{p}$ is larger than $\sim \lambda$, the 'Stokes' drag law, which depends mainly on dynamic viscosity, $\eta$, applies. However, when the particle size is small compared to the mean free path of the gas, the 'Epstein' drag force (Epstein 1924) arises due to collisions with individual freely-flowing molecules, and therefore there is a dependence upon the gas density density, $\rho_{g}$, as well as the mean thermal velocity, $v_{\text {therm }}$, of the gas molecules:

$$
F_{D}= \begin{cases}3 \pi d_{p} \eta \delta u & \text { Stokes } \\ \frac{\pi}{3} \rho_{g} d_{p}^{2} v_{\text {therm }} \delta u & \text { Epstein }\end{cases}
$$

Equating these two expressions, the transition between these two regimes occurs for a particle diameter $d_{p}=9 / 2 \lambda$. Although this piece-wise function is often used to mark the drag regime transition in astrophysical applications, this approach necessitates the caveat that the transition is not so steep, as there are intermediate regimes between the two, which I delineate in more detail in chapter 4. At one astronomical unit (AU), this transition occurs 
for a particle diameter of approximately $6.4 \mathrm{~cm}$ (Johansen et al. 2014). This also happens to be very close to the maximum size to which dust particles can grow by collisions: the regime in which particle-fluid interactions must be the dominant concentration mechanism. A comparison of the momentum of a particle with mass $m$ to its drag force gives the relaxation time, on occasion also referred to as the particle 'stopping time' or 'friction time',

$$
T_{f}=\frac{m|\delta u|}{F_{D}} .
$$

Generally, this is the scale time over which particles couple to a flow after being accelerated. Accordingly, it is the scale time over which a settling particle approaches its constant 'terminal velocity', $u_{t}$, with respect to the velocity of the viscous medium that it encounters. Under constant gravitational acceleration $g$, the two quantities are related:

$$
u_{t}=T_{f} g
$$

For a low-mass particle located at an arbitrary cylindrical distance $R$ from a star of mass $\mathrm{M}_{\star}$, the elliptical Keplerian orbit has negligible eccentricity and the gravitational acceleration, $G M_{\star} / R^{2}$, G being the gravitational constant, is balanced by the circular centripetal acceleration $v^{2} / R$, giving the Keplerian velocity $v_{K} \equiv \sqrt{G M_{\star} / R}=\Omega R$. The angular velocity $\Omega$ is used to define the friction time $\tau_{s}$ as a dimensionless quantity:

$$
\tau_{s}=\Omega_{K} T_{f}
$$

which may also be refered to as the Stokes number, St. Assuming that Solar System solids all have on average the material density of rock, $\approx 3 \mathrm{~g} / \mathrm{cm}^{-3}$, allows one to parameterise the particle size and specific gas conditions via $\tau_{s}$. The primitive state variables, such as pressure $\mathrm{P}$ and temperature $\mathrm{T}$ as well as the viscosity are subsumed into this relation through 
$F_{D}$, since $v_{\text {therm }}$ is approximately ${ }^{2}$ the sound speed,

$$
c_{s}=\sqrt{\frac{\partial P}{\partial \rho_{g a s}}} .
$$

In physical units, $c_{s}=k_{B} T / \mu m_{p}$, where $k_{B}$ is Boltzman's gas constant, $\mu$ is the mean molecular mass in units of the proton mass ${ }^{3}, m_{p}$. The choice of $\mu$ depends upon estimates of cosmic abundances and the assumed amount of chemical enrichment of the protostellar nebula. One generically thinks of the Stokes number, St, as a comparison of the viscous time scale to the typical velocity gradient (due to either shear or strain) in the fluid. As such, equation 1.12 represents a Stokes number, since $\Omega_{K}(R)$ is an expression of shearing rate in a differentially rotating disk.

Just as the gas is treated as a continuum, it is common to describe the particle population this way as well. Making an analogy of the granular particles to that of gas molecules, the particles' velocity dispersion $\sigma$ stands in for the particle sound speed.

The motions in a PPD are in large part subsonic, or possibly transonic, expressed by the Mach number,

$$
M a=U / c_{s}
$$

being $\leq 1$, where $U$ is the characteristic velocity. An exception would be in areas where the gas accretion rate is high enough, or pressure gradients steep enough, to lead to supersonic shocks, but I am not studying such processes here. Therefore, the flow velocity field of the fluid will be described by the incompressible Navier-Stokes equations, expressing continuity of mass:

$$
\nabla \cdot \mathbf{U}=0
$$

\footnotetext{
${ }^{2}$ Sources vary on which definition to use, but they all result in prefactors that vary to within a factor of one of the sound speed.

${ }^{3}$ The proton mass is convenient when hydrogen is the most abundant species, but I will also use $c_{s}=\sqrt{\frac{R T}{\mu N_{A}}}$, where $\mathrm{R}$ is the molar gas constant, $N_{A}$ Avagadro's number and the molecular mass of the gas is given in moles.
} 
and momentum balance,

$$
\overbrace{\rho\left[\frac{\partial u}{\partial t}+(\mathbf{u} \cdot \nabla) \mathbf{u}\right]}^{\text {local }+ \text { convective accelerations }}=\underbrace{\mathbf{f}}_{\text {body forces }}-\overbrace{\nabla P}^{\text {absolute pressure }}+\underbrace{v \nabla^{2} \mathbf{u}}_{\text {viscous stress }} .
$$

By 'absolute pressure' I am referring to the fact that one often includes conservative forces in addition to the gas pressure in this term; the other labels are self explanatory. Depending upon the regime of the flow, certain simplifications can be made to this equation. The hydrodynamic stability of a fluid, which can be seen as how effectively viscosity damps inertial motions, is uniquely determined by its dimensionless Reynold's number, which compares the characteristic length $L$, velocity $U$, and kinematic viscosity $v=\eta / \rho_{g}$ :

$$
R e=\frac{U L}{v} \sim \frac{|\rho(\mathbf{u} \cdot \nabla) \mathbf{u}|}{\left|\eta \nabla^{2} \mathbf{u}\right|}
$$

We see that, in the limit of very low $R e$, the velocity fluctuations are damped by viscosity and the convective acceleration on the left-hand side of equation 1.16 can be ignored. Conversely, if $R e$ approaches infinity, the flow can be considered inviscid (Pringle \& King 2007, Yaglom \& Frisch 2012). Provided that Re (and $S t$ ) are small for the length and time scales under consideration, then the left hand side of equation 1.16 can be set equal to zero, yielding the Stokes equations, which have special properties; importantly, they are linear, and the viscous forces upon an obstacle in the flow can be described by equation 1.9. They also contain several important symmetries, such as invariance to translation in space and time. The Navier-Stokes equations possess Galilean invariance for rectilinear accelerations. They are not generally frame invariant under rotations. Keplerian rotation, with small corrections due to $\nabla P$, is often assumed of a PPD, and I assume the same for simplicity.

For the most general case of a rotating disc, the body forces will include the Coriolis force and one quantifies the relative importance of global rotation with respect to local inertial 
circulation via the Rossby Number:

$$
R o=\frac{U}{L|\omega|} \sim \frac{\mathbf{u} \cdot \nabla \mathbf{u}}{\Omega \times \mathbf{u}}
$$

By studying fluid flows over the dust friction time scale and associated length scale, one works in the high Ro regime; this is one reason why the experiments presented in this thesis do not need to be rotating (explained further in chapter 5). Similarly, one often treats the particle-gas mixture as a laminar flow, despite the fact that PPDs are presumed to be turbulent, a fact towards which I now turn the attention.

\subsubsection{Fluid instabilities leading to turbulence in PPDs}

PPDs fall into the general category of rotating, cylindrical, astrophysical objects known as accretion disks, which are also found around compact stellar remnants or the supermassive black holes at the centers of galaxies. Accretion is the most efficient known mechanism for converting energy into radiation and is required to explain the high observed luminosities and presence of high-frequency photons (UV and X-rays) observed in this class of systems. Although molecular viscosity is not thought to play a role in the orbital angular momentum dissipation that drives accretion of gas onto the star, the dimensional form of Re makes an appearance in the parameter characterising anomalous viscosity due to turbulent shear, $v_{\text {anom }}=\alpha c_{s} H$, first introduced by Shakura \& Sunyaev (1973).

The possible causes of turbulence include the magneto-rotational instability (MRI) and a number of additional flow instabilities mentioned below. The conditions for the MRI to occur is that the ionization level is high enough for stellar magnetic fields to remain well coupled to the disk. Since these conditions are not met in all places at once, there are believed to be regions, particularly near the disk mid-plane, where the flow is laminar or weakly wave-like, however still subject to vertical shear instabilities leading to turbulence, or the 
Kelvin-Helmholtz instability arising from the enhanced density gradient of the dust disk.

A linear particle-drag law is generally not warranted if the flow conditions are turbulent, should the velocity gradient due to turbulent motions outpace the viscous relaxation time. This is determined by the value of St with respect to the Kolmogorov time, $\tau_{\eta}$, which is the typical turnover time of the smallest turbulent eddy, according to the energy casdade picture proposed by Kolmogorov (1941), in which velocity fluctuations due to energy injection at large scales are damped at increasingly smaller eddy size scales, at an energy dissipation rate $\varepsilon^{\prime}$. For standard disk turbulence models, the smallest (dissipation) length scale of the energy cascade, the Komogorov length $\eta^{\prime}$, is of the order of a km (Cuzzi \& Weidenschilling 2006). The turnover of even the smallest eddies at this scale is longer than the coupling time of dust grains, therefore it is generally considered valid to study the dust-gas fluid coupling as a laminar, however time varying, flow. To reassert the validity of the laminar flow assumption, Appendix A presents an analysis of the range of St in a typical PPD model.

The presence of a particle in a fluid is known, under some conditions, to generate smallscale turbulence, in which case the use of either of the above drag laws would not be valid. Whether or not a wake develops is determined by the particle-scale Reynold's number $R e_{p}$ that is calculated using $d_{p}$ as the characteristic length. A wake should not develop for $R e_{p}<<1$. Note that, since the interplanetary medium has low $\rho_{\text {gas }}, R e_{p}$ is naturally low for small particles.

It is common to assume that a hydrodynamic instability proceeds first through a linear onset stage, given that the higher order terms in the evolution equations become more important as perturbations grow in amplitude (Drazin \& Reid 2004). Applying the method of normal modes, described at length below, one can arrive at a number of well-known conditions for hydrodynamic stability of an accretion disk, including but not limited to the Rayleigh criterion i.e. that angular momentum increases with radius, the condition for the onset of the MRI (performed in Chapter 3 of Armitage (2010)), or the baroclinic instability, which arises 
from a misalignment of the pressure and density gradients (Klahr 2004). Although it is not the approach taken in the canonical work of Toomre (1964), one can also use this approach to re-derive the commonly used Q parameter for the onset of gravitational instability,

$$
Q \equiv \frac{c_{s} \Omega}{\pi G \Sigma}
$$

which illustrates a competition between thermal and rotational pressure against gravity and the disk becomes unstable once $Q<1$. A high local density can drive $Q$ downwards as can low temperatures. Disk fragmentation due to gravitational instability is one mechanism to drive disk turbulence. However, this work is concerned not with gravitational or sheer instabilities leading to turbulence, such as those mentioned above, but rather with the proposition that aerodynamic drag-driven instabilities of the particle-fluid mixture can concentrate particles sufficiently for a gravitational instability to occur and produce a planetesimal, with emphasis on the former mechanism. In what follows, I describe the general procedure of stability analysis and report on notable results when applied to the particle-gas mixture where the two components are coupled via aerodynamic drag. Note, however, that although this is typically the first approach in understanding the stability of a system, it is not the only method (see e.g. Cross \& Greenside (2009)).

Assume that a translationally invariant medium, i.e. uniform in at least one (perhaps spatial) direction, has either infinite or periodic boundaries. Once finding the explicit evolution equations that describe the system, the number of parameters can be reduced by recasting the equations in dimensionless form. If there is at least one base state, which is time-independent with respect to the coordinates of the invariant (spatially extended) direction, the evolution equations can then be linearized about such state to obtain a new set of linear equations for an infinitesimal perturbation to the base state.

The question is simply whether the perturbation field between a nearby solution and the 
base state will amplify with time. The perturbations are assumed to be periodic and solutions to this set of equations to take the form of an exponential Fourier mode. Owing to the linearity of the equations, a general solution is given by a superposition of the particular solutions ${ }^{4}$, that is $p(x, t)=\Sigma_{k} c_{k} e^{\sigma_{k} t} e^{i k x}$, where $c_{k}$ and $\sigma_{k}$ are complex coefficients and the growth rate, respectively, which depend upon the wavenumber, $k$. Substituting such a solution into the linearized differential evolution equations (and considering the boundary conditions) for the perturbed system, the growth rate (of each mode) becomes a complex-valued, multiplicative coefficient. Setting the determinant of the resultant coefficient matrix to zero produces a dispersion relation, which relates $\sigma_{k}$ to $k$. Examining the sign of the real part of the growth rate then indicates whether a given perturbed mode will increase or else decay exponentially. Should the growth rate possess a non-zero imaginary part, then a bifurcation occurs and the instability is said to be oscillatory. Parameter values which change the behavior of the growth are said to be critical parameters. The critical wave number determines the length scale of the perturbation, and is given by the magnitude of the wave vector for which the maximum of the real part of the growth rate becomes positive.

Regarding the evolution of an instability, one expects the non-linearities to quench the exponential growth and for the state to reach a stationary, stable state. For instabilities which are said to be oscillatory, one expects for the saturated nonlinear state to depend periodically on time and to be a state of nonlinear oscillations or waves; this type of instability predicts the possibility of spatial structure on a length scale above the critical threshold.

\footnotetext{
${ }^{4}$ summed when there are periodic boundary conditions, giving infinite discreet solutions, and integrated when the boundaries are infinite.
} 


\subsection{The Streaming Instability}

In the model that produces SI, the explicit evolution equations for both the gas and particles are continuum fluid equations ${ }^{5}$. The velocity fields of each phase, $\mathbf{v}_{g}$ and $\mathbf{v}_{p}$, for the gas and particles respectively, are represented by a special case of equation 1.16, written with the Lagrangian material derivative:

$$
\begin{array}{r}
\frac{\partial \rho_{p}}{\partial t}+\nabla \cdot\left(\rho_{p} \mathbf{v}_{p}\right)=0 \\
\frac{D \mathbf{v}_{p}}{D t}=-\Omega_{K}^{2} \mathbf{r}-\frac{1}{T_{f}}\left(\mathbf{v}_{p}-\mathbf{v}_{g}\right) \\
\nabla \cdot \mathbf{v}_{g}=0 \\
\frac{D \mathbf{v}_{g}}{D t}=-\Omega_{K}^{2} \mathbf{r}-\frac{\nabla P}{\rho_{g}}+\frac{\varepsilon}{T_{f}}\left(\mathbf{v}_{p}-\mathbf{v}_{g}\right) .
\end{array}
$$

Here $\mathbf{r}$ and $\mathbf{v}$ are vectors. Using this formulation, one assumes the dust to be 'pressureless' not only in the sense that it does not respond to the global pressure gradients, but also that the relative motions are damped and so the dusty fluid has no 'temperature'. The componentwise solutions, written in terms of $\tau_{s}$, are presented in Nakagawa et al. (1981) and references thereto. In the Stokes flow approximation, the difference in velocity between the particle and gas components of the disc is:

$$
\delta \mathbf{U}=\mathbf{v}_{p}-\mathbf{v}_{g}=\frac{\nabla P}{\rho_{t o t}} T_{f}
$$

where the total density $\rho_{t o t}=\rho_{g}+\rho_{p}$. Note the role of the pressure gradient in driving

\footnotetext{
${ }^{5}$ As the conditions at various locations in PPDs span a wide range, it is in most cases practical to focus on specific regions of the disc, i.e. limited to a specified radius. The shearing-sheet approach considers a local patch of the disc that is small enough that curvature is neglected, allowing the equations of motion in cylindrical coordinates to be recast into a Cartesian frame. The model is then a simple one, representing a parallel shear flow, where velocities are measured relative to the local Keplerian velocity. The boundaries are typically taken to be periodic. The primitive variables of the model are given in section 1.1.1 of this thesis.
} 
the differential motion between the two phases and that the existence of two-way drag-force coupling depends upon its presence. It is from this relation that the system derives the free energy to move from equilibrium to instability Youdin (2010), Lin \& Youdin (2017).

Youdin \& Goodman (2005) performed stability analysis by the method of normal modes on this system of equations, using the Stokes flow approximation ${ }^{6}$ to linearise the equations and assuming axial symmetry in the disc plane. They found an unstable radial mode, i.e. in the direction of particle radial drift. The fastest growth occured for the control parameters $\tau_{s}$ and $\varepsilon$ each $\gtrsim 1$. Physically, this corresponds to a situation where the particle-gas mass loading is pre-enhanced from its canonical average value of 0.01 , which may occur due to local pre-enhancement by pressure over-densities or gas evaporation. This value of the friction time corresponds to particles that are moderately coupled to the disc: neither passive tracers of the gas nor ballistic objects that easily plow through it. They showed that the rate of the density perturbation growth was faster than particle drift and that the amount of mass contained in a length-scale defined by the radial wavelength was sufficient to produce a $100 \mathrm{~km}$ planetesimal. In this work, they also posed an analogy between the onset of this instability and that of developing turbulence in pipe flows, via Howard's semi-circle theorem (Drazin \& Reid 2004, Yaglom \& Frisch 2012).

Johansen \& Youdin (2007) numerically evolved the non-linear equations of motion in the sheering-box approximation and confirmed that the instability resulted in a saturated oscillatory state, with particle clumping size scales roughly consistent with what predicted in the analytical studies of Youdin \& Goodman (2005) and Youdin \& Johansen (2007). They also confirmed that the assumption of an incompressible gas was valid, since, although the particle field exhibits density enhancements, the gas appears not to do so, meaning that pressure gradients are readily smoothed out. Johansen et al. (2007) allowed for both disc turbulence and particle self gravity in their simulations and confirmed that planetesimal

\footnotetext{
${ }^{6}$ Which they refer to as the 'terminal velocity approximation'.
} 
formation via SI results in correct planetesimal size and mass distributions and occurs quickly enough to beat the timeline set by radial drift.

A noted feature of the SI is apparent collective-particle drag reduction: once clumping occurs due to the instability, the density enhancements are augmented because groups of particles travel faster as a pack than they do in isolation. In this way, they can catch up to particles which are leading in their orbit and gather particles that are drifting inward. The run-away nature of the instability is often compared to bicycle riders in a peloton, however Youdin \& Johansen (2007) make the distinction that the 'drafting' effect is not due to attractive wakes forming behind the particles (after all how could it be, since their $R e_{p}$ is low), but rather because the collective inertia of the particles slows the gas flow around them. It should be noted that the tendency for particles to 'catch up' to others is not thought to be the cause of the instability at onset, but it is a symptom of the system exhibiting the instability and it plays a role in augmenting density wave amplitudes. Figure 1.1 presents a simplistic schematic of how the reduced energy dissipation due to collective particle drag back-reaction influences the outcome for particles that would otherwise simply drift into the star. Dust grains pile up and produce regions sufficiently dense for gravitational interactions between the particles to become appreciable. 

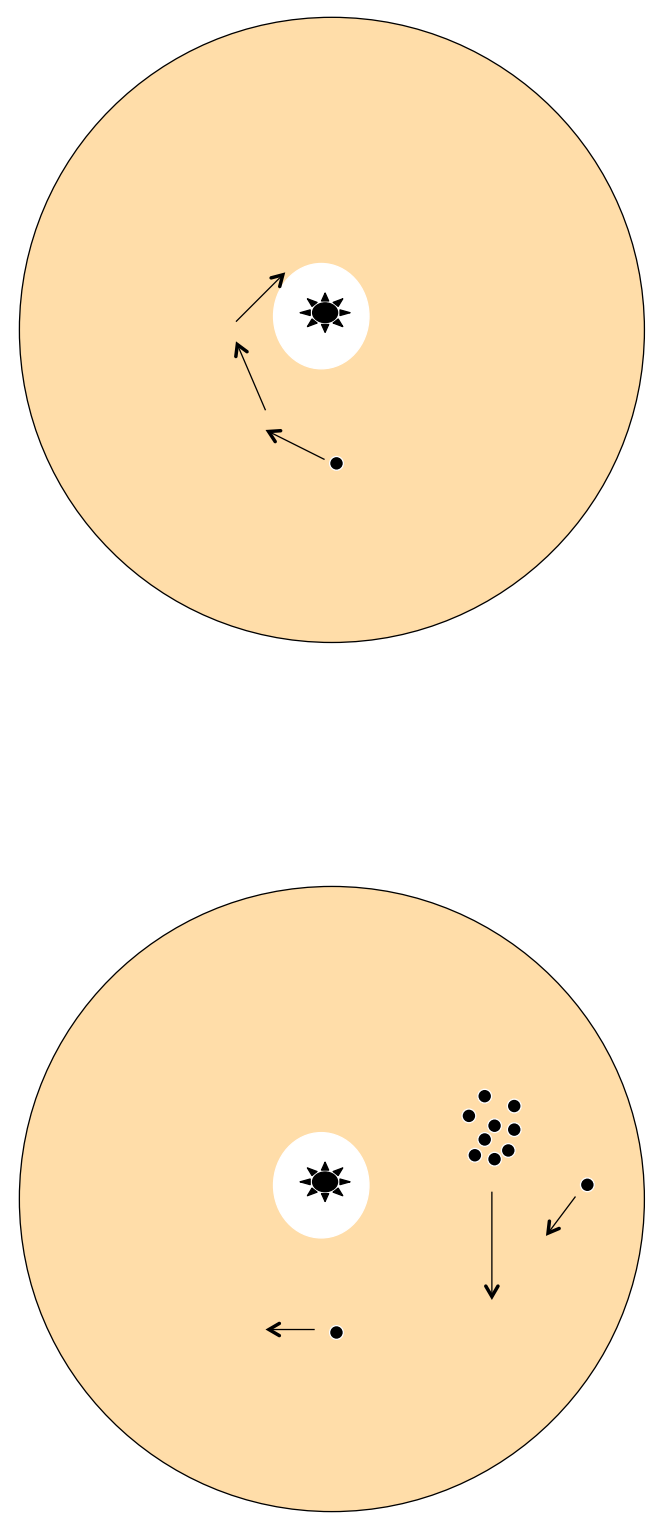

Figure 1.1.: Alternative scenarios for the fate of solid particles in a PPD, imagined to be face-on, with the star at the center. On the top, particles dissipate momentun and spiral into the star. On the bottom, clusters of particles accumulate in piles and collectively resist energy dissipation by drag forces; the cluster maintains its orbit and continues growing by catching up to particles that it finds in its path and gathering particles that are drifting inward. 
Although a criticism of the above mentioned studies is that the required value of $\varepsilon$ is rather high, Johansen et al. (2009) turned this reasoning around, to claim that the high-metallicity requirement is consistent with observations of extra-solar planets that show a bias towards having formed around super-solar-metallicity stars (Fischer \& Valenti 2005).

Bai \& Stone $(2010 a)$ repeated the numerical study and proved that the SI is robust to turbulence generated by Kelvin-Helmholtz instabilities in the disc midplane. In Bai \& Stone (2010c), they emphasised the role of the global pressure gradient, and found that varying the strength of the gradient changed the outcome for particle clumping.

Since, other works have extended studies of the SI to include it in planetesimal formation theories including dust coagulation (Dra̧żkowska \& Dullemond 2014), or to derive the initial mass function of planetesimals (Simon et al. 2017).
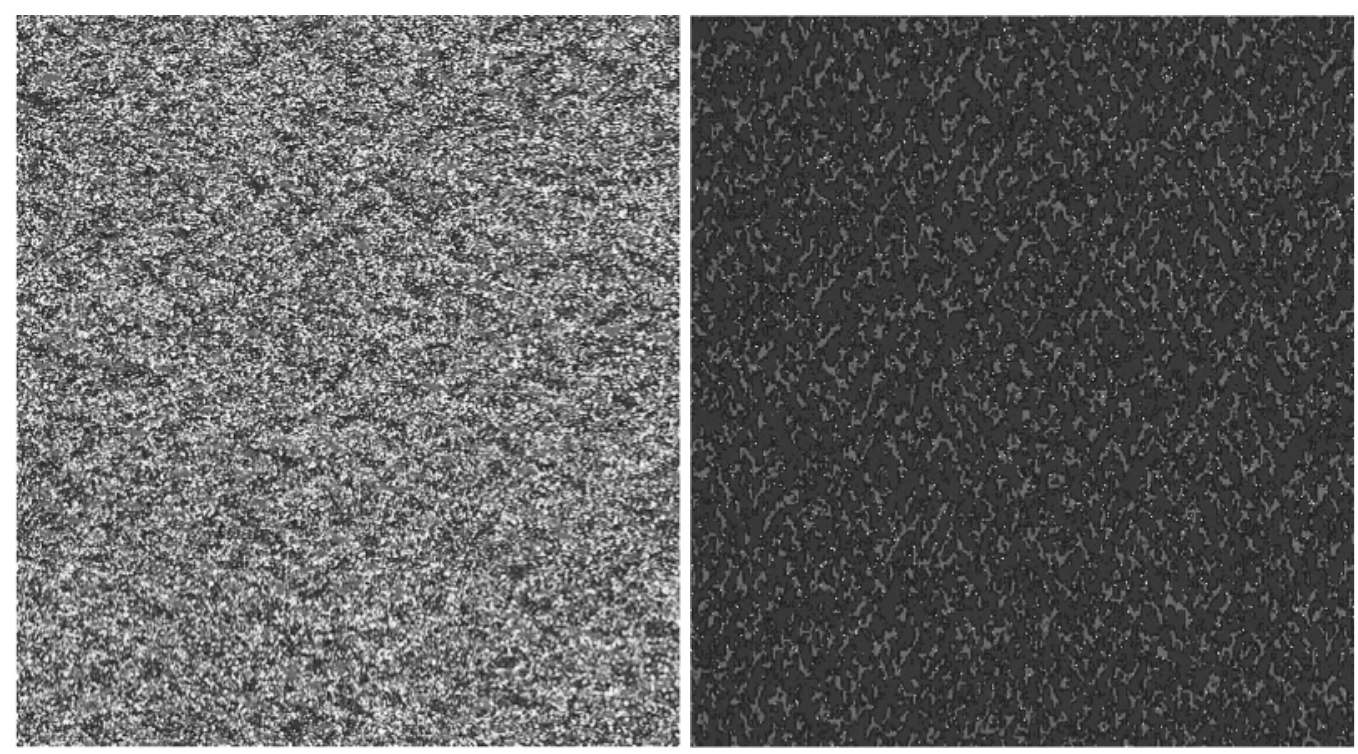

Figure 1.2.: Particle density maps in a local patch of a Keplerian PPD with mass loading 1 on the left and mass loading 10 on the right.

Each of the numerical studies, as in the analytical work, find $\varepsilon$ to control the instability growth rates. In figure 1.2, I show low-resolution snapshots of shearing sheet simulations of the SI, performed with the same numerical tool as was used in Bai \& Stone (2010b), 
the publicly available hydrodynamic grid code Athena ${ }^{7}$. To emphasise the role of the mass loading, I compare density maps of the disc midplane just a few time steps (in code units) after initialization for mass loading 1 and mass loading 10 . The particle mass density spectrum is shown in greyscale. While the mass loading 1 case shows some noise with a few enhancements, the mass loading ten case shows that the particle have completely differentiated from the gas.

The same attributes as those present due to SI, namely the dependency of particle clumping on $\varepsilon$ and apparent collective particle-drag reduction, were found in simulations of a greatly simplified system, involving only the sedimentation of particles that are originally homogeneously mixed with the gas (published in Lambrechts et al. (2016), and reproduced in chapter 5).

While the SI arises from the two-fluid, drag-coupled model equations presented above, more recently, Lin \& Youdin (2017) offered a more general single-fluid model in which the diffusion of dust grains is treated analoguously to entropy gradients in a dust-free adiabatic gas with heat or cooling sources applied. They study the SI with this formalism and find that dust overdensities are necessarily followed by lagging gas-pressure waves. They demonstrate, when dust is treated in the thermodynamic limit, that particle densities grow in amplitude as positive work is performed on the system. This interpretation reasserts the role of the particle-gas relative velocity, arising as particle radial drift due to the pressure gradient in the orbital plane or due to particle sedimentation in the vertical direction, in driving the instability.

Johansen et al. (2014) provide a comprehensive overview of the range of fluid instabilities than can lead to turbulence in PPDs, with a map showing which radial locations are favorable for their occurence. In addition to the efficiency of the SI in forming particle clumps quickly,

\footnotetext{
${ }^{7}$ I ran these simulations on the University of California computer cluster Hyades during the program HIPAC International Summer School on AstroComputing, Star and Planet formation. The numerical module can be downloaded as a test case in Athena 4.2 under /tst/particles.
} 
the conditions for its onset are met at the orbital radius where terrestrial planets can form in situ, within the radii of one to ten AU.

Observed PPDs are optically thick and difficult to resolve ${ }^{8}$, posing challenges to directly studying the system dynamics at dust-grain scales. While promising, the SI is thus far a mathematical and numerical phenomenon that is model dependent. The original work in the present thesis represents the first approach to understanding the SI from an experimental physics perspective.

\footnotetext{
${ }^{8}$ Despite impressive recent achievements in long-baseline interferometry with the ALMA observatory.
} 


\section{CHAPTER 2}

\section{Laboratory experiments of}

\section{astrophysical flows}

In this chapter, I will introduce the experimental facility, highlighting the features that make it appropriate for studying dust-gas mixtures in a PPD. First, I establish some important previous findings in the field of particle-laden flows.

\subsection{State of the Art}

The model leading to SI (henceforth unstable 'dusty-fluid' model) consists of a laminar, incompressible, two-phase flow. $R e_{p}$ is small enough that turbulent wakes will not develop around the particles and a linear drag law applies. The periodic in space and time particledensity distribution occurs for particle-gas mass loading of order unity and for moderately intertial particles.

Such a description resembles low $R e_{p}$ particles sedimenting in a fluid, and therefore 
an overview of this type of system is a natural starting point for comparison. Particle suspensions (for relatively buoyant particles) and fluidized beds (for particles requiring a counterflow to remain suspended against the gravitational field) have been studied extensively for their applications in engineering and hydrology as well as to understand their fundamental properties (Guazzelli et al. 2011). Although there are some important differences between suspensions and fluidized beds, I will refer to them collectively as sedimentation vessels and their contents as two-phase flow, unless otherwise stated. In particle-laden two-phase flows, one calls the gas or liquid fluid the 'carrier phase' and the particulate matter that infuses it the 'disperse phase', and I sometimes adopt the same terminology. The classification of these systems depends upon $\varepsilon$ and the volumetric filling factor $\phi$, where two-way drag coupling is considered important around $\varepsilon \sim 1$. With increasing $\phi$, the collisional and perturbative dynamics of the particles become relevant (Balachandar \& Eaton 2010, Marchioli 2017). Similarly in the case of PPDs, the two-way drag coupling equation is appropriate, until particle densities become too high (Johansen et al. 2012).

A striking feature of sedimentation is that the disperse phase exhibits density and velocity variations (Batchelor 1972, Nicolai et al. 1995, Feng et al. 1994, Uhlmann \& Doychev 2014). The fluctuations, $\sigma$, within a region of length $l$, are mediated by a balance between variations in the mean number density, $n$, due to poisson statistical fluctuations, and Stokes drag:

$$
\sigma=O\left(\frac{m_{b} g \sqrt{n l^{3}}}{6 \pi \eta l}\right)=O\left(u_{t} \sqrt{\frac{\phi l}{r_{p}}}\right)
$$

where the volumetric filling factor is $\phi, \sqrt{n l^{3}}=\Delta N$ is the variation of $n$ in the region and $m_{b}=\Delta N \frac{4}{3} \pi l^{3} \Delta \rho g$ is the mass of the particle compensated for buoyancy. The difference in mass density between the carrier and disperse phases is $\Delta \rho{ }^{1}$. Fluctuations occur about a mean settling velocity, which is itself modified by the presence of both the fluctuations and a

\footnotetext{
${ }^{1}$ The densities under comparison are not the same as those for $\varepsilon$, since here it is the material density of the substance, whereas $\varepsilon$ is the ratio of the spatially averaged densities and depends upon $n$.
} 
backflow of the carrier phase that is displaced by the sedimenting spheres (Batchelor 1972). Ignoring the effect of polydispersity, the mean sedimentation velocity of a particle is

$$
\left\langle v_{\text {sed }}\right\rangle=u_{t}(1-6.55 \phi)
$$

From this expression, one sees that the mean settling velocity is hindered with respect to its terminal velocity for appreciable values of $\phi$. Relation 2.1 was determined to hold experimentally up to the correlation length $\xi=l$, which is determined by finding the first minimum of the autocorrelation function of the velocity fluctuations (Tee et al. 2008). Clumps of spheres defined by the length $\xi$ are advected with a correlated velocity fluctuation, until the clump is either diffused, with diffusivity ${ }^{2} D=\xi \sigma$, by particle-particle interactions or the constituent particles encounter another region with which their velocities become correlated. The velocity fluctuations are found to depend upon $\phi$. In simulations and experiments of particle suspensions, Kalthoff et al. (1995) showed that $\sigma$ increases from $0<\phi<0.3$ but this dependence then declines. The explanation being that the interparticle forces that serve to enhance the velocity dispersion at moderate filling factors eventually suppress fluctuations at higher particle densities, since the interactions become dissipative.

In addition to statistical density fluctuations, certain systems show local density enhancements in excess of $\Delta N$. However, this effect is strongest for non-spherical objects such as spheroids or granular rods, and the tendency of the particles to form overdensities is partially explained by their preferential orientation towards high-density regions (Saintillan et al. 2006, Narayan et al. 2007, Guazzelli \& Hinch 2011, Niazi Ardekani et al. 2016).

Other particle-pair phenomena can influence the relative velocities of the particles. In particular, wake attraction is an important case (Fortes et al. 1987), in which particles can form a low-pressure region that draws particles into it, a phenomenon also colloquially

\footnotetext{
${ }^{2}$ alternatively, defined in terms of wave number $k$ and the decay scale time $\tau(k): \tau(k)^{-1}=D k^{2}$.
} 
referred to as "drafting". This effect is commonly evoked to explain the SI, although it is only a very qualitative analogy since wake attraction describes typically two-particle dynamics of moderate $R e_{p}$ systems.

The presence of the disperse phase can alter the properties of the carrier phase. For very high $\phi$, as in a packed granular bed, it has long been noted that the pressure of the fluid decreases and that the pressure differential depends upon the average free opening area and the thickness of the particle layer. Partly for historical reasons ${ }^{3}$, the pressure difference is described by empirical relations such as Darcy's law (explained in the translation by Bobeck (2006) of Darcy's original work) or similarly, the Carman correlation. These laws remain useful because equation 1.16 is challenging to solve in three dimensions when there are many obstacles in the flow, particularly for high Re. Because particles are a source of stress on the fluid, they can have a resultant effect on the carrier fluid's viscosity and also the drag force.

While the mechanism thought to be responsible for the coordinated motions of particles in sedimentation vessels are the long-range hydrodynamic interactions between particles, this effect is neglected in the dust-fluid models of PPDs. It is warranted, given that the mean interparticle separation is long, and so the fluid accelerations due to the particles ought to decay on length scales shorter than the particles' separations . Guazzelli \& Hinch (2011) provide a particularly general definition of the Stokes number:

$$
S t=\frac{d_{p}^{2}}{t \eta} \sim \frac{|\partial \mathbf{u} / \partial t|}{\left|\eta \nabla^{2} \mathbf{u}\right|}
$$

The flow around the obstacle can be described by the Stokes equations if $S t<<1$. Supposing that the strain causing fluid accelerations derives from momentum diffused away from neighbouring particles also embedded in the fluid, the variable $t$ takes on the meaning of the momentum diffusion time scale, and implies that momentum transport by viscous diffusion

\footnotetext{
${ }^{3}$ Since the observation of this effect predates the Navier-Stokes law.
} 
must occur on a time scale $t>>d_{p}^{2} / \eta$, in order for it to be true that neighboring particles should not experience accelerations of the carrier phase due to one another. In Lambrechts et al. (2016), we evoked a similar criterion in order to justify modeling the disperse phase as a fluid, assuming that due to the large interparticle separations, $\phi^{-3 / 2}$, momentum diffusion would not contribute to the velocity dispersion, $\sigma$ of the particles.

While quite a lot is known about particle suspensions, there is a striking difference between the previously studied systems and that relevant to a PPD, mainly arising from the necessarily large value $e^{4}$ of $\Delta \rho$; for the particles to be immune to Brownian motions of the gas, while the drag law simultaneously approaches free-molecular flow conditions, the gas pressure and density must be lower than atmospheric conditions. Sedimenting particle suspensions at pressures approaching the Epstein regime have not been well-studied in the laboratory or otherwise.

Consider the consequences of the dilute carrier phase. First of all, solid particles are hardly buoyant, requiring a fast counter flow to suspend them, as in a fluidized bed. At the same time, low gas density conveniently lowers the container- and particle-scale $R e$, satisfying the requirement of a laminar flow. Secondly, the large mismatch in $\rho_{g a s}$ and $\rho_{p}$ in a mixture with $\varepsilon$ of order unity leads to an extremely low $\phi$ and large mean inter-particle separations. Therefore, well-known recipes from sedimentation vessels, such as equations 2.2 and 2.1 that depend mainly on $\phi$, are difficult to apply. Still, large $\phi^{-3 / 2}$ is useful for comparison with a PPD because the criterion set by equation 2.3 is met. Thirdly, the disparity between $\rho_{\text {gas }}$ and $\rho_{p}$ will result in a significant slip velocity between the disperse and carrier phases. This feature may strengthen the comparison to the dusty-fluid model, since the SI arises due to differential motion of the gas and dust phases.

The large $\phi^{-3 / 2}$ has practical consequences for the experimentalist as well. For example,

${ }^{4}$ The reader can get a feel for this by noticing that solid material densities are in the range $1-8 \mathrm{gm} \mathrm{cm}^{-3}$, whereas the density of air at atmospheric pressure is $0.001 \mathrm{~g} \mathrm{~cm}^{-3}$, and obviously decreases for lower pressures. 
finite volume effects become dominant as the particle separation approaches the size of the measurement window. Furthermore, the diluteness of the disperse phase precludes using a standard technique such as particle image velocimetry (PIV) to measure the particle velocities. In performing PIV, one calculates the velocity fields by correlating the positions of particles in subsequent pairs of images. The reliability of the derived velocities depends upon being able to match sufficiently large number of particles (usually at least 10) inside of a fixed interrogation window. To study clustering on small spatial scales, the interrogation window should be small. Alas, for a dilutely seeded flow, where one seeks to identify inhomogeneities in the background value of $n$, PIV will clearly fail.

Thankfully, the problem of studying fluid flows with intermittent local particle concentrations and velocities, both in their Eulerian and Lagrangian frames, has already been met for other types of complex flows. Namely, in studies of turbulence. Complex fluid motions can be traced by tracking individual, tightly coupled (low $T_{f}$, neutrally buoyant ), particles using high speed cameras. Using a consistent spatial reference guide for multiple cameras yields three-dimensional particle positions in time, from which velocity and acceleration statistics can be derived (La Porta et al. 2000, Ouellette et al. 2006, Xu et al. 2006, 2007, Xu 2008). In addition to studying the flow itself using tracer particles, the response of inertial particles, posessing a wide range of $S t$, to turbulent motions can be studied using comparable methods (Bourgoin 2006, Xu \& Bodenschatz 2008, Gibert et al. 2010, Saw et al. 2012, 2014).

\subsection{A novel facility}

A version of the following was published in Astronomy and Astrophysics (A\&A), Volume 591, Article number A133, Year 2016, as 'Spontaneous concentrations of solids through two-way drag forces between gas and sedimenting particles', by M. Lambrecths, A. Johansen, H. L. Capelo, J. Blum, and E. Bodenshatz. 
We conducted a numerical study to support ongoing work to investigate drag instabilities in the laboratory. A full description of the apparatus constructed at the Max Planck Institute for Dynamics and Self-Organisation and first results will be presented in an accompanying paper (Capelo et al, in prep). Here, we restrict ourselves to highlight some aspects relevant to the understanding of the numerical results to be presented in [Ch 5 of this thesis].

The experimental apparatus consists of a cylindrical vessel, housing a gas stream operating at pressures in the range of $0.5-10^{3}$ millibar. The axial component of the cylindrical flow is parallel with the direction of Earth's gravity, similar to the sedimentation configuration in the simulations. The upwards steady-state flow is seeded with weakly inertial particles, with typical sizes of $20-90 \mu \mathrm{m}$. The range of operational pressures and temperatures, listed in Table 2.1, then allows us to span both the Stokes and Epstein drag regimes.

The particle entrainment happens upstream in the fluid flow. There the system is in a brief transient state. The solids are transported and mixed with the gas by the time the flow reaches the steady-state conditions in which the measurements are to be made. This is done to make a fair comparison with the nearly homogeneously mixed initial conditions of the two-fluid dust/gas models.

Table 2.1 summarises the parameter region in which the experiment operates, including gas state variables, Mach and Reynolds numbers. The flow conditions are incompressible and laminar. The experiment is first in its kind probing the Epstein drag regime in a fluid with equal mass loading of gas and particles.

The experiment described here is somewhat analogous to particle suspension experiments in Newtonian fluids with low particle Reynolds number (Guazzelli \& Hinchdoychev 2011). However, in those studies volume fractions, $\phi=n_{\mathrm{p}} R^{3}$ (with $n_{\mathrm{p}}$ and $R$ the particle number density and radius, respectively), are no lower than $\phi \approx 0.01 \%$. Our experiment operates at $\phi \approx 10^{-4} \%$, when the dust-to-gas ratio is unity, for solid spherical particles with densities ranging from that of vitreous carbon $\left(\rho_{\bullet}=1.4 \mathrm{~g} \mathrm{~cm}^{-3}\right)$ to steel $\left(\rho_{\bullet}=8 \mathrm{~g} \mathrm{~cm}^{-3}\right)$. The low particle 
Reynolds number, $R e_{\mathrm{p}}$, in such suspension experiments comes from the use of a fluid with high dynamic viscosity. The particles are very buoyant and slowly creep through a thick liquid. Here, on the other hand, the low values of $R e_{\mathrm{p}}$ come from the fact that the kinematic viscosity becomes high when the gas density is low. It is encouraging that such experiments, even if in a regime different from the one studied here, show interesting particle dynamics (Batchelor 1972), such as particle Rayleigh-Taylor mushrooms and drafting particle trains (Pignatel et al. 2009, Matas et al. 2004). 
Table 2.1.: Parameters of the laboratory experiment. The range of pressure values correspond to different settings used to seed particles of various sizes and densities in the flow. The range in temperature values corresponds to the cooling that occurs as the gas expands to reach steady low-pressure conditions. The Reynolds numbers are calculated using the definition, $R e=\rho v L / \mu$, where $\rho$ is the density of the gas, $v$ the characteristic velocity, $L$ the characteristic length scale, and $\mu_{\text {air }}=1.8 \times 10^{-5} \mathrm{~kg} \mathrm{~m}^{-1} \mathrm{~s}^{-1}$ is the dynamic viscosity of air at room temperature. For the particle Reynolds number, we take the characteristic velocity and size to be the terminal velocity and the particle diameters, respectively. The density of the gas is estimated using the measured values of temperature and pressure, assuming a mean molar mass of air $M_{\text {air }}=0.02891 \mathrm{~kg} \mathrm{~mol}^{-1}$ and molar gas constant $R=8.314 \mathrm{~m}^{3} \mathrm{~Pa} \mathrm{~K}^{-1} \mathrm{~mol}^{-1}$. The global Reynolds number comes from the mean flow velocity and the tube diameter. Similarly, the Mach number is the ratio of the mean flow velocity to the sound speed at the measured temperature, again assuming the same values of $R$ and $M_{\text {air }}$.

\begin{tabular}{ll}
\hline \hline Property & Value \\
\hline Working gas & air \\
Tube height & $1.6 \mathrm{~m}$ \\
Tube diameter & $9 \mathrm{~cm}$ \\
Friction time & $0.05-0.08 \mathrm{~s}$ \\
Friction length & $\approx 3-7 \mathrm{~cm}$ \\
Pressure range & $10-8000 \mathrm{~Pa}$ \\
Temperature & $16-22^{\circ} \mathrm{C}$ \\
Estimated mean flow speed & $1.2 \mathrm{~m} \mathrm{~s}^{-1}$ \\
Global Reynolds number & $0.6-6$ \\
Particle Reynolds number & $0.009-0.08$ \\
Mach number & 0.003 \\
Solid-to-gas ratio & $0.1-10$ \\
\hline
\end{tabular}


Time-resolved data on the particle trajectories is obtained from high-resolution cameras and 3-dimensional Lagrangian particle tracking (Xu 2008, Ouellette et al. 2006). This is a common technique to study both tracer and inertial particles in fluids. The typical measured and derived quantities are the probability density distributions of the particle velocities and accelerations, their statistical moments, and correlation and structure functions.

The obtained data do provide an interesting comparison to the results shown in [chapter 5 of this thesis]. The parameter regime is sufficiently similar to the numerical experiments that we expect the drafting instability to manifest itself. Particle tracking would not only allow the detection of particle swarms, but also the individual particle dynamics. For instance, [we demonstrate] that the growing maximum in particle velocity dispersion traces the increase in maximum particle density. Such statistical measurements of the particles will allow qualitative comparison between the numerical work and the experiments. 


\section{CHAPTER 3 \\ Experimental Methods}

This chapter is broadly divided into two halves: Section 3.1 is concerned with the aspects of the system related to controlling or measuring the gas properties, including the values of $P$, $T$, and radial velocity profile; section 3.2 is regarding the disperse particle phase, such as particle seeding, containment and tracking. In each subsection of this chapter I describe the design principles of the apparatus or employed technique and I follow up with a report on the system's performance.

The apparatus is a pipe-like vessel containing a particle suspension in which a low-pressure gas flow counteracts particle-sedimentation. The gas, seeded with inertial particles, is driven vertically by a global pressure gradient $\nabla P \approx 10^{4} \mathrm{~Pa}$. I define vertical as the direction opposing gravity, $z$, with $x$ and $y$ being the cross-stream directions perpendicular to the $z$-axis. Figure 3.1 shows a photograph of the facility and figure 3.2 shows a rendered mechanical drawing of the apparatus. The direction of positive $z$ is 'up' in these images. 


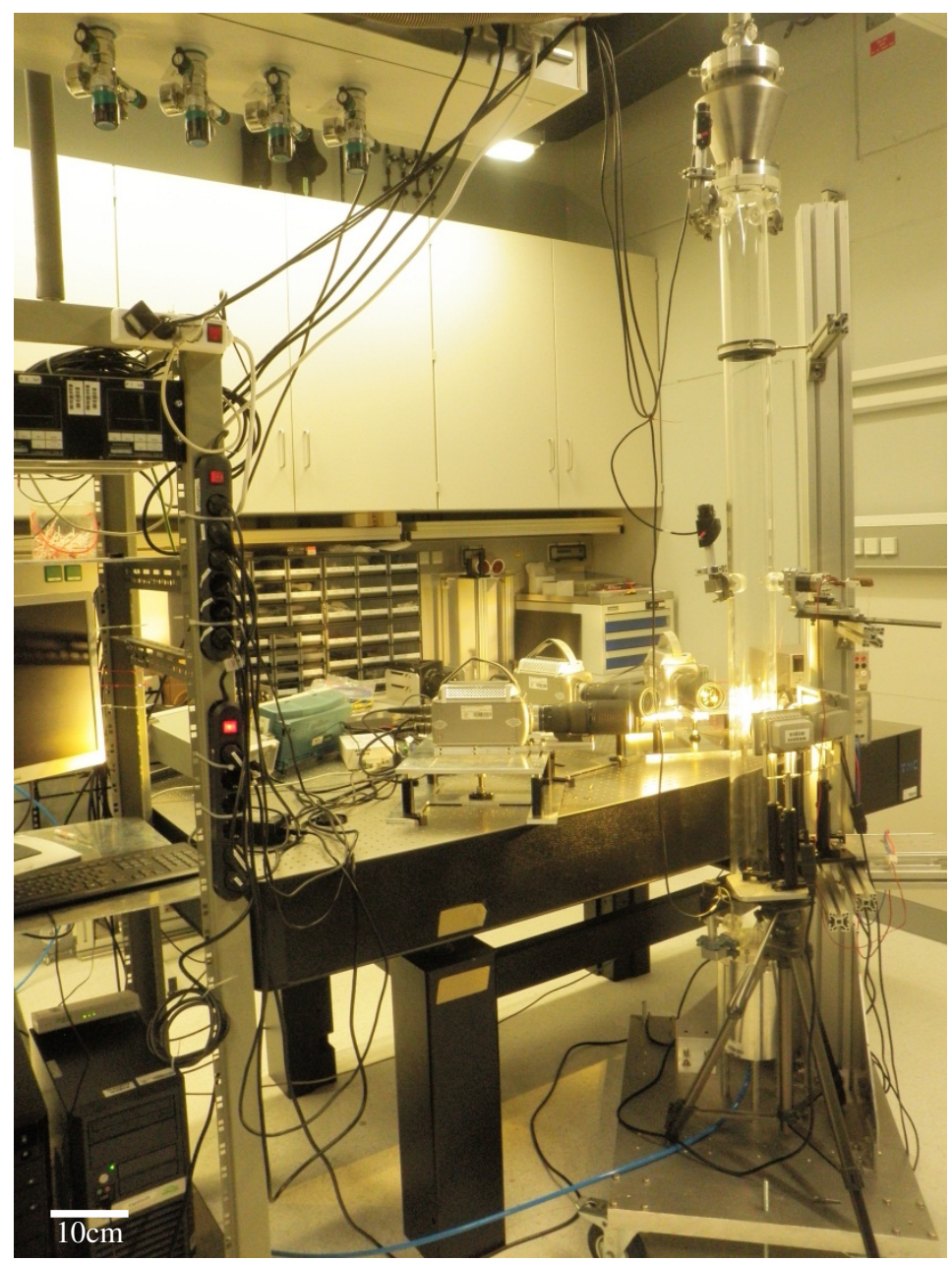

Figure 3.1.: Image of the experimental facility. The cameras and lighting are configured for Lagrangian Particle Tracking. 


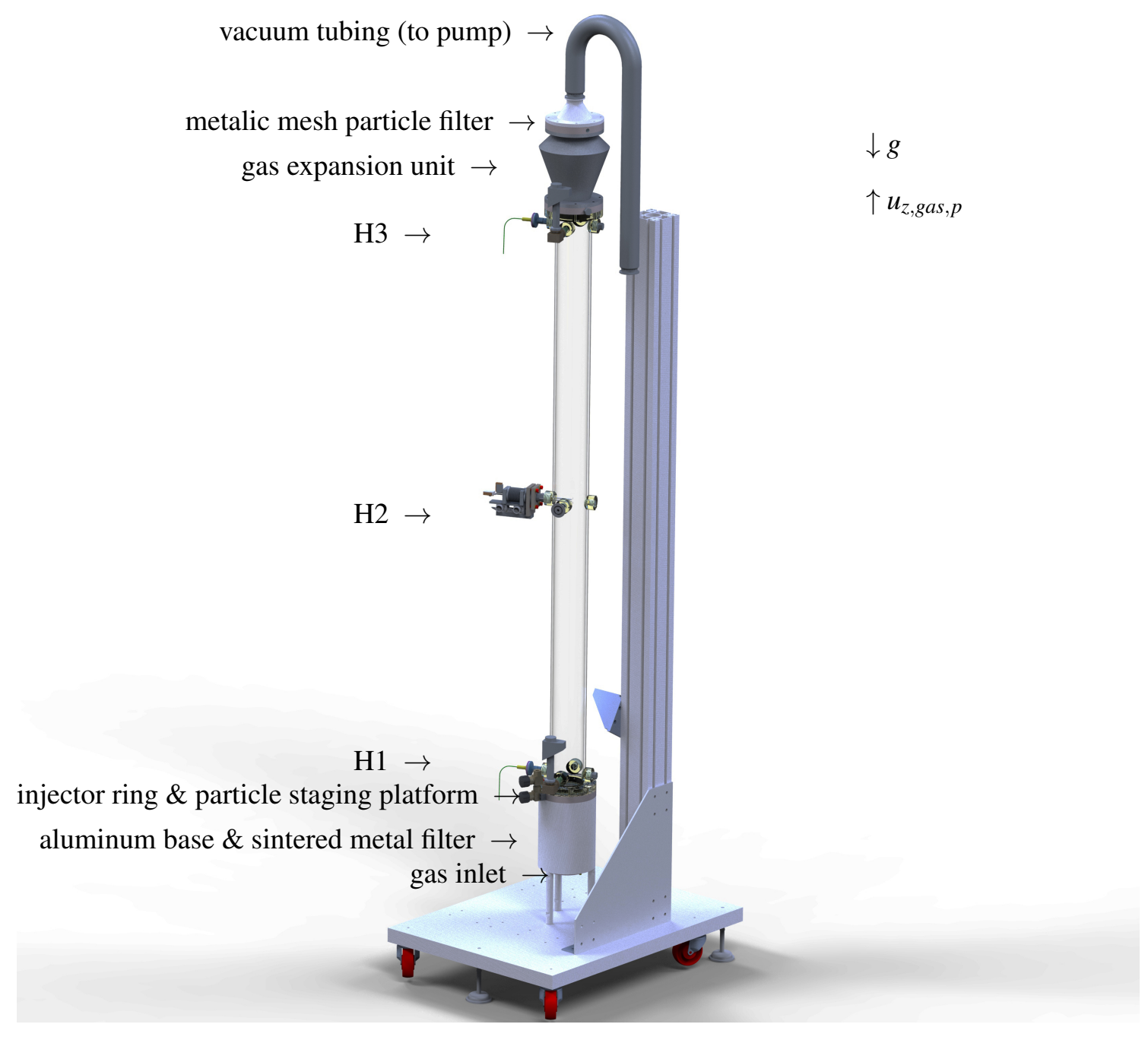

Figure 3.2.: The unit sits on a tripod and gas enters from the underside. Inside the aluminum base of the apparatus is a pressure-reducing filter and at the top is a hollow expansion unit. Vacuum tubing leads from the expansion to a rotary-vane vacuum pump with adsorption trap (of activated aluminum oxide). The small caps along the side of the tube are threaded entry points where measurement devices (pressure transducers, thermocouples and their feedthroughs) are secured; there are four on the bottom (H1) and top of the tube (H3) and two in the center $(\mathrm{H} 2)$. The exeriments in this thesis were conducted with the camera viewing height just below $\mathrm{H} 2$. The components connected by flanges are sealed with o-rings. The threaded attachment points are cut at tapered angles and are wrapped in teflon. The column to the right of the tube is an alumnium optical profile, used in various ways from securing the tube with braces (not shown) and to mounting additional hardware, lighting, or optical elements. The direction of particle and gas motion is indicated. 
Due to the geometry of the system, I will often assume cylindrical symmetry in discussing the mean flow profile or the coordinate locations of the particles, and define $r=\sqrt{x^{2}+y^{2}}$, where the maximum radius is half the tube diameter, $\mathrm{D}_{t} \sim 9 \mathrm{~cm}$. The properties of the gas carrier phase are studied for the global system: I measure time-resolved state variables and calibrate the mean flow-profile for the central $\sim 6 \mathrm{~cm}$ of the tube using two-dimensional PIV described in section 3.1.4 ${ }^{1}$. The properties of the disperse particle phase are measured in a much smaller volume, of order $\sim 1 \mathrm{~cm}^{3}$. I use a three-dimensional Lagrangian Particle Tracking system (LPT; described in section 3.2) to extract the positions, velocity, and accelerations of individual particles, in order to carefully study the particle dynamics statistically.

Although the facility is equipped to transport several different species of gas to the apparatus, all of the experiments described here involve dry air as the carrier phase of the flow and solid steel particles as the disperse phase. These particles are suitable because their inertial forces far outweigh the electrostatic forces on their surfaces, therefore potential particle clustering could not be due to the latter ${ }^{2}$ (Okuzumi 2009). Furthermore, their terminal velocities are well matched to the flow speed of the system for sizes approaching particle-scale $K n \sim 1$.

\subsection{Flow Carrier Phase}

There are a variety of measurement techniques that rely primarily upon the fact that the resistance of a wire is altered in a predictable way when a temperature difference is applied across its length. Thermocouples, used to measure temperature, are nothing more than two dissimilar wires of known electrical resistivity, point welded together at one end (the sensor) and connected to positive and negative terminals at the other. When a temperature change is

\footnotetext{
${ }^{1}$ Although this is a particle-based technique, these calibration measurements of the carrier phase, for which I used fluidized aerosols as tracers, should not be confused with the measurements of the inertial steel particles.

${ }^{2}$ And for practical purposes, they would not stick to the polycarbonate walls of the container.
} 
applied to the sensor, a voltage difference results across the terminals. Provided that there is a reference temperature measurement at one end of the wire, the temperature at the location of the sensor is known directly from the measured voltage drop.

More involved devices build upon the temperature-dependent resistivity of a wire, but rather continuously supply a current to keep the resistance constant using an electrical bridge. Measuring the supplied power in the presence of temperature variations yields direct information on the state of the gas. In particular, the gas' thermal conductivity decreases directly with decreasing gas density, whereas its thermal diffusivity increases: $D_{t h}=k / c_{p} \rho_{g a s}, k$ being the conductivity, $D$ the diffusivity, and $c_{p}$ the specific heat capacity. Pirani pressure gauges apply this principle directly to obtain the pressure of an ideal gas.

I integrated both of the aforementioned measurement devices into the apparatus, which is discussed further in this section. Figure 3.3 shows a photograph of the devices connected to the apparatus. 


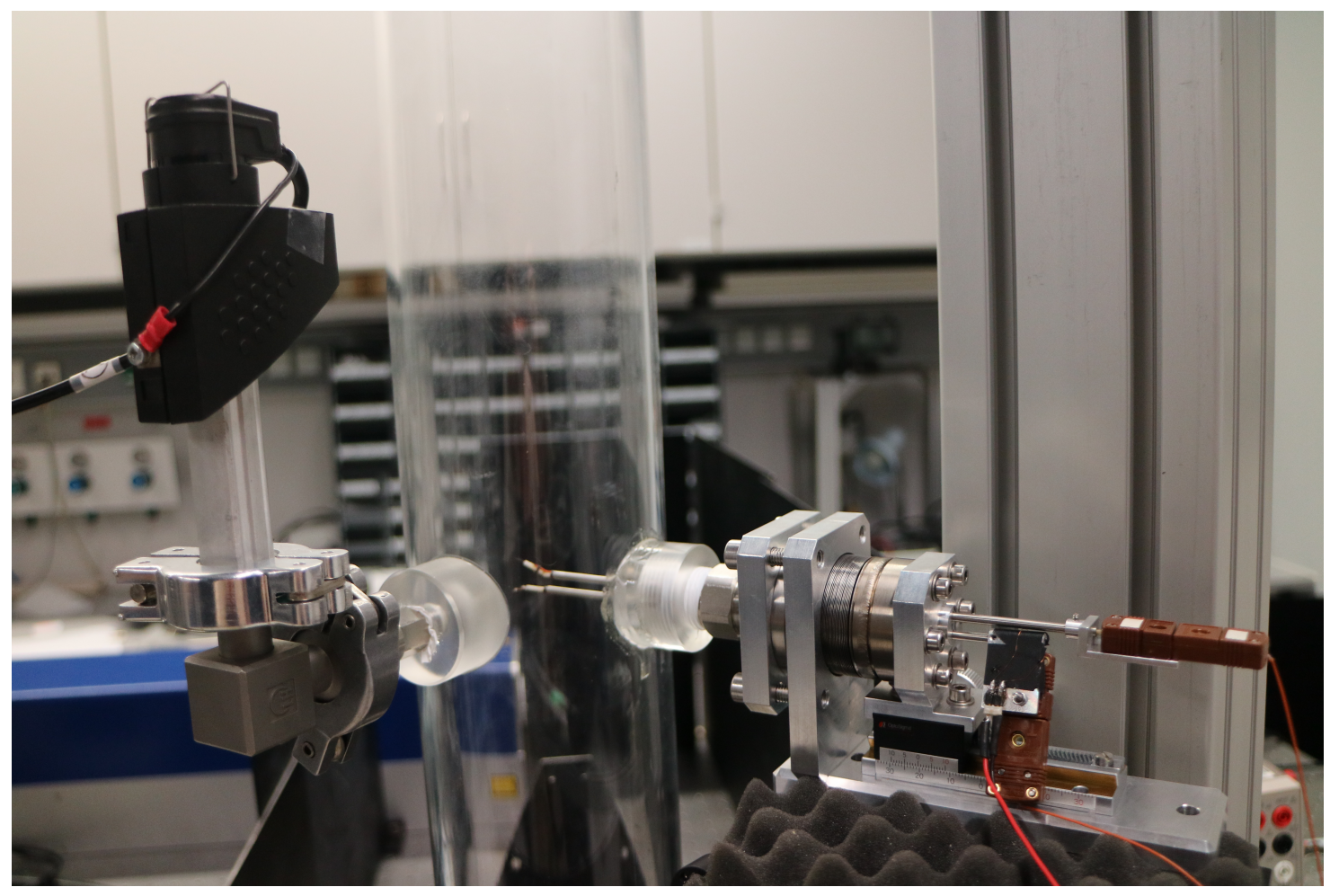

Figure 3.3.: A Pirani pressure head (left) and thermocouple vacuum feedthrough (right) attached at the point labeled $\mathrm{H} 2$ in figure 3.2. There is a $3 \mathrm{~mm}$-diameter hole in the wall of the tube to let the rarefied gas reach the pressure head and there is a $3 \mathrm{~mm} \times 8 \mathrm{~mm}$ vertical slit cut into the wall to let pass the temperature probe. 


\subsubsection{Pressure Control and Measurement}

\subsubsection{Creating a Pressure Drop}

The target range of the pressure is set by the wish to simultaneously study collective particle dynamics while (1) $K n$ with respect to the particle size is large, and (2) $R e$, when calculated using either the container diameter or $d_{p}$ as the characteristic length, is small . One must achieve vacuum conditions to increase $\lambda$, but since lower $\rho_{\text {gas }}$ requires a correspondingly low particle number density to keep $\varepsilon$ in the desired range, this effectively puts a floor on the strength of the vacuum under which the experiments should be conducted. The measurement volume is ultimately limited by the particle tracking technique, which is explained in detail in the next section.

I achieve low-pressure conditions by packing the flow-chamber gas inlet with a porous material ("porous filter") possessing high flow impedance. I apply a vacuum to the other end of the chamber, and the pressure differential across the whole system drives the flow. Figure 3.4 shows the cross-sectional view of the apparatus base. The external aluminum cylinder holds a cone comprised of stacked sintered-metal plates. The plates were precision machined so that their surfaces are smooth and in tight contact and similarly, the outer edge of the cone is continuous from one plate to the next. The purpose of the conical geometry is to reduce the net force on the high-pressure end of the filter. The two components were assembled by first isotropically expanding the aluminum base by heating it, then dropping the filter in and allowing the external holder to cool and contract around the porous filter. 

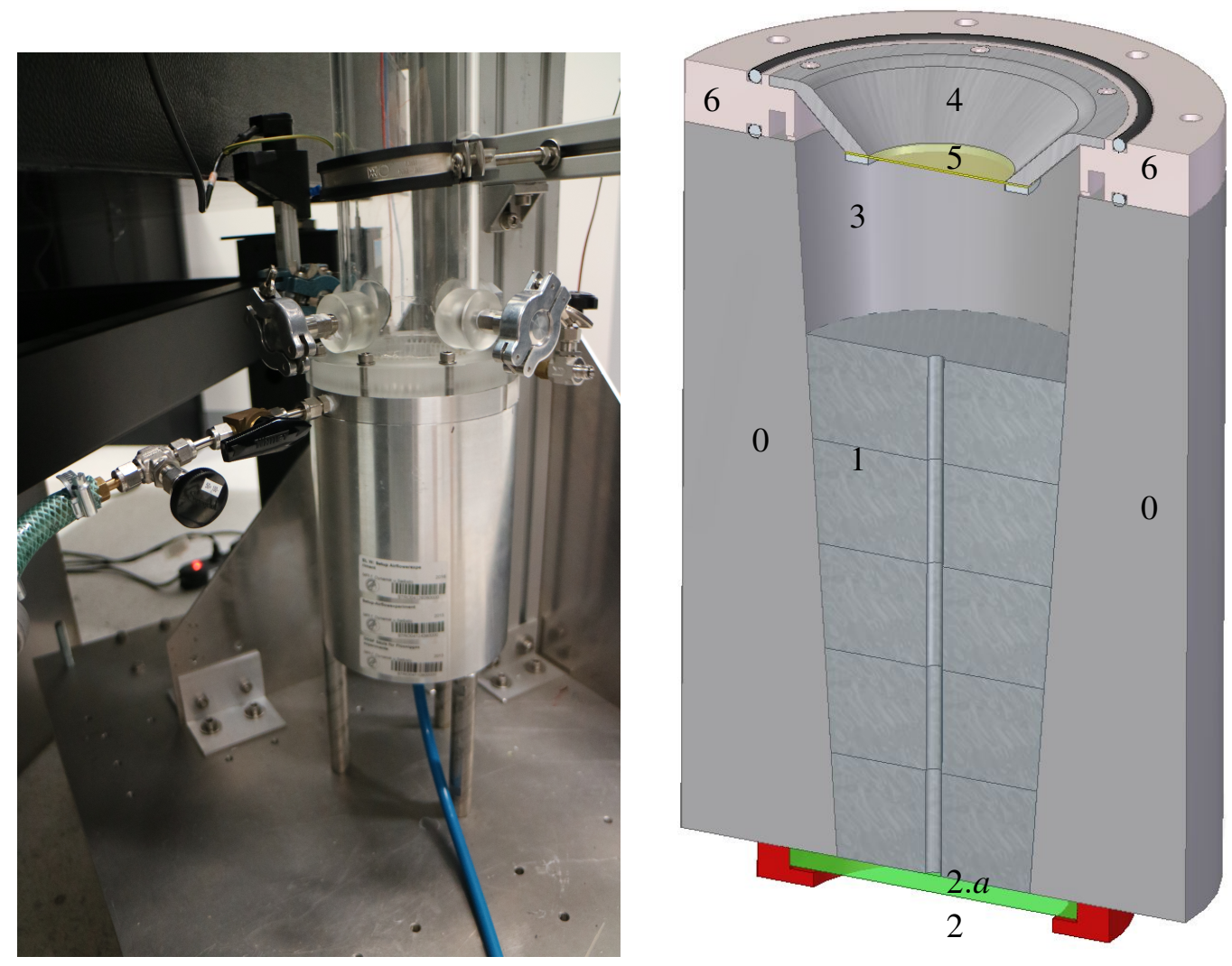

Figure 3.4.: Photograph (left) and cut-away view of the apparatus base. (0) The external holder is solid aluminum. (1) The interior is a cone of stacked sintered-metal cylinders of porosity coefficient $\chi$. (2) The air inlet is at the bottom. Shown is a dust filter (2.a), although there is also the option to attach an adaptor that delivers pressurized gas to the inlet (see blue tubing in photograph). (3) The hollow chamber provides the option to add additional flow-impeding material. $(4,5)$ The tray installed above the chamber is the particle-seeding bed; (4) The tapered part of the tray is made of aluminum and (5) the flat bottom is a fine wire mesh through which air can pass, but solid particles cannot. (6) Also shown is the perforated ring, through which gas can be injected directly into the system, without passing through the metallic filter (see valve assembly in photograph and details in figure 3.5). 
To design the porous filter, I estimated the height, $h$, of porous material required to reach the desired operational pressure in the tube by considering the balance of momentum mediated over the opening area of a representative pore in the medium, which is characterised by a porosity coefficient, $\chi$. The expression for the momentum balance is simply

$$
\frac{d P}{d h}=-\frac{\mu}{\chi} u(h)
$$

where mass conservation implies, assuming an ideal gas,

$$
u(h) P(h) a(h)=u_{t} P_{t} a_{t} .
$$

Hence:

$$
P(h)=-\frac{\mu u_{t} a_{t} P_{t}}{\chi} \int_{P_{t}}^{P_{\text {inlet }}} \frac{1}{a(h)} d h .
$$

The coefficient depends upon the desired conditions in the tube which are indicated with subscript $t$. The target parameter values I used were pressure $P_{t}=1 \mathrm{mbar}$, area $a_{t}=a(d=$ $9 \mathrm{~cm}$ ), and flow velocity $u_{t}=1 \mathrm{~m} \mathrm{~s}^{-1} . \mu$ is the viscosity of air, $1.5 \times 10^{-5} \mathrm{~Pa} \mathrm{~s} . h(0)$ is inside the flow chamber and $h$ takes increasingly negative values towards the air inlet, where the pressure is atmospheric, i.e. $P_{\text {inlet }}=1$ Bar. Note by inspection that the lower the value of $\chi$, the shorter the distance over which the pressure drop is achieved. For the material I used, $\chi=0.08 \times 10^{-12} \mathrm{~m}^{2}$, the value of which was determined by the product manufacturer using Darcy's law. If the shape of the sintered filter were cylindrical, then $a(h)$ would be constant and equal to $a_{t}$, but for the conical shape with opening angle $\beta$, the area varies with height as $a(h)=\pi(r-h \times \tan \beta)^{2}$, where $r=D_{t} / 2$. The integrand is:

$$
P(h)=\left\{P_{t}^{2}-\frac{2 \mu P_{t} u_{t} a_{t}}{\chi \pi}\left[\frac{1}{(\tan \beta)(r-h \times \tan (\beta))}-\frac{1}{(r \times \tan (\beta))}\right]\right\}^{\frac{1}{2}} .
$$

The height of the filter is estimated as the value of $h$ which returns the inlet pressure of 1 bar, 


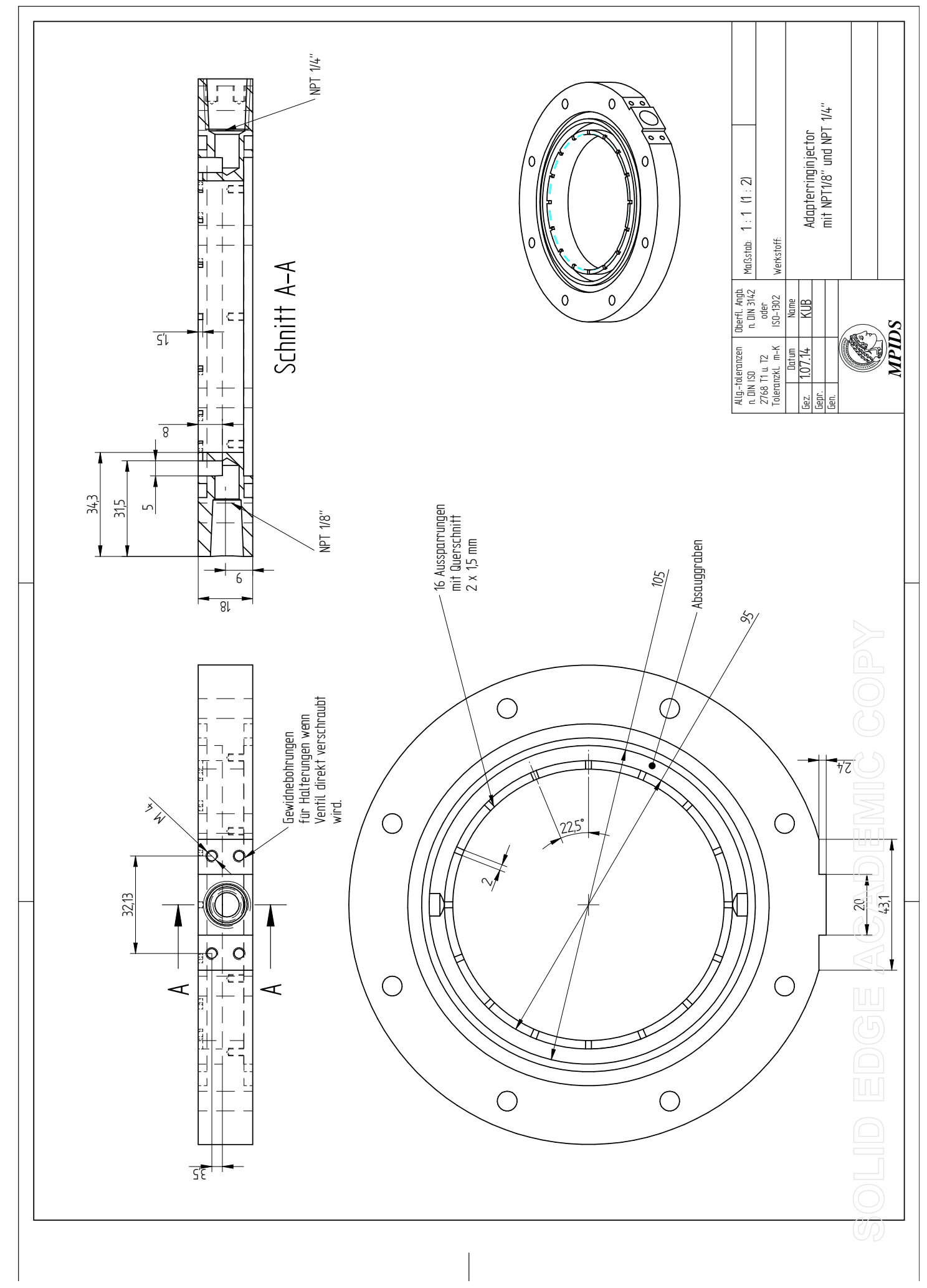

Figure 3.5.: mechanical drawing of the perforated ring, prepared using Solid Edge cad software, by Artur Kubitzek. Gas valves are connected via one of the threded entry points labeled "NPT 1/8" or "NPT1/4". Through one of these entry points (the other remains sealed), gas enters into and fills the channel (labeled 'Absauggraben') and passes through the 16 rectangular perforations (labeled ' 16 Aussparrungen mit Querschnitt $2 \mathrm{x}$ $1,5 \mathrm{~mm}$ ') into the hollow chamber below the particle seeding platform (see figure 3.4). 
and depends only upon $\beta$, for a fixed $\chi$. Because this expression is algebraically cumbersome, I optimized the dimensions iteratively, by supplying trial values of $\mathrm{h}$ in increments of three $\mathrm{cm}^{3}$. I also optimised the opening angle by considering the net force on a given layer of material in the filter ${ }^{4}$. I found that an opening angle $\beta=4^{\circ}$ and height $15 \pm 1 \mathrm{~cm}$ would produce the requisite pressure drop.

\section{Time-resolved Pressure Log}

I measure the pressure at three locations in the tube, once each at the bottom, middle and top (labeled H1, H2, and H3 in figure 3.2), using Thermovac Pirani pressure heads (pictured in figure 3.3) with tungsten filament, suitable for Pressures in $0.5 \times 10^{-3}-10^{3}$ mbar range. The circuitry from the pressure heads run to a COMBIVAC CM 31 pressure gauge. Communication between the pressure gaugue and the computer is over RS232, using a serial-to-ethernet adapter. The RS232 interface has nine pins, with the option to operate in either printer or remote control mode. I use the latter because the printer mode is fixed to send pressure readings every 10 seconds, whereas a desired frequency can be specificed for the remote mode, which uses three pins [transmitting line (TxD, pin 2), receiving line ( $\mathrm{RxD}$, pin3), and ground(GND,pin5)]. The device protocol is the American Standard Code for Information Interchange (ASCII code). The software wrapper to manage the communication is in the $\mathrm{C}++$ language ${ }^{5}$. I use the internal system time of the computer to send a measurement request twice every second to each of the three measurement channels, each corresponding to one of the pressure heads.

\footnotetext{
${ }^{3}$ The thickness of the cylindrical bricks I used.

${ }^{4}$ Since $F_{n e t}(h)=\frac{d P(h) a(h)}{d h}=P(h) \frac{d a(h)}{d h}+a(h) \frac{d P(h)}{d h}$, the area of the conical filter cannot decrease too rapidly, or else the sign of the net force will change, representing a breakdown of the assumption that the horizontal expansion is negligible by comparison to the $z$-direction gas discharge rate.

${ }^{5}$ This software was written as part of a three-month student project by a visitng intern from ENS Lyon.
} 


\section{Pressure Performance}

In practice, the sintered-metal filter produces the requisite pressure drop. I have the freedom to make arbitrary adjustments to $P_{t} \sim 1 \mathrm{mbar}$ by either over-pressurizing the gas at $P_{\text {inlet }}$ or by injecting miniscule amounts of gas directly into the top of the apparatus base via a needle valve that passes through a perforated ring to enter the chamber on all sides. Figure 3.6 shows a measurement of the pressure history when the system is operating. Starting from the initial atmospheric pressure, I evacuate the system continuously until it reaches a constant steady-state pressure (SS1). The small bump in the pressure corresponds to when the needle valve was opened. After a brief transient phase, labled 'equilibration' in the figure, the measured value of the pressure approaches a new limiting steady-state pressure (SS2). Note that this is just an example, SS1 and SS2 can be varied arbitrarily; SS1 is varied (in the range $\sim 0.5-5 \mathrm{mbar}$ ) by changing the pressure at the gas inlet and SS2 is adjusted (to values between 1 and 1000 mbar) via the needle valve. Although making adjustments to SS2 involves injecting atmospheric pressure gas, it equilibrates with the low-pressure gas at the sound speed, which is two orders of magnitude larger than the typical flow velocity of the system, as will be demonstrated in the next section.

The needle valve and perforated ring fixture shown in figures 3.4 and 3.5 play a role in particle seeding, both for the PIV and LPT measurements. The smoke used in the flow calibration passes directly through the holes in the perforated ring. The inertial particles are pre-seeded inside the chamber and I use the density-dependence of the particle drag force to select the value of SS2 that will fluidize them. To avoid confusion about the two types of particles used in different sets of experiments, I will refer to the process of introducing smoke into the apparatus (discussed in section 3.1.4) and to the smoke itself as seeding material; I will refer to the introduction of the steel inertial particles (discussed in section 3.2.1) as particle staging and will refer to the particles themselves as the disperse phase. 


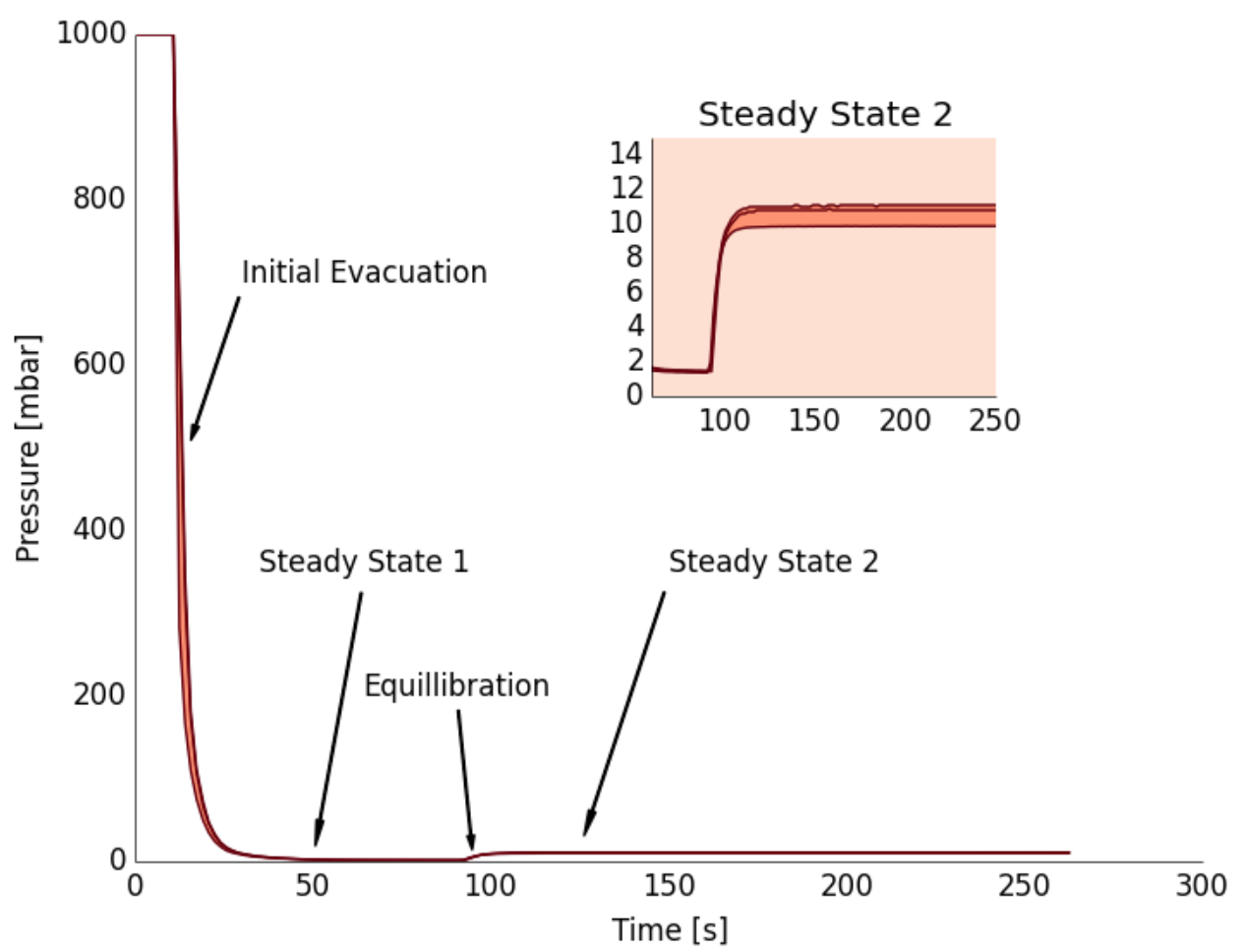

Figure 3.6.: Time-resolved pressure measurements for three Pirani pressure heads, placed at the top, middle, and bottom of the apparatus $(\mathrm{H} 3, \mathrm{H} 2, \mathrm{H} 1)$. The thickness of the line is due to the offset in the three pressure heads. Particles are seeded at the equilibration phase and remain entrained throughout SS2. The insert shows the jump in pressure (equillibration, resulting in SS2) from 1.5 mbar to 10 mbar. 


\subsubsection{Temperature Measurement}

\section{Equipment}

I made no special attempt to control the temperature, except that the climate control system in the laboratory holds the ambient conditions around $22{ }^{\circ} \mathrm{C}$. Using thermocouple (type $\mathrm{T}$, copper and constantan wires) temperature sensors, I read out the temperature through a Keithley 2701 Multimeter, operating in rear-panel mode, with a solid-state multiplexor switch. The reference junction temperature is measured internally, where the sensor lead attaches to the multiplexor. 


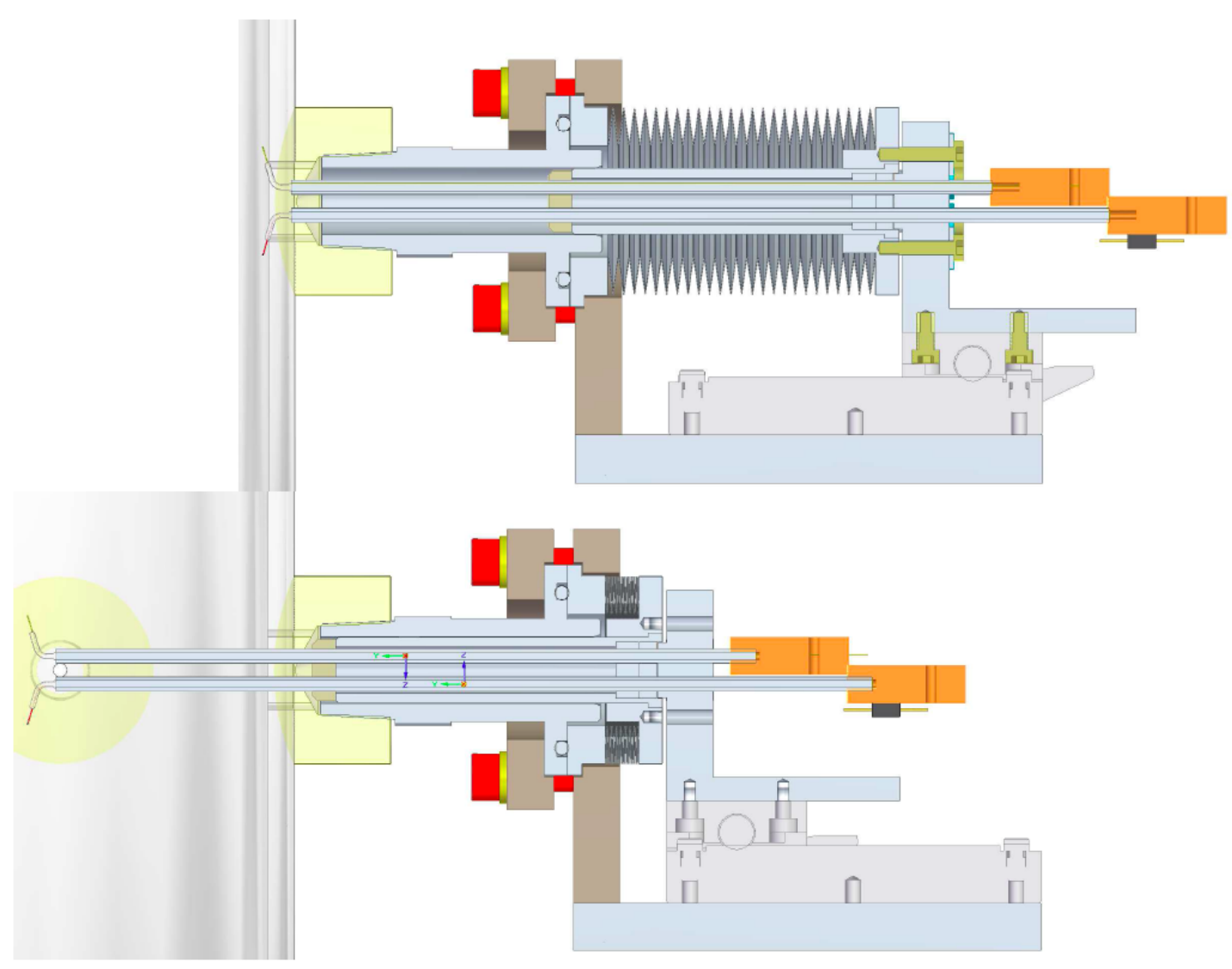

Figure 3.7.: Vacuum-sealed thermocouple feedthrough. 
The lead wires from the thermocouples must reach from inside the apparatus to the multimeter/multiplexor terminals. Since all contact points must be composed of the same material as the wires, the leads cannot be soldered to a standard electrical vacuum feedthrough. I therefore designed and built an assembly of ceramic insulation tubes that is encased in a bellows and sealed with a KH vacuum flange, shown in figure 3.7. feedthrough which houses ceramic insulation tubes, through which the thermocouple wires feed. The feedthrough slides on a linear stage, so that the sensors can probe the centerline as well as closer to the walls ${ }^{6}$.

\section{Time-resolved Temperature Logging}

I communicate with the multimeter over ethernet using an ethernet TCP/IP socket and sending commands in the Standard Commands for Programmable Instruments (SCPI) protocol. I wrote the wrapper software for this communication in the $\mathrm{C}$ language. The device buffer can hold up to 45,000 measurements, so I store the measurements on board the device and then send a request for them after the desired time period is over. Each measurement consists of the temperature value in degrees Kelvin, the internal time stamp of the measurement relative to the first measurement in the series, and the measurement number. The time integration constant of the measurement is set to cancel the internal system noise, resulting in a measurement frequency of $40 \mathrm{~Hz}$. If one wants to tolerate this noise, the frequency is $\sim 200 \mathrm{~Hz}$.

\section{Thermal Gas Expansion}

I made time-resolved temperature measurements in the apparatus to determine how the gas expands. I found that the temperature decreases only by a few degrees when the system is in

\footnotetext{
${ }^{6}$ This design is needlessly complicated for just measuring the temperature. However, In addition to calibrating the temperature of the gas, it was my intention to measure the flow velocity profile by calculating the delay time of a heated gas parcel traveling between two points in the stream. It was for this purpose that I assembled the feedthroughs so that two temperature sensors are separated by $1.5 \mathrm{~cm}$ and oriented along the $z$-axis. The lower ceramic tube has additional holes through which the leads to a heating wire pass. This flow meter was not ultimately used to calibrate the flow profile.
} 
steady-state condition, after a large temperature drop during the initial system evacuation. Accordingly, I adopt an operational value of $293 \mathrm{~K}$ for all calculations that rely on the state variables of the gas, including $\rho_{g}, v_{t h}$ and by extension $\varepsilon, M a$, and $F_{d}$ in the Epstein regime. Several of these derived parameters are listed in table 2.1 and their values are implicit in additional calculations.

\subsubsection{Gas Flow Velocity Profile}

The mean gas flow velocity in our experiment is given by the vacuum volumetric throughput and the cross-sectional area of our measurement section. For a pumping rate of $25 \mathrm{~m}^{3} \mathrm{~h}^{-1}$ (which remains constant over several decades in pressure) and a tube diameter of $0.089 \mathrm{~m}$, I expect an average gas flow velocity between 1 and $1.5 \mathrm{~m} \mathrm{~s}^{-1}$. Our setup is essentially a vertical pipe flow and therefore I considered a priori that there might be a Pouseille flow profile.

I calibrated the flow profile using PIV, with fluidized aerosols (smoke) as the seeding material. Although this is a robust method, I was limited at which pressure I could conduct the measurements, due to the challenge of injecting a sufficiently dense tracer particle seeding while simultaneously maintaining steady vacuum conditions ${ }^{7}$.

I conducted the PIV calibration measurements at 10 mbar and reasoned that the flow profile should not change much for lower pressures for the following reasons:

- The throughput of the vacuum pump is the same over a wide range of pressures, well below and above the target operational pressure of $\sim 1-10$ mbar.

\footnotetext{
${ }^{7}$ Measuring spatially-resolved fluid flow under low-pressure conditions poses unique challenges, which mostly have never been met before, because although vacuum chamber technology is standard in laboratory and industrial settings, flows in a moderate vacuum are not very commonly studied. Typical flow measurement instruments require a minimum operational pressure of one bar or greater and the impracticality of using them under vacuum conditions varies depending upon the technique. In Appendix 3 I illustrate how a proven method to measure flow velocity at high pressure was insufficient under vacuum conditions, primarily owing to the high thermal diffusivity and low momentum diffusivity.
} 
- In the target pressure range, the container-scale $R e$ changes by only a small amount, never exceeding $\sim 50$. In any and all cases, the flow should be laminar. The relatively high 10 mbar pressure is the 'worst case scenario', because it has the highest $R e$ with regard to the hydrodynamic stability of the fluid.

- The value of $K n$ on the container scale also changes by a relatively small amount in the pressure range from 1-10 mbar. In no case should the no-slip boundary conditions expected of viscous flow be relaxed at the container walls. An estimate of the wall velocity for various pressures is given below.

\section{PIV Imaging, Illumination, and Optics}

PIV is a technique to measure instantaneous flow velocity fields by cross-correlating tracerparticle positions in subsequent image pairs. I implemented a two-dimensional PIV setup by focusing a laser sheet through the central cross-section of the apparatus. The plane of the camera chip is parallel to the sheet. The optical path of the laser was diverted by placing a mirror at 45 degree incidence. The beam was collimated using a convex cylindrical lens and the sheet was expanded by a convex bi-focal lens. Figure 3.8 shows an top view of the optical path of the laser. 


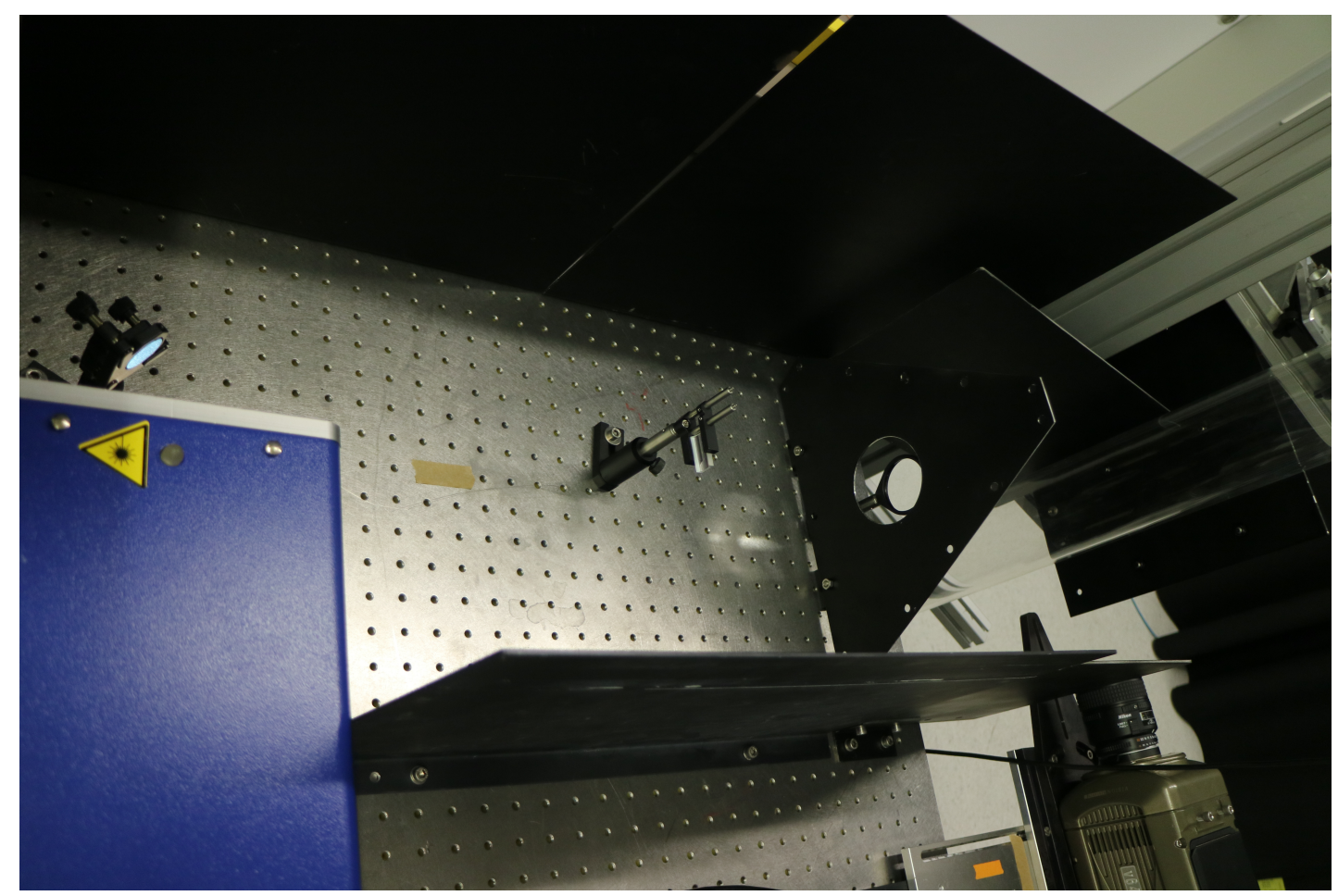

Figure 3.8.: Optical elements to collimate and expand the laser sheet for PIV measurements. The blue box is the laser housing and it is placed parallel to the camera, seen in the lower right of the image. The beam is diverted by a mirror placed at 45 degree incidence, then is colimated by a cylindrical lens and is finally expanded with a bi-focal lens. The black panels are beam blocks, which protect against stray laser light. The elements are aligned so the sheet passes through the center of the tube and is parallel to the camera chip. The calibration mask shown below was centered using the lasersheet as a guide. 
The calibration, using a mask with dot separation $2 \mathrm{~mm}$ and dot size $0.5 \mathrm{~mm}$, showed that the camera spatial resolution is approximately 25.6 pixels $/ \mathrm{mm}$. Figure 3.9 shows the fixture that holds the calibration mask. it is comprised of a circular plate with a linear translation stage attached to its underside. a a series of optical posts connects to the stage at one end and to a clear polycarbonate mask holder on the bottom. The mask, shown in figure 3.10, was printed on film, using the high-precision printing technique typically used to create photo-blocking masks in the creation of microfluidic devices. 


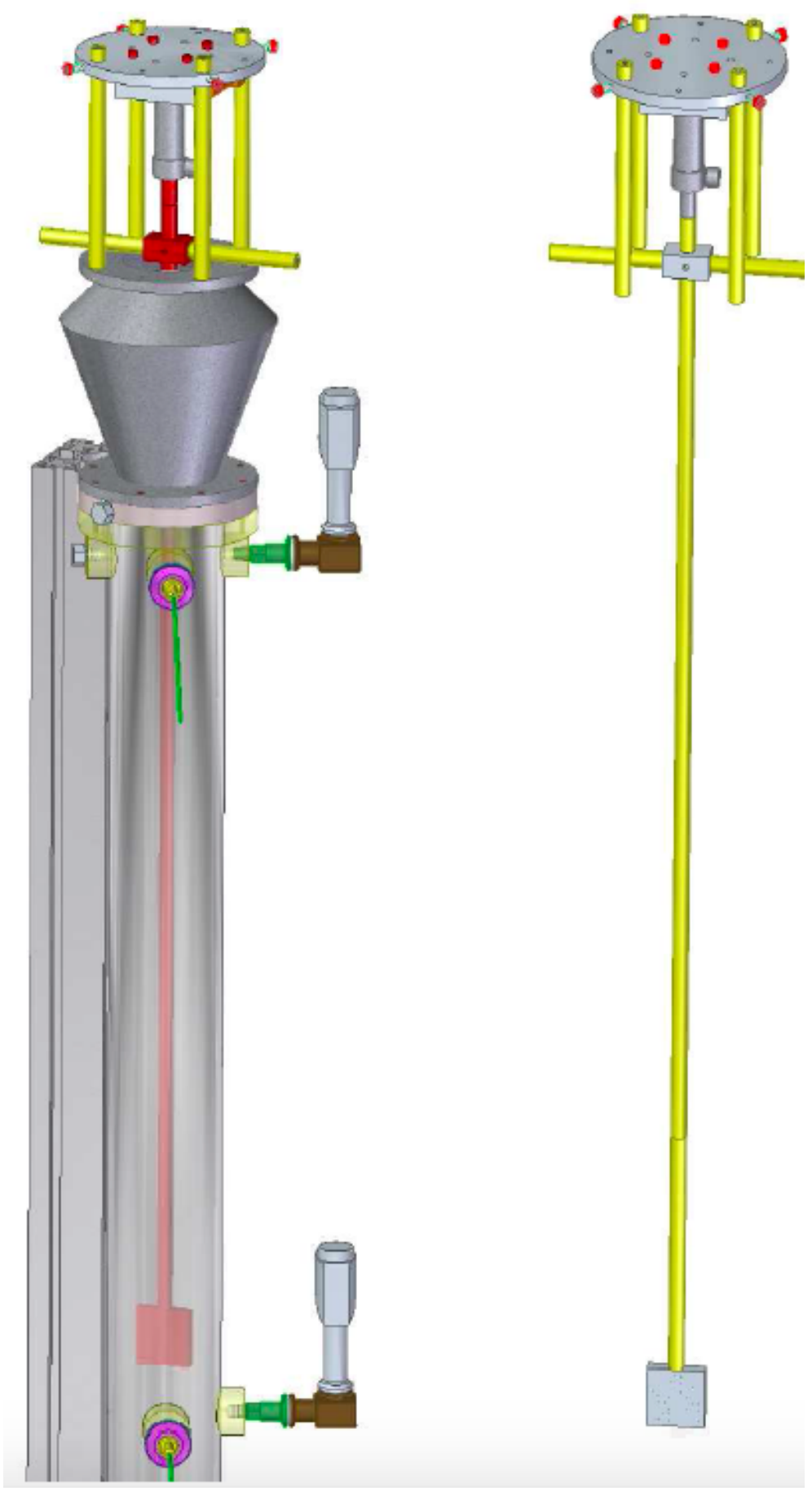

Figure 3.9.: Right: calibration mask mount. Left: mount inserted into the apparatus. The height of the mask can be adjusted by sliding the top plate along optical posts. There is a translation stage under the top plate so the mask can be translated in the $x-y$ plane. There is rotational freedom for mask adjustments also on the x-y plane. All calibrations are performed at ambient conditions. This mount was used for both the PIV and LPT measurement calibrations. In the former, the dot-pattern mask, shown in figure 3.10 was used and in the latter, the square-pattern mask, shown in figure 3.21, was used. 


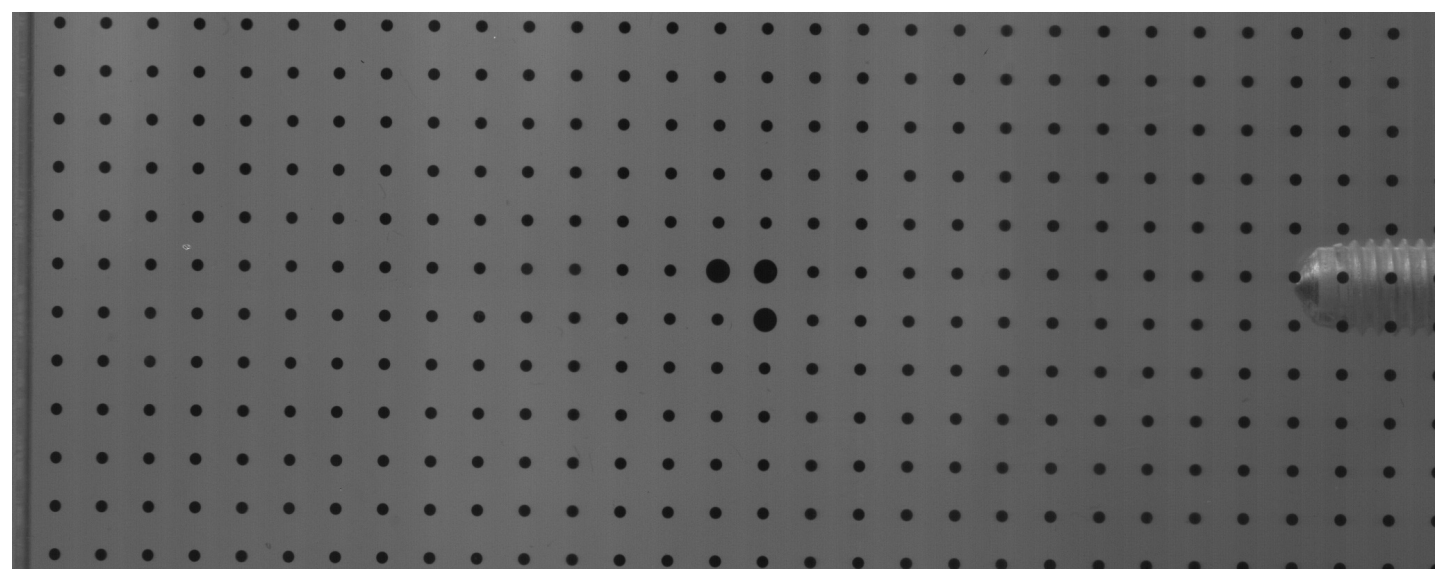

Figure 3.10.: Dot-pattern mask used to calibrate the spatial scale in PIV measurements.

Running in burst mode, using an interrogation time between subsequent frames $\Delta \mathrm{T}=$ $249 \mu \mathrm{s}$, the highest possible resolution of the v12 phanton camera is $2048 \times 1024$ pixels (as there is a trade off between sampling rate and resolution, limited by the camera's internal memory). The resultant field of view is a maximum $80 x 40 \mathrm{~mm}$. I reduced the width of the window to 60 millimeters to accommodate the calibration mask, which is necessarily smaller, since its fixture was designed to be able to traverse the tube.

I sampled with a time window between frame pairs of $450 \mu$ s and the interrogation window between the image pairs themselves involved $20 \mu$ s exposures every $300 \mu$ s. I pulsed the laser (IB Laser Chronos 400 MM IC SHG, a single cavity Q-switched Nd:YAG laser, 532 $\mathrm{nm}$ wavelength, pulse duration $140 \mathrm{~ns}$, pulse energy $6.4 \mathrm{~mJ}$ ) at $10 \mathrm{kHz}$ and used a frequency divider to reduce the frequency by a factor of 3 , resulting in $3.3 \mathrm{kHz}$ pulses. The cameras and laser were triggered externally and set to synchronize on the rising edge of a periodic signal, created by a wave-form generator. The time series data of six seconds was stored in the camera's internal memory and saved after the recording finished. 


\section{PIV Aerosol Generation and Injection}

I chose to use smoke as the seeding material since these particles are relatively simple to fluidize, and provide the smallest possible sizes ( $<1$ micrometer) and densities (about $1 \mathrm{~g}$

$\mathrm{cm}^{-3}$ ), which is needed to approximate passive tracers in the dilute carrier phase. I opted to generate the smoke by burning material, rather than using an oil-based fog generator because I preferred that the seeding material would not condense on surfaces inside the apparatus and the tubing leading to it. I introduced the smoke through the perforated ring shown in figure 3.5. The sealed buffer vessel (flask) used to contain the smoke is shown in figure 3.11. I used two different ways of generating the smoke. In one image, I prefilled the flask with smoke by siphoning water out of it, which sucks in the smoke and allows it to be stored for several minutes and later connected to vacuum tubing leading to the apparatus. In the other image, I avoided using water and let the vacuum created by the apparatus itself suck the smoke into the flask and through the vacuum tubing. The flask was plugged, except for a small glass funnel leading out of it, where I inserted an herbal (patchouli) cigarette. Opening the valve to the apparatus created a strong pressure gradient which drew in smoke from the cigarette within a few $\times 10$ seconds. I set the valve to create a 10 mbar value of SS2 (see figure 3.6). Both methods create a resevoir of smoke, but I used the latter approach because it is less time consuming than the siphoning proceedure. 


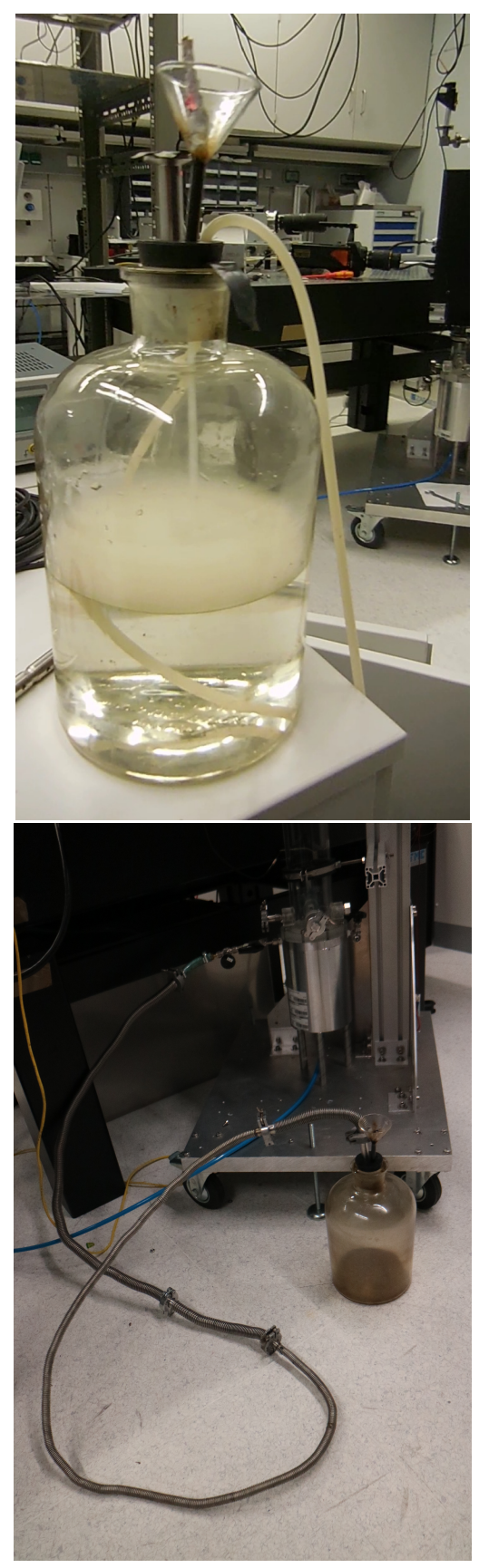

Figure 3.11.: Altenative methods to generate smoke. Top: by siphoning water out of the sealed buffer vessel. Bottom: by using the vacuum generated by the apparatus. The long vacuum tubing allows the buffer vessel to be hidden behind a protective laser curtain, for safety reasons. 
Note that while the flask I used is small, it is sufficient because the mass flow rate into the system is necessarily low in order to maintain steady 10 mbar pressure. For the same reason, it was not possible to conduct PIV measurements at even further reduced pressure, since it would have meant reducing the flow rate even further and in so doing, decreasing the particle seeding density to levels unsuitable for PIV measurements. One could devise alternative injection methods, but it would be impractical, for example, to generate particles inside the vessel itself. An alternative might be to accept a low particle seeding density and to do $2 \mathrm{D}$ particle tracking instead. However, this approach might require a lot of data to reach statistical convergence in calculating the mean flow profile.

Figure 3.12 shows a raw image, where the $\mathrm{x}$ coordinate corresponds to the horizontal direction and y to the vertical. The black and white image has been inverted so the particles can be discerned. The length of the interrogation window is shown on the scale bar, corresponding to $2.4 \mathrm{~mm}$ in physical units. The vertical patterns are internal reflections of the laser sheet in the cylindrical tube. Those areas are masked in the processing, shown in figure 3.13. PIV processing was performed with DaViS 7.3 software. Image preprocessing consisted of a mean background image subtraction, followed by a local non-linear subtractsilding-minimum filter over a scale of 64 pixels, linear 3x3 pixel Gaussian smoothing filter and intensity renormalisation filter. Preprocessed images were interrogated with a 3-pass PIV scheme, using 128x128 px windows at 50\% overlap for the first pass and 64x64 px windows at 50\% overlap for the subsequent passes. The maximum particle displacement was around 18 pixels. The average seeding concentration was 0.0024 particles per pixel. 


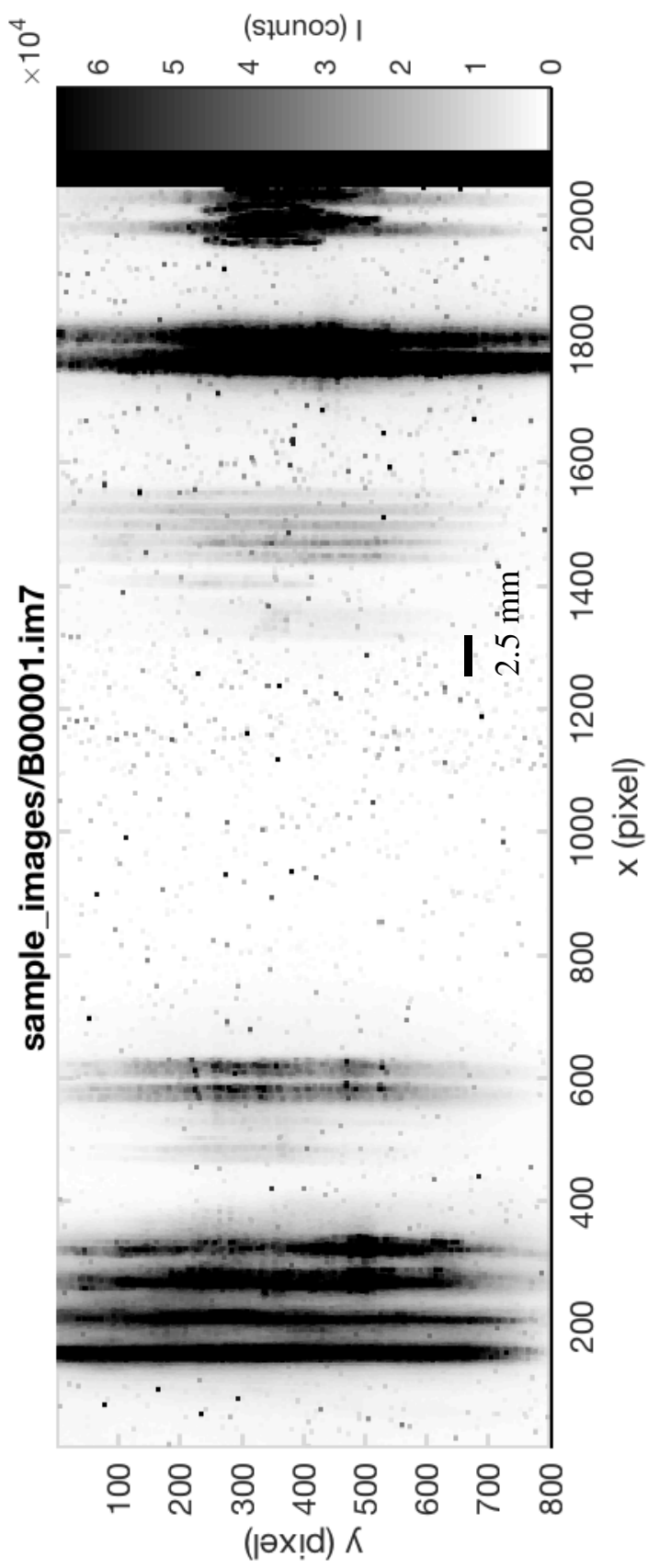

Figure 3.12.: Raw image. $\mathrm{Y}$ is vertical and $\mathrm{X}$ is horizontal. Fringes are internal reflections of the laser sheet. 


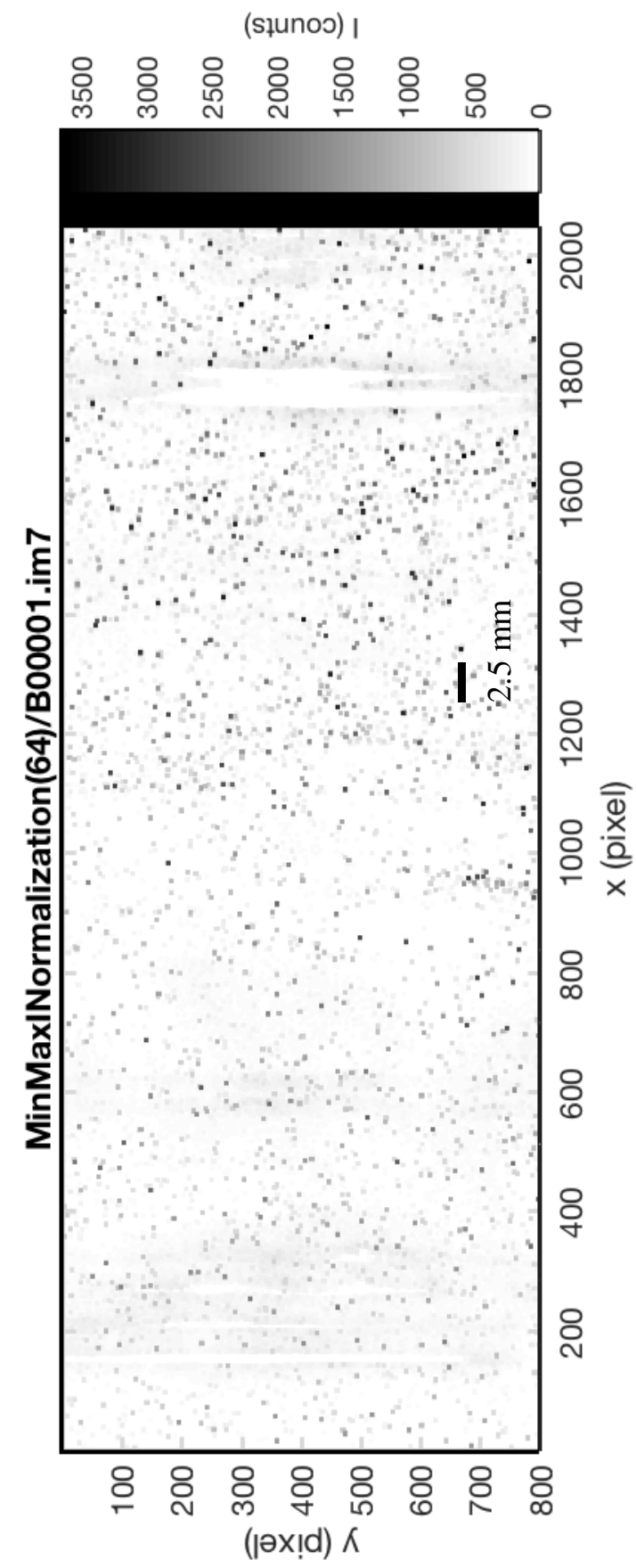

Figure 3.13.: Image after processing. $\mathrm{Y}$ is vertical and $\mathrm{X}$ is horizontal. The fringes from the pre-processed data (see figure 3.12) have been masked and the veocity field is calculated by interpolation over this region. 


\section{Two-Dimensional Flow Field and Mean Profile}

I generated vector fields from subsequent image pairs in the six-second long time-series PIV recording. The mean vector field is shown in figure 3.14. The dominant component is, unsurprisingly, the vertical one and its magnitude is around $1.4 \mathrm{~m} / \mathrm{s}$ in the center and decreases to about $0.8 \mathrm{~m} / \mathrm{s}$ towards the edge of the measurement volume, which is roughly $60 \mathrm{~mm}$ wide (the measurement volume was cut to fit the size of the calibration mask; the thickness of the laser sheet was less than a millimeter.).

I derived the mean flow profile by taking a line profile of all vector fields used to produce the mean vector field shown in figure 3.14. Note that for this and other figures of the mean flow profile, I am reverting back to 3D coordinates, where $z$ is vertical, and $x$ and $y$ are horizontal. The blue dots are the measured values and the purple line is a parabolic fit to the mean values of all the data, extrapolated to a radius corresponding to the container walls. The discrete sampling in the radial direction reflects the resolution of the vector field. The maximum of the parabola is approximately $6 \mathrm{~mm}$ off-center in field of view and I applied a shift by this amount to center the curve in the figure. The fact that the flow approaches zero at the walls is expected for a Poiseuille profile. 


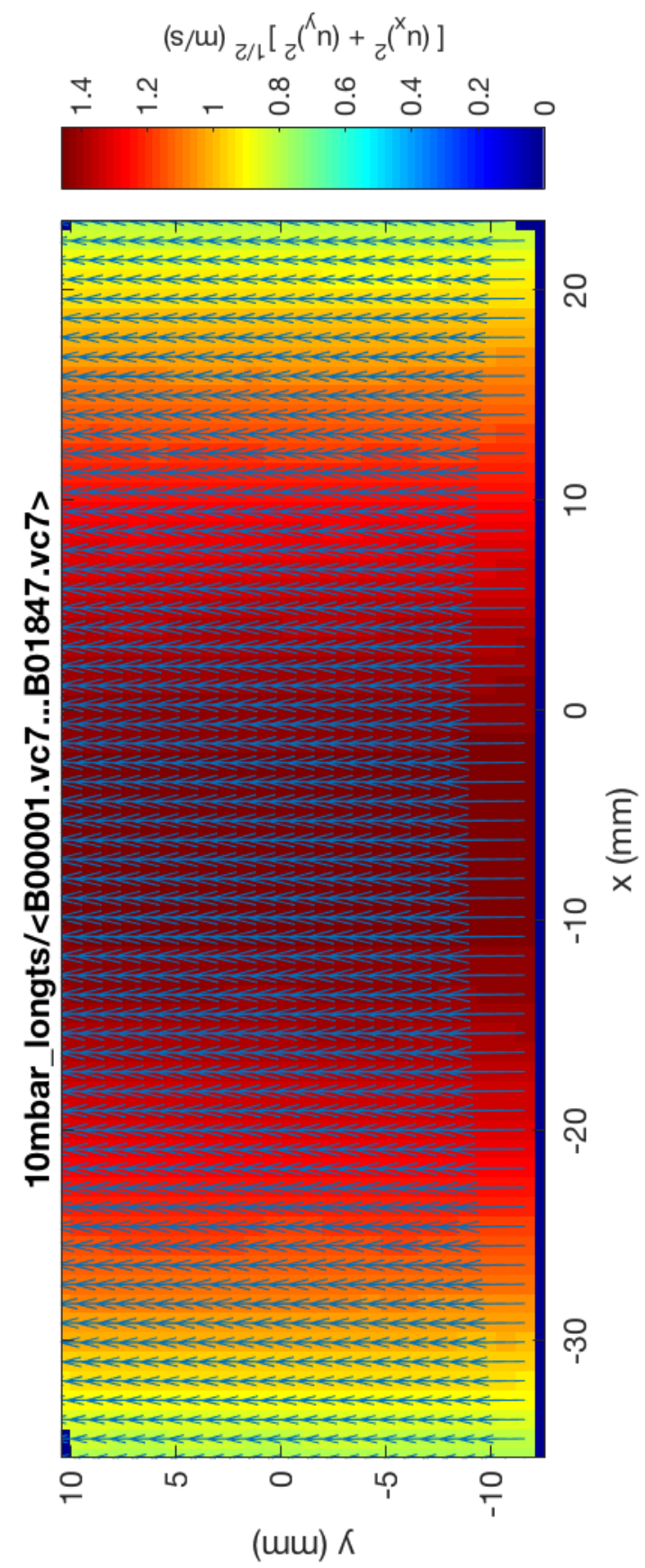

Figure 3.14.: Mean vector field from 6-second time series PIV data. 
The region in which the inertial particle measurements were made is smaller, because the cameras should run at high speed and so the resolution must be reduced. Furthermore, in a stereoscopic camera arrangement, the measurement volume is determined by the region in which the depth of field from all three cameras overlap, further trimming the field of view of the individual cameras. In the measurements described in the next section, the observation volume is approximately $1.3 \mathrm{~cm}^{3}$, located close to the center of the tube. 


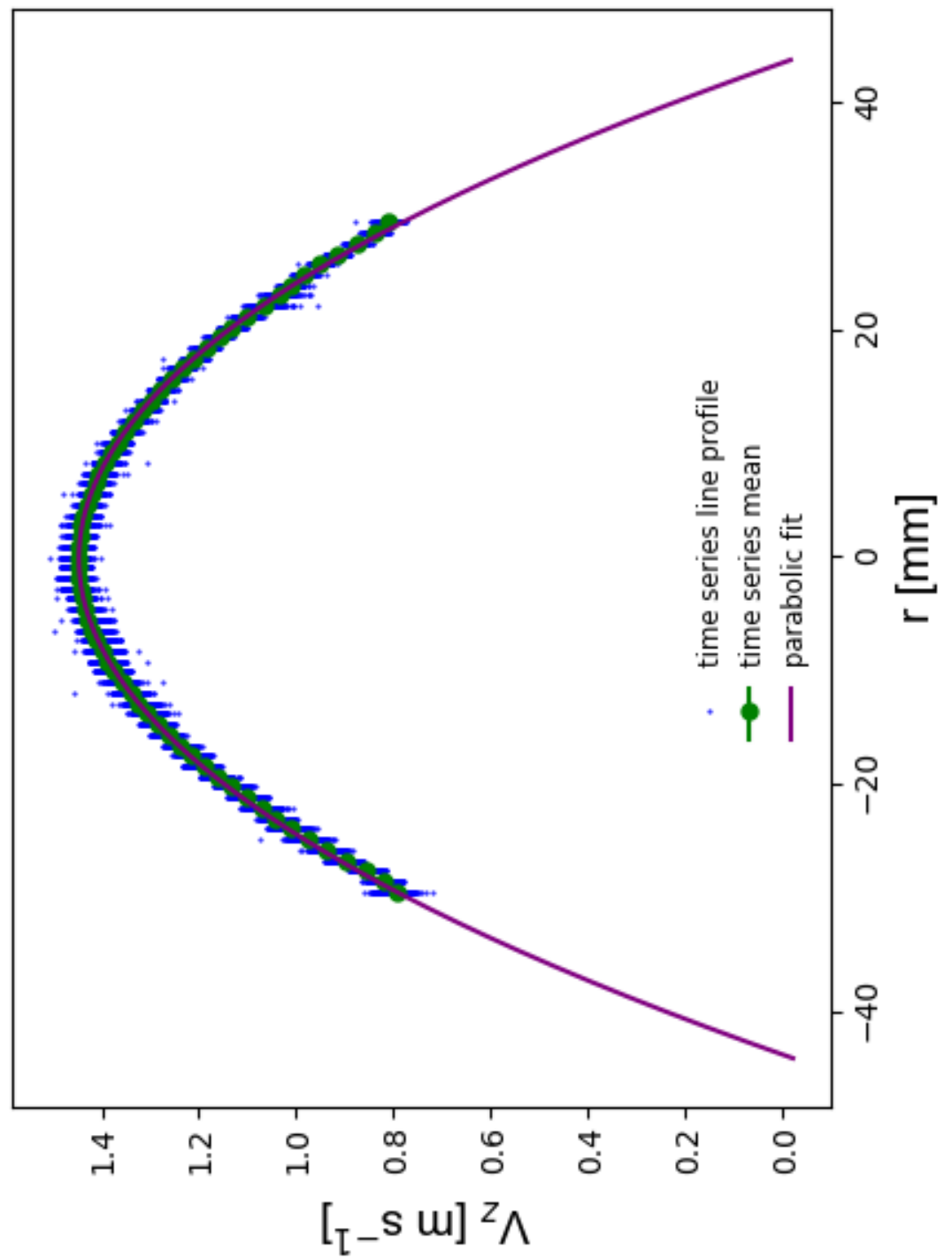

Figure 3.15.: Blue dots represent the line profiles of 6-seconds of time series PIV vector fields. The mean of the time series data is overplotted in green and the fit to the mean profile is shown in purple. The parabolic fit is extrapolated to match the $90 \mathrm{~mm}$ tube diameter. 


\subsection{Disperse Phase}

\subsubsection{Inertial-particle Staging}

Particles are preloaded into the chamber and become fluidised, forming a continuous vertical particle stream. The particle stream always has a net upward direction in the certerline, where the flow is fastest, but can fall downward where the flow is slower, closer to the walls. Once the particles reach the bottom, they are collected by the funnel insert and they float up again. Figure 3.4 shows a cut away of the apparatus base, containing a particle staging platform. It is composed of fine wire mesh and is secured at the bottom of a funnel-like insert. Air passing from the underside of the insert entrains the particles in the flow, provided that the gas velocity is faster than $u_{t}$ of the particles. The apparatus opperates at pressures at which the terminal velocity may depend upon the pressure. In figure $3.16, u_{t}\left(d_{p}, \rho_{g a s}\right)$ is plotted for three example pressures: 0.5,1.0 and 5.0 mbar. The dashed line is calculated using the Stokes drag law and the solid lines represent the Epstein drag (recall that Stokes drag is proportion to $d_{p}^{2}$ and Epstein drag directly to $d_{p}$ ). The point at which the three pressure-dependent lines intersect the dashed curve, corresponding to $9 / 2 \lambda$, happens for increasingly large particle diameter at higher pressures (not shown for 0.5 or $1.0 \mathrm{mbar}$ ). At 5 mbar, all particles $80 \mu \mathrm{m}$ and smaller are below the Stokes-Epstein division expressed by equation 1.9. Comparing the center-line velocity of the gas of $1.4 \mathrm{~ms}^{-1}$, taken from the calculation shown in figure 4.2 , to $u_{t}\left(d_{p}, \rho_{g a s}\right)$ delineates the rough pressure region at which the gas velocity will be sufficient to suspend the particles; $u_{\text {gas }} \geq u_{t}\left(d_{p}, \rho_{\text {gas }}\right)$ is between 1 and 5 mbar for steel particles of $d_{p}=15-65 \mu \mathrm{m}$. 


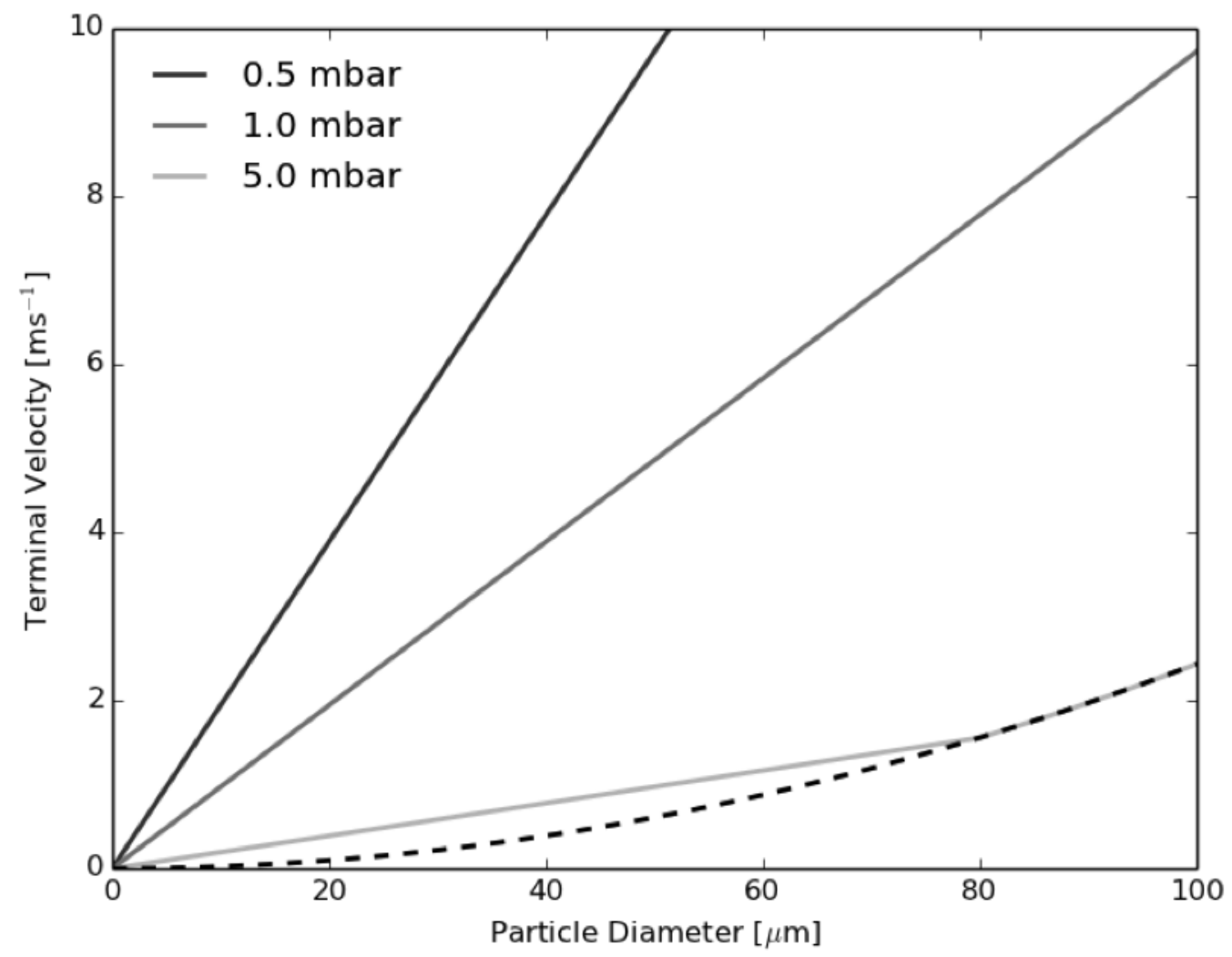

Figure 3.16.: Terminal velocity of steel particles, $\rho_{p}=8050 \mathrm{~kg} \mathrm{~m}^{-3}$, vs. particle size for three different operational pressures. Dashed lines represent the stokes drag law and solid lines the Epstein drag law. 
The pressure at which particles will float and that at which they cannot is bridged by the transition from SS1 to SS2 (see figure 3.6). During SS1, the particles sit on the staging platform $^{8}$. That is, the pressure of SS1 corresponds to too high of a terminal velocity for the particles to float. The point labeled 'equilibration' in figure 3.6 is when the particles first couple to the flow. The particle stream circulates for an indefinite amount of time during SS2. The particles are able to circulate because the gas velocity decreases closer to the walls, as shown in figure 3.15, and so particles that migrate out of the centerline of the flow fall down to the bottom of the container. There, they are collected by the tapered tray and land on the mesh staging platform, where they can be entrained in the flow again.

In practice, I find that the lowest pressure at which I can suspend solid particles is for a SS2 between 2.5 and 3 mbar, consistent with the above considerations.

\footnotetext{
${ }^{8}$ They are perturbed and float during the initial evacuation, but they settle back down even before the pressure reaches SS1.
} 


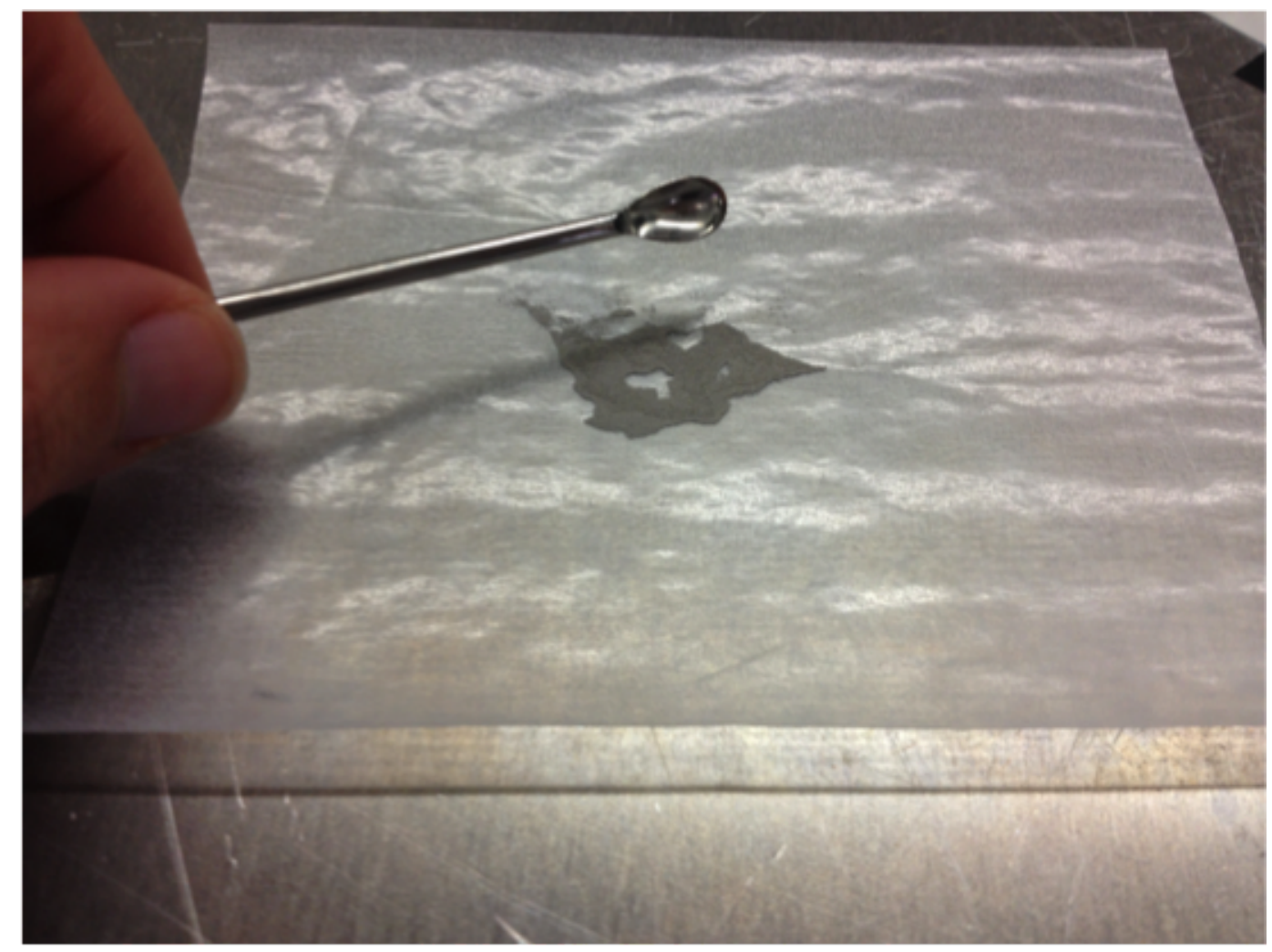

Figure 3.17.: Preparation of a sample of steel particles $15-65 \mu \mathrm{m}$, weighing a total of 120 $\mathrm{mg}$. 
The mass of the gas in the apparatus when the pressure is 1 mbar is 12 milligrams. To target a value of $\varepsilon$ around 1, I weighed the particles before putting them onto the staging platform. They are injected while the system is at ambient conditions by putting them into a pipette whose tip is inserted at $\mathrm{H} 1$. Blowing lightly compressed air through the pipette blows the particles into the chamber. Because I found that the flow apparently selects a subsection of the particle size distribution (demonstrated in the next chapter), I introduced an amount closer to 120 milligrams. Figure 3.17 shows $120 \mathrm{mg}$ of steel particles. Although the system is mainly closed, I found that the particle count could diminish ${ }^{9}$, requiring me to add more particles. For all of these reasons, the bacground value of $\varepsilon$ can only be moderately controlled. However, it can be measured easily a posteriori from the particle-tracking data. In the next chapter I report on the value of $\varepsilon$ achived in the experiments.

\subsubsection{Particle Containment}

Particles that reach the top of the sedimentation vessel, rather than sedimenting along the sides of the walls, enter an expansion unit at the top of the tube (indicated in figure 3.1 and 3.2). In the expansion, the tube cross section increases and therefore the gas velocity decreases. There is also a fine metallic mesh screen at the top of the expansion, to prevent any stray particles from continuing upstream to the vacuum pump.

To decide upon the dimensions of the expansion, I updated the velocity calculation of a single particle of typical size and density in our experiments as it passed through the hollow cone, of height $l$ and opening angle $\gamma$. The momentum equation for a single sphere with a single velocity component $u_{\mathrm{pz}}$, given by equation 1.16 , can be solved analytically, if the gas velocity is held constant. Therefore, I first considered a simple force balance between gravity

\footnotetext{
${ }^{9}$ Most likely because they lodge in the corners of the apparatus' joints.
} 
and Stokes drag, with $\mathrm{g}=-9.8 \mathrm{~m} / \mathrm{s}$,

$$
\begin{gathered}
m_{\mathrm{p}} \frac{d u_{\mathrm{pz}}}{d t}=m_{\mathrm{p}} g+F_{\mathrm{d}}, \\
\frac{d u_{\mathrm{pz}}}{d t}=\frac{u_{\mathrm{g}}}{T_{f}}-\frac{u_{\mathrm{pz}}}{T_{f}}+g,
\end{gathered}
$$

which has the solution:

$$
\begin{array}{r}
u_{\mathrm{pz}}=e^{\frac{t}{T_{f}}}\left[\int e^{\frac{-t}{T_{f}}}\left(\frac{u_{\mathrm{g}}}{T_{f}}+g\right) d t+c\right] \\
=e^{\frac{t}{T_{f}}}\left[-T_{f} e^{\frac{-t}{T_{f}}}\left(\frac{u_{\mathrm{g}}}{T_{f}}+g\right)\right]+c e^{\frac{t}{T_{f}}} \\
=-\left(T_{f} g+u_{\mathrm{g}}\right)+c e^{\frac{t}{T_{f}}} .
\end{array}
$$

The integrating constant $c=T_{f} g+u_{\mathrm{g}}$ when $v_{\mathrm{p}}=0$ at $t=0$ and therefore:

$$
u_{\mathrm{p}}=\left(T_{f} g+u_{\mathrm{g}}\right)\left(e^{\frac{-t}{T_{f}}}-1\right)
$$

which reduces to equation 1.11 as $t$ becomes large in a static fluid. As the particle travels upwards in the expansion chamber, however, it finds itself at a new value of $u_{\text {gas }}$, given by equation 3.2, replacing $h$ with $l$ and with $a(l)$ depending upon $\gamma$. In order to use the simple analytic expression of 3.6 , I conceptually subdivided the cone into horizontal slices, $\delta l$, corresponding to discrete time steps $\delta t$. I made the assumption that the gas velocity within a given slice would be constant and therefore the initial conditions of the particle and gas velocities at each time could be given by their values at the previous time step, provided that $\delta t>T_{f}$. This way, I updated $c$ at every time step and stopped the calculation when $u_{\mathrm{pz}}$ reached zero, returning the value of $l$ for which this occurred. I varied $\gamma$ and limited $l$ based upon practical constraints, i.e. it would not be possible to weld the cone onto its supportive 
base if the angle were greater than $\sim 30^{\circ}$ and the installation would be feasible for $l$ equal to a few $\times 10 \mathrm{~cm}$. Note that the derivation above holds for particles in the Epstein drag regime as well, however a much faster gas flow is required for the same size particles in the Stokes regime.

\subsubsection{Particle Tracking}

To study the particles' dynamics, I track their positions in time using the stereoscopic camera arrangement shown in figure 3.1 and from the positions derive the velocities and accelerations of the particles at all times. The first and second derivatives of the position are calculated using a finite difference scheme, convolved with a gaussian filter. While particle tracking done in water with polystyrene particles, or performed on water droplets might use backscattered light as the illumination source, I use backlighting to image my solid steel particles in shadow, using three 10-Watt light emitting diode (LED) spotlights. The LED beams are expanded using lenses of diameter and focal length of $75 \mathrm{~mm}$ and $85 \mathrm{~mm}$, respectively. A closeup of the spotlight and lens, mounted on optical posts, is shown in figure 3.18.

The centerline gas flow speed of $1.4 \mathrm{~m} / \mathrm{s}$ measured by the PIV experiments is fast; particles coupled to the flow are expected to travel a significant fraction of this velocity - at least 20-50 $\mathrm{cm} / \mathrm{s}$. Therefore I used high-speed phantom v10 cameras at a framerate of $2000 \mathrm{~s}^{-1}$. The cameras were set upon a leveled optical table and I designed custom mounts for them, shown in figure 3.19. The camera mounts have translational freedom on three axis and rotational freedom in one plane. Adjustments to the heightcan be made by loosening the optical posts upon which they sit and turning the threaded bolster on the underside of the mounting plate. The camera appertures are supported from below by a piece of aluminium that extends from the mounting plate. An optional feature is to use brackets to mount the camera sideways, in 
case one wants to view the particle stream in landscape ${ }^{10}$.

\footnotetext{
${ }^{10}$ This configuration was used for initial tests using the Phantom 65 camera, in order to exploit the camera's large chip in viewing the centerline of the flow. The Phantom 65 cameras have slower recording speeds than the Phantom 10, however, so they were not used for the experiments.
} 


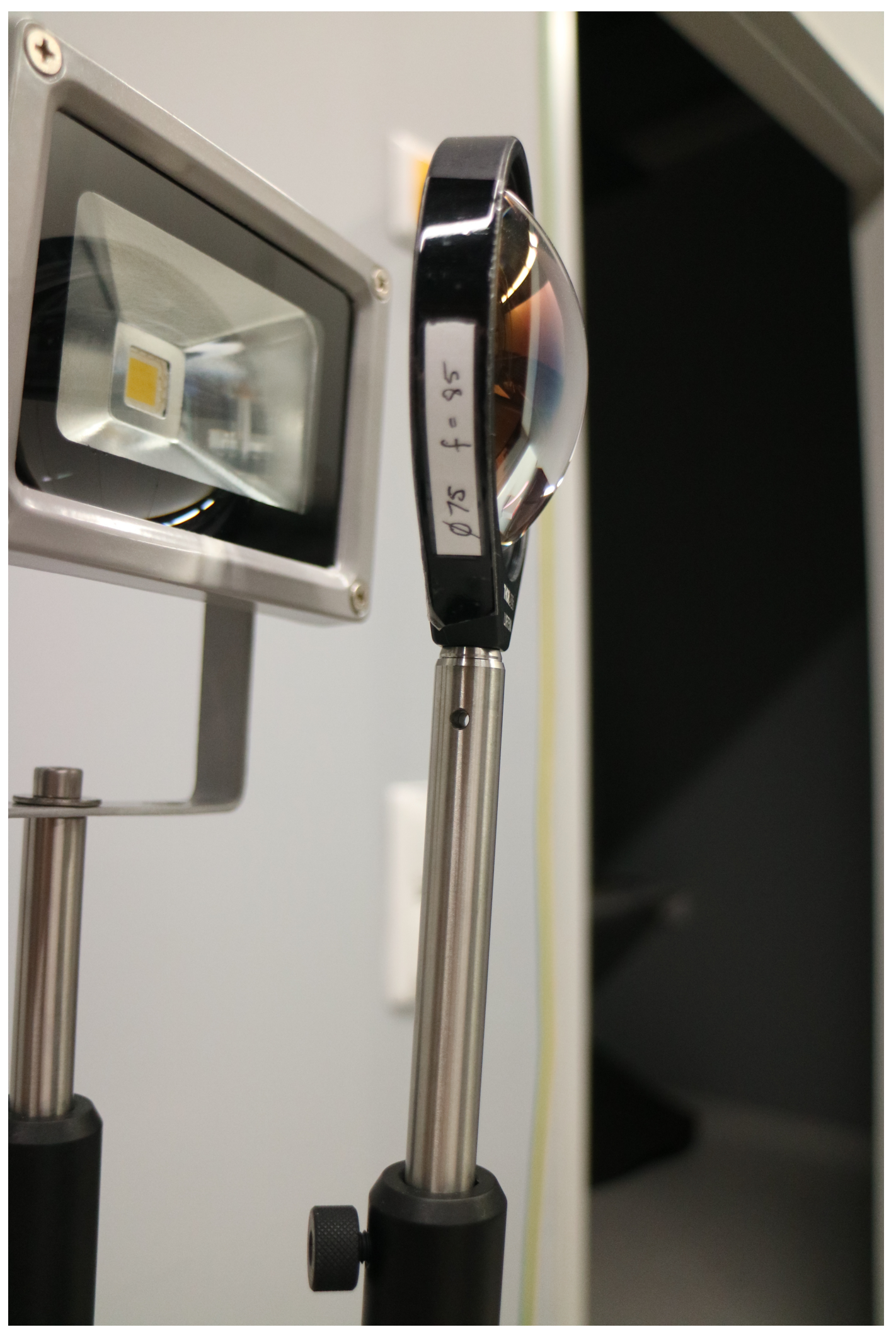

Figure 3.18.: 10-Watt LED lamp with beam-expanding lens. Used as backlighting for Lagrangian Particle Tracking. 


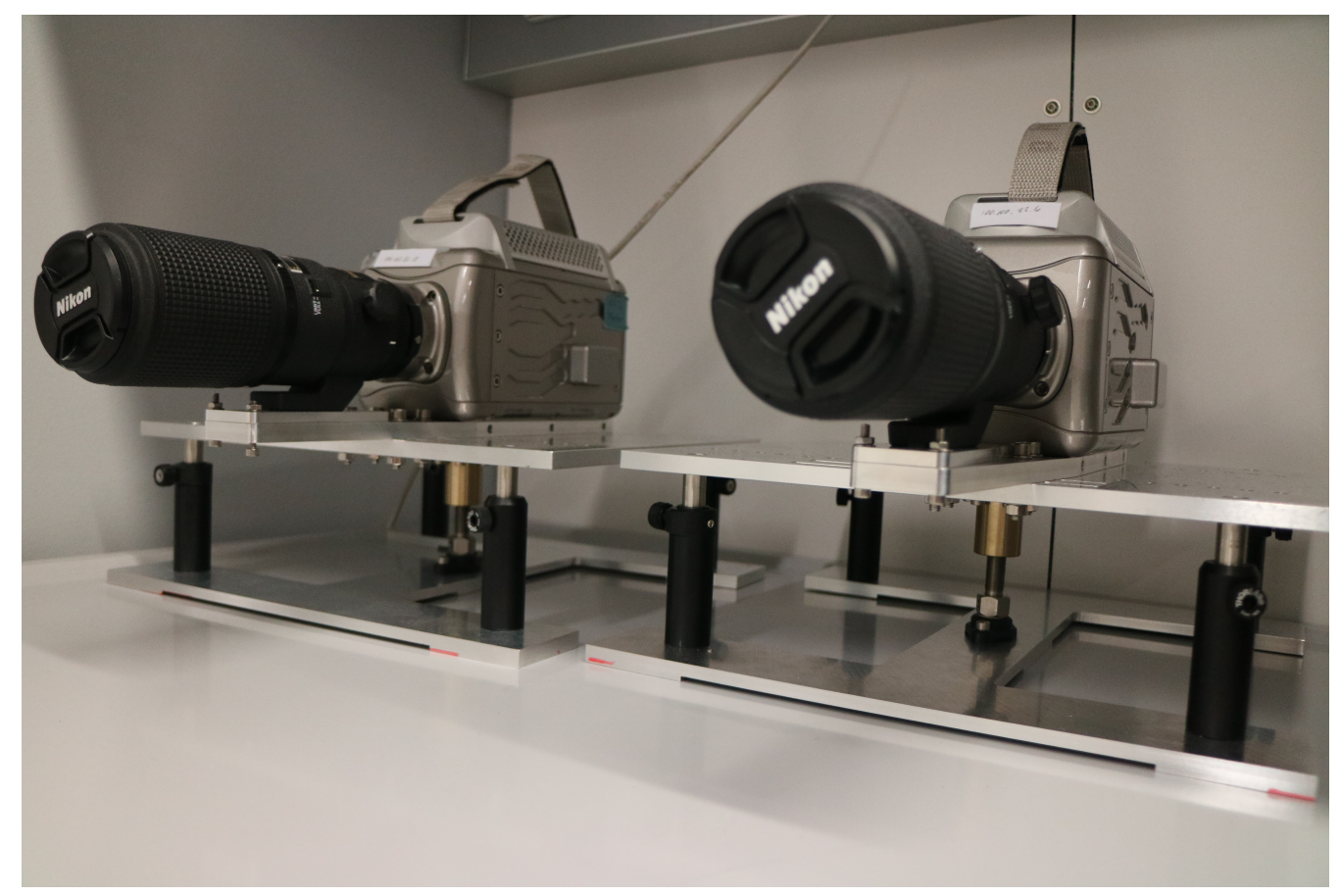

Figure 3.19.: Camera mounts with translational and rotation freedom. Holding Phantom 10 cameras that are fitted with 200mm Macro Nikon apertures.

The particle tracking algorithm that produced the particle trajectories analyzed in the Experimental Results chapter is a variation on the standard particle tracking procedure [Ouellette 2006 ] consisting of the following steps in the stated order: particle image finding, stereoscopic reconstruction, tracking in time. However, each of these steps has been changed from the originally stated version to fit the specific needs and limitations of the current experimental setup. Below I describe each step in more detail ${ }^{11}$.

\subsubsection{Particle Image Finding}

The first step of the tracking algorithm is a precise localization of the particle images on each camera sensor. For well-lit and well-focused particle images produced for example by laser

\footnotetext{
${ }^{11}$ The remainder of this section closely follows notes written by Jan Molacek describing the customized camera model that he implemented for this project.
} 
illumination of tracer particles in water, one can choose from a number of methods such as center-of-mass, 1-D gaussian fit, 2-D gaussian fit, or logarithmic fit. The particular choice of method is determined by the relative value placed on speed as opposed to accuracy. In the present setup, due to the comparably weak illumination producing a low signal to noise ratio, and the broad form of the point spread function, only the 2-D gaussian fit method is capable of reliably extracting the particle image positions. Figure 3.20 shows a single particle in shadow, its intensity map, and slices in the intensity. 




Figure 3.20.: Top: shadowgraph of a steel particle. Center: Intensity map of the particle. Bottom, slices in intensity, the legend shows the y-position of the slice. The spatial resolution is $\sim 12 \mu \mathrm{m} /$ pixel. 
First, the raw video is read once in full to determine the median intensity of each pixel, which is considered to be a sufficiently good approximation to the background intensity due to the relatively low particle density used. Next, the video is read once more, but this time the pixel intensity of each frame is subtracted from the median pixel intensity to obtain the processed frame, which should only show the intensity variation due to particle presence, with particle locations corresponding to local intensity maxima. All local intensity maxima above a given threshold (in our setup the threshold value was chosen to be 10) are considered potential approximate locations of particle images. For each such local maximum, a square window of side length 13 pixels and centered on the local maximum is selected as the set of pixels on which the fitting (see below) will be performed. In case that local maximum is less than 7 pixels away from the frame boundary, the square window is appropriately cropped to fit inside the video frame.

I try to fit the pixel intensity profile with the following function of 6 parameters (in its most general form):

$$
f(x)=A \exp \left\{\kappa_{4}\left(x-x_{0}\right)^{2}+\kappa_{5}\left(x-x_{0}\right)\left(y-y_{0}\right)+\kappa_{6}\left(y-y_{0}\right)^{2}\right\} \quad,
$$

where $x=[x, y]$ denotes the pixel position on the camera sensor. I assume that $f(x) \rightarrow 0$ for $|x| \rightarrow \infty$, which implies $\kappa_{4}, \kappa_{6}<0$ and $\kappa_{4} \kappa_{6}-\kappa_{5}^{2} / 4>0$.

Let us denote the number of pixels within the fitting set described above as $N$, the location of $n$-th pixel from the fitting set as $\left[x_{n}, y_{n}\right]$, and its intensity $p_{n}$. To simplify the subsequent algebra, I also introduce for each pixel a vector $g_{n}=\left[1, \bar{x}_{n}, \bar{y}_{n}, \bar{x}_{n}^{2}, \bar{x}_{n} \bar{y}_{n}, \bar{y}_{n}^{2}\right]$ with $\bar{x}_{n}=x_{n}-x_{0}$, $\bar{y}_{n}=y_{n}-y_{0}$ and write $g_{n i}$ for the $i$-th component of $g_{n}$. Thus I can write the value of the fitting function at the location of $n$-th pixel as

$$
f_{n}=\exp \left(\sum_{i=1}^{6} \kappa_{i} g_{n i}\right)
$$


with $\kappa_{2}=\kappa_{3}=0$ (this constraint effectively defines $x_{0}$ and $y_{0}$ ).

The best fit is then defined as that combination of parameters $\kappa_{i}, x_{0}$, and $y_{0}$ which minimizes the sum

$$
\sum_{n=1}^{N}\left[\exp \left(\sum_{i=1}^{6} \kappa_{i} g_{n i}\right)-p_{n}\right]^{2} \equiv \sum_{n=1}^{N}\left[f_{n}-p_{n}\right]^{2}
$$

In order to obtain an initial estimate of the fitting parameters that is needed for the subsequent iterative refinement, I use the first two moments of pixel intensities over the fitting set. For an intensity profile given by (3.8), I have

$$
\begin{aligned}
S_{0} & \equiv \int f(x) \mathrm{d} S=\pi A /\left[\kappa_{4} \kappa_{6}-\kappa_{5}^{2} / 4\right]^{1 / 2}, \\
S_{i} & \equiv \int f(x) x_{i} \mathrm{~d} S=x_{i} S_{0}, \\
S_{i j} & \equiv \int f(x)\left(x-x_{0}\right)_{i}\left(x-x_{0}\right)_{j} \mathrm{~d} S=\frac{1}{4} \pi A \Lambda_{i j} /\left[\kappa_{4} \kappa_{6}-\kappa_{5}^{2} / 4\right]^{3 / 2},
\end{aligned}
$$

where $\left[\Lambda_{x x}, \Lambda_{x y}, \Lambda_{y y}\right]=\left[2 \kappa_{6},-\kappa_{5}, 2 \kappa_{4}\right]$. By defining $D \equiv \kappa_{4} \kappa_{6}-\kappa_{5}^{2} / 4=S_{0}^{2} / 4\left(S_{x x} S_{y y}-S_{x y}^{2}\right)$, I can thus obtain the fitting parameters in the following manner: $x_{i}=S_{i} / S_{0}, A=S_{0} D^{1 / 2} / \pi$, and $\Lambda_{i j}=4 S_{i j} D / S_{0}$, from which $\kappa_{i}$ trivially follow. Thus the initial guess of the fitting parameters is achieved by calculating the following integrals,

$$
\begin{aligned}
\bar{S}_{0} & \equiv \sum_{n=1}^{N} p_{n}, \\
\bar{S}_{i} & \equiv \sum_{n=1}^{N} p_{n}\left(x_{n}\right)_{i}, \\
\bar{S}_{i j} & \equiv \sum_{n=1}^{N} p_{n}\left(x_{n}-x_{0}\right)_{i}\left(x_{n}-x_{0}\right)_{j},
\end{aligned}
$$

and setting $\left(x_{0}\right)_{i}=\bar{S}_{i} / \bar{S}_{0}, \bar{D}=\bar{S}_{0}^{2} / 4\left(\bar{S}_{x x} \bar{S}_{y y}-\bar{S}_{x y}^{2}\right), A=\bar{S}_{0} \bar{D}^{1 / 2} / \pi, \kappa_{4}=2 \bar{S}_{y y} \bar{D} / \bar{S}_{0}, \kappa_{5}=$ $-4 \bar{S}_{x y} \bar{D} / \bar{S}_{0}$, and $\kappa_{6}=2 \bar{S}_{x x} \bar{D} / \bar{S}_{0}$.

Given a starting guess for the fitting parameters $\kappa=\kappa^{m}$ (with the initial guess $\kappa^{0}$ obtained 
via the procedure described above), I need a way to refine the guess to further reduce the sum (3.10). Consider introducing a prefactor in (3.10):

$$
\sum_{n=1}^{N}\left[\left(\sum_{i=1}^{6} c_{i} g_{n i}\right) \exp \left\{\sum_{i=1}^{6} \kappa_{i}^{m} g_{n i}\right\}-p_{n}\right]^{2} \equiv \sum_{n=1}^{N}\left[\left(\sum_{i=1}^{6} c_{i} g_{n i}\right) f_{n}-p_{n}\right]^{2}
$$

I want to find those $c_{i}$ which minimise the sum in (3.13). This is achieved by differentiating (3.13) with respect to each $c_{q}$, giving

$$
\sum_{n=1}^{N} \sum_{i=1}^{6} c_{i} g_{n i} g_{n q} f_{n}^{2}=\sum_{n=1}^{N} g_{n q} f_{n} p_{n}
$$

which means that $c$ solves the system

$$
\tilde{M} c=\tilde{R} \quad \text { with } \quad \tilde{M}_{i j}=\sum_{n=1}^{N} f_{n}^{2} g_{n i} g_{n j} \text { and } \tilde{R}_{i}=\sum_{n=1}^{N} f_{n} p_{n} g_{n i} .
$$

Once $c$ is obtained, an improved guess for $\kappa$ given starting guess $\kappa^{m}$ is obtained by $\kappa_{i}^{m+1}=$ $\kappa_{i}^{m}+c_{i} / c_{1}$ for $i \neq 1$ and $\kappa_{1}^{m+1}=\kappa_{1}^{m}+\ln c_{1}$, since

$$
\left(\sum c_{i} g_{n i}\right) \exp \left\{\sum \kappa_{i}^{m} g_{n i}\right\} \approx c_{1} \exp \left\{\sum_{i=2} \frac{c_{i}}{c_{1}} g_{n i}+\sum \kappa_{i}^{m} g_{n i}\right\}
$$

In the above I have assumed that the correction $c$ is small, so that $\left|c_{i} / c_{1}\right| \ll 1$ for $i>1$. The improved estimate of $x_{0}$ is then obtained by again enforcing $\kappa_{2}^{m+1}=\kappa_{3}^{m+1}=0$.

The iterative procedure is run repeatedly until either the fitting parameters converge to within the desired precision, the fitting parameters diverge, or the number of iterative steps reaches a set limit. In the last two cases, I discard the results and do not consider the given local intensity maximum as a particle image location. 


\subsection{Stereoscopic Reconstruction}

In this step, I have obtained the particle image locations in each camera sensor and want to deduce the particle locations in three-dimensional space. Let us denote the particle image locations on the $c$-th camera sensor as $x_{n}^{c}$ with $1 \leq n \leq N^{c}$, where $N^{c}$ is the number of particle images obtained for the $c$-th camera. Given a map $\Pi^{c}$ from the three-dimensional world coordinate system onto the $c$-th camera sensor, for each combination of particle images $\left\{x_{i_{c}}^{c}\right\}$ I define the best stereoscopic fit $X_{\mathrm{BSF}}$ as the point in world coordinates that minimises the difference between its projections onto each camera sensor and the corresponding particle image in the least-square sense:

$$
E_{\Delta} \equiv \min _{X \in \mathbb{R}^{3}} \sum_{c=1}^{3}\left\|\Pi^{c}(X)-x_{i_{c}}^{c}\right\|^{2}=\sum_{c=1}^{3}\left\|\Pi^{c}\left(X_{\mathrm{BSF}}\right)-x_{i_{c}}^{c}\right\|^{2}
$$

The quantity $E_{\Delta}$ shall be called the triangulation error and has dimensions of $(\text { pixel })^{2}$. In the above equation I have assumed, as I will for the rest of the section, that the total number of cameras used is 3 , although the equations can be straightforwardly generalised to an arbitrary camera number.

Utilising some geometric properties of the mappings $\Pi^{c}$, one can efficiently search through all combinations of particle images $\left\{x_{i_{c}}^{c}\right\}$ that yield small triangulation errors. All combinations giving triangulation error below a given threshold (typically on the order of a few $(\text { pixel })^{2}$ ) are considered as images of a single particle, and the corresponding $X_{\mathrm{BSF}}$ are added to the set of triangulated points.

The main difficulty of the stereoscopic reconstruction lies in establishing a simple yet accurate mapping $\Pi^{c}$. A necessary first step towards that goal lies in a physical calibration procedure, during which a set of calibration points of precisely known position in the world coordinates are imaged in the same manner as the particles during experimental runs. Thus the mapping $\Pi^{c}$ is determined at least for a discrete set of points ideally spanning in a 
more or less uniform manner the whole measurement volume. In our case the calibration procedure consisted of imaging a high-precision calibration slide containing a rectangular grid of hollow square markers, shown in Fig. 3.21, whose centers were used as the calibration points. The grid period in both directions was $0.5 \mathrm{~mm}$ and the square markers had a side length of $0.375 \mathrm{~mm}$. Three of the square markers in the middle of the mask were filled instead of hollow, so that the precise location and orientation of the calibration slide could be established. The slide was clamped between two glass plates to ensure its flatness, and suspended into the glass cylinder by means of a long rod attached to a translation stage, which was used to move the calibration slide in increments of $2 \mathrm{~mm}$ normal to its plane to sample the whole measurement volume.

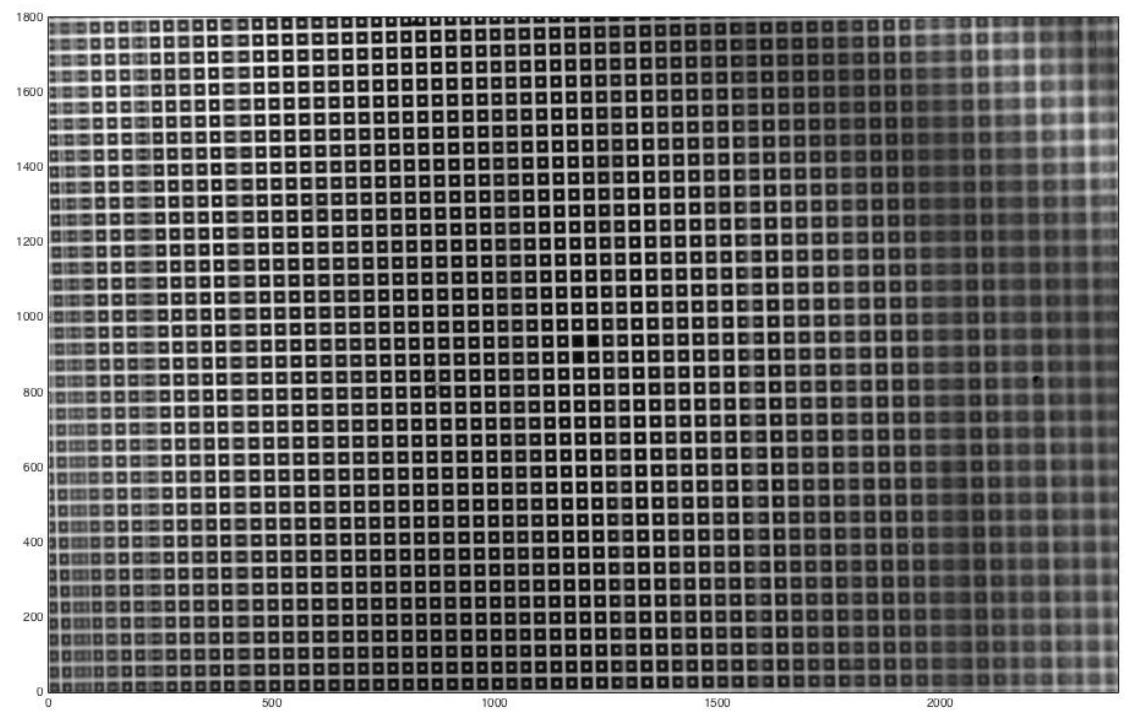

Figure 3.21.: Image of the calibration slide captured during the calibration procedure. The three filled squares mark the position of $x=z=0$.

Below are the results of a calibration performed on our experimental setup. Fig. 3.22 shows the (a) top- and (b) side-view of the volumes visible to each camera. The lines are the lines of sight (a set of points imaged onto a single point on the sensor) corresponding to the corners 
and the center of each sensor. Fig. 3.23 shows a detailed view of the measurement volume, defined as the set of points visible by all three cameras. It has the shape of an irregular hexagonal prism with a volume, henceforth $v_{\text {meas }} \mathrm{xof} 1364 \mathrm{~mm}^{3}$ and maximum diameter of $[13.3,20.2,9.1] \mathrm{mm}$ in the $[x, y, z]$-directions. One pixel displacement on the camera sensor corresponds to a displacement of at least $11.5 \mu \mathrm{m}, 12.9 \mu \mathrm{m}$, and $13.0 \mu \mathrm{m}$ in real space for camera 0,1 , and 2 respectively.
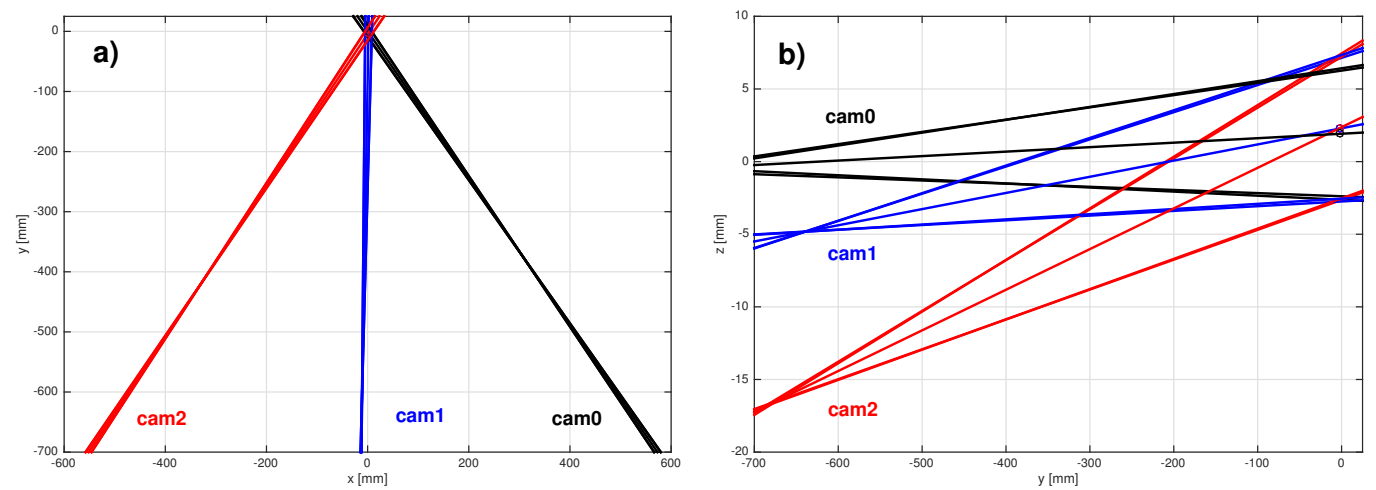

Figure 3.22.: (a) Top- and (b) side- view of the lines of sight corresponding to the corners and centerpoint of each camera sensor. The intersection of the lines of sight marks the location of the measurement volume.

Given the set of calibration points and their images, the next step is finding a smooth mapping with a reasonably simple functional form that closely fits to the mapping of the calibration points. The most commonly used mapping is the pinhole model where each point is projected onto the sensor along a straight line which goes through a special point (pinhole) that is common to all points. In a slightly generalised form, the pinhole model can be written as

$$
\Pi(X)=A+\frac{B X}{1-\mu n \cdot X} \quad \text { with } \quad B n=0 \quad \text { and } \quad|n|=1
$$

Here $A \in \mathbb{R}^{2 \times 1}, B \in \mathbb{R}^{2 \times 3}, n \in \mathbb{R}^{3 \times 1}$ and $\mu$ is a scalar. Often the pinhole model is supplemented by a corrective function that accounts for the spherical aberration of the lens or other lens distortion effects. A common functional form used is the radially symmetric 


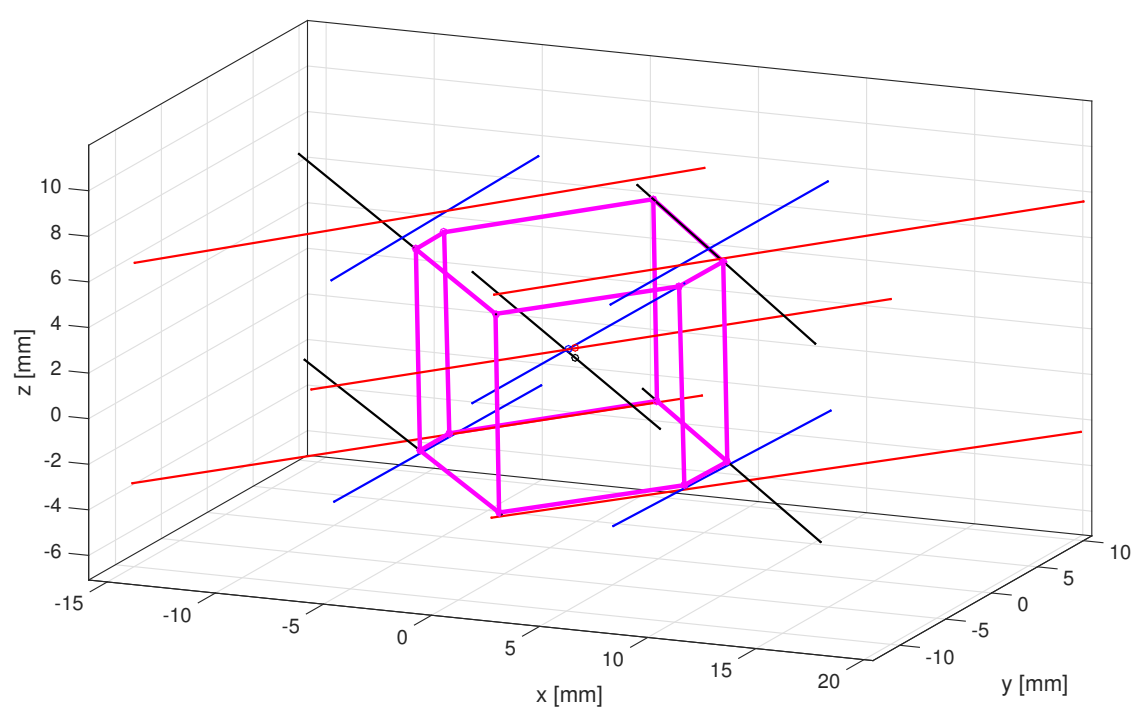

Figure 3.23.: A close-up view of the neighbourhood of the origin, with the lines of sight corresponding to the corners and centerpoint of each camera sensor, as in Fig. 3.22. The measurement volume, given by the intersection of lines of sight from all three sensors, is shown in thick purple lines.

two-parameter correction

$$
x_{\text {corr }}=x_{0}+\left(x-x_{0}\right)\left(1+k_{1} r^{2}+k_{2} r^{4}\right) \quad \text { where } \quad r=\left|x-x_{0}\right|
$$

where $x_{0}$ is the distortion center and $k_{1}, k_{2}$ the distortion parameters.

In our experimental setup, the presence of the cylindrical glass shell between the measurement volume and the cameras causes refraction of the incoming light in such a manner that renders the principal assumption of the pinhole model (common intersection point of all lines of sight) invalid. This is clearly visible in Fig. 3.24, where I show the intersection of lines of sight for one of the cameras used, with four different $x z$-planes. Intersections of 1600 lines of sight are shown, corresponding to a uniform rectangular grid of $40 \times 40$ sensor locations. In Fig. 3.24a, the intersections with a plane $y=4 \mathrm{~mm}$, close to the focal plane, are shown to be regularly spaced. In Fig. 3.24b and Fig. 3.24c, I show the planes for which the 
spread of the intersections is minimised in the $x$ - and $z$ - directions respectively, and finally, in Fig. 3.24d, I show the intersections with a general $x z$-plane $(y=-1000 \mathrm{~mm})$.
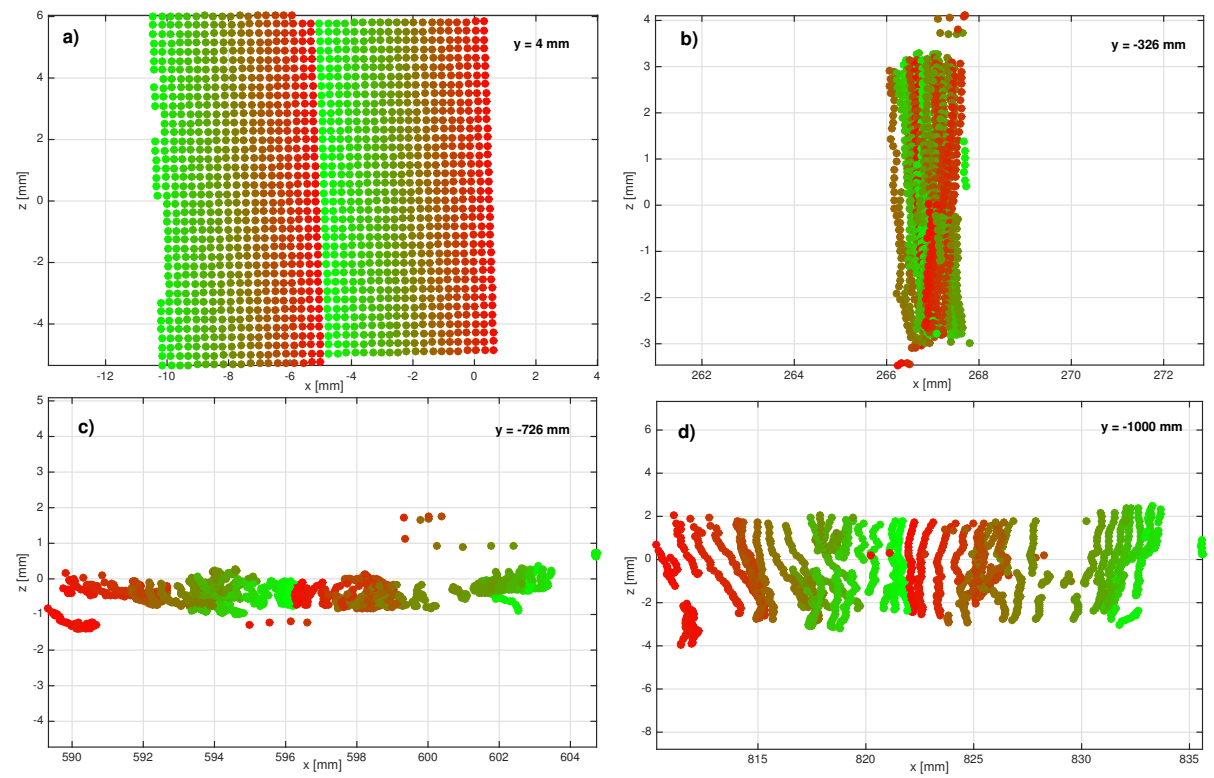

Figure 3.24.: Intersections of lines of sight, corresponding to a uniform rectangular grid of $40 \times 40$ sensor locations covering the entire sensor, with $x z$-planes at four different locations: Near the focal plane at $y=4 \mathrm{~mm}$ (a), the intersections are regularly spaced. At $y=-326 \mathrm{~mm}$ (b), they collapse to a thin vertical strip, whereas at $y=-726 \mathrm{~mm}$ (c) they collapse to a thin horizontal strip. The intersection distribution at $y=-1000 \mathrm{~mm}$ (d) is shown to illustrate the general case.

If the mapping were to be well-approximated by a pinhole model, I would expect the two minimal-spread planes to be located at similar distances from the focal plane. However, in the present case the distances are quite different from each other: $326 \mathrm{~mm}$ vs. $726 \mathrm{~mm}$ for camera $0,533 \mathrm{~mm}$ vs. $652 \mathrm{~mm}$ for camera 1, and $412 \mathrm{~mm}$ vs. $646 \mathrm{~mm}$ for camera 2. This complication can be captured by a simple generalisation of the pinhole model, which I call a two-slit model, and which assumes that all the lines of sight pass through two mutually perpendicular slits. In the present setup, due to the alignment of the camera sensor orientations with the vertical axis of the glass cylinder, the slits are aligned with the principal 
sensor axes, which simplifies the functional form of the two-slit model to

$$
\Pi(X)_{i}=A_{i}+\frac{B_{i j} X_{j}}{1-\mu_{i} n_{j} X_{j}} \quad \text { with } \quad B_{i j} n_{j}=0 \quad \text { and } \quad|n|=1
$$

Note that setting $\mu_{1}=\mu_{2}=\mu$ reduces the two-slit model to the pinhole model, as it physically means setting the two slits at the same distance from the origin and therefore constrains the lines of sight to pass through the slit intersection. The model coefficients are obtained iteratively using the calibration points $X^{m}$ and their images $x^{m}$ in the following manner: I start with the initial guess for $\mu=\mu^{(0)}=0$, which means that $A$ and $B$ are the linear fit coefficients of the map from $\left\{X^{m}\right\}$ to $\left\{x^{m}\right\}$. When at the $i$-th iterative step $A^{(i)}$ and $B^{(i)}$ are known, $n^{(i)}$ is found from the conditions $B^{(i)} n^{(i)}=0$ and $\left|n^{(i)}\right|=1$ (the orientation of $n$ is not important, it should only be kept consistent across the iterative steps). Finally, keeping $A, B$, and $n$ constant, $\mu$ is found by minimising the difference between mapped and observed calibration point images in the least-square sense. In the following iterative step I replace each $X_{j}^{m}$ by $\tilde{X}_{j}^{m} \equiv X_{j}^{m} /\left(1-\mu_{j}^{(i)} n^{(i)} \cdot X^{m}\right)$ and look for the linear fit coefficients to the map from $\left\{\tilde{X}^{m}\right\}$ to $\left\{x^{m}\right\}$. This order of steps is then repeated until all the parameters converge to the desired precision.

A standard way to check the goodness of fit of the camera model is to do the stereoscopic reconstruction on the calibration point images. This is usually accompanied by a few iterations of adjusting the prescribed mask positions, since in reality the mask can slightly rotate and shift relative to the desired position. At each iteration a stereoscopic reconstruction is performed and followed by rigid body movements of all the calibration points belonging to a single mask position, in such a way as to minimise the difference between the best stereoscopic fits and the actual locations of the calibration points. In Fig. 3.25 I show the results of our calibration using two iterations of mask position adjustments.

In Fig. 3.25a, I plot the histograms of the component-wise differences between the best 

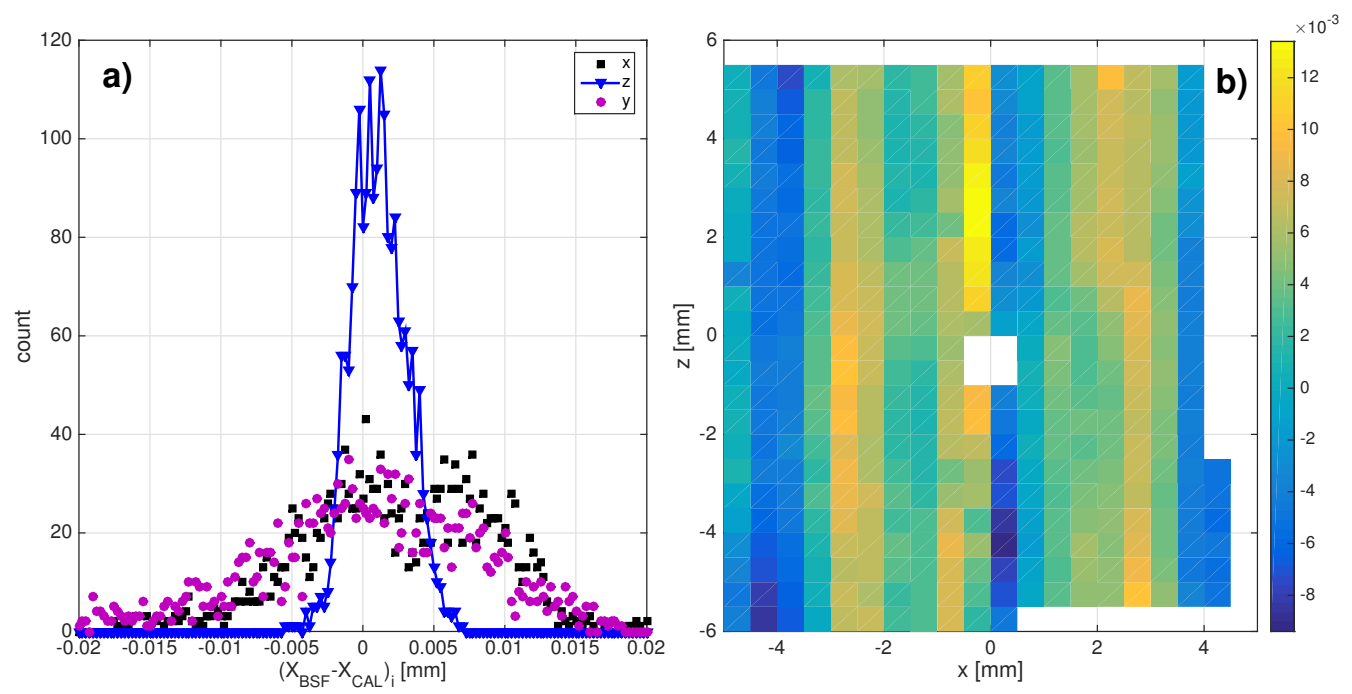

Figure 3.25.: Analysis of the goodness of fit of the uncorrected two-slit model to the mapping of the calibration points. In (a) I show the histograms of component-wise differences between the locations of the actual calibration points and the stereoscopic best fit reconstructed from their images. Ideally the distributions for all components should be similar, however in our case the fit is much better in the vertical direction than in the other two. In (b) I show the $x$-component of the actual point vs. best fit difference as a function of its $x$ and $z$ position, averaged over the $y$ direction. The magnitude of the difference ranges between $-9 \mu \mathrm{m}$ (dark blue) and $12 \mu \mathrm{m}$ (yellow).

stereoscopic fits and the actual positions of the calibration points. As has been described above, a difference of $12 \mu \mathrm{m}$ corresponds to about one pixel difference on the sensors, and is considered large. The distribution of the $z$-component of the difference follows an expected form with a standard deviation of about $2 \mu \mathrm{m}$, whereas the other two component-wise differences have a similarly wide distribution with a standard deviation of roughly $7 \mu \mathrm{m}$. A clue to the cause of this aberrant behaviour is provided by the plot in Fig. 3.25b where I show the $x$-component of the difference between best fit and actual position of calibration points, as a function of the point's $x$ - and $z$ - location (results are averaged over $y$-th component). I see that the difference is largely independent of the vertical position ( $z$-component) and strongly dependent on the $x$-component. This is in fact caused by small imperfections in the thickness of the cylindrical glass shell, which lead to significant changes in the refracted 
angle of the incident light. These imperfections were found to be largely constant in the vertical direction. Therefore, a corrective function must be added to the mapping to account for this distortion effect, that is a function of the horizontal sensor position only and shifts the image again only in the horizontal direction:

$$
x_{\text {corr }}=x+e_{x} f_{\text {corr }}\left(x \cdot e_{x}\right) \quad \text { where } \quad e_{x}=[1,0] \quad \text { and } \quad x=\Pi(X) .
$$

Since no elegant functional form can be expected to fit the distortion profile, the corrective function must be prescribed at each $x$ and determined from a large number of sample points. The set of calibration points provides some initial estimate of the correction profile, but it is not dense enough to supply information everywhere. Therefore, the correction profile is determined iteratively using a set of stereoscopically reconstructed particles in an actual video recording. At each iterative step, the stereoscopically reconstructed particles are mapped back onto the camera sensors using the camera model and the correction function from previous iterative step. A mean difference between $x$-components of the mapped images and actual images at each horizontal ( $x$-) sensor position is calculated and added to the correction function to make the new profile. In Fig. 3.26, I show the final correction functions for all three cameras.

Using the two-slit model coupled with the correction function, I managed to bring the distributions of component-wise differences between best-fit and actual particle images down to a similar level for each component, as shown in Fig. 3.27. However, there remains a narrow region of the measurement volume where the stereoscopic reconstruction accuracy is comparatively low, due to the existence of a discontinuity in the correction function for camera number 1 . The discontinuity is a result of the lines of sight crossing each other before reaching the sensor. 


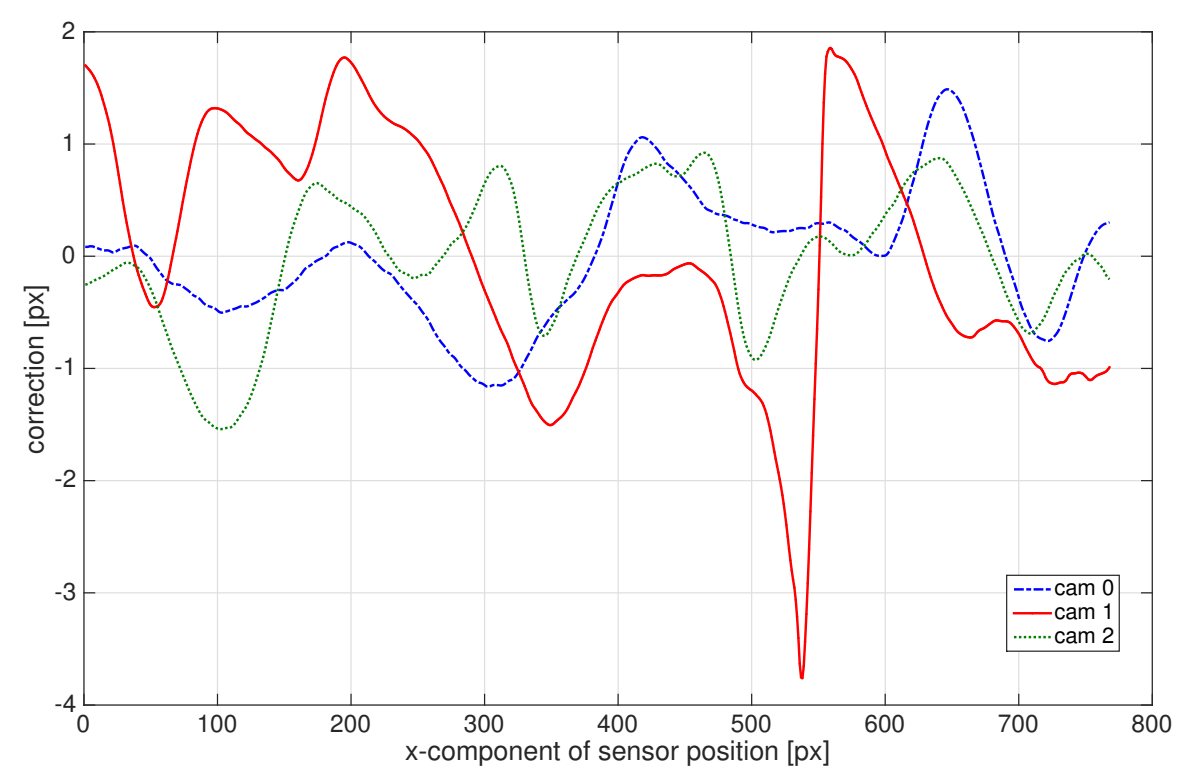

Figure 3.26.: The correction functions $f_{\text {corr }}^{c}$ for the three cameras used, plotted against the horizontal component of the sensor position. The profiles have been obtained from statistics of stereographic reconstruction of a large-density high-speed video of particles travelling through the measurement volume, and subsequently smoothed to remove features under 10 pixel width. The discontinuity of $f_{\text {corr }}^{1}$ at $x=540$ is clearly visible.

\subsection{Tracking in Time}

Once the three-dimensional particle positions are reconstructed, it remains to connect the positions corresponding to individual particles from frame to frame and thus to track the particle positions in time. The procedure I adopted here is not too dissimilar from that adopted by [Ouellette 2006], with only minor modifications.

I proceed sequentially, processing one frame at a time and keeping all active trajectory information stored in memory. Let us denote the position of the particle belonging to the $i$-th trajectory at the $n$-th frame as $X^{i}\left(t_{n}\right)$, and the length of the $i$-th trajectory, that is the number of stored particle locations before the current frame, as $L^{i}$. At each frame, I first perform the stereoscopic reconstruction. Then I go through the list of currently active trajectories and try to extend each one of them to the current frame, i.e. select the point from the set 


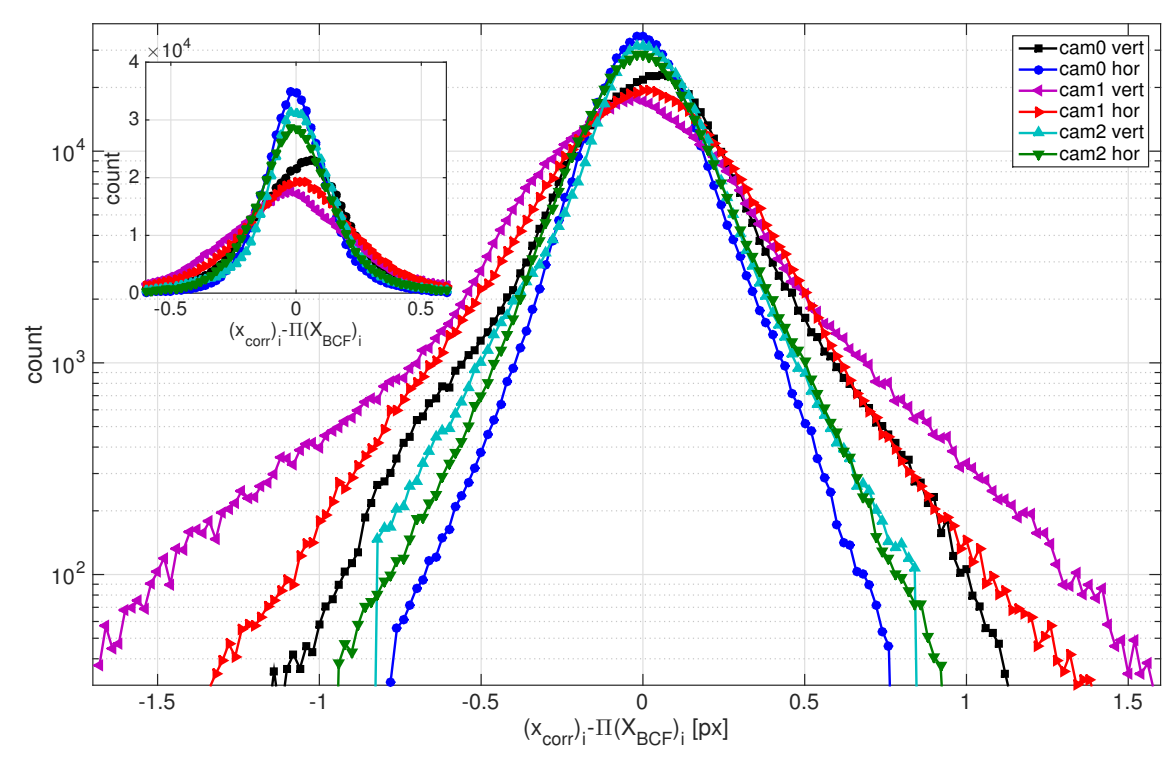

Figure 3.27.: Histograms of the component-wise differences between the projections of the best stereoscopic fit using a two-slit model with a corrective function, and the actual particle images, shown on a semi-logarithmic and linear scales (inset), for a typical experimental run. Use of the corrective function has ensured that the majority of the differences fall below 0.25 pixel.

of reconstructed particle positions that represents the current location of the particle being tracked by the corresponding trajectory. This is done in the following way: for each trajectory, based on the previous particle positions, an estimate $X_{\text {est }}^{i}\left(t_{n+1}\right)$ of the position in the current frame is made and a region $\mathfrak{D}$ of acceptable departure from this estimate is established, in a manner dependent on the trajectory length:

- If $L_{i}=1$, that is the particle has only been seen once before, I set $X_{\text {est }}^{i}\left(t_{n+1}\right)=X^{i}\left(t_{n}\right)$, i.e. the same position as the one last seen. The acceptable departure region can then be written as $\mathfrak{D}=\left\{X|| X_{j}-\left(X_{\text {est }}^{i}\right)_{j}\left(t_{n+1}\right) \mid<V_{j}^{\max }\right\}$ with $V^{\max }=[0.15,0.15,0.8]$ $\mathrm{mm} / \mathrm{frame}$. The reasoning behind this choice of limits is that the difference in the detected positions of a particle between two successive frames is caused by two factors: an instantaneous velocity of the particle, and the noise inherent in the process 
of particle positioning and stereoscopic reconstruction. As shown above, the standard deviation of the positioning and reconstruction process is roughly $3 \mu \mathrm{m}$ and can be neglected relative to the displacements caused by particle motion, which can reach up to hundreds of $\mu \mathrm{m}$. The limits on $V^{\max }$ that I chose were obtained from the velocity statistics of several videos. Since the particles are mainly streaming in the vertical direction, the velocity limit in the vertical direction is several times higher than in the horizontal directions.

- If $L_{i}=2$, I set $X_{\text {est }}^{i}\left(t_{n+1}\right)=2 X^{i}\left(t_{n}\right)-X^{i}\left(t_{n-1}\right)$, which is the linear extrapolation of the last two positions, and $\mathfrak{D}=\left\{X|| X_{j}-\left(X_{\text {est }}^{i}\right)_{j}\left(t_{n+1}\right) \mid<100 \mu m\right\}$.

- If $L_{i}=3$, I set $X_{\text {est }}^{i}\left(t_{n+1}\right)=\frac{4}{3} X^{i}\left(t_{n}\right)+\frac{1}{3} X^{i}\left(t_{n-1}\right)-\frac{2}{3} X^{i}\left(t_{n-2}\right)$, which is the minimumvariance linear extrapolation from the last three positions and $\mathfrak{D}=\left\{X|| X_{j}-\left(X_{\text {est }}^{i}\right)_{j}\left(t_{n+1}\right) \mid<80 \mu m\right\}$.

- If $L_{i} \geq 4$, I set $X_{\text {est }}^{i}\left(t_{n+1}\right)=X^{i}\left(t_{n}\right)+\frac{1}{2} X^{i}\left(t_{n-1}\right)-\frac{1}{2} X^{i}\left(t_{n-3}\right)$, which is the minimumvariance linear extrapolation from the last four positions and $\mathfrak{D}=\left\{X|| X_{j}-\left(X_{\text {est }}^{i}\right)_{j}\left(t_{n+1}\right) \mid<60 \mu m\right\}$.

The reason for the shrinking bounds in the definion of $\mathfrak{D}$ with increasing trajectory length is that with increasing amount of information I can limit the influence of positioning noise on the value of the estimated position, which is the dominant factor in the observed difference between estimated and actual particle position. Given $X_{\text {est }}^{i}\left(t_{n+1}\right)$ and $\mathfrak{D}$, I search the region $\mathfrak{D}$ for any stereoscopically reconstructed particles, and select the one that is closest to $X_{\text {est }}^{i}\left(t_{n+1}\right)$. If there are no reconstructed particles in $\mathfrak{D}$ then the trajectory is terminated, and if it had reached a given threshold on the trajectory length (in our case the threshold was 10 frames), its information is saved into an output file. 
Once all the active trajectories have gone through the extension procedure, I check for possible trajectory collisions, that is, for pairs of trajectories which share the same particle position in the current frame. For each such collision, I terminate the shorter trajectory (and save it if it has passed the length treshold). In case of both trajectories having the same length, I terminate the one for which the current particle position was further away from the estimated position. Finally, all stereoscopically reconstructed particles that have not been assigned to any trajectory are used to initiate new trajectories of length one. With that the processing of the current frame is done and the algorithm moves on to the next frame. After all the video frames are processed, the algorithm checks all the currently active trajectories and saves the ones meeting the length criterion. 


\section{CHAPTER 4 \\ Experimental Results}

In this chapter, I present the results of experiments conducted at sub-atmospheric pressure conditions. In section 4.1, I first characterise the experimental data. I then present the statistical properties of the particle dynamics in section 4.2. In section 4.3, I demonstrate the occurrence of particle aggregation and clustering on small scales. Finally, in section 4.4, I consider the influence of the global system properties, such as the mean particle number density (equivalently, the background mass loading or volumetric filling factor).

\subsection{Data Characterisation}

I analyse four particle-tracking data sets (DS) corresponding to different steady-state pressure conditions. All of the DS involve steel particles in the range 15-65 $\mu \mathrm{m}$. The measurement volume, $v_{\text {meas }}$ as defined in section 3.3, is fixed at $\sim 5.5$ pipe diameters downstream from the particle seeding platform/gas inlet. Figure 4.1 places these DS in the context of the drag regime to which they belong. The figure shows the mean free path (and multiples thereof) for pressures between 0-20 mbar. The y-axis shows the length scale and references particle sizes 
that will fall in the various pressure-dependent regimes. The four vertical lines correspond to the pressures of the different DS and the length of the line represents the polydispersity of the particle population. All values to the right of the solid black line are $K n<1$, where a continuum description of the gas is generally considered valid. Also shown is the regime in which 'free-slip' conditions apply, where detailed theoretical models generally also approach the gas as a continuum, with some special consideration for the boundary conditions at particle surfaces. The dashed grey line shows the mathematical Stokes-Epstein transition, which is commonly used as an approximate dividing point between continuum and freemolecular flow conditions. While the Epstein drag law clearly applies for the latter, the effect of drag on solid surfaces in the transition region for $0.1<K n<10$ is a very open field of research. Two of the DS (DS1, DS2) are at pressures corresponding to $K n \sim 1$ and I compare them to additional DS with greater pressure (DS3, DS4) and $K n<1$. Each DS consists of $\sim 10$ recordings ${ }^{1}$ that are each 6 -seconds.

\footnotetext{
${ }^{1}$ With a few more (less) movies depending on how sparse (dense) the particle seeding is.
} 


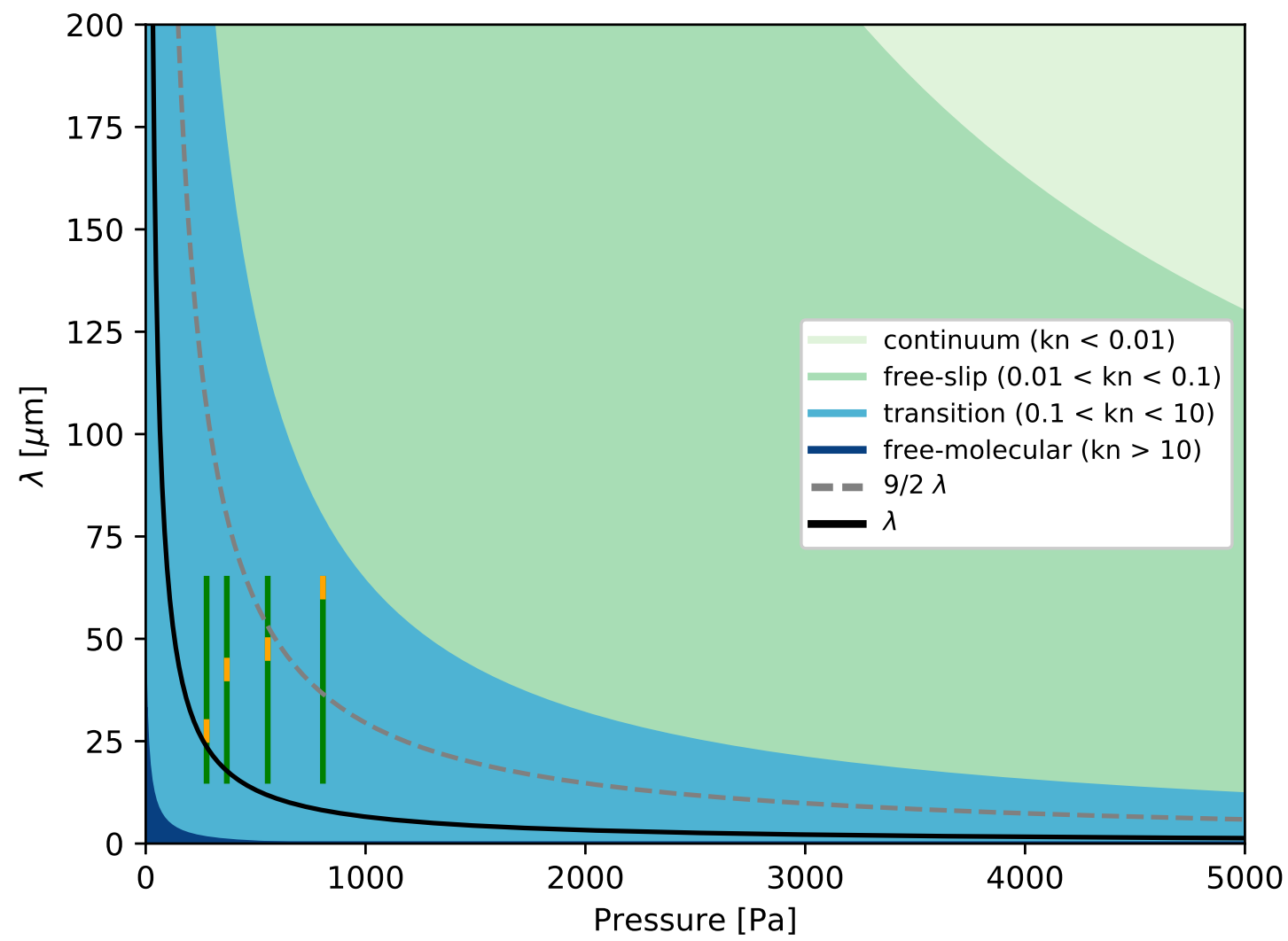

Figure 4.1.: Gradations in drag regime, around $K n=1$. Vertical green lines represent the four data sets; their height represents the maximum distribution width of particle sizes and the horizontal thickness of the green lines represents the typical offset in pressure ( . $1 \mathrm{mbar}$ ) between the sensors at the bottom, middle, and top of the container $(\mathrm{H} 1, \mathrm{H} 2$, and $\mathrm{H} 2$ in figure 3.1, sensor offset visible in the inset of figure 3.6). Orange bars represent the particles sizes constrained by the mean z-direction velocity compared to the expected terminal velocity of particles belonging to either drag regime represented by the $9 / 2 \lambda$ division. 
The range of particle sizes indicated in 4.1 can be narrowed for each DS by considering the mean $z$-direction velocity of the particles. First, consider the gas velocity in the region of the flow corresponding to the location of the particle-tracking measurements. Figure 4.2 shows a closeup of the central two centimeters of the flow profile with the mean of the measured values shown as dots and the error bars are from the standard deviation of them. The solid line is the parabolic fit. The error contributes little to the variation in velocity, and for reasons explained in the previous chapter, I assume the profile is the same for all pressures and I expect for the size of the errors to only decrease for lower pressure (lower $R e$ ). The flow velocity does not varry greatly from its maximum of $1.4 \mathrm{~m} / \mathrm{s}$ over the central centimeter. This is quantified by calculating the difference in velocity with respect to the maximum, as shown in the bottom panel of 4.2 . The figure shows that the variation in flow velocity is typically no more than $\sim 1 \%$ in the central centimeter or $\sim 5 \%$ in the central two $\mathrm{cm}$. 

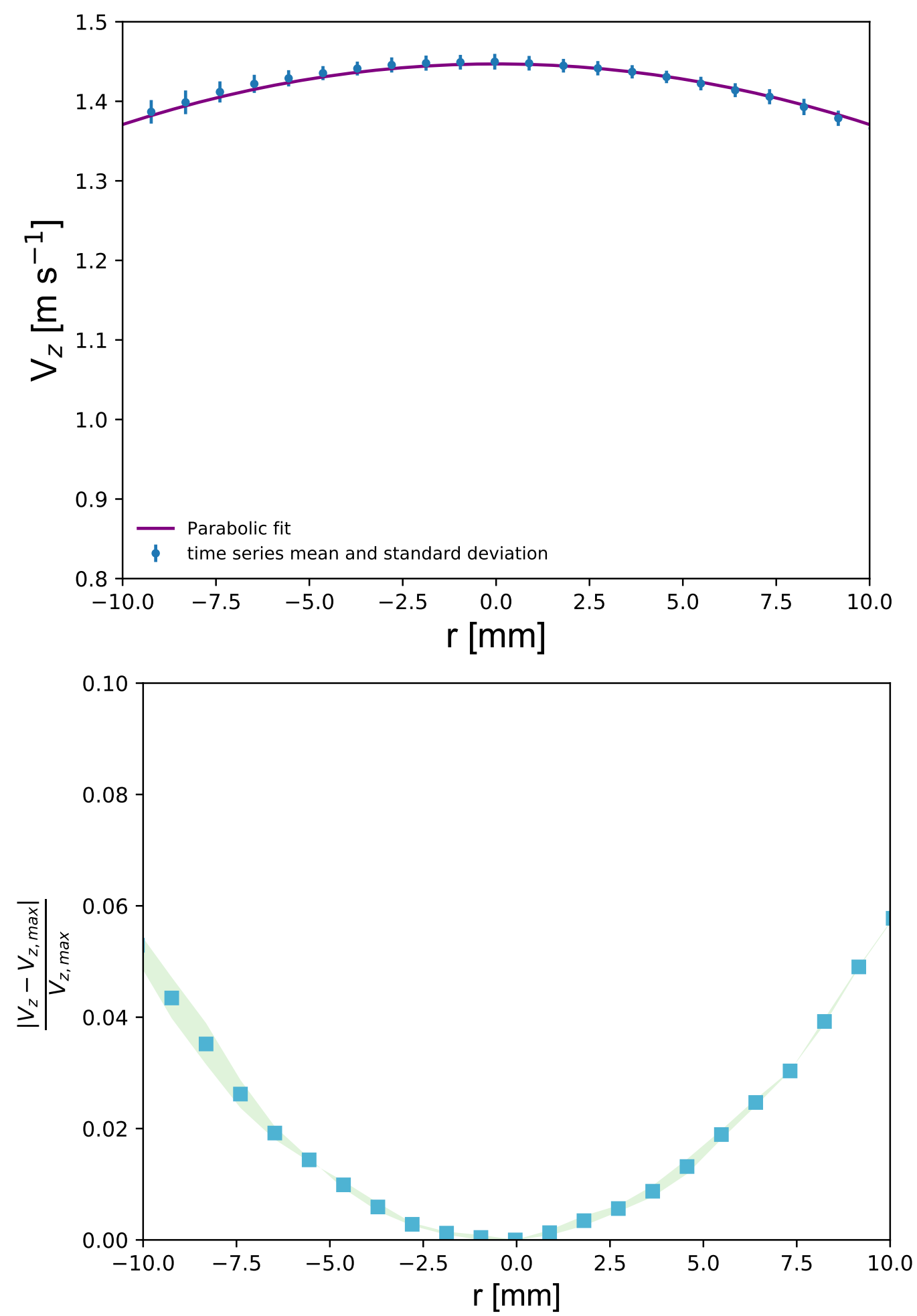

Figure 4.2.: Top: central two centimeters of the mean flow profile measured with PIV. The full profile is shown in figure 3.15. Dots represent the mean profile and error bars represent the standard deviation of the time-series PIV data. The purple line is the fit to the mean profile. The $\mathrm{V}_{z}$ axis is on the same scale as that of the range measured data points. Bottom: change in velocity over the central region. Markers correspond to the mean velocity and the colored region to the range indicated by the error bars. 
The expected vertical velocity of the particles is just the difference between the gas velocity and the terminal velocity of a sphere, according to the pressure-dependent drag law and of a given diameter. In Figure 4.3, I show the measured mean vertical velocity corresponding to the pressure value of each dataset. I overplot curves of constant particle size: from top to bottom, they are for 25,45 , and $65 \mu \mathrm{m}$. The lower curve becomes constant at 6 mbar and above, representing the Stokes-Epstein transition, where the drag law, and therefore the terminal velocity of the particle, is not pressure-dependent. It is apparent from the placement of the measured data points that there is a size selection effect with pressure, despire the fact that the same population of particles were inside the container. The lowest pressure gas is selecting the smallest particles and the highest pressure gas is selecting the largest particles, etc. This is to be expected, since only particles meeting the criterion $u_{t}<u_{g a s}$ will enter the gas stream, and since $u_{t}$ is pressure dependent across these DS, the particle size varies as well. With this information, I confirm that DS1 and DS2 have $K n \geq 1$, since the selected particle population tends towards the smaller side of the distribution. The other two DS tend to have particles on the larger end of the distribution and are therefore in the continuum gas region. Moreover, since the mean vertical velocities of all of the DS fall within the expected curves of particle velocity, the assumption that $u_{g}=1.4$ holds for all DS cannot be too flawed. Even though the particle size likely varies between the DS, it affects only the mean velocity since $d_{p}<<\phi^{-3 / 2}$ and so a meaningful comparison can still be made across DS. Perhaps the effects of polydispersity, which is an aspect that I don't visit in this investigation, can rightfully be ignored, since it appears that the flow conditions naturally favor specific size regimes $^{2}$.

\footnotetext{
${ }^{2}$ Note that the particle sizes reported here are lower limits, since the drag regime transition is approximate. See further comment in the Discussion and perspectives section.
} 


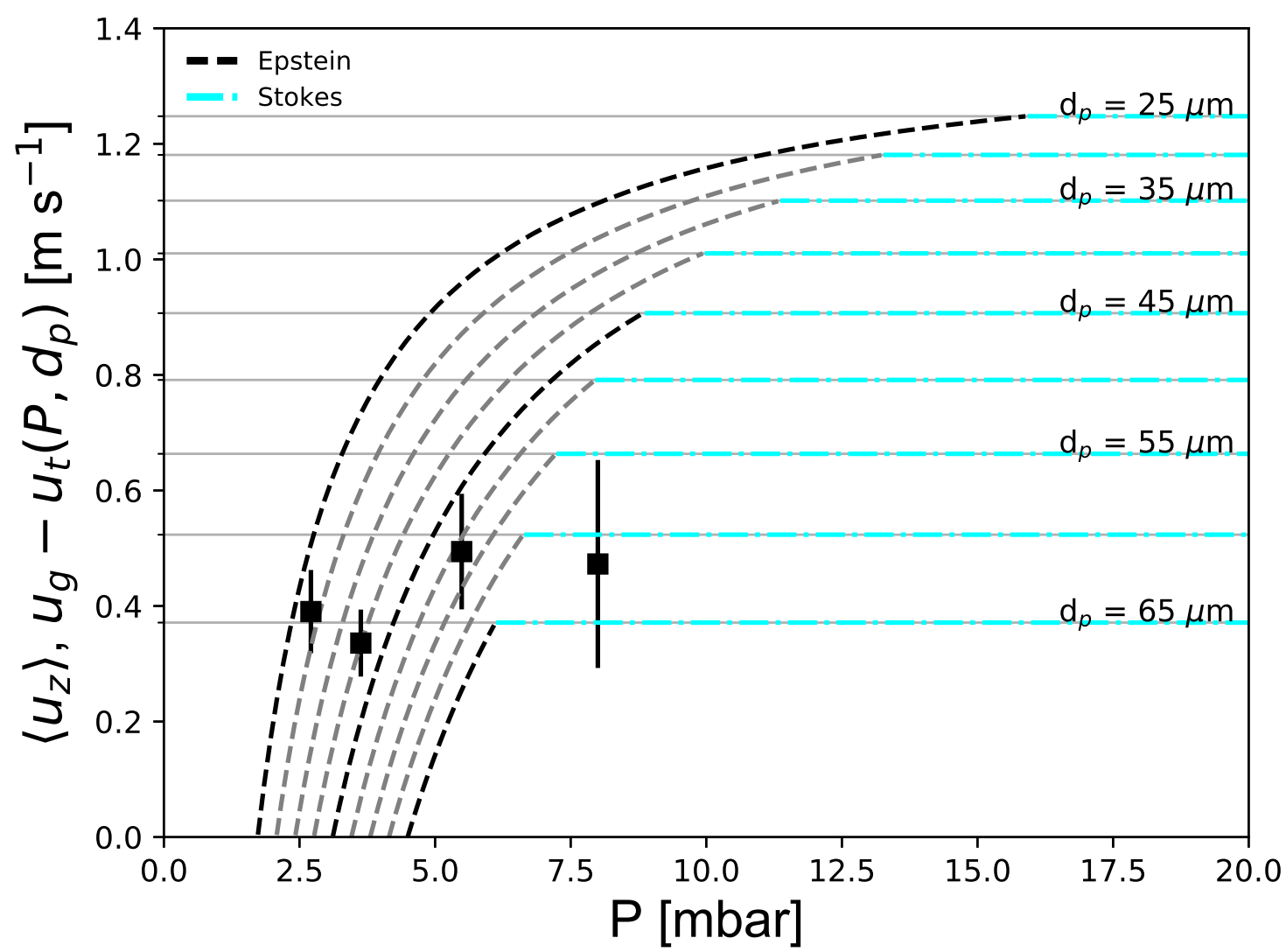

Figure 4.3.: Squares are the mean measured particle velocities for each dataset. Curves of expected particle velocity in the Epstein (dashed; grey and black) and Stokes (dasheddotted; cyan) drag regimes. Minor axis lines (solid, grey) are extended from the expected velocity assuming that the drag is pressure independent, as in the Stokes drag case. 
Table 4.1.: Conditions and properties of data sets 1-4. Values from left to right are Pressure in mbar, mean mass loading, volumetric filling factor, number of particles per frame, the container-scale and particle-scale Reynolds' numbers, the friction time in seconds (with the drag law assumed indicated by either St for Stokes or Ep for Epstein), momentum diffusion time in seconds and the total number of trajectories analysed.

\begin{tabular}{cccccccccc}
\hline \hline Data Set & $\mathrm{P}$ & $n$ & $\phi$ & $\varepsilon$ & Re $_{\text {container }}$ & $R e_{\text {particle }}$ & $T_{f}$ & $t_{d}$ & $N_{\text {traj }}$ \\
\hline DS1 & 2.71 & $1 \pm 1$ & $5.9 \mathrm{e}-09$ & 0.015 & 15 & 0.002 & $0.091_{E p}$ & 0.0004 & 5758 \\
DS2 & 3.63 & $13 \pm 4$ & $4.916 \mathrm{e}-07$ & 0.92 & 20 & 0.003 & $0.12_{E p}$ & 0.014 & 65470 \\
DS3 & 8.0 & $21 \pm 10$ & $1.857 \mathrm{e}-06$ & 1.57 & 45 & 0.018 & $0.10_{S t}$ & 0.079 & 172615 \\
DS 4 & 5 & $2 \pm 8.29$ & $2.48 \mathrm{e}-07$ & 0.31 & 21 & 0.0097 & $0.089_{E p}$ & 0.011 & 99816 \\
\hline
\end{tabular}

Table 4.1 summarizes the properties of the four DS. For any given single experiment (movie) in a DS, the particle number density fluctuates about a constant mean. I estimate the typical number of particles in the volume, $n$, by tallying the number of particles in each time frame and taking the mean for the whole movie. The value of $n$ can vary for different iterations of the experiment. The table presents the mean $n$ of all experiments in each DS, plus or minus the standard deviation of $n$ for the entire DS. By varying P and $n$, The parameter $\varepsilon$ also varies. To calculate $\varepsilon=n m_{p}\left(d_{p}, \rho_{p}\right) / \rho g$, I determined the total mass of the solids by multiplying $n$ by the mass of the individual particles with the density of steel, $\rho_{p}=8.9$ $\mathrm{gm} \mathrm{cm}^{-3}$. I adopted particle diameters of $d_{p}=25,45,45,65 \mu \mathrm{m}$ for DS1, DS2, DS3, DS4, respectively. I have determined $\rho_{g}$ from the pressure (see 4.1) and temperature (see 3.1.3) calibrations, assuming an ideal gas, with molar gas constant $\mathrm{R}=8.314 \mathrm{~m}^{3} \mathrm{~Pa} \mathrm{~K}^{-1} \mathrm{~mol}^{-1}$, and molar mass of dry air $\mathrm{M}=0.02891 \mathrm{~kg} \mathrm{~mol}^{-1}$. Similarly, $\phi=n V_{p}\left(d_{p}\right) / V_{\text {meas }}$ is estimated by counting the particles per frame, taking their mean for an entire movie and dividing by $v_{\text {meas }}$. The table values of $\phi$ and $\varepsilon$ are the median of all movies in the DS. The $R e_{p}$ was calculated using the largest component of the root mean square velocity as the characteristic velocity and $R e_{\text {container }}$ using the mean of the global flow profile, $0.95 \mathrm{~m} \mathrm{~s}^{-1}$. 


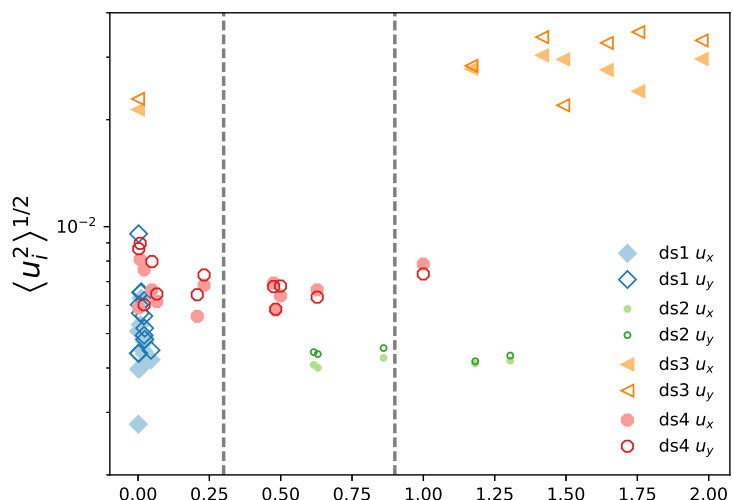

$\langle\varepsilon\rangle$

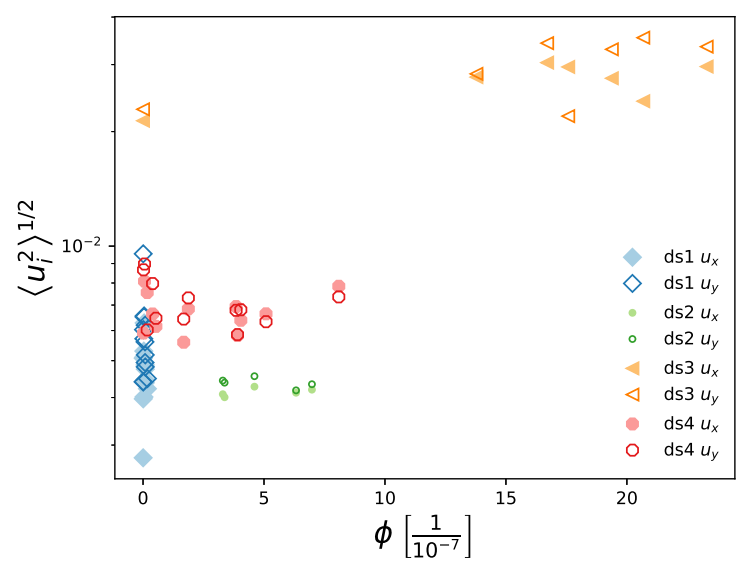

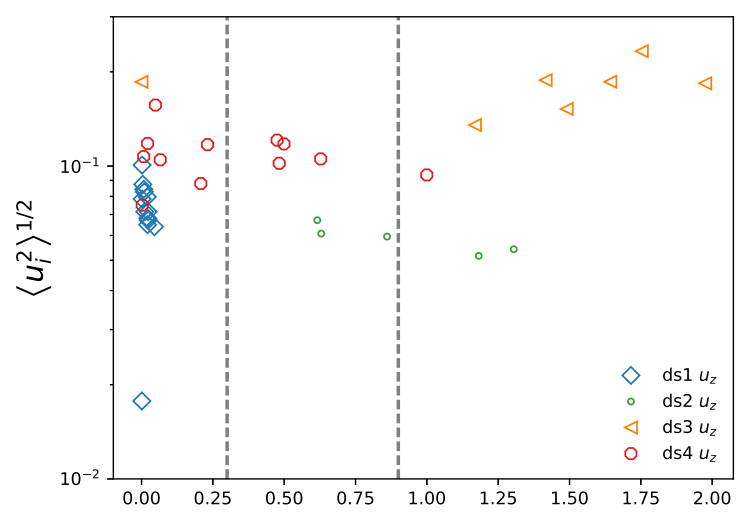

$\langle\varepsilon\rangle$

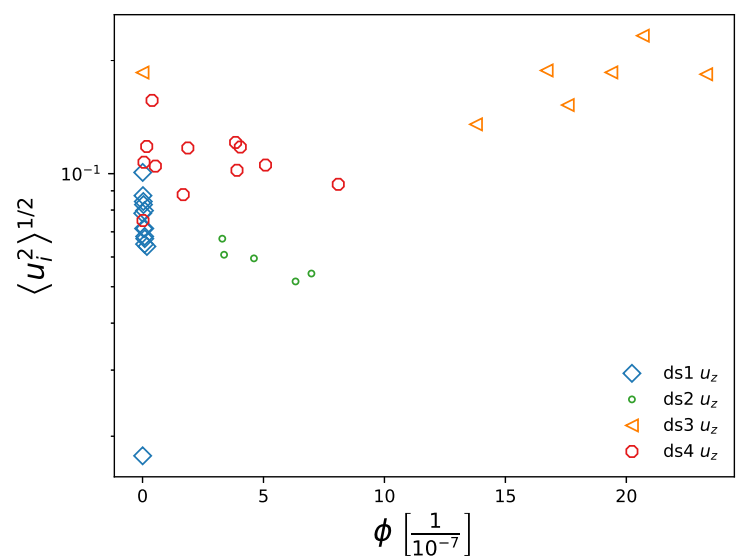

Figure 4.4.: The root mean square velocity of all experiments shown as a fuction of either $\varepsilon$ (top) or $\phi$ (bottom). In all panels, DS1 is represented by blue diamonds, DS2 by green points, DS3 by orange left arrows, DS4 by red octagons. The left column shows the two horizontal components, with the filled symbols corresponding to the x-component and unfilled to the y-component. The right column is the vertical component. Dashed vertical lines in the $\varepsilon$ vs. rms velocity show where a cut in mass loading has been made for part of the analysis. 


\subsection{Global statistical properties}

For spheres at large interparticle distance, one expects their individual settling velocities to remain nearly constant, as is the case for isolated spheres traveling at their terminal velocity. The friction time of all of the particles is short enough that the particles are already coupled to the gas well before reaching the measurement volume; at the typical gas velocity in this system, one expects the particles to reach their terminal velocity by the time they have traveled just a few centimeters above the location at which they first become fluidised. One expects for the horizontal velocity components to be nearly zero, with perhaps a small imprint of perturbations that occur when the particles are initially entrained in the flow. In the event of an unstable particle-fluid mixture, theoretical predictions (see Lambrechts et al. (2016)) indicate that exponentially growing fluctuations of the particle-velocity field should saturate within a timescale of 6 friction times or less, corresponding to a height of no more than $30 \mathrm{~cm}$, which is also well below the camera observation height $(55-60 \mathrm{~cm})$. In figure 4.5 I show an analytical estimate of the hight evolution of of the particles in each DS, using the particle sizes suggested by figure 4.3 and their corresponding friction times. 


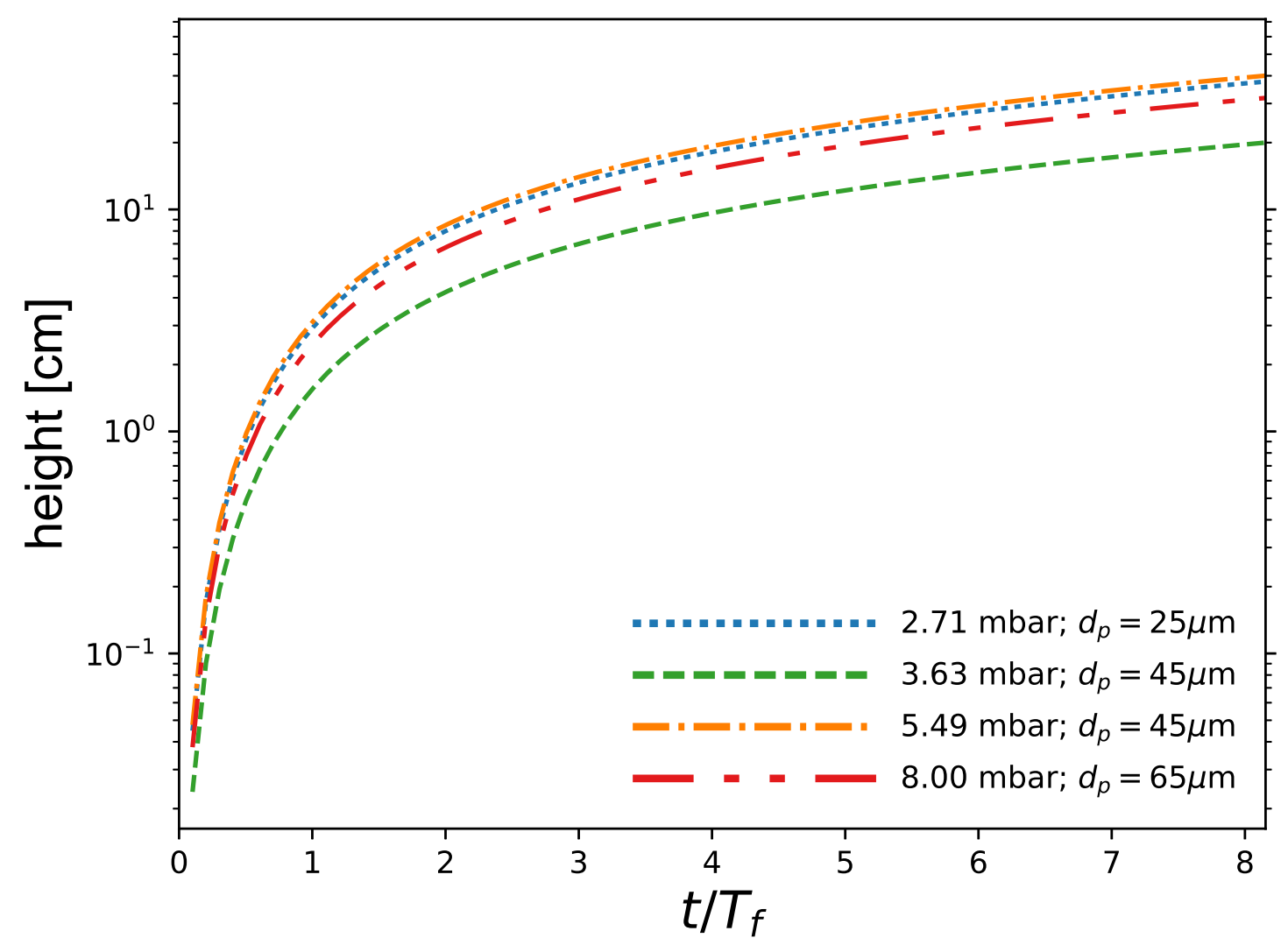

Figure 4.5.: Analytical calculation of the distance over which the particles couple to the gas in an e-folding time, extended over eight such time scales to demonstrate at which height the predicted instability should be fully developed. The friction times used are listed in table 4.1 
The root mean square (rms) velocity of all the experiments versus either the mean value of $\phi$ or $\varepsilon$ are shown in figure 4.4. The vertical velocity component's rms velocity is in general two orders of magnitude greater than that of the horizontal components. In all cases, the rms velocity remains nearly constant for the entire DS, even though the individual experiments comprising the dataset can span a range of $\phi$. The parameters $n, \phi$, and $\varepsilon$ are nearly inter-changeable quantities, since the second two of these quantities depend directly upon the first and are just offset by a multiplicative factor depending upon the pressure of the DS. For the $K n<1$ data, $\phi$ is relatively high compared to the other data sets, but $\varepsilon$ less so because the higher gas density of DS3 and DS4 in the denominator of the mass loading drives it down. DS 4 spans the widest range in $n$ (and consequently $\phi$ or $\varepsilon$ ). DS1 contains very sparsely seeded flows and the typical rms velocities show a wide scatter. There is a downward trend in z-direction rms velocity with increasing $n$. 

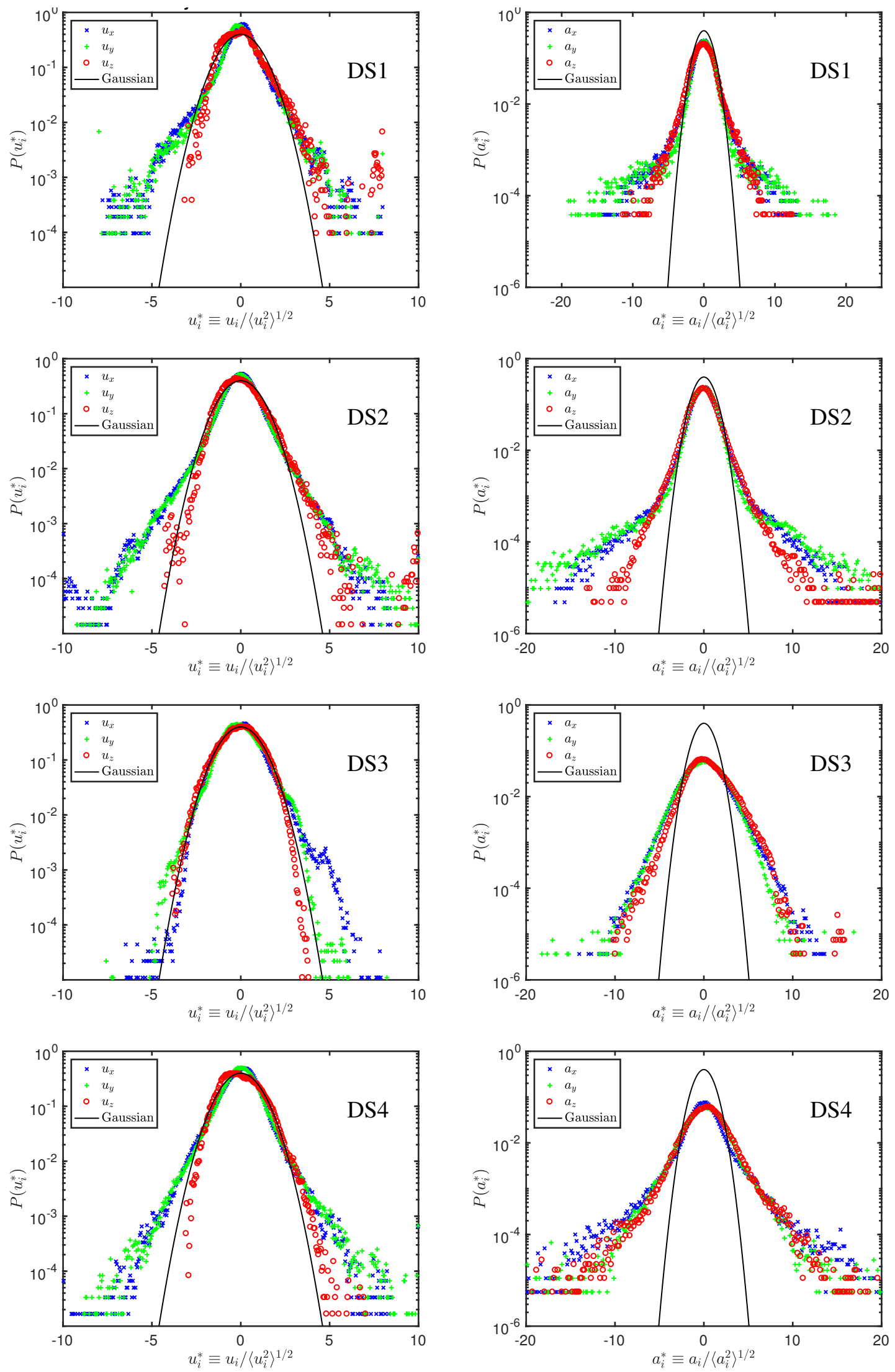

Figure 4.6.: Velocity (left column) and acceleration (right column) PDFs for DS1-4 (top to bottom). Blue $\mathrm{x}$ 's, green plus marks, and red circles correspond to the $\mathrm{x}, \mathrm{y}$, and $\mathrm{z}$ components, respectively. All PDFs are normalised by their rms value. Gaussian overplotted for comparison. 
Figure 4.6 shows the probability distribution functions (PDFs) of the velocity and acceleration, decomposed into components, of the individual particle trajectories for all DS. The instantaneous velocities and accelerations are normalised by their rms values. An equivalent gaussian distribution is overplotted for comparison. The PDFs indicate that the velocity can fluctuate by a factor of up to 10 times the rms velocity. This is particularly true of the horizontal velocity components, whereas the vertical velocity component tends to have a narrower, however more skewed distribution ${ }^{3}$.

The velocity PDFs of DS3 stand out, since all three components are closer to a Gaussian distribution. Some additional surprising features in the PDFs are the population of high z-velocities in DS1 and DS2 and the apparently broader tails in the acceleration PDFs of DS3 and DS4. All of these changing features could result for different reasons; since DS1 and DS2 show similarities and are at lower pressures, while DS3 and DS4 also show similarities and are at higher pressures, some of these features may be related to the different $K n$ of the DS. In section 4.4 I consider the possibility that the variation of $n$ within the DSs also affects the shape of the distribution and its defining statistics.

To gain an overview of how particles move with respect to one another, one can study the difference in velocity between all coexisting particle pairs, conditioned upon the distance between them. The mean square of this value helps to summarise how the system behaves as a function of scale. To perform this calculation one assumes that two covariate quantities are the difference in velocity between two points $\mathbf{x}+\mathbf{r}$ and $\mathbf{x}$ :

$$
D_{i j}(\mathbf{r}, \mathbf{x}, t)=\left\langle\left[U_{i}(\mathbf{x}+\mathbf{r}, t)-U_{i}(\mathbf{x}, t)\right]\left[U_{j}(\mathbf{x}+\mathbf{r}, t)-U_{j}(\mathbf{x}, t)\right]\right\rangle
$$

Where $i$ and $j$ are either parallel to or perpendicular to the separation vector between the two particles. The components along the separation vector, $l$ are those of interest for particle-

\footnotetext{
${ }^{3}$ Although note that the rms is much smaller for the $\mathrm{x}$ and $\mathrm{y}$ directions, so the normalisation may serve to amplify the apparent fluctuations.
} 
particle relative velocities $D_{i, j}$, with $i=j=l . D_{l l}$, which is referred to as Eulerian secondorder longitudinal velocity structure function, measures the amplitude of the particle relative velocity at different separations. Figure 4.7 shows $D_{l l}$ for all DS. While DS2-DS4 show a slow increase with distance (until the limits of the measurement volume are reached around $1 \mathrm{~cm}$ ), DS1 does not, possibly indicating a lack of correlation between the velocities of the sparsely seeded particles. One might expect for $D_{l l}$ to be dominated by the z-component rms velocity, and it is true that the placement on the y-axis of the 4 curves is roughly in agreement with the way that rms velocity increases with increasing gas pressure, as seen in figure 4.4. Consider that $\left\langle\left[u_{l}(\mathbf{x}+\mathbf{r})-u_{l}(\mathbf{x})\right]^{2}\right\rangle=\left\langle u_{l}(\mathbf{x}+\mathbf{r})^{2}\right\rangle+\left\langle u_{l}(\mathbf{x})^{2}\right\rangle-2\left\langle u_{l}(\mathbf{x}+\mathbf{r}) u_{l}(\mathbf{x})\right\rangle$, and one assumes that as $r \rightarrow \infty$, the velocities at positions $\mathbf{x}$ and $\mathbf{x}+\mathbf{r}$ are independent. Therefore, the last term $\rightarrow 0$. Assuming homogeneity, the longitudinal projection of the velocity will be $1 / 3$ of the magnitude of the velocity, and therefore as $r \rightarrow \infty, D_{l l} \rightarrow 2 / 3\left(u_{r m s, x}^{2}+u_{r m s, y}^{2}+u_{r m s, z}^{2}\right)$.

This expression corresponds to the numerical values, for DS 1-4, respectively,

$$
\begin{aligned}
2 / 3\left[\left(2.29 \times 10^{-5}\right)^{2}+\left(2.87 \times 10^{-5}\right)^{2}+(0.0054)^{2}\right] & =0.0036 \\
2 / 3\left[\left(1.78 \times 10^{-5}\right)^{2}+\left(1.90 \times 10^{-5}\right)^{2}+(0.0034)^{2}\right] & =0.0023 \\
2 / 3\left[\left(7.69 \times 10^{-4}\right)^{2}+\left(9.4 \times 10^{-4}\right)^{2}+(0.036)^{2}\right] & =0.025 \\
2 / 3\left[\left(5.067 \times 10^{-5}\right)^{2}+\left(4.66 \times 10^{-5}\right)^{2}+(0.019)^{2}\right] & =0.013
\end{aligned}
$$

To guide the reader's eye in figure 4.7, there are dashed lines corresponding to these values. Apart from at very small separations, $D_{l l}$ is nearly constant at this expectation value for all radii. DS2 reaches the expectation value for a homogeneous flow for particles with separation around 4 millimeters, but $D_{l l}$ does not plateau, and rather continues to incerase. Both DS3 an DS4 approach the expectation value at radii close to the size measurement volume and it is therefore unknown whether or at which radius they will plateau.

One can ask whether the gradual rise in slope of $D_{l l}$ for DS2-DS4 is due to the variation in 
gas velocity profile. However, I showed in figure 4.2 that the maximum variation should be around $\sim 1-5 \mathrm{~cm} \mathrm{~s}^{-1}$. The contribution to $D_{l l}$ from the variation in flow profile is overplotted in purple in figure 4.7. From this, one sees that neither the slope nor the magnitude of $D_{l l}$ can come directly from the parabolic global gas flow profile.

The rather low $D_{l l}$ at small $r$ and the gradual and continuous rise of three of the DS hints that the particle velocities are similarly correlated over a range of scales and that perhaps the largest scale is outside of the measurement volume. The flatness of $D_{l l}$ for DS1 serves as a contrast; since this DS has much lower $\phi$, it would seem that the particles can be considered as isolated. Because DS1 contains few statistics and there are not coordinated motions of the particles, I largely exclude this data set in the following analysis of the collective particle dynamics and clustering. The other three DS rather have the potential to exhibit collective particle behavior; the details of which are better revealed in the analysis of the individual velocity components presented in the next section.

\subsection{Particle dynamics on local subscales}

To investigate how particles may exhibit collective behaviour on scales smaller than the measurement volume, and to accommodate the fact that the z-direction velocity is the dominant component, it is necessary to adopt a geometry that allows for the particle-pair relative velocity statistics to be explored independently in the horizontal and vertical directions, with respect to the position of any given particle in the measurement volume at any given time. It is therefore natural to consider the vertical-direction velocity statistics as a function of cylindrical radial separation, $r=\sqrt{x^{2}+y^{2}}$, and vertical separation $z$, conditional on the closeness of the particles to one another. Figures 4.8 and 4.9 show the analysis under this geometric construction for DS2, DS3, and DS4. Shown is the relative particle-pair z-velocity, 


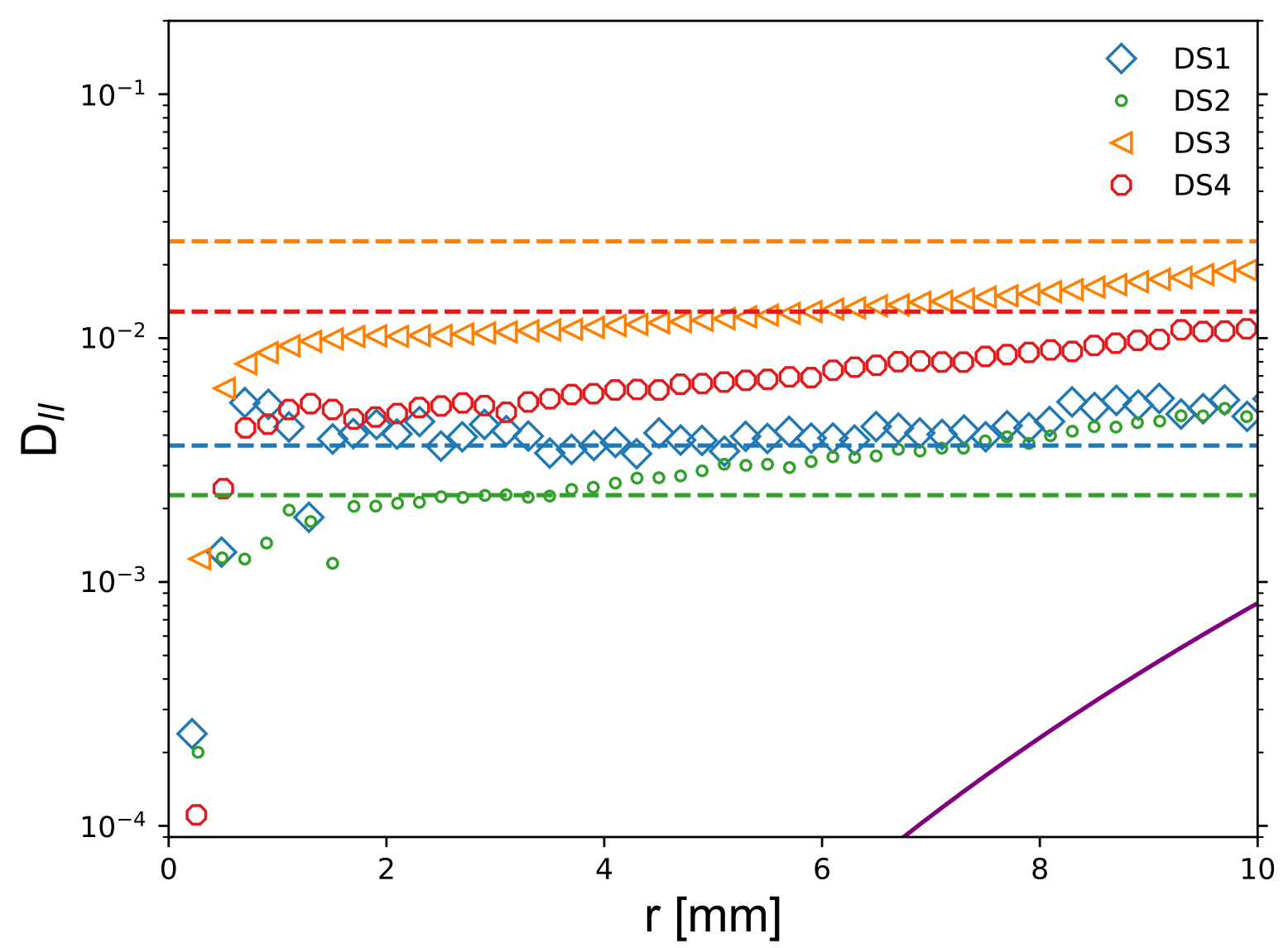

Figure 4.7.: Eulerian second-order longitudinal structure functions for all DS. Coloring and symbols as for figure 4.4 .

$$
\delta u_{i}(\boldsymbol{\delta} r, \delta z) \equiv u_{i}(\mathbf{r}+\delta \mathbf{r}, z+\delta z)-u_{i}(\mathbf{r}, z)
$$

In figure 4.8, the region around a given particle is confined by $1 \mathrm{~mm}$ in either the $r$ or $z$ direction. The component-by-component relative velocities, $\delta u_{x}, \delta u_{y}$, and $\delta u_{z}$ are plotted together on a single set of axes. One notices a tendency for particles to aggregate towards one another that increases sharply for increasingly small separations, particularly less than 4 $\mathrm{mm}$. This figure also demonstrates that the effect can primarily be seen in the vertical, and not the horizontal direction. 
Figure 4.9 shows, again for DS2, DS3 and DS4, $\delta u_{z}$, where conditioning upon radial and vertical separation is the same as for figure 4.8 , except it is not limited to separations less than 1 millimeter. Considering the relative velocities over the extent of the measurement volume, $\delta u_{z}$ is independent of $r$, but takes increasingly large negative values, i.e. particles are approaching one another more rapidly, for smaller vertical separations. It is mysterious why the relative velocities don't arrive at zero for large separations. There is also a directional asymmetry, and it appears that the particles are actually catching up to one another. The results shown in figures 4.8 and 4.9 suggest that the gas-particle mixture is unstable to disturbances of finite non-zero values of wavenumbers $k_{z}$ and that $k_{x}$ and $k_{y}$ are either zero, or else smaller than the size $1 / v_{\text {meas }}$. The noted features are true for each of the DS, despite the different normalisation of the curves and the degree of spread in the curves corresponding to different radial separations ${ }^{4}$.

\footnotetext{
${ }^{4}$ These differences can presumably be understood in terms of the differences between data sets, similarly as to the PDFs. For example, the broad range of $n$ in ds 4 may contribute to the noise in this calculation
} 

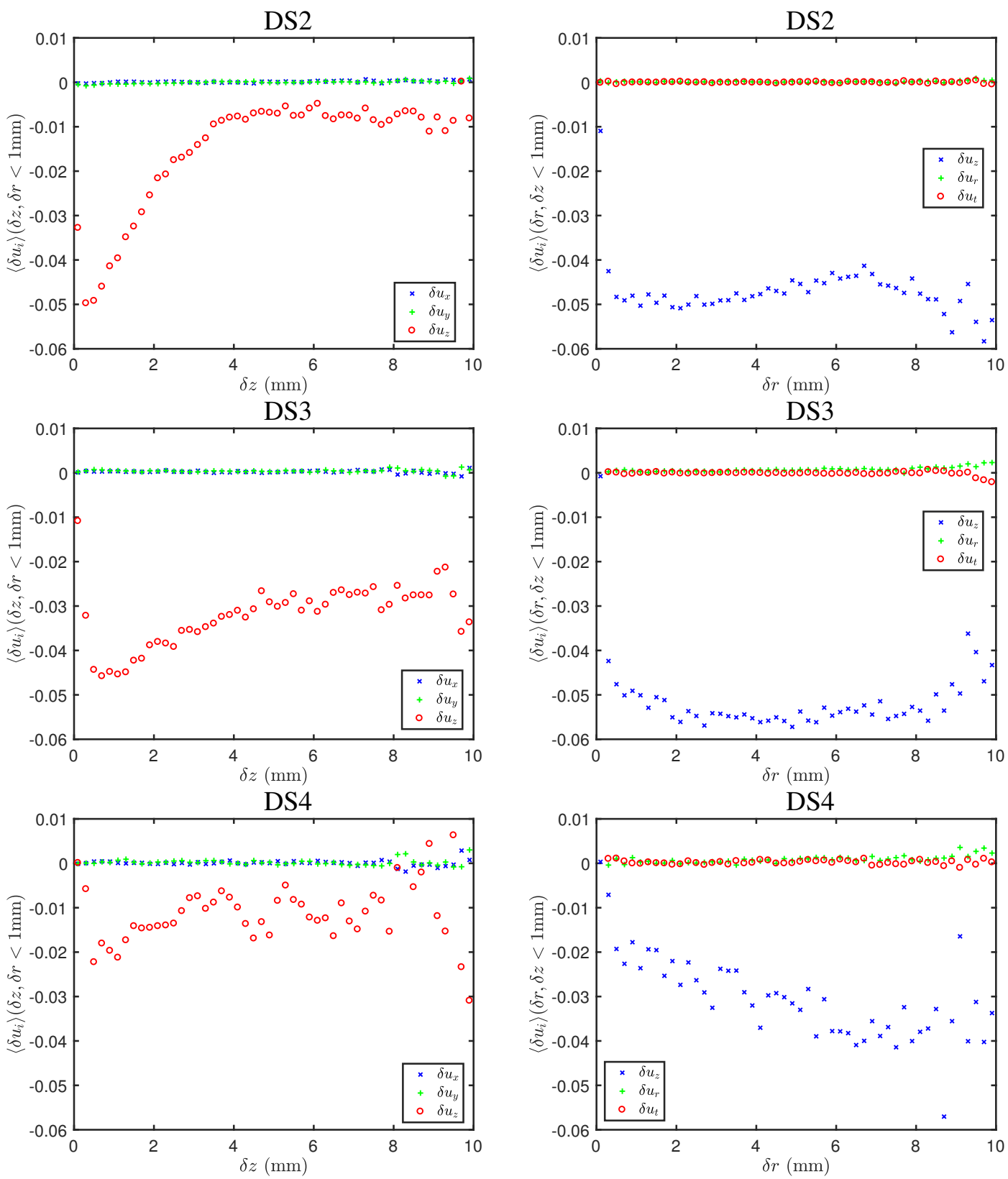

Figure 4.8.: Mean relative particle-pair velocity of each component conditional on cylindrical radius (left) or vertical separation (right) less than $1 \mathrm{~mm}$ for DS2, DS3, and DS4, top, middle, bottom, respectively. 

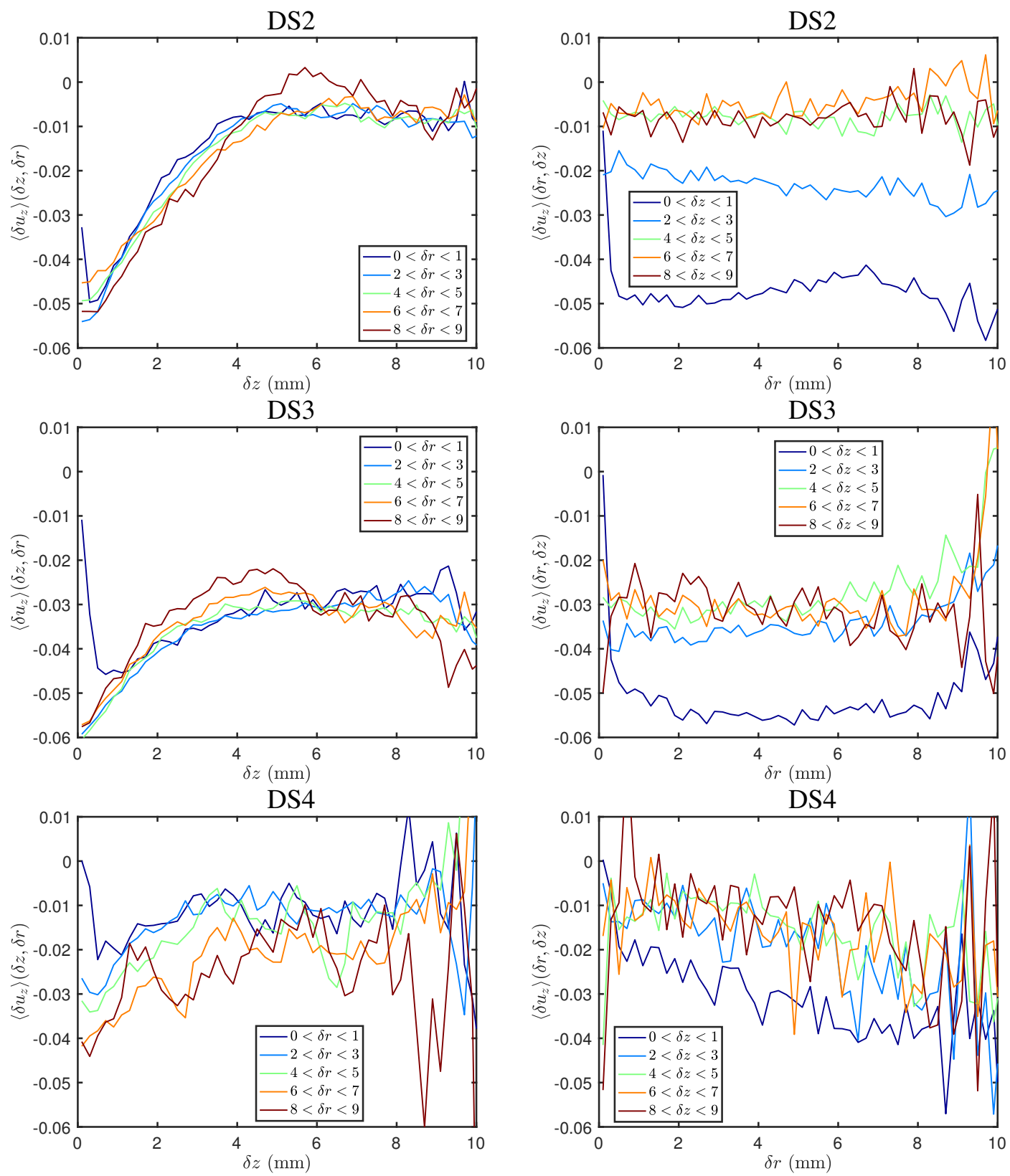

Figure 4.9.: Relative particle-pair z-velocity $\delta u_{z}$, in units of $\mathrm{ms}^{-1}$ for DS2, DS3 and DS4, top, middle and bottom, respectively. Left (right): $\delta u_{z}$ conditioned on cylindrical radius (vertical separation), as a function of vertical separation (cylindrical radius). 
An additional measure of collective particle behaviour on small scales is the temporal variation in local number density, at a fixed location. As already seen in figure 4.8, one expects this variation to occur primarily in the $z$-direction and for there to be little change in the horizontal directions. To explore this metric, I divide the measurement volume into $1 \mathrm{~mm}$-thick cylindrical slices and compute the correlation function of particle number within each slice at times $t$ and time $t+\tau$,

$$
C_{n n}(\tau, z) \equiv \frac{\left\langle n^{\prime}(t, z) n^{\prime}(t+\tau, z)\right\rangle}{\sigma_{n}^{2}(z)} .
$$

Figures $4.10-4.12$ show, in the top panels, the unconditional autocorrelation function at the center of the measurement volume. For comparison, the autocorrelation function is computed for instances where the number of particles is either half as many (dilute case) or 1.5 times as much (dense case) as the mean particle number in the central slice, $\bar{n}$. The comparison shows that the correlation time is shorter for the dense case, revealing that when there are more particles than average in the defined region, they are arranged in a more compact configuration. 

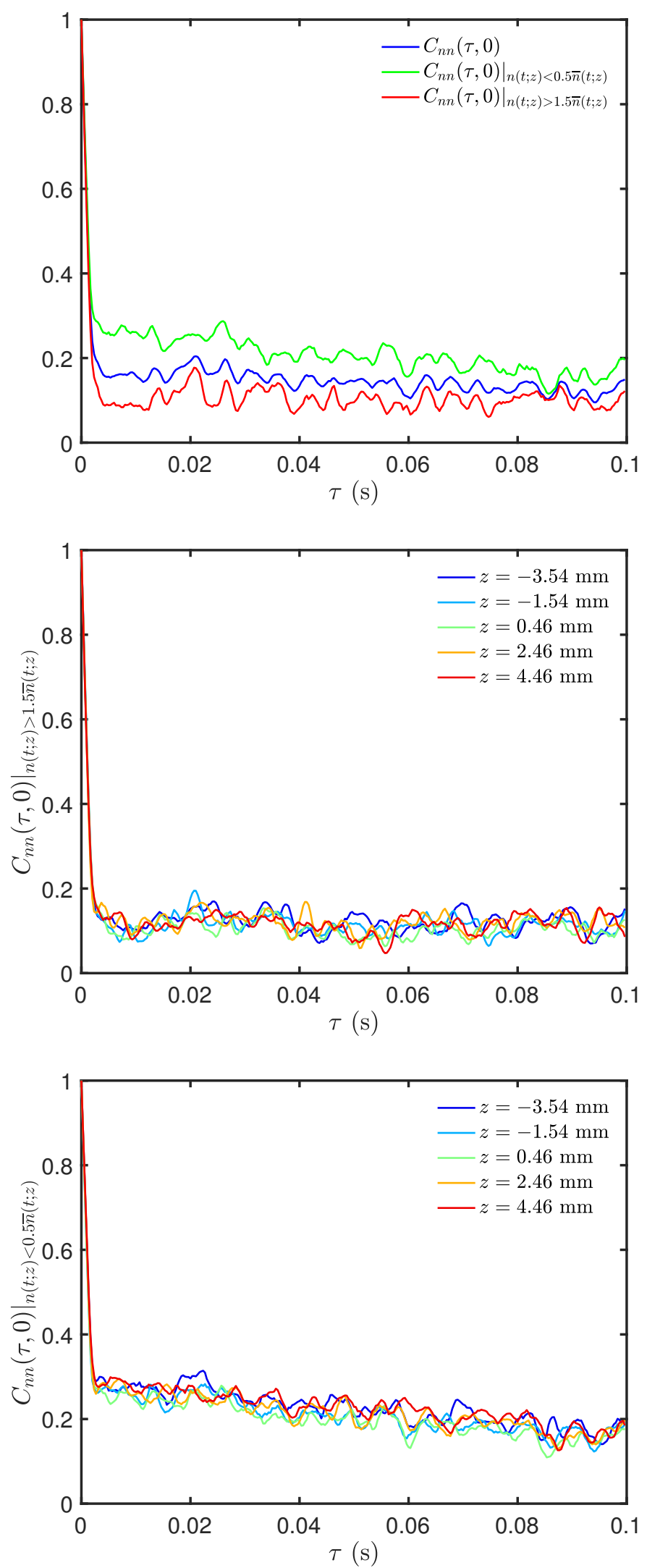

Figure 4.10.: $C_{n n}$ for DS2. Top: Limited to the central $\mathrm{mm}$ of the measurement volume, $C_{n n}$ is computed for the unconditional case in blue, for the dilute case in green, and the dense case in red. Middle (bottom) for mm-thick slices in the measurement volume, with the spectrum of colors refering to height $z$, for the dense (dilute) case. 

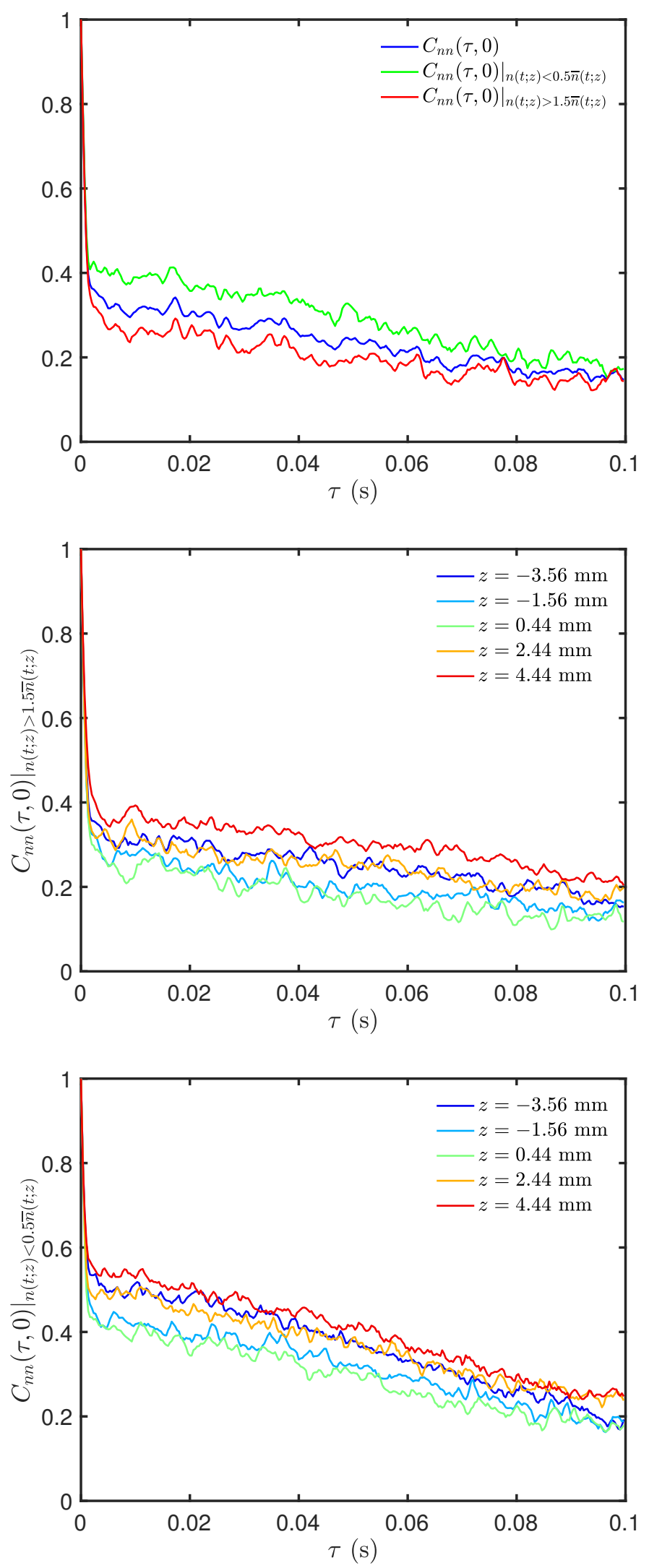

Figure 4.11.: Same as figure 4.10, but for DS3. 

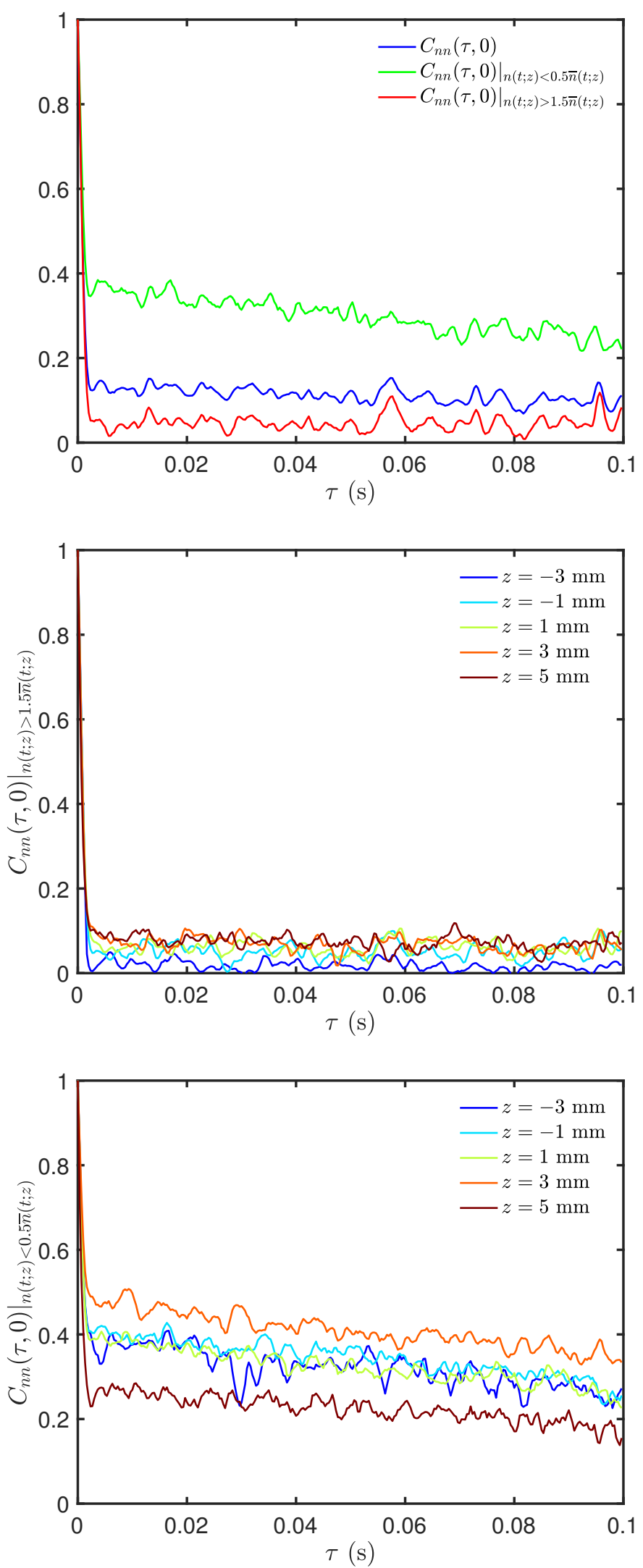

Figure 4.12.: Same as figure 4.10, but for DS4. 
The bottom two panels of these figures show the autocorrelation function of the dense (middle) and dilute (bottom) cases, for all cylindrical slices in the measurement volume. For each DS, the correlation time is shorter for the dense case by comparison to the dilute case. The relative brevity of the conditionally dense population's correlation time and its implications for particle clustering hold for the whole measurement volume, as shown in figures $4.13-4.15$. Here the autocorrelation function of the dilute (dense) case is divided by the unconditional case and is found, for all heights in the volume, to be greater than (less than) one. In other words, high local number-density always corresponds to a compact configuration, whereas low number density always occurs with a lower-thanaverage compactness. We also see that the ratio eventually returns to 1 at longer times. The interpretation of these figures is that the particles can reside in clumps. 

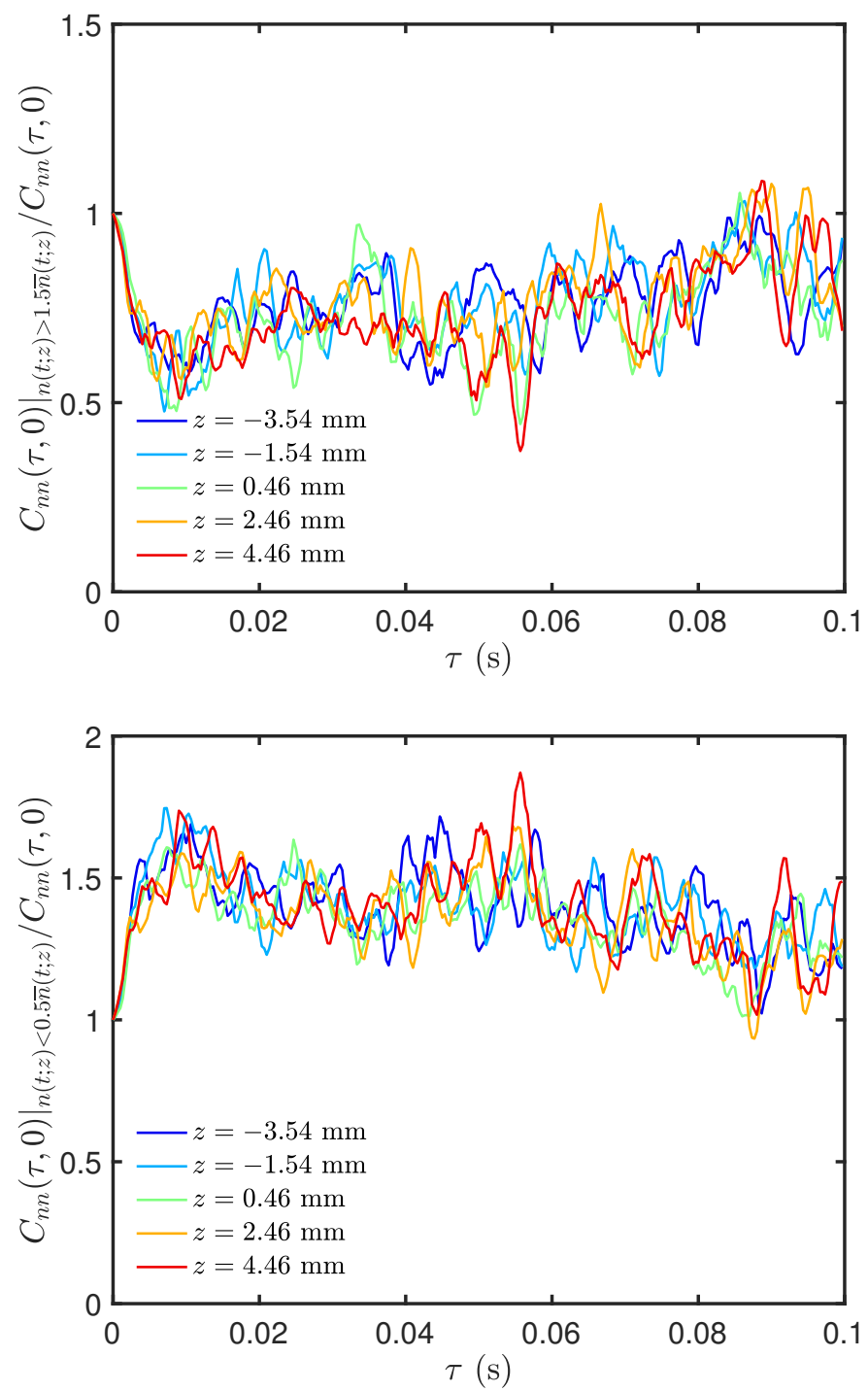

Figure 4.13.: $C_{n n}$ for the relatively dense (top) and relatively dilute (bottom) cases divided by the unconditional autocorrelation function. Colors as for figure 4.10. 

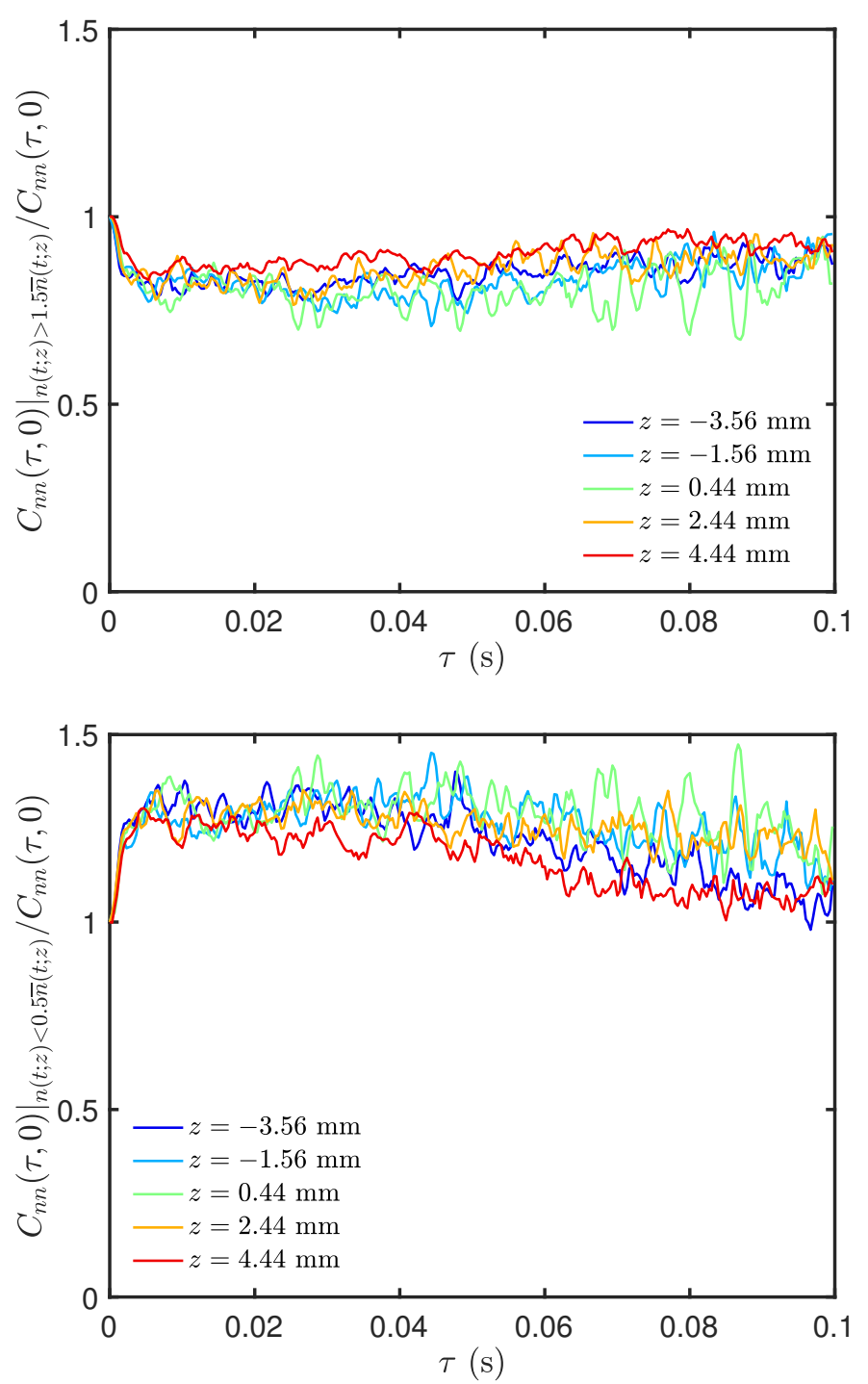

Figure 4.14.: Same as figure 4.13 but for DS3. 

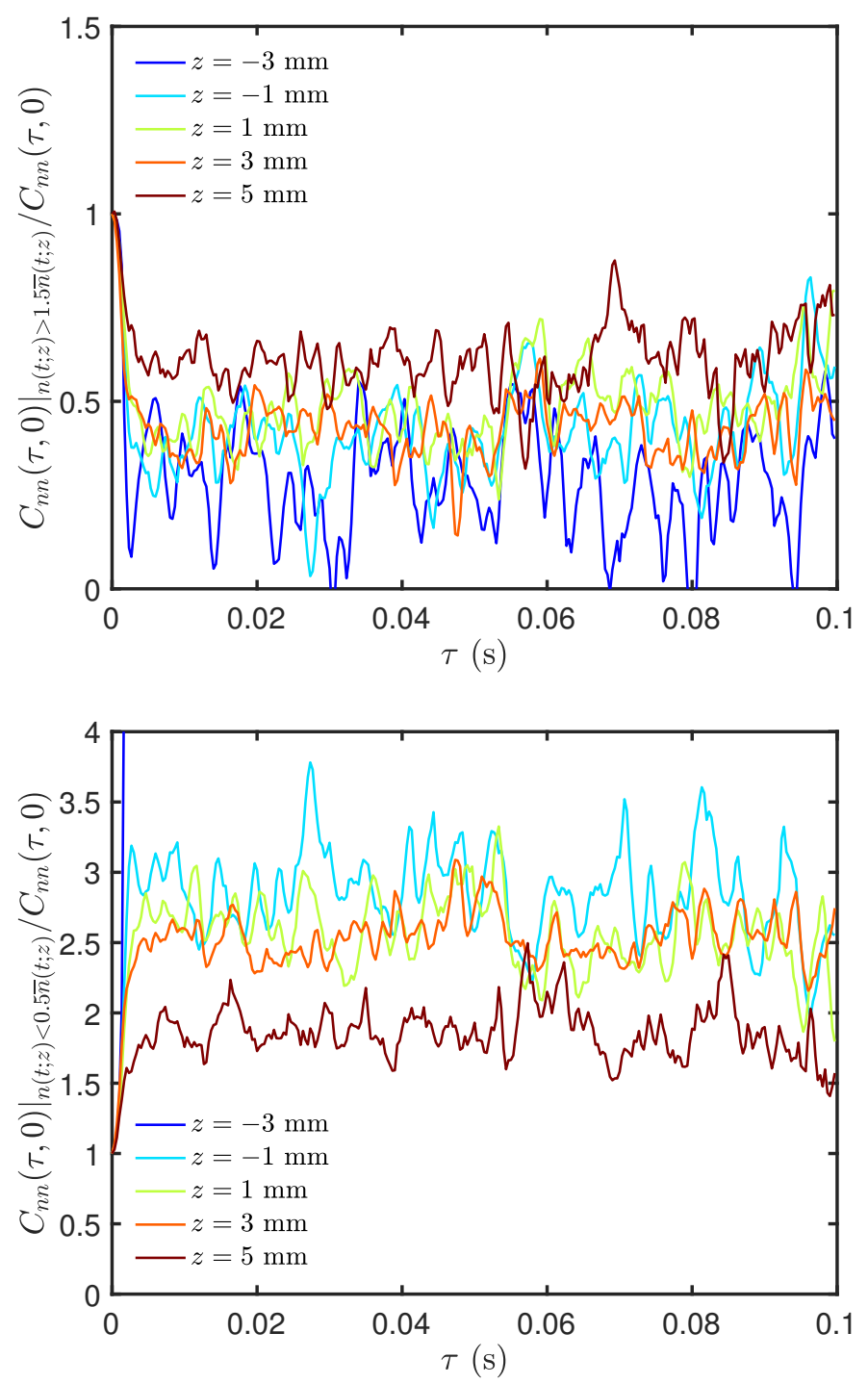

Figure 4.15.: Same as figure 4.13 but for DS4. 
Figure 4.16 shows that there tends to be a reduced mean vertical velocity for increased number of particles in a horizontal slice of one millimeter, in DS3 and DS4, which are the two DS that correspond to relatively high pressure. The same is not true for the lower pressure DS2. This seems to be a manifestation of collective particle drag reduction, since the direction of the drag force is coming from negative to positive $\mathrm{z}$ and the decreasing slope for DS3 and DS4 shows that particles resist being pushed upwards more readily when there are more of them.

The reader might also find it remarkable that there are sometimes up to 30 particles in a 1-mm slab, when the median total particle number in a volume about 10x this size (the whole measurement volume) is typically no more than $2 / 3$ this number. Comparing directly to the median particle number values shown in table 4.1, the presence of 30 particles in a 1-mm slab represents a local density enhancement above the background value $n$ by factors of 23 , 14 , and 60 , for DS2, DS3, and DS4, respectively. Yet, the values reported in the table are only median values, and the lower number density experiments, particularly in DS4, may not even have a total of 30 particles at any given time. Still, considering only the highest number-density experiment from this DS, with a mean of 10 particles and standard deviation of 4 , the range of local density enhancement factor is between 20-50. The large variability in the background value of $\mathrm{n}$ for DS3 is apparently because the mixture is extremely clumpy, in agreement with it being the highest mass-loading case. In appendix figures B.1- B.3, I show $n$ as a function of time for a few representative experiments in each dataset. The background level of $n$ is difficult to determine for the high mass-loading case, because the mean is not at all constant in time. 


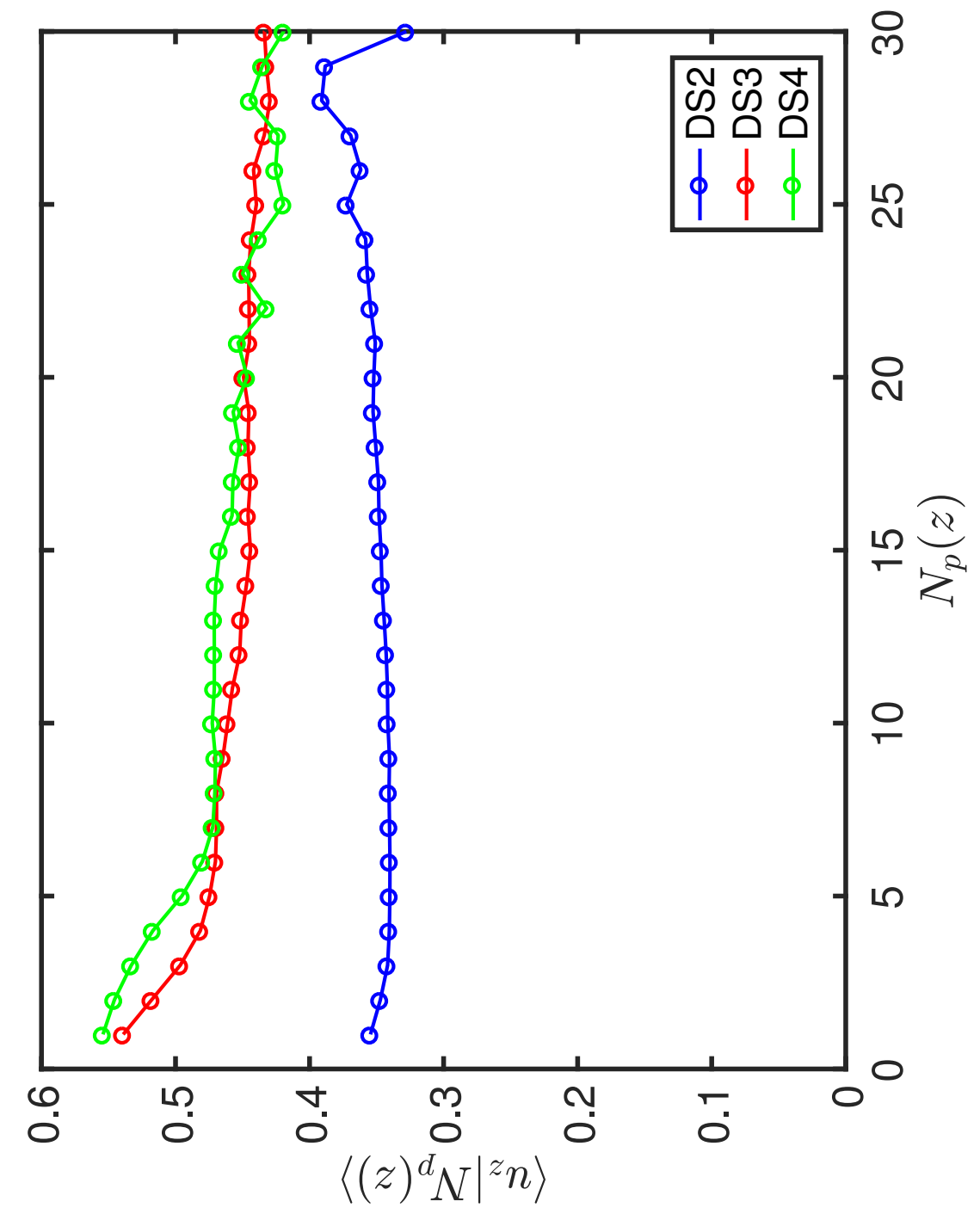

Figure 4.16: Mean settling velocity for DS2 in blue, DS3 in red, and DS4 in green, conditional upon the number of particles in a 1-mm cylindrical slab. 
The trend from 4.16 is mirrored in the probability distributions of the instantaneous vertical velocity within the small 1-mm slab, conditioned on the number of particles simultaneously present, shown in figure 4.17. Particularly in DS3 and DS4, the peak of the distribution function shifts towards lower velocities for larger number of particles. There is also a small shift of the peak in the PDF of DS2, however the distribution also becomes broader. 

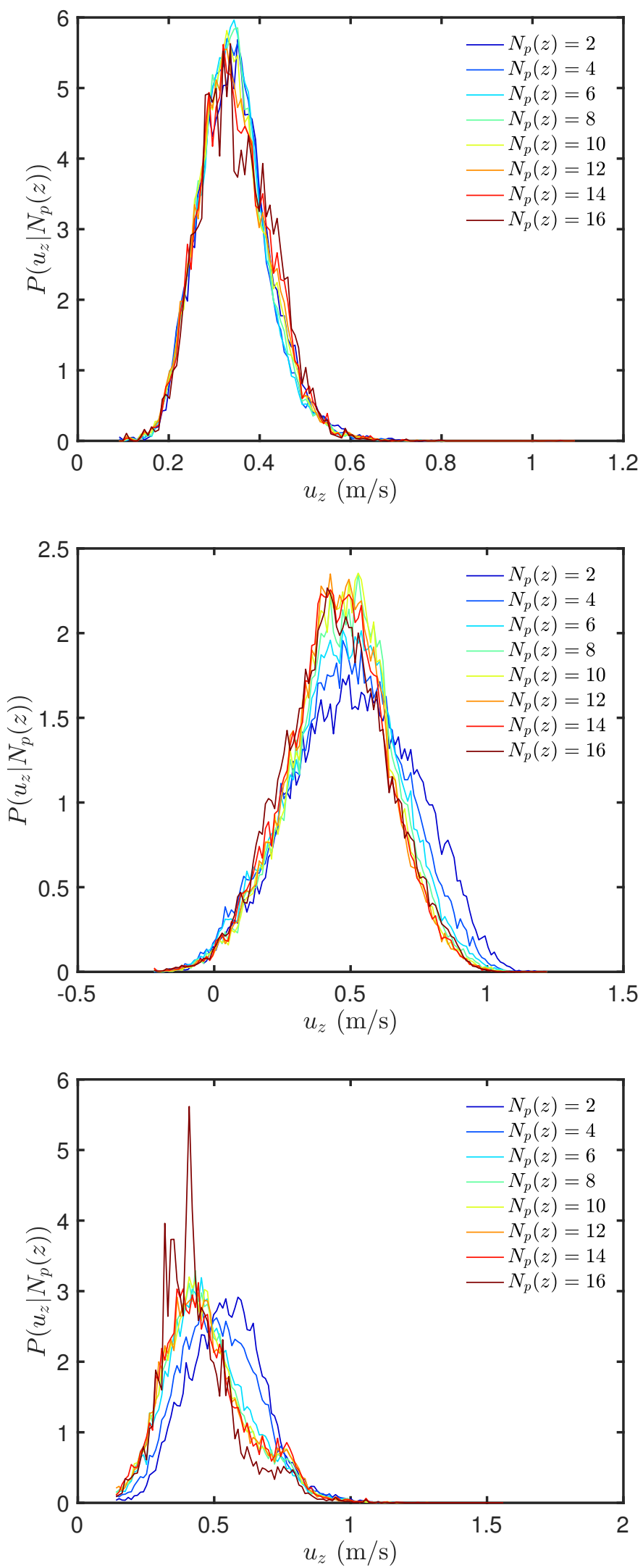

Figure 4.17.: PDFs of z-direction velocity, conditional upon the number of particles in a 1-mm slab, for DS2, DS3, and DS4, top, middle, bottom, respectively. 


\subsection{Global mass-loading dependencies}

The differences between the PDFs shown in figure 4.6 are emphasised by overlaying them on top of one another in figure 4.18 (for the velocity) and in figure 4.19 (for the acceleration). In addition to grouping the data according to gas pressure, I sort the experiments according to $n$, and divide into three subgroups of massloading (ML1, ML2, and ML3), which are indicated by vertical lines in figure 4.4. A feature of all velocity PDFs (again normalized by rms velocity or acceleration of the massloading bin), except the one corresponding to the highest pressure, is that the positive tail is accentuated. The extremely high values, around $u_{z}^{*}=7.5$, all belong to either ML1 or ML2, but not to the highest mass-loading bins. Note that DS 3 belongs to the highest massloading bins and so there is a trend towards damping of this tail with increasing massloading, at all pressures.

The acceleration pdfs also show various regimes that apparently depend upon pressure and number density, or equivalently on mass loading. For example, in DS2 (plus marks), the ML1 pdf lies on top of the DS1 PDF (i.e. isolated particles, all experiments in ML1), but the velocity PDF of DS2, ML2 is flatter and broader by comparison to DS2, ML2. The PDFS of the datasets at higher pressures (DS3 and DS4, squares and x's respectively) are in general broader. Some of the processes potentially responsible for these different regimes will be discussed in the final section of this thesis. 


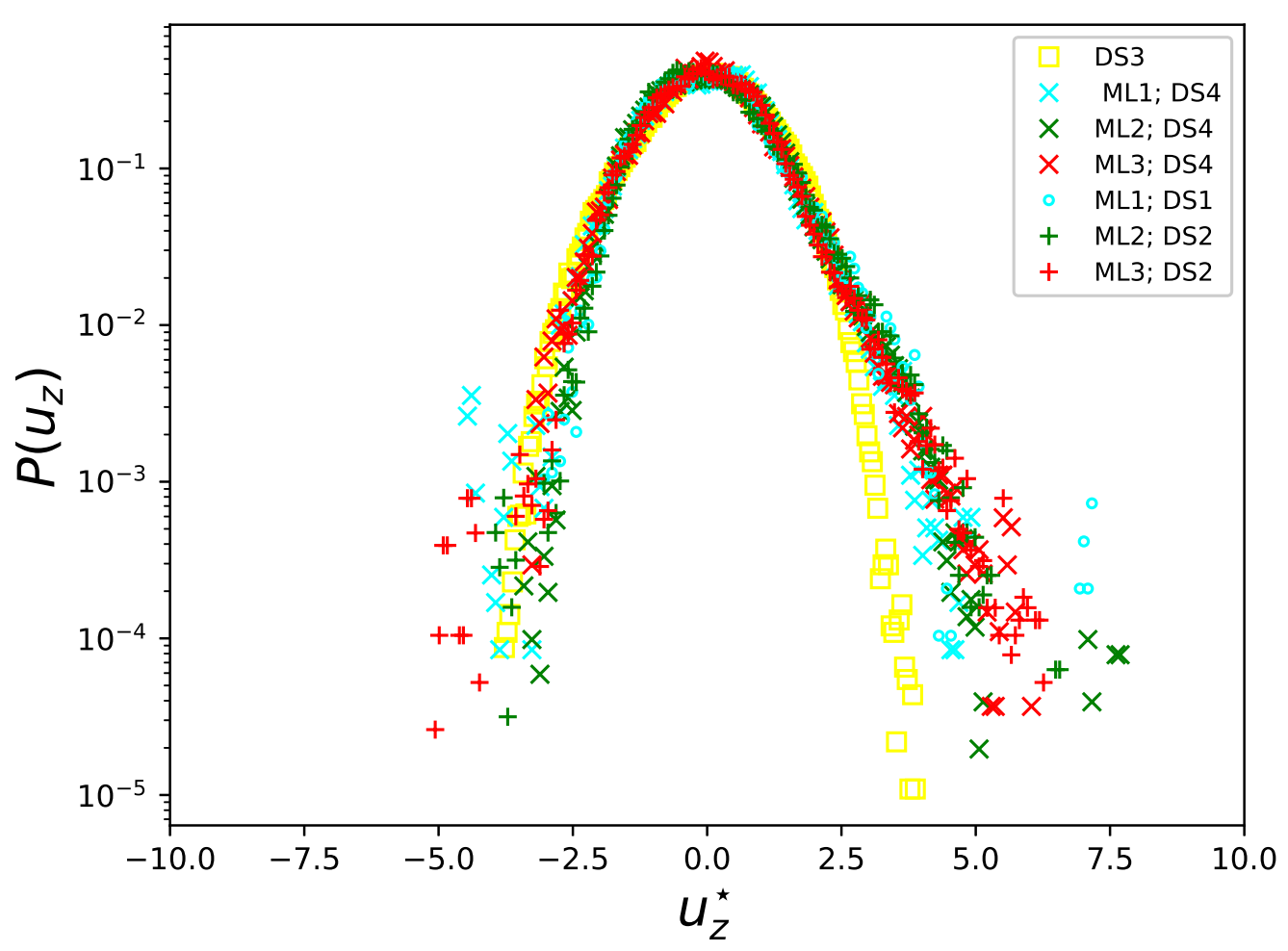

Figure 4.18.: Overlaid z-direction velocity PDFs sorted according to gas pressure and $\varepsilon$. DS1 is represented by circles, DS2 are represented by crosses, DS3 by squares and DS4 by x's. Mass-loading bin 1 is in cyan, mass-loading bin 2 is in green and mass-loading bin 3 is in Red. 


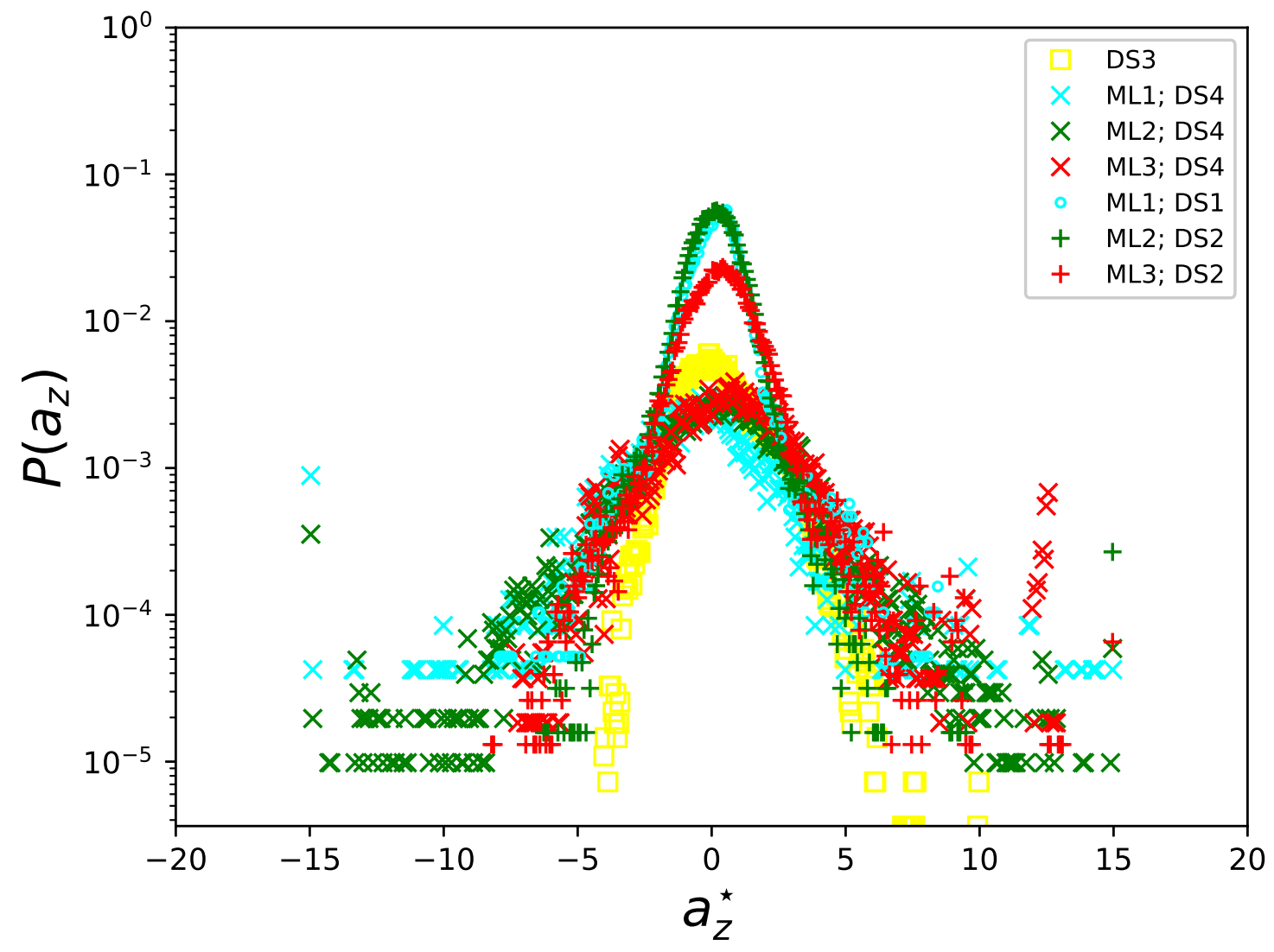

Figure 4.19.: Overlaid z-direction acceleration PDFs sorted according to gas pressure and $\varepsilon$. Colors and symbols same as for figure 4.18 


\section{CHAPTER 5}

\section{Theoretical Results}

\subsection{Simulations}

To circumvent the complexities added by rotation, in Lambrechts et al. (2016), we considered a sedimenting particle suspension in the absence of rotational shear. The two-fluid model was the same as that described previously, except the body forces in equation 1.16 included $g$ and there was a hydrostatic pressure gradient instead of a globally driven one. To justify using the two-fluid model, we required that the momentum diffusion time scale is longer than the particle friction time ${ }^{1}$. The units of the system were described in terms of $T_{f}$, with velocity units given by equation 1.11, and length units $l_{f}=g T_{f}^{2}$ so that the results could be scaled arbitrarily.

We found a similar effect as was noticed in previous studies of the SI: namely, particle clumping and apparently reduced collective particle drag for over-dense regions.

The choice of dimensionless units was motivated by our wish to compare the results to the

\footnotetext{
${ }^{1}$ Whereas in other studies it was justified by assuming that drifting particles adjust hydrostatically against the pressure gradient.
} 
ground-based laboratory flow investigated in the present thesis. In that work, we scaled the results to a local patch of a PPD, in the limit of large $R o$, and assumed that the context was the vertical settling of dust grains towards the disc mid plane. We also briefly considered how the effect might alter the dust opacity and therefore the thermal balance in the atmosphere of an accreting giant planet. A potentially important outcome from the simplified model is that the effect may be more general than previously thought, and therefore could apply to a host of circumstances. In studying a system in terms of its dimensionless control parameters, one evokes the principle of similarity, commonly used to scale fluid-dynamics models to diverse applications; experimental flows in the same flow regime as a PPD should exhibit similar velocity fields, even if the exact conditions are rather different. 
A version of the following was published in Astronomy and Astrophysics (A\&A), Volume 591, Article number A133, Year 2016, as 'Spontaneous concentrations of solids through two-way drag forces between gas and sedimenting particles', by M. Lambrechts, A. Johansen, H. L. Capelo, J. Blum, and E. Bodenshatz.

\subsection{Abstract}

The behaviour of sedimenting particles depends on the dust-to-gas ratio of the fluid. Linear stability analysis shows that solids settling in the Epstein drag regime would remain homogeneously distributed in non-rotating incompressible fluids, even when dust-to-gas ratios reach unity. However, the non-linear evolution has not been probed before. Here, we present numerical calculations indicating that in a particle-dense mixture solids spontaneously mix out of the fluid and form swarms overdense in particles by at least a factor 10 . The instability is caused by mass-loaded regions locally breaking the equilibrium background stratification. The driving mechanism depends on non-linear perturbations of the background flow and shares some similarity to the streaming instability in accretion discs. The resulting particlerich swarms may stimulate particle growth by coagulation. In the context of protoplanetary discs, the instability could be relevant for aiding small particles to settle to the midplane in the outer disc. Inside the gas envelopes of protoplanets, enhanced settling may lead to a reduced dust opacity, which facilitates the contraction of the envelope. We show that the relevant physical set up can be recreated in a laboratory setting. This will allow our numerical calculations to be investigated experimentally in the future. 


\subsection{Introduction}

The study of gas drag on mm to dm-sized particles (pebbles) is essential to understand the formation of planets. Vertical sedimentation due to drag on small particles in the protoplanetary disc is necessary for the creation of a dense midplane of solids from which larger objects can grow (Youdin \& Lithwick 2007). Conversely, the same drag force is also responsible for the rapid radial migration of pebbles in the midplane (on $100 \mathrm{yr}$ time scales in the terrestrial region, Weidenschilling 1977a). This is the main barrier for continued growth to larger than $\mathrm{cm}$ to $\mathrm{m}$ sizes by collisions (Brauer et al. 2008b, Birnstiel et al. 2012), unless particles can have extremely low internal densities (Kataoka et al. 2013, Krijt et al. 2015).

The radial drift hurdle can be avoided through two mechanisms that also critically rely on gas drag. Firstly, pebbles can be concentrated hydrodynamically, so that the resulting clouds collapse gravitationally to planetesimals of $\sim 100 \mathrm{~km}$ in size (for recent reviews on different planetesimal formation models, see Johansen et al. 2014, Chiang \& Youdin 2010). Secondly, large planetesimals can accrete the remaining drifting pebbles and grow to planetary sizes (Lambrechts \& Johansen 2012, 2014, Guillot et al. 2014).

Not only the drag on the particles is important, but also the backreaction of the particles on the gas. Initially, it was proposed that a secular instability on a settled dust layer could lead to local particle pileups (Goodman \& Pindor 2000). The pileup would originate from a process resembling plate drag, where the drag force is assumed to collectively act on a monolithic particle midplane. This assumption is nevertheless questionable (Youdin \& Chiang 2004) and numerical studies (Weidenschilling 2006) have not recovered the instability proposed by Goodman \& Pindor (2000). Nevertheless, this work paved the way for a further investigation on the role of the backreaction force from gas drag. Youdin \& Goodman (2005) identified a linear instability in the disc midplane. Their breakthrough result demonstrated that infinitesimal perturbations grow on an orbital time-scale when the dust-to-gas ratio is 
around unity or higher. This instability leads to spontaneous particle clumping, triggering the gravitational collapse that results in the formation of planetesimals. In a series of papers (Youdin \& Johansen 2007, Johansen \& Youdin 2007, Johansen et al. 2009, 2012) the linear and non-linear evolution of this instability were numerically investigated in detail. These results were independently confirmed and further explored by several other groups (Bai \& Stone 2010b,a,c, Miniati 2010, Kowalik et al. 2013).

Several criteria for the streaming instability to achieve particle clumping have been identified:

- a disc with slightly supersolar dust-to-gas ratio (Johansen et al. 2009, Bai \& Stone 2010c),

- particles of Stokes number $\tau_{\mathrm{f}} \sim 0.05-0.5$, approximately between $\mathrm{mm}$ and $\mathrm{dm}$ in size (Johansen \& Youdin 2007, Bai \& Stone 2010b,c, Carrera et al. 2015) and

- low radial pressure support in the disc (Bai \& Stone 2010c).

Further investigations are moving towards a more global understanding of the effects of the streaming instability, by expanding the simulation domain in the azimuthal (Kowalik et al. 2013) or vertical direction (Yang \& Johansen 2014). Additionally the streaming instability is placed in a larger context by incorporating magnetized turbulence (Johansen et al. 2007), dust coagulation models (Dra̧żkowska \& Dullemond 2014) or vortex formation (Raettig et al. 2015).

In this paper we take a step back and study the general process of particle sedimentation in flows with a dust loading comparable to the gas density. The aim is twofold. Firstly, we hope to gain theoretical insight into particle sedimentation and more complex drag instabilities, such as the streaming instability and the photoelectric instability (Lyra \& Kuchner 2013). Secondly, the sedimentation of particles is accessible to laboratory experiments, thus allowing for a potential experimental confirmation of a particle drag instability. 
Of specific interest is the question whether any particle clumping will even occur at all in a mass-loaded particle rain. From previous analytic work on the streaming instability, we do not expect a linear instability to be present, because of the lack of rotation in a pure sedimentation problem. This removes the Coriolis force which is deemed necessary for the streaming instability to operate (Jacquet et al. 2011, Youdin \& Goodman 2005). Nevertheless, in our physical set up (described in Section 5.4) a non-linear drafting instability is clearly present. The results are described in Section 5.5. The implications of this instability are discussed for particle sedimentation in protoplanetary discs, chondrule formation, and the envelopes of giant planets (Section 5.6). We also place our results in the context of planned laboratory experiments (Section 2.2). We summarise our findings in Section 5.8.

\subsection{Mass-loaded particle rain}

\subsubsection{Model equations}

We study the differential motion between particles initially moving with terminal velocity and stationary gas in hydrostatic balance. The dynamics of the gas component is described by

$$
\begin{aligned}
\partial_{t} \rho+\nabla \cdot(\rho u) & =0, \\
\partial_{t} u+u \nabla \cdot u & =-g e_{z}-\frac{1}{\rho} \nabla P+\frac{1}{t_{\mathrm{f}}} \varepsilon(v-u)+v \nabla^{2} u,
\end{aligned}
$$

where $\rho$ is the gas density, $u$ the gas velocity, $g$ the gravitational acceleration, $P$ the pressure, $\varepsilon=\rho_{\mathrm{p}} / \rho$ is the local dust-to-gas ratio, $v$ the particle fluid velocity and $v$ the viscosity. The drag term from the particles onto the gas depends on the friction time of the particle (in the 
Epstein drag regime, Epstein 1924),

$$
t_{\mathrm{f}}=\frac{\rho_{\bullet} R}{\rho v_{\mathrm{th}}},
$$

where $R, \rho_{\bullet}$ are the radius and solid density of the particle. The thermal velocity $v_{\text {th }}$ is approximately equal to the local, isothermal gas sound speed $v_{\text {th }}=\sqrt{8 / \pi} c_{\mathrm{s}}$.

We investigate a regime where we assume an efficient coupling between particles and gas. In this case, the viscous diffusion time for momentum transport between an average particle pair located a distance $l_{\text {pair }}$ apart is shorter than the friction time of a single particle,

$$
t_{v, \text { pair }}=\frac{l_{\text {pair }}^{2}}{v}=\frac{n_{\mathrm{p}}^{-2 / 3}}{v}<t_{\mathrm{f}}
$$

with $n_{\mathrm{p}}$ the particle number density. The particles can then be described by a pressureless fluid (a formal derivation can be found in Jacquet et al. 2011) with continuity and momentum equation

$$
\begin{aligned}
\partial_{t} \rho_{\mathrm{p}}+\nabla \cdot\left(\rho_{\mathrm{p}} v\right) & =0, \\
\partial_{t} v+v \nabla \cdot v & =-g e_{z}-\frac{1}{t_{\mathrm{f}}}(v-u) .
\end{aligned}
$$

In the remainder of the paper we will employ 'friction units': the friction time $t_{\mathrm{f}}$ as time unit and the friction length $l_{\mathrm{f}}=g t_{\mathrm{f}}^{2}$ as length unit. Velocities can then be expressed in units of terminal velocity $v_{\mathrm{f}}=g t_{\mathrm{f}}$. The criterion expressed in Eq. (5.4), for example, reduces to $n_{\mathrm{p}}^{\prime-2 / 3} / v^{\prime}<1$. We will preserve the prime notation in the following sections to explicitly denote quantities expressed in friction units.

The use of the friction time as unit of time is possible for the sedimentation problem, because there are no rotation terms that would necessarily introduce the additional time scale of the orbital Keplerian frequency, as is for example the case for the streaming instability 
(Youdin \& Goodman 2005).

Expressed in friction units the model equations leave us with only three free dimensionless parameters:

- the viscosity, $v^{\prime}=v /\left(g^{2} t_{\mathrm{f}}^{3}\right)$, which is the inverse of the Reynolds number $(R e)$ in terms of the terminal velocity and the friction length,

- the sound speed, $c_{\mathrm{s}}^{\prime}=c_{\mathrm{s}} /\left(g t_{\mathrm{f}}\right)$, which is the inverse of the Mach number $(M a)$ in terms of the terminal velocity, and

- the dust-to-gas ratio, $\varepsilon$.

The latter is arguably the most important, because we desire to work in the incompressible limit $(M a \ll 1)$ and we face a lower bound on the viscosity imposed by the requirement for numerical stability.

A major benefit from this choice of units is that our calculations do not require us to specify a particle size (Eq. 5.3). Thus our results can be freely scaled to the desired particle size in the context of protoplanetary discs (Section 5.6) or a laboratory setting (Section 2.2).

\subsubsection{Numerical implementation}

In our numerical simulations, performed with the Pencil Code ${ }^{2}$, we do not employ the particle fluid description used for the analytical calculations for our main results, but instead use a Lagrangian super-particle approach. Particles are implemented as super-particles that represent swarms of physical particles. This is important, especially in the non-linear regime where it is desirable to allow particle trajectory crossing and steep density gradients (Youdin $\&$ Johansen 2007). We have nevertheless used the particle fluid approach also numerically to

\footnotetext{
${ }^{2}$ The Pencil Code is open source and can be obtained at

http://pencil-code.nordita.org/. A description of the code can be found in Brandenburg \& Dobler (2002b), Brandenburg (2003) and Youdin \& Johansen (2007).
} 
verify some of our work in the linear regime. The assignment of drag forces on the particles and on the gas is further described in Youdin \& Johansen (2007) and in Johansen \& Youdin (2007). Drag is calculated on particles assuming a constant friction time. We have also made use of the block domain decomposition for particle load balancing amongst processors (Johansen et al. 2011).

For the initial condition, we set up a gas column in hydrostatic equilibrium, taking the drag from the particles on the gas into account. Particles are typically distributed randomly, with the possibility to add perturbations (discussed in more detail in Section 5.5.1) and initiated with with their vertical velocity equal to the terminal velocity. The gas stratification then takes the form

$$
\rho=\left(\rho_{p}+\rho_{\mathrm{b}}\right) \exp \left(-\frac{g}{c_{s}^{2}} z\right)-\rho_{p},
$$

see Appendix 5.9 for more details. This initial condition works well, but we nevertheless find that in heavily elongated simulation domains, the stratification of the gas combined with a uniform particle distribution triggers a vertical gas density wave, that dissipates over time. There is also a small upwards advection of gas as the top of the domain gets cleared of particles. These effects restricts our simulation domain in practice to approximately $20 l_{\mathrm{f}}$ in the vertical direction (at $c_{\mathrm{s}} / v_{\mathrm{f}}=10$ ).

A full list of the performed simulations can be found in Table 5.1 and Table 5.2. Below, we describe the nominal numerical set up in detail.

The boundary condition are set to be periodic in the horizontal direction, for both the particle and gas component. In the vertical direction particles are removed from the simulation when crossing the edge of the simulated domain. The vertical boundary condition on the gas is symmetric (vanishing first derivative) in all quantities, except for the vertical gas velocity which is antisymmetric (vanishing value). This boundary condition effectively puts a solid 
surface at the bottom of the simulation domain, on which the gas is supported.

We use an ideal gas equation of state with adiabatic index $\gamma=5 / 3$. The sound speed is set to be $c_{\mathrm{s}}=10 v_{\mathrm{f}}$ (unless mentioned otherwise) to approach the incompressible limit. The Pencil Code is a code optimised for both subsonic and mildly transsonic flows, but we found a Mach number of 0.1 sufficient to probe the incompressible regime of interest.

We found a choice of 16 superparticles per grid cell is sufficiently high to model a coherent fluid and reduce particle noise (see also Appendix 5.11). We have standardly used a physical viscosity treatment, but for runs with extended domain and high mass loading (run1.01, run1.02) we added sixth order 'artificial viscosity' (Haugen \& Brandenburg 2004). We employed the minimal amount necessary to prevent numerical artefacts from developing. The grid Reynolds number is minimally 32 times smaller than the Reynolds number in friction units. Most simulations were performed in 2 dimensions, but we have verified our results in 3 dimensions as well (run3d.4, Fig. 5.2), showing little difference. Nevertheless, such numerically expensive 3D runs are of interest for further study. 
Table 5.1.: Parameters of the numerical simulations. All values are given in the friction unit system. Here, $v$ is the viscosity, $\varepsilon_{0}$ is the background dust-to-gas ratio, $c_{\mathrm{s}}$ is the sound speed, $A$ and $\lambda$ are the values of the amplitude and wavelength of the perturbation. The total number of particles is $N_{\text {par }}$ and $N_{\text {cells }}$ is the total number of grid cells.

\begin{tabular}{cccccccccc}
\hline \hline Name & $L_{x} \times L_{z}$ & Resolution & $v$ & $\varepsilon_{0}$ & $c_{\mathrm{S}}$ & Perturb. & $A$ & $\lambda$ & $N_{\text {par }} / N_{\text {cells }}$ \\
\hline run1 & $1 \times 20$ & $32 \times 640$ & $1.0 \mathrm{e}-4$ & 1 & 10 & rand & - & - & 16 \\
run2 & $1 \times 20$ & $64 \times 1280$ & $1.0 \mathrm{e}-4$ & 1 & 10 & rand & - & - & 16 \\
run3 & $1 \times 20$ & $128 \times 2560$ & $1.0 \mathrm{e}-4$ & 1 & 10 & rand & - & - & 16 \\
runRT & $1 \times 20$ & $32 \times 640$ & $1.0 \mathrm{e}-4$ & 1 & 10 & $k_{\mathrm{z}}$ & 0.1 & 4 & 16 \\
runKH & $2 \times 20$ & $64 \times 640$ & $1.0 \mathrm{e}-4$ & 1 & 10 & $k_{\mathrm{x}}$ & 0.1 & 0.5 & 16 \\
runEGG & $1 \times 20$ & $32 \times 640$ & $1.0 \mathrm{e}-4$ & 1 & 10 & eggbox & 0.1 & 1,4 & 16 \\
runEGG2 & $1 \times 20$ & $64 \times 1280$ & $1.0 \mathrm{e}-4$ & 1 & 10 & eggbox & 0.1 & $0.5,2$ & 16 \\
runv2 & $1 \times 20$ & $32 \times 640$ & $1.0 \mathrm{e}-2$ & 1 & 10 & rand & - & - & 16 \\
runv3 & $1 \times 20$ & $32 \times 640$ & $1.0 \mathrm{e}-3$ & 1 & 10 & rand & - & - & 16 \\
runv5 & $1 \times 20$ & $32 \times 640$ & $1.0 \mathrm{e}-5$ & 1 & 10 & rand & - & - & 16 \\
runv6 & $1 \times 20$ & $32 \times 640$ & $1.0 \mathrm{e}-5$ & 1 & 10 & rand & - & - & 16 \\
run1.e4 & $1 \times 20$ & $32 \times 640$ & $1.0 \mathrm{e}-4$ & 1 & 10 & rand & - & - & 16 \\
run2.n4 & $1 \times 20$ & $64 \times 1280$ & $1.0 \mathrm{e}-4$ & 1 & 10 & rand & - & - & 4 \\
run2.n64 & $1 \times 20$ & $64 \times 1280$ & $1.0 \mathrm{e}-4$ & 1 & 10 & rand & - & - & 64 \\
run3d.4 & $1 \times 1 \times 20$ & $32 \times 32 \times 640$ & $1.0 \mathrm{e}-4$ & 4 & 10 & rand & - & - & 16 \\
\hline
\end{tabular}

Table 5.2.: Parameters of the numerical simulations extended in the vertical domain by $t_{\mathrm{f}}=0.1$. Values of variables in friction units (similar to Table 5.1). Here, art. visc. stands for the value of the artificial viscosity parameter.

\begin{tabular}{ccccccccccc}
\hline \hline Name & $L_{x} \times L_{z}$ & Resolution & $v$ & $\varepsilon_{0}$ & $c_{\mathrm{s}}$ & Perturb. & $\mathrm{A}$ & $\lambda$ & $N_{\text {par }} / N_{\text {cells }}$ & art. visc. \\
\hline run1.01 & $100 \times 2000$ & $32 \times 640$ & $1.0 \mathrm{e}-1$ & 1 & 100 & rand & - & - & 16 & 10 \\
$\operatorname{run} 2.01$ & $100 \times 2000$ & $32 \times 640$ & $1.0 \mathrm{e}-1$ & 0.25 & 100 & rand & - & - & 16 & 10 \\
$\operatorname{run} 3.01$ & $100 \times 2000$ & $32 \times 640$ & $1.0 \mathrm{e}-1$ & 0.1 & 100 & rand & - & - & 16 & - \\
$\operatorname{run} 4.01$ & $100 \times 2000$ & $32 \times 640$ & $1.0 \mathrm{e}-1$ & 0.05 & 100 & rand & - & - & 16 & - \\
\hline
\end{tabular}




\subsection{Numerical results showing spontaneous particle concentrations}

\subsubsection{Nonlinear behaviour}

From previous analytic studies of the streaming instability (Youdin \& Goodman 2005, Jacquet et al. 2011), it is well known that rotation is an essential ingredient for the linear phase of particle clumping. The interpretation proposed by Jacquet et al. (2011) is that the Coriolis force is necessary to create a pressure maximum supported by geostrophic balance. We have repeated the analysis in Appendix 5.9 for completeness. It demonstrates that the sedimentation model without rotation discussed in this paper is not expected to show a linear instability, under the assumption of incompressible gas.

Our numerical results nevertheless show that even a minimal disturbance of the sedimenting particle component with $\varepsilon=1$ leads to spontaneous clumping of material, resulting in the particles to unmix and sediment out of the fluid. Figure 5.1 illustrates the process. Particles located in initially weakly overdense regions sediment faster, dragging the gas along, resulting in a drafting effect which pulls in more particles. For clarity, the drafting mentioned here is the result of the collective motion of a swarm of particles and the resulting gas drag back reaction, not from individual particle slipstreaming. At the same time, the opposite occurs in regions less dense than the mean particle density. These effects amplify each other, which results in dense swarms of particles to form that can undergo secondary instabilities. For example, one can see in the bottom of the last panel of Fig. 5.1 a particle cloud resembling a characteristic Rayleigh-Taylor mushroom, which we will discuss in more detail below. 


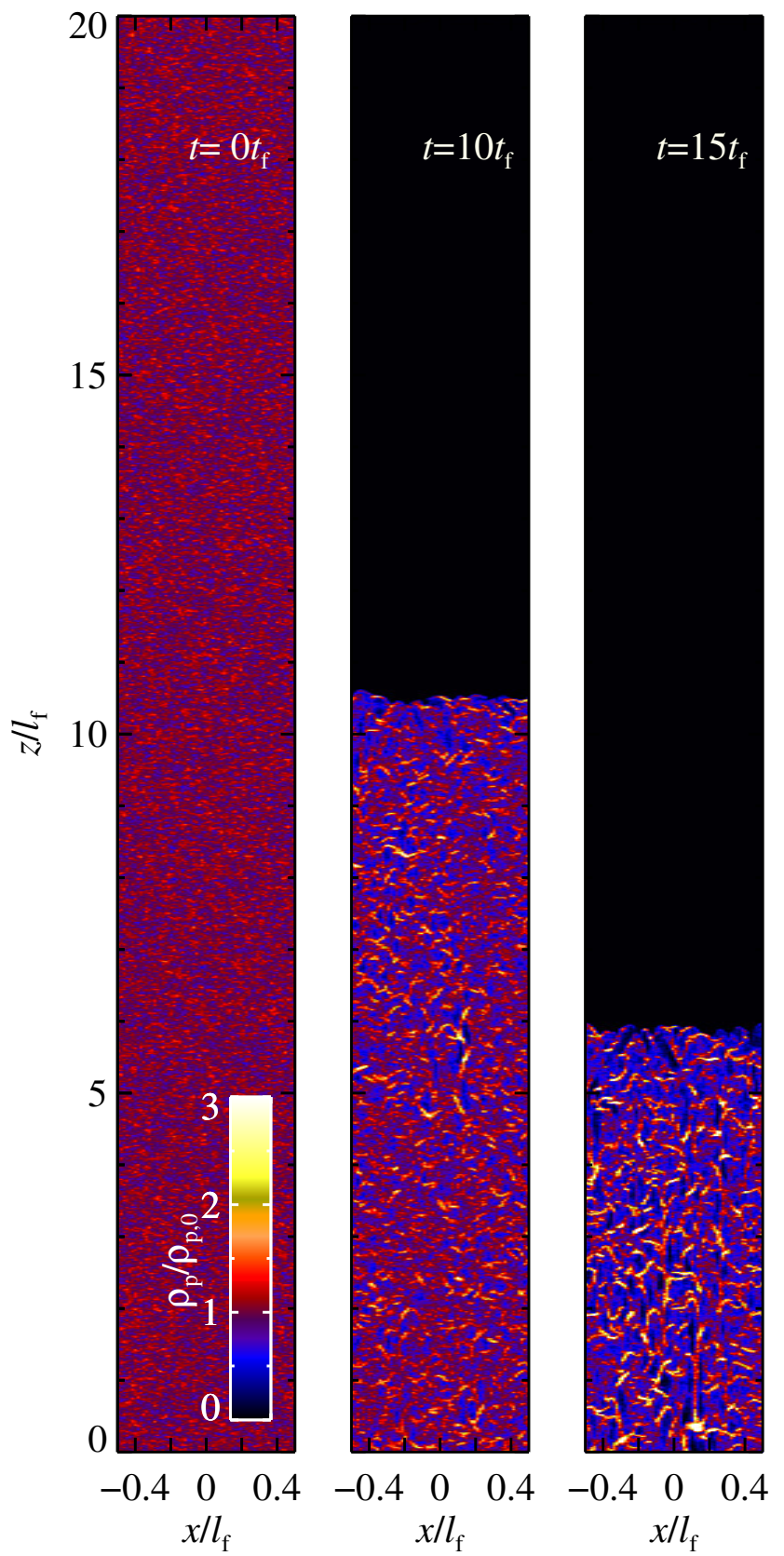

Figure 5.1.: Development of particle swarms by a drafting instability. Displayed is the evolution of the particle density in the two-dimensional simulation run2 (see Table 5.1). The left-most panel illustrates the initial conditions: a stratified gas column in the vertical directions with particles sedimenting at terminal velocity, placed randomly throughout the simulation domain $\left(1 l_{\mathrm{f}}\right.$ wide and $20 l_{\mathrm{f}}$ high, note that the figure aspect ratio is enlarged in the $x$-direction). In the following panels (time $t=10,15 t_{\mathrm{f}}$ ), regions marginally overdense with particles locally break the stratification equilibrium and accelerate downwards, while in particle-poor regions a deceleration from terminal velocity occurs. This leads to continued particle pileups through drafting, originating from gas being dragged by the particles. The particle and fluid components unmix and remain in this state. At the end of the simulation, when most particles have sedimented out of the simulation domain, the maximal particle density has increased by a factor 10 . 


\subsubsection{Particle noise perturbation}

We begin by considering the evolution of the sedimenting particles, when they are placed randomly in the simulation domain. This corresponds to a noisy initial condition for the particle distribution, with maximal relative changes in the particle density on the order of $50 \%$ for our nominal 2D resolution, for more detail see Appendix 5.11.

Figure 5.2 shows the time evolution of the maximal particle density in the simulated domain (for different background dust-to-gas ratios). Particle overdensities reach 10 times the average value, although they are still growing slowly towards the end of the simulations when the particles fall out of the box. Over time, particles sediment out of the simulated domain, so at late times fewer and fewer particles are traced. For clarity, we also show the evolution of the horizontal gas velocity dispersion, which is a less noisy measurement than the particle density. This illustrates the exponential nature of the instability as well.

Our numerical results stand in contrast to the stable state predicted by linear stability analysis. It appears that the main driver for the particle clumping is an imbalanced stratification. Recall that the particle loading of the sedimenting particles is taken into account when setting up the equilibrium state (Eq.5.7). This balance can be broken along the $x$-direction by regions with a higher, or less high, particle density compared to the mean value. Apparently, the fluid and particles have no means of finding a global equilibrium state in the $x$-direction in the response to the particle fluctuation. Instead, the particle components breaks into dense swarms.

This interpretation is supported by the correlation between overdense regions and their increased settling speeds shown in Fig. 5.3. We have binned the surrounding gas and particle velocity in the grid cell for every particle in the simulation of run2, revealing that on average gas and particles sediment about $1 \%$ of faster or slower for order unity fluctuations in the dust-to-gas ratio. 

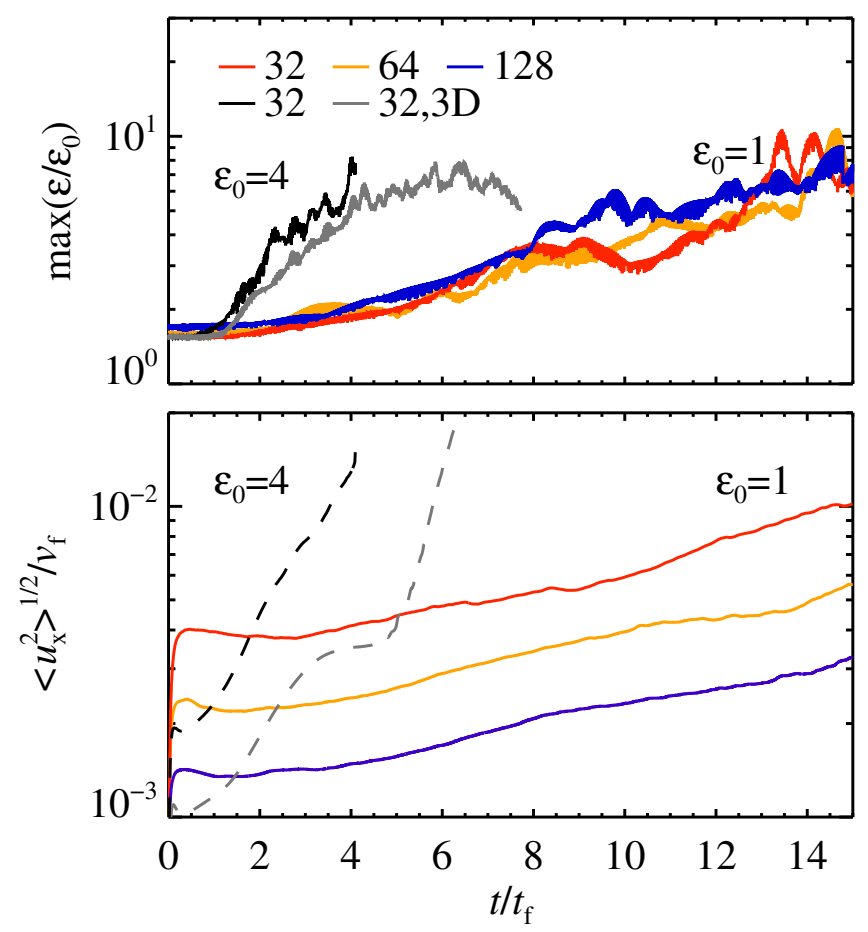

Figure 5.2.: Top panel: Evolution of the particle density (normalized to the background dustto-gas ratio $\varepsilon_{0}$ ), for three different resolutions in 2D simulations and a $3 \mathrm{D}$ simulation (run $3 \mathrm{~d} .4$, gray). Bottom panel: Evolution of the instability based on the horizontal gas dispersion, $\sqrt{\left\langle u_{x}^{2}\right\rangle}$, (as an alternative tracer to the maximal particle density, which is an intrinsically noisy variable). We have used a similar color coding as top panel. Note for the runs with $\varepsilon_{0}=4$ we have displaced the dashed curves for clarity with a factor 10 downwards. 


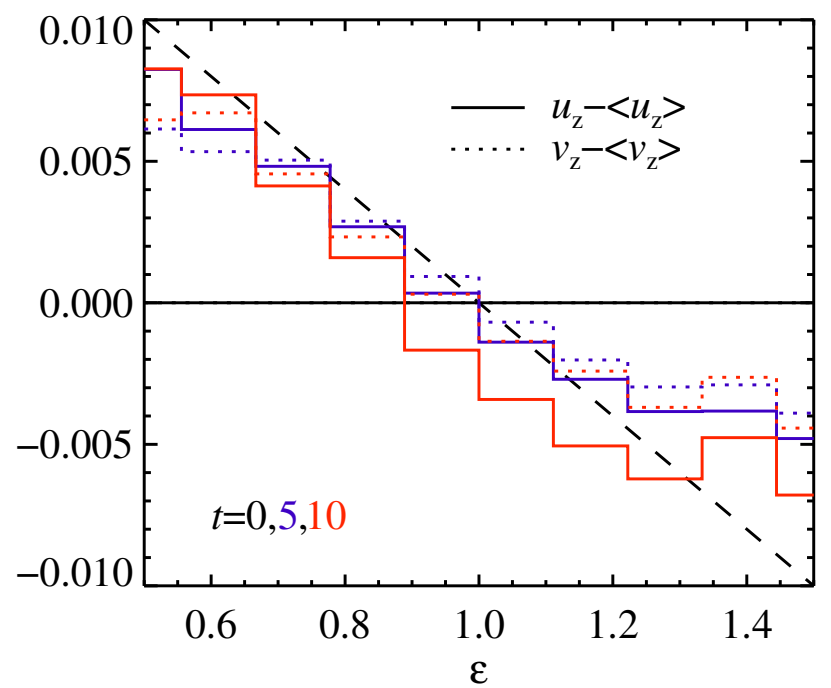

Figure 5.3.: Correlation between the local dust-to-gas ratio $\varepsilon$ and the deviations in the velocity of the gas $u_{\mathrm{z}}$ (full line) and particles $v_{\mathrm{z}}$ (dotted line). To aid interpretation, we subtracted the mean velocity of the sedimenting particles $\left\langle v_{z}\right\rangle$ from the particle velocity and a small artificial upwards mean gas velocity $\left\langle u_{z}\right\rangle$ from the gas velocity. Displayed are different times $t=0,5,10 t_{\mathrm{f}}$, in respectively black, blue and red, based on the region between $z=0-10 l_{\mathrm{f}}$ in $r u n 2$. The standard deviations on the binned averages are relatively large, $\sigma \approx 0.03-0.04 v_{\mathrm{f}}$ for the gas and particle velocities between $t=5-10 t_{\mathrm{f}}$. The black dashed line corresponds to $\alpha=0.02$ in the toy model (Sec. 5.5.5). 
From inspection of our numerical results at different resolutions and spatial scales, we find that the instability tends to originate on the smallest available scale, near the grid scale in simulations with minimal viscosity (the dependency on the viscosity is discussed in Sec. 5.5.6 in more detail). This makes it computationally challenging to characterize the instability, as increased resolutions do not necessarily better resolve the characteristic scale of interest. Instead, the instability takes place a little faster and remains quantitatively similar (Fig. 5.2).

The view of the drafting instability as arising from an imbalanced pressure stratification suggests that the instability is non-linear, as the particle fluctuations that drive the horizontal imbalance must be seeded from the initial condition. This implies that the growth rate of the instability should decrease with increasing particle number, as randomly placed particles have a decreased effective density fluctuation with increased particle number. We show in Appendix 5.11 that indeed the fastest growth is found when reducing the particle number to just four per cell. However, we are not able to completely shut off the instability at larger particle number, indicating that the instability may operate even in the limit of very high particle number.

In the next sections we investigate how the sedimenting particles react to different perturbations of the system in order to gain further insight in the non-linear phase. We then propose that a toy model that can capture the dependency of the instability on the metallicity and Reynolds number (Sec. 5.5.5 - 5.5.6).

\subsubsection{Wave perturbation}

We now verify numerically that the drafting instability is not related to either the RayleighTaylor instability or the Kelvin-Helmholtz instability by perturbing the system with either a purely vertical or purely horizontal a vertical mode in the particle distribution.

We first present results of vertical wave perturbation of the particle density, which can be seen in Fig. 5.4. Within the time that particles sediment out of the domain, no instabilities can 
be detected. The right panel of Fig. 5.4 shows that the particles simply sediment at terminal velocity. At the same time, the gas component does not react to the perturbation. We have verified that this result even stands when feeding additional particle noise to the simulation. We do not see any Rayleigh-Taylor-like instabilities (that occur in a hydrostatic fluid with a dense layer on a lighter one, Drazin \& Reid 2004). This experiment also demonstrates that the mechanism concentrating particles is at least two-dimensional. In 1D simulations with only a vertical perturbation the ridges of increased particle density do not approach each other.

We also experiment with a horizontal wave in the particle distribution. To perturb the interface between the particle rich and poor region we displace the particle initially by giving them a random velocity kick of $v=0.1 v_{\mathrm{f}}$. The resulting evolution is shown in Fig. 5.5. The particle dense columns supersediment at an accelerated rate. This differential velocity between particle-poor and particle-rich columns drive an instability reminiscent of the KelvinHelmholtz instability (Drazin \& Reid 2004). However, in this case the denser fluid that is used in the classical description of the instability is replaced with a fluid containing an overdensity in particles. Such particle-loaded Kelvin-Helmholtz instabilities have been studied before in the context of molecular clouds (Hendrix \& Keppens 2014). A characteristic feature of the Kelvin-Helmholtz instability is the emergence of v-shaped wings. These can be identified in the bottom panel of Fig. 5.5. Similar features are also seen in simulations of the early linear evolution of the streaming instability in unstratified discs (see for example Fig. 2 in Johansen \& Youdin 2007). Therefore these wings might be a general feature of particle-gas instabilities.

We expect that this parasitic Kelvin-Helmholtz instability operates in the fully mixed state of our noise simulations when dense regions start sedimenting out. It may thus play an important role in the late non-linear evolution. However, since we only see this type of behaviour resembling Kelvin-Helmholtz instabilities in the case of a large perturbation, we 
do not believe it is the origin of the instability in the initial noise runs. 

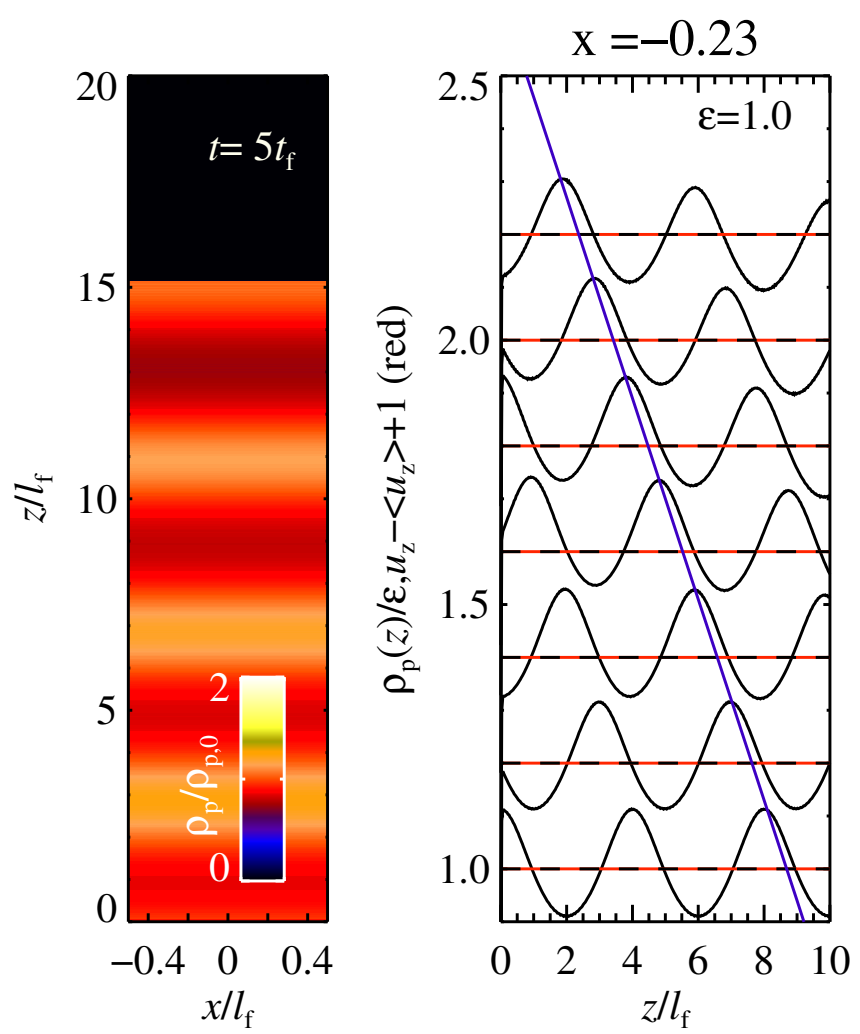

Figure 5.4.: Left panel: Sedimenting particles with a $k_{\mathrm{z}}=\pi / 2$-mode in the particle density. Represented is a snapshot of runRT at $t=5 t_{\mathrm{f}}$. No instabilities develop, suggesting that the drafting instability is not directly related to the Rayleigh-Taylor instabilities that should occur in this set up. Right panel: Evolution of the sedimenting particle wave at an arbitrary placed slice at $x=-0.23$. Different curves represent different times $\left(t=0,1,2, \ldots, 6 t_{\mathrm{f}}\right)$, that for clarity are offset by 0.2 . The particle density $\rho_{\mathrm{p}}(z)$ is given in black, while the red dashed line gives the gas velocity $u_{z}(z)$. Particle ridges do not approach each other, as indicated by the blue line that tracks the position of a point advected with velocity $v_{\mathrm{f}}$. The pressure that is supporting the mass-loaded stratification adapts to compensate for small changes in the particle density. This allows $u_{z}(z)$ to remain zero while the particles sediment. 

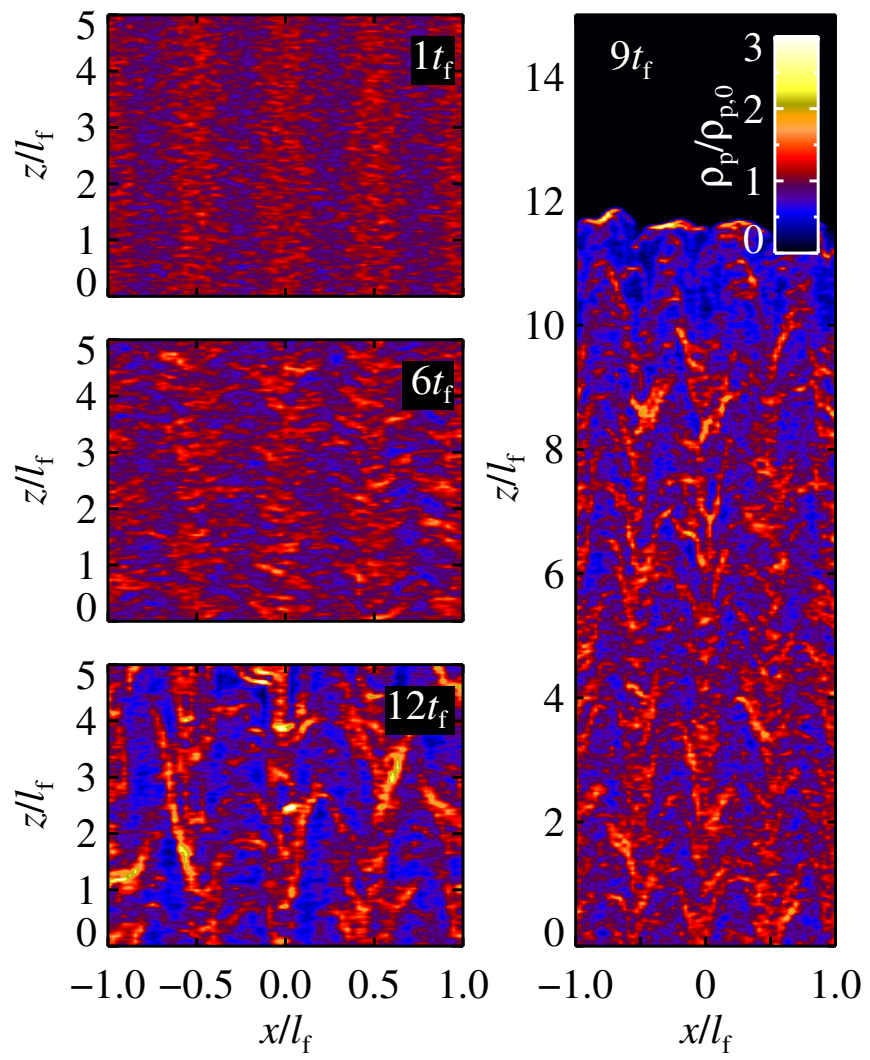

Figure 5.5.: Sedimenting particles with a $k_{\mathrm{x}}=\pi / 2$-mode in the particle density. We show different details (left) from a larger simulated domain (right), at different times $(t=$ $1,6,9,12 t_{\mathrm{f}}$ ), based on runKH. We added initially noise in the particle velocity perturb the boundary between particle rich and poor. We believe that we see a "parasitic" Kelvin-Helmholtz instability appear over time. 


\subsubsection{Eggbox perturbation}

Finally, we also perturb the system with an eggbox-like perturbation, in order to investigate the formation and evolution of particle swarms, which we will term particle droplets. The initial particle density perturbation is of the form

$$
\rho_{\mathrm{p}}(x, z)=A \sin \left(k_{\mathrm{x}} x\right) \sin \left(k_{\mathrm{z}} z\right)
$$

with $A$ the amplitude of the two-dimensional perturbation. This initial condition can be inspected in Fig.5.6. The subsequent panels show the evolution of the inserted particle droplets. Initially they go through a phase of contraction without altering the amplitude. This can be seen in further detail in the vertical slices in Fig.5.7. The droplets remain in terminal velocity. Intriguingly, the wave steepening is scale-independent, as can be seen the right panel of Fig.5.7, where we have decreased the size of the droplets by a factor 2 . Subsequently, the particle transforms in a characteristic mushroom cloud reminiscent of those seen in the standard Rayleigh-Taylor instability.

Figure 5.8 illustrates how non-linear drafting results in particle concentrations. Initially, the droplets concentrate the material by collapsing onto themselves. However, in the subsequent evolution it is clear that the overdense regions drag gas downwards with their sedimentation flow. Simultaneously, gas moves upwards in between the denser areas. This is an aspect of the unbalanced stratification that we discussed in Sec. 5.5.2.

\subsubsection{Toy model of the drafting instability}

The origin of the instability can be grasped from a simplified stability analysis, that is based on the observation that regions overdense in particles sediment faster than regions that are underdense in particles. Eq. (5.5) and Eq. (5.6) describe the behaviour of the particles, which only depends on the gas through the gas drag term. We now assume the gas velocity can be 


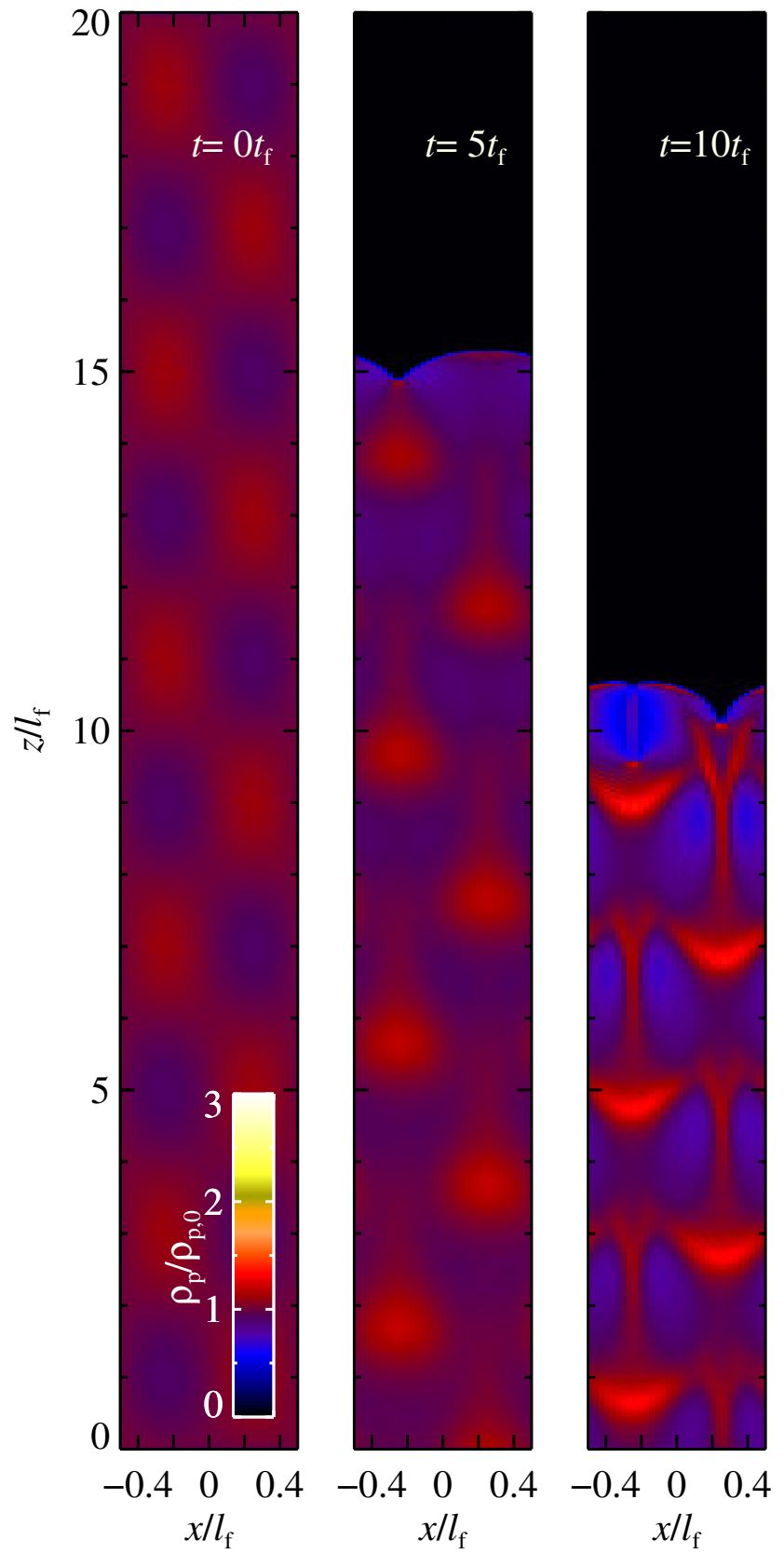

Figure 5.6.: The evolution of particle droplets, at different times $\left(t=0,5,10 t_{\mathrm{f}}\right)$, resulting in the emergence of characteristic Rayleigh-Taylor mushroom clouds. The colorbar give the color scale for the shown particle density. Results from runEGG. 


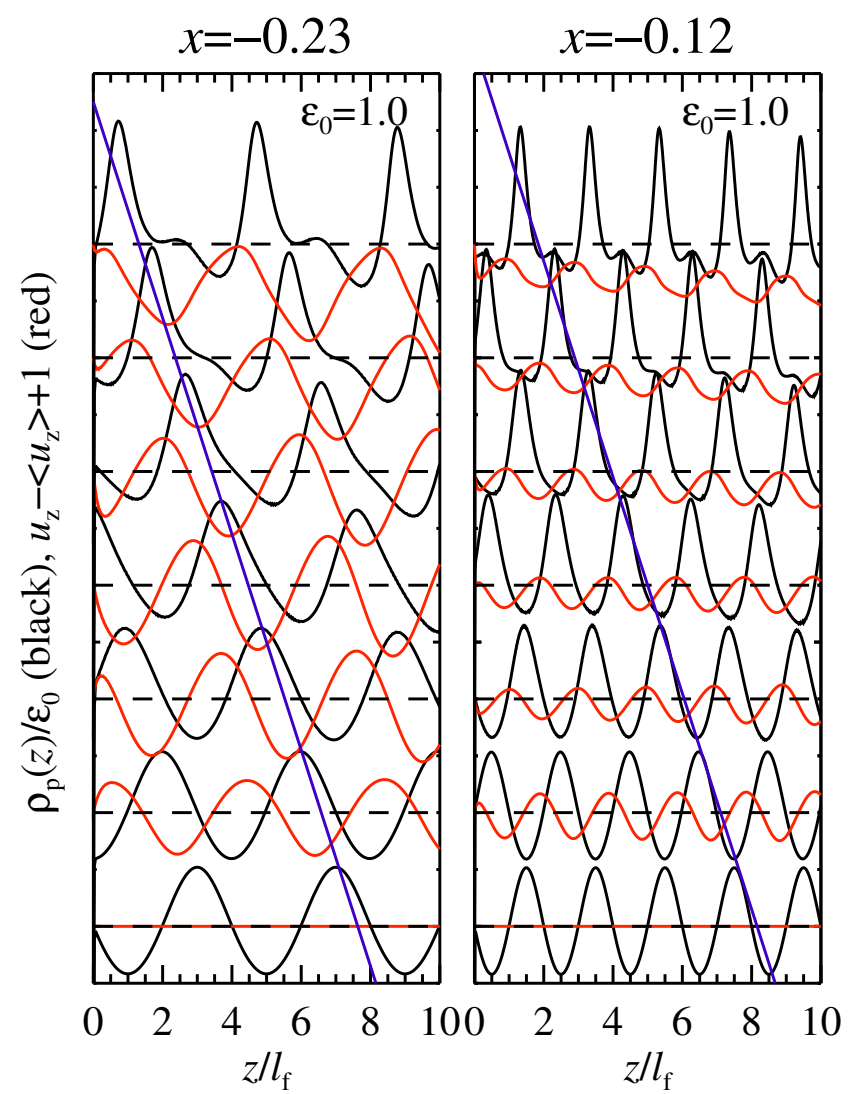

Figure 5.7.: Slices through the evolution of the particle droplets, along the $z$-direction, similar to Fig.5.4. The droplets sediment at terminal velocity, and the wave steepening is independent of the initial droplet scale. The left panel shows run runEGG with $\lambda_{\mathrm{x}}=1, \lambda_{\mathrm{z}}=4$ and the right panel runEGG2 with $\lambda_{\mathrm{x}}=0.5, \lambda_{\mathrm{z}}=2$.

written as

$$
u=\alpha\left(\varepsilon-\varepsilon_{0}\right) v
$$

Here, $\alpha$ is a proportionality constant that can be determined numerically and which contains the dependence of the instability on the viscosity and the Mach number. The quantities $u$ and $v$ are the vertical gas and particle velocity. Effectively, we use that the linearised gas velocity $\delta u$ can be expressed proportional to the particle density perturbation $\delta u=\alpha\left(v_{\mathrm{f}} / \rho_{0}\right) \delta \rho_{\mathrm{p}}$ (see Appendix 5.10 for more details). Physically, it expresses the observation that gas follows 

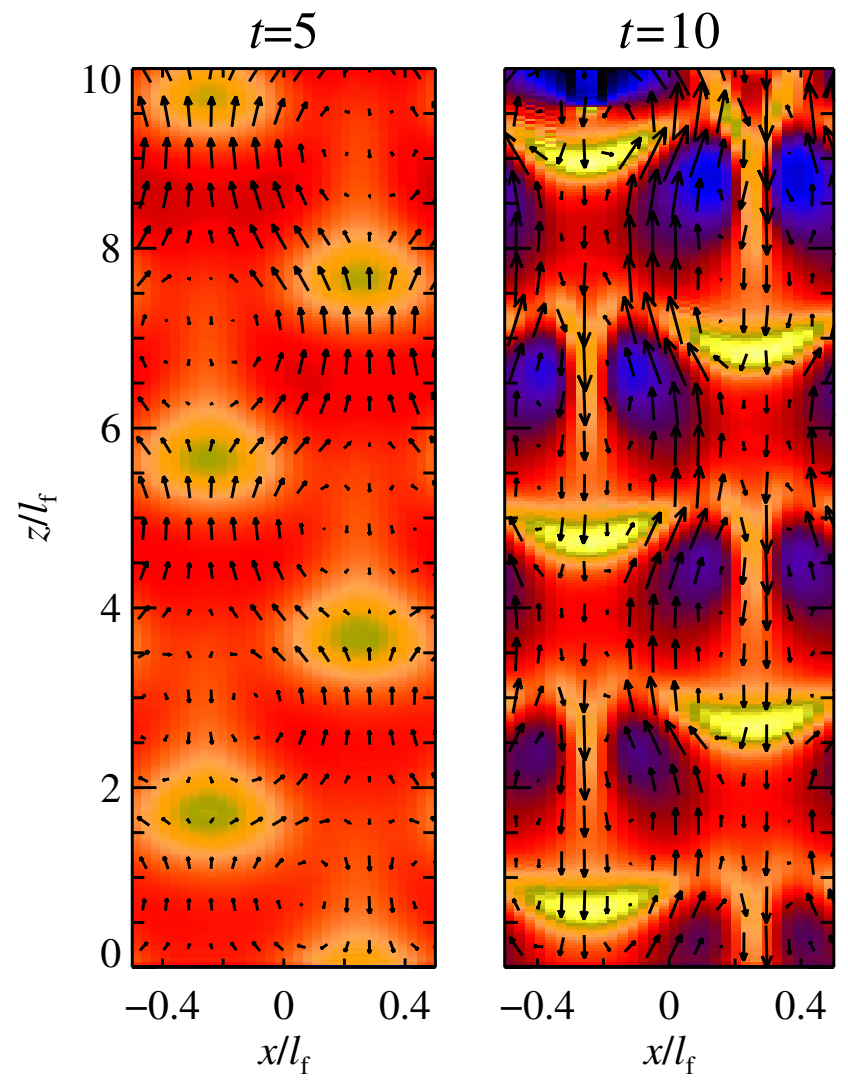

Figure 5.8.: The particle and gas evolution around particle droplets, at times $t=5,10 t_{\mathrm{f}}$. Streamlines show the gas velocity, while the particle density is color coded as in Fig. 5.6. Results from runEGG.

overdense particle regions, but locally momentum is conserved by the gas becoming buoyant and moving in the opposite direction in underdense regions.

This assumption allows us to reduce the equations to one dimension, even though the above approximation implicitly assumes two or three dimensions to be present to allow gas to move freely and not be trapped as in our stable one dimensional experiment (Fig.5.8). We will also approximate the gas density to be constant.

The equilibrium state corresponds to pure sedimentation with $\varepsilon=\varepsilon_{0}$ and the particles having the terminal velocity $v=-g t_{\mathrm{f}}$. The dispersion relation for Fourier modes of the form 
$\propto \exp (\omega t-i k x)$ becomes

$$
\omega^{\prime}=+i k^{\prime}+\frac{1}{2}\left(-1 \pm \sqrt{1-4 \alpha \varepsilon_{0} k^{\prime} i}\right)
$$

where $\omega^{\prime}$ is the growth amplitude, $k^{\prime}=2 \pi / \lambda^{\prime}$ the wave number of wavelength $\lambda^{\prime}$ in friction units. The last term of Eq. (5.10) is always positive ${ }^{3}$ for $\varepsilon_{0}>0$, resulting in the exponential growth of the instability. The fastest growing modes are those with the shortest wavelength. This result, although derived somewhat differently (see Appendix 5.10), is identical to that of plate drag (Goodman \& Pindor 2000, Chiang \& Youdin 2010, Jacquet et al. 2011). The real part of the dispersion relation is illustrated in Fig. 5.12.

For large $k$, corresponding to short wavelengths $\left(\lambda \ll 8 \pi \alpha \varepsilon_{0} t_{f}^{2} g\right)$, the growth rate can be approximated by

$$
\omega_{\text {grow }}^{\prime} \approx \frac{1}{\sqrt{2}} \sqrt{\alpha \varepsilon_{0} k^{\prime}}
$$

by series expansion to leading order. Alternatively, we can express this result no longer in friction units but as

$$
\omega_{\text {grow }} \approx \sqrt{\alpha \pi \frac{\varepsilon_{0} g}{\lambda}}
$$

Interestingly, in this asymptotic limit case the growth rate no longer depends on the particle size (or more accurately the friction time), but only on the spatial scale and the dust-to-gas ratio. On larger scales, the limit expression of the growth rate takes the form

$$
\omega_{\text {grow }}^{\prime}=\left(\alpha \varepsilon_{0} k^{\prime}\right)^{2},
$$

\footnotetext{
${ }^{3}$ The real part of $\sqrt{1-i x}$ is always larger than 1 .
} 
to leading order. Therefore, the growth rate of the drafting instability rapidly decreases with increased spatial scale. In this branch the growth rate does depend on the particle size,

$$
\omega_{\text {grow }}=(\alpha \varepsilon k g)^{2} t_{\mathrm{f}}^{3}
$$

\subsubsection{Dependence on the Reynolds number}

If the toy model is right, then the growth rate scales as $\omega_{\text {grow }}^{\prime} \propto k^{\prime 2}$ on length scales above the characteristic scale

$$
\lambda_{\text {knee }}=2^{4 / 3} \pi \alpha \varepsilon_{0} l_{\mathrm{f}}
$$

obtained from balancing the fast and slow growth branches (Eq. 5.11 and 5.13).

Viscous damping has a similar quadratic dependence on $k^{\prime}$. Therefore a viscosity cut-off exists: at $v$ larger than $v_{\text {crit }}$ growth of the instability is terminated. We find the critical viscosity by equating the large scale growth time scale (Eq. 5.13) with the viscous time scale $\left(\lambda^{\prime 2} / v^{\prime}\right)$

$$
v_{\text {crit }}^{\prime}=2 \pi(\alpha \varepsilon)^{2}
$$

The determination of $\alpha$ allows us to scale the growth rates of the toy model. From Fig. 5.9 we find numerically that above viscosities of around $v^{\prime} \sim 10^{-3}$, the instability does not show up. A critical viscosity of $v_{\text {crit }}^{\prime} \sim 10^{-3}$ would correspond to $\alpha \sim 10^{-2}$. Such an estimate is an approximate agreement with the seen correlation between particle density variations $\varepsilon$ and the gas fluid velocity in Fig. 5.3.

For viscosities below the viscosity cut-off on the small scale branch, the the largest growing 


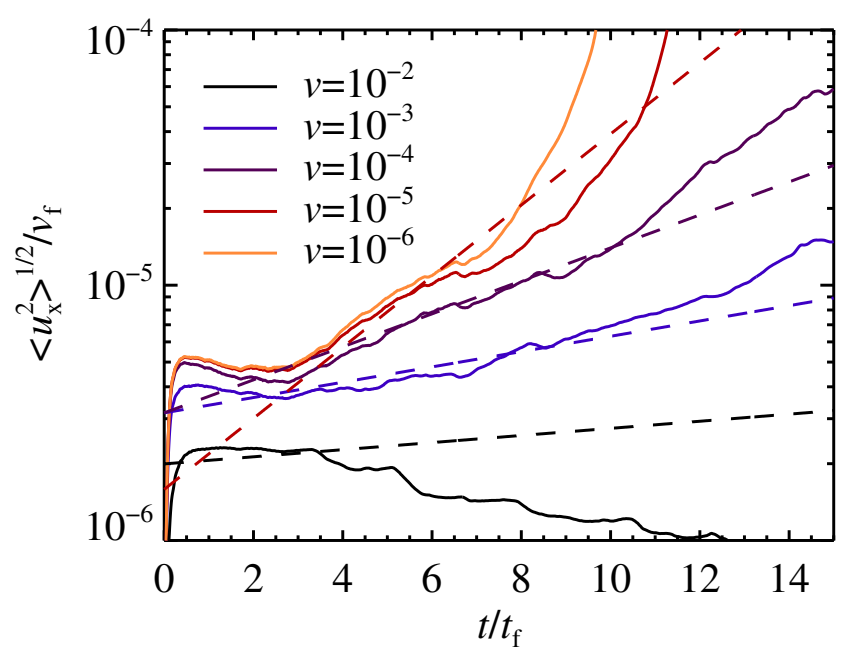

Figure 5.9.: Viscosity dependency on the growth rate of the drafting instability. Dashed lines give the growth time scaling where $\omega \propto v^{-1 / 3}$, as in the toy model. At high viscosities over $v^{\prime} \approx 10^{-3}$ we no longer identify growth in the fluid velocity dispersion. Numerical results from runv2, runv3, run2, runv5, runv6.

wavenumber scales as

$$
k_{\text {crit }}^{\prime}=\left(2 \pi^{2} \frac{\alpha \varepsilon_{0}}{v^{\prime 2}}\right)^{1 / 3}
$$

by setting the viscous time scale equal to small scale growth rate (Eq. 5.11). Therefore the growth rate scales with the viscosity as $\omega^{\prime} \propto v^{\prime-1 / 3}$. This indeed agrees with the results shown in Fig. 5.9.

\subsubsection{Dependence on initial dust-to-gas ratio}

The main free parameter in our model is the initial dust-to-gas ratio, also called the metallicity, when setting up the equilibrium stratification $\varepsilon_{0}$. Evidently in the limit of negligible dust loading, we do not expect any dust clumping. We therefore study the dependency of the growth rate of the instability on lower than unity initial dust-to-gas ratio (Fig.5.10). To measure slower growth rates (and possibly the saturation of the instability), we need to 
extend the vertical domain, which we achieve by numerically scaling the system (runs run $1-4 \cdot 0.1$, see Table 5.2).

We find that the instability does not vanish even at a 10 times reduced metallicity. The growth rate is slower, and there seems to be a longer dormant phase before particle concentrations settle in. The reason of this delay for the instability to kick in is unclear. Between $\varepsilon_{0}=0.1$ to $\varepsilon_{0}=1$ the growth rates scale approximately proportionally to $\sqrt{\varepsilon_{0}}$, as expected form the toy model (Eq. 5.12).

At even lower metallicities, $\varepsilon_{0}=0.05$ we do not recover the instability. Growth rates become too slow to identify any particle clumping in the simulation (run 4.01 ).
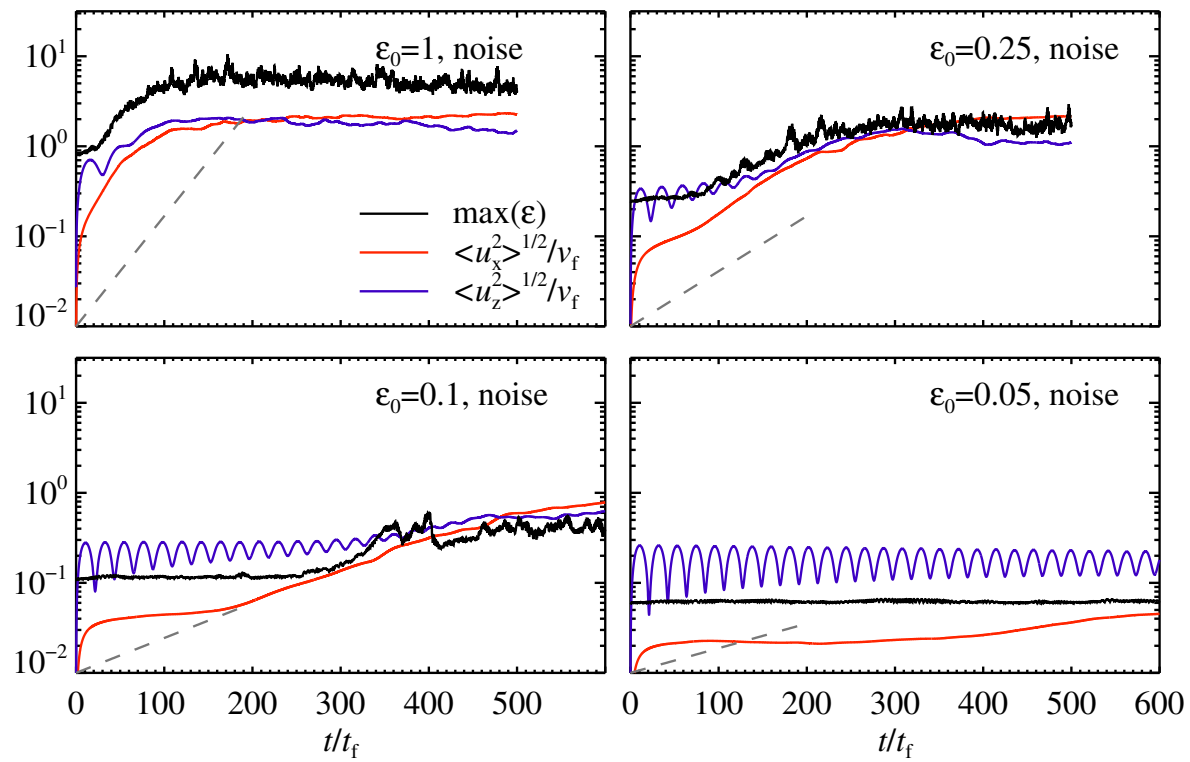

Figure 5.10.: Long term evolution of the dust-to-gas ratio $\max (\varepsilon)$ (black), combined with the horizontal $\sqrt{\left\langle u_{x}^{2}\right\rangle}$ (red) and vertical $\sqrt{\left\langle u_{z}^{2}\right\rangle}$ (blue). The grey dashed lines represent the growth rate scaling with $\sqrt{\varepsilon_{0}}$, as found from the toy model (Eq. 5.11). The growth rate decreases with dust-to-gas ratio, and we are unable to measure growth rates below $\varepsilon_{0}=0.05$. The small oscillations seen in vertical velocity dispersion for the low metallicity runs are the result of vertical waves which are due to a slight imbalance in the initial condition in elongated domains. The figure is based on the simulations run1.01, run2.01, run3.01, run 4.01 (in order of decreasing metallicity). 


\subsection{Enhanced particle concentrations in protoplanetary discs}

In this section, we rescale our simulation results to the context of the protoplanetary disc. Because the drafting effect seems to prefer higher dust-to-gas ratios than the percentage level initially expected in a protoplanetary disc, we will consider particle settling in a disc that has already undergone some grain growth, resulting in an already partly settled particle midplane (Sec. 5.6.1 and 5.6.2). In mass loaded regions, the swarms created by the drating instability may aid the formation of chondrules, which we explore in Sec. 5.6.3. Finally we comment on the relevance of the drafting instability in the possibly highly dust-enriched envelopes around protoplanets (Sec. 5.6.4).

\subsubsection{Rescaling friction units}

The friction units employed in our simulations can be readily rescaled to a protoplanetary disc setting, for a given particle size expressed in Stokes number

$$
\tau_{\mathrm{f}}=t_{\mathrm{f}} \Omega_{\mathrm{K}}
$$

where $\Omega_{\mathrm{K}}$ is the Keplerian frequency (for the definition of the friction time $t_{\mathrm{f}}$, see Eq. 5.3). From this definition, a time $t_{\mathrm{f}}$ corresponds to a fraction of a Keplerian time scale, $t_{\mathrm{f}}=\Omega_{\mathrm{K}}^{-1} \tau_{\mathrm{f}}$ . Similarly, the friction length $l_{\mathrm{f}}$ can be expressed as

$$
l_{\mathrm{f}}=g t_{\mathrm{f}}^{2}=z \tau_{\mathrm{f}}^{2},
$$

when the gravity is expressed as $\Omega^{2} z$. Here, $z$ is the height above the midplane. The friction length in the Minimum Mass Solar Nebula (MMSN, Hayashi 1981) at the top of a particle 
layer of thickness $H_{\mathrm{p}}$ can be written as

$$
l_{\mathrm{f}} \approx 37 \times 10^{3}\left(\frac{H_{\mathrm{p}} / H}{0.1}\right)\left(\frac{\tau_{\mathrm{f}}}{0.1}\right)^{2}\left(\frac{r}{5 \mathrm{AU}}\right)^{5 / 4} \mathrm{~km} .
$$

This scale strongly depends on the particle size $\left(\tau_{\mathrm{f}}=0.1\right.$ corresponds to a $2 \mathrm{~cm}$ particle at an orbital distance of $r \approx 5 \mathrm{AU}$ ). We have here assumed that the particle scale height is a constant fraction of the gas scale height $H$.

The ratio of the terminal velocity to the sound speed, the Mach number

$$
M a=\frac{v_{\mathrm{f}}}{c_{\mathrm{s}}}=0.01\left(\frac{H_{\mathrm{p}} / H}{0.1}\right)\left(\frac{\tau_{\mathrm{f}}}{0.1}\right)
$$

reveals the incompressible nature of particle sedimentation.

We can ignore the overall rotation of the protoplanetary disc for the small scales that we consider here. The Rossby number $R o=v_{\mathrm{f}} /\left(\Omega_{\mathrm{K}} l_{\mathrm{f}}\right)$ takes the form: $R o \sim 1 /\left(\Omega_{\mathrm{K}} t_{\mathrm{f}}\right)$, when using friction scales. Therefore, for particles with small Stokes number $\tau_{\mathrm{f}}=\Omega_{\mathrm{K}} t_{\mathrm{f}} \ll 1$, rotation is not important, and the rotation-free assumption is valid.

The kinematic molecular viscosity depends on the gas mean free path $\lambda$ in the midplane of the protoplanetary disc as

$$
v=\frac{1}{2} c_{\mathrm{s}} \lambda
$$

The viscosity can then be expressed in friction units as

$$
\frac{v}{g^{2} t_{\mathrm{f}}^{3}}=1.6 \times 10^{-6}\left(\frac{H_{\mathrm{p}} / H}{0.1}\right)^{-2}\left(\frac{\tau_{\mathrm{f}}}{0.1}\right)^{-3}\left(\frac{r}{5 \mathrm{AU}}\right)^{3 / 2}
$$

This value does not differ greatly from the nominal value probed in our numerical work $\left[v /\left(g^{2} t_{\mathrm{f}}^{3}\right)=10^{-4}\right.$, see also the list of simulations in Table 5.1]. The strong scaling with 
orbital radius becomes much weaker if one considers particles of constant radius, as opposed to constant Stokes number.

Finally, we also verify the viscous particle coupling criterion, given by Eq. (5.4), holds in the MMSN,

$$
\frac{t_{v, \text { pair }}}{t_{\mathrm{f}}} \approx 3.0 \times 10^{-4}\left(\frac{\varepsilon_{0}}{0.1}\right)^{-2 / 3}\left(\frac{\tau_{\mathrm{f}}}{0.1}\right)\left(\frac{r}{5 \mathrm{AU}}\right)^{-31 / 6},
$$

where $\varepsilon_{0}$ is the approximate mean dust-to-gas ratio in the particle midplane.

\subsubsection{Applying the toy model}

With the help of the toy model we can attempt to further constrain where in the protoplanetary disc the drafting instability can occur. Because growth rates decrease rapidly at large scales, we only expect the instability to take place on the small scale branch, below the characteristic scale $\lambda_{\text {knee. }}$. From Eq. 5.15, we get

$$
\lambda_{\text {knee }} \approx 290\left(\frac{\alpha}{0.01}\right)\left(\frac{\varepsilon_{0}}{0.1}\right)\left(\frac{H_{\mathrm{p}} / H}{0.1}\right)\left(\frac{\tau_{\mathrm{f}}}{0.1}\right)^{2}\left(\frac{r}{5 \mathrm{AU}}\right)^{5 / 4} \mathrm{~km} .
$$

The dust-to-gas ratio of $\varepsilon_{0}=0.1$ will be relevant in a midplane layer of solids with $H_{p} / H=0.1$, when the overall metallicity of the protoplanetary disc is the canonical $Z=0.01$. However, we have chosen to keep dust-to-gas ratio $\varepsilon_{0}$ and the height of the particle layer $H_{\mathrm{p}}$ as independent quantities, because we do not necessarily want to study the conditions of a particle layer settled to equilibrium.

A lower limit on the scale of the instability is set by viscous damping of the instability at 
scales below the knee,

$$
\begin{aligned}
\lambda_{\text {visc }} & =\left(\frac{2^{2} \pi}{\alpha \varepsilon_{0}}\right)^{1 / 3}\left(\frac{v}{g^{2} t_{\mathrm{f}}^{3}}\right)^{2 / 3} l_{\mathrm{f}} \\
& \approx 93\left(\frac{\alpha}{0.01}\right)^{-1 / 3}\left(\frac{\varepsilon_{0}}{0.1}\right)^{-1 / 3}\left(\frac{H_{\mathrm{p}} / H}{0.1}\right)^{-1 / 3}\left(\frac{r}{5 \mathrm{AU}}\right)^{9 / 4} \mathrm{~km} .
\end{aligned}
$$

Note this scale, as opposed to the friction length $l_{\mathrm{f}}$ (Eq. 5.20), does not depend on the particle size.

In Fig. 5.11 we have illustrated the different relevant scales presented in Eq. (5.20), (5.25) and (5.26), as function of orbital radius. The instability would operate on a scale of the order of $10^{4} \mathrm{~km}$ in the outer parts of the protoplanetary disc for particles of $\mathrm{cm}$ in size, assuming a MMSN model. Note that Fig. 5.11 shows the scaling for an assumed constant particle size, as opposed to Eq. (5.20-5.26) that assume constant Stokes number.

\subsubsection{Chondrules}

Chondrules are mm-sized inclusions found in primitive meteorites originating from the asteroid belt. It is generally accepted that a chondrule is the product of a flash heating event. The exact nature of chondrule precursors is unknown. However the heating events likely occurred in particle swarms at least 100 to $1000 \mathrm{~km}$ wide, with a local number density of about $\sim 10 \mathrm{~m}^{-3}$. In this way the loss of light isotopes (isotopic fractionation) is prevented by exchanging vapour from chondrule to chondrule (Cuzzi \& Alexander 2006). This scenario requires local chondrule densities more than 100 times above a dust-to-gas ratio of unity. Even higher concentrations might be necessary to explain the retention of sodium (Alexander et al. 2008).

Such high chondrule densities are surprising, since small particles are hard to concentrate to the midplane. Even in the absence of other forms of turbulence, particles sediment to a 
midplane with dust-to-gas ratio not higher than approximately unity, because of the stirring caused by the streaming instability (Bai \& Stone 2010b). However, the isotopic constraints on the need to concentrate chondrules weaken if the gas at the chondrule formation sites had a non-solar composition. The atmospheres around planetary embryos have been proposed to be such locations (Morris et al. 2012). Nevertheless, in this scenario, pre-clumping of solids by a factor of at least 10 over midplane densities remains necessary and the shock waves invoked to melt chondrules lead in fact to destructive collisions (Jacquet \& Thompson 2014).

Small particles are difficult to concentrate in the inner protoplanetary disc, because of the strong sensitivity of the preferential scale of the instability on particle size (Eq. 5.20 and Eq. 5.25), as can be seen in Fig. 5.11. Nevertheless the connection to chondrule formation is tantalizing, especially because if clumping conditions are met, the drafting effect only weakly depends on particle size and efficiently clusters particles down to very small sizes (Eq. 5.12). This is different from, for example, the streaming instability that has a preferred particle size, somewhat above that of chondrules for nominal metallicities (Carrera et al. 2015).

The drafting instability could operate on such small scales, if some form of pre-concentration of solids would occur. Possibly such enhanced particle densities could occur near the Kolmogorov scale of the disc turbulence (Cuzzi et al. 2001b). Alternatively, near sublimation lines particle concentrations can dramatically peak (Ros \& Johansen 2013). An increase in the dust-to-gas ratio can also occur by accretion of gas onto the star, which depletes the disc relative to the MMSN (Bitsch et al. 2015). Alternatively, growth rates could be increased if the unknown chondrule precursors are much larger than the chondrules they are turned into after the heating event. Even so, it remains to be seen if drafting instabilities can push particle concentrations to the desired high levels, even in such favourable instances. 


\subsubsection{Planetary atmospheres}

The drafting instability might be important in the atmospheres of giant planets. The opacity in the outer envelope, which regulates the transport of heat, comes from the dust component. Under standardly assumed opacities, it is difficult to cool the envelope and trigger runaway gas accretion (Ikoma et al. 2000, Piso \& Youdin 2014). However, clumping of solids and the growth of the accreted dust could significantly reduce the opacity in the upper atmosphere. The friction length for particles sedimenting in a planetary atmosphere is given by

$$
\begin{aligned}
l_{\mathrm{f}, \mathrm{plan}} & =\frac{G M}{r_{\mathrm{B}}^{2}} t_{\mathrm{f}}^{2} \\
& \approx 7.2 \times 10^{3}\left(\frac{R}{1 \mathrm{~mm}}\right)^{2}\left(\frac{M}{5 \mathrm{M}_{\mathrm{E}}}\right)^{-1}\left(\frac{r}{5 \mathrm{AU}}\right)^{5} \mathrm{~km},
\end{aligned}
$$

where $r_{\mathrm{B}}=G M / c_{\mathrm{s}}^{2}$ is the thermal Bondi radius of a planet with mass $M$, corresponding to the outer edge of the atmosphere. We have here considered particles on top of the envelope, but deeper in the planet the friction time shrinks due to the increase in density. Applying the toy model, we estimate the knee scale in the upper envelope at

$$
\lambda_{\text {knee,plan }} \approx 570\left(\frac{\alpha}{0.01}\right)\left(\frac{\varepsilon_{0}}{1}\right)\left(\frac{R}{1 \mathrm{~mm}}\right)^{2}\left(\frac{M}{5 \mathrm{M}_{\mathrm{E}}}\right)^{-1}\left(\frac{r}{5 \mathrm{AU}}\right)^{5} \mathrm{~km}
$$

which is above the damping viscosity scale at

$$
\lambda_{\text {visc,plan }} \approx 13\left(\frac{\alpha}{0.01}\right)^{-1 / 3}\left(\frac{\varepsilon_{0}}{1}\right)^{-1 / 3}\left(\frac{M}{5 \mathrm{M}_{\mathrm{E}}}\right)^{1 / 3}\left(\frac{r}{5 \mathrm{AU}}\right)^{2} \mathrm{~km} .
$$

The formation of ice giants and super-Earths might be paired with significant amounts of dust in their low-mass gaseous envelopes (Lee et al. 2014). These scaling relations argue that order-of-unity mass loading of atmospheres will lead to clumping and the breakup of the dust component, providing an upper limit on the dust opacity. 


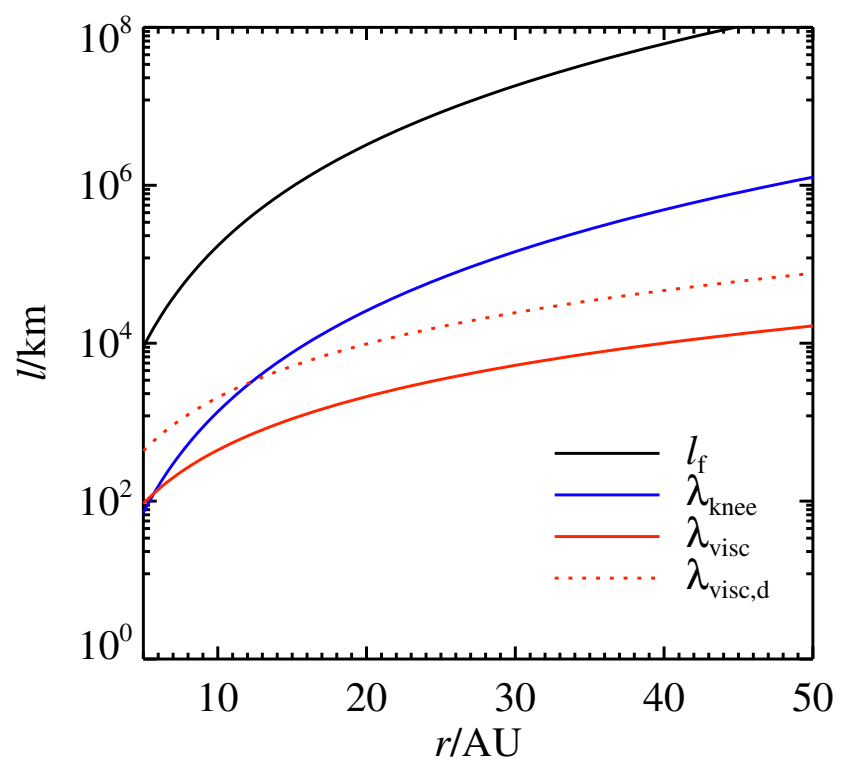

Figure 5.11.: Relevant length scales for the drafting instability in the Minimum Mass Solar Nebula: the friction length $\left(l_{\mathrm{f}}\right)$, the upper scale for fast growth $\left(\lambda_{\text {knee }}\right)$, and the scale at which viscosity dominates $\left(\lambda_{\text {visc }}\right)$. We consider particles located at a particle scale height above the midplane, with $H_{\mathrm{p}} / H=0.1$ and the midplane dust-to-gas ratio is 0.1 . We take the toy model parameter to be $\alpha=0.01$. Particles are assumed to be $1 \mathrm{~cm}$ in radius, or $1 \mathrm{~mm}$ in a gas depleted disc with 10 times lower gas surface density (in that case the curves remain the same, but viscous scale $\lambda_{\text {visc,d }}$ is now the red dotted line). Likely the instability does not operate in the inner $(<5 \mathrm{AU})$ of the protoplanetary disc, unless particles are large or significant pre-concentration occurs.

\subsection{Future outlook}

[Section omitted, moved to Chapter Two.]

\subsubsection{Numerical work}

We have here presented several numeric experiments to demonstrate a drafting instability.

Future work will refine the estimates made in this paper.

For example, currently the numerical set up is limited to studying sedimentation on rather short timescales, set by the length of the simulation domain. This could be avoided in future work by implementing a form of periodic boundary conditions in the vertical direction, which 
would recycle particles.

To aid the interpretation of the experimental results, it will be necessary to specifically reproduce the parameter regime in which the apparatus operates. Additionally, refined boundary conditions will be needed to approximate the experimental set-up. Such work is under progress, but evidently awaits the first experiments.

We have also argued that the drafting instability could be of relevance in a protoplanetary disc. To study this connection in more detail, it will be necessary to simulate numerically expensive larger domains encompassing the disc midplane. Additionally, the connection between the drafting and streaming instability could be studied in more detail. Ultimately, the results should be placed in the context of other sources of disc turbulence, such as the magnetorotational instability operating in sufficiently ionised regions or in the penetration of vertical shear instability to the midplane (Turner et al. 2014). Additionally, the growth of particles through coagulation or condensation will need to be taken into account in a selfconsistent matter. Future work is needed to understand the possibly constructive interplay of these mechanisms.

\subsection{Summary}

In this paper we have demonstrated the presence of a drafting instability when particles sediment through a fluid in hydrostatic balance. On time scales of tens of friction times particle unmix out of a homogeneous mixture and particle concentrations increase by a factor 10.

The presence of such an instability was not expected because it evades detection in an analytic linear stability analysis. However, our numerical results demonstrate that the system is non-linearly unstable. The exact nature of the instability is difficult to determine. We interpret the instability to be the result of an imbalance in the stratification locally disturbing 
the hydrostatic balance. We support this hypothesis with a simple toy model that captures some of the main characteristics of the instability. Growth is fastest at the smallest available scales, increases with the square root of the dust-to-gas ratio and a critical scale is identified at which viscosity overwhelms the instability.

By expressing our numerical results in a system of friction units, we can exploit our results by scaling them to either upcoming laboratory experiments or the protoplanetary disc. We argue that an experiment can probe a similar regime with dust-to-gas ratio around unity that is of interest here. In protoplanetary discs the drafting instability may take place in particle-rich layers above the midplane in the outer regions of the disc, on scales smaller than previously studied. In these regions where the conditions for the drafting instability are met, we have shown that sedimenting particles spontaneously form dense clumps. Future work will be needed to investigate to what degree this clumping affects coagulation rates and whether the drafting instability can create the dense environment necessary for chondrule flash heating.

\subsection{Linear stability analysis}

We briefly rederive the stability analysis for a particle gas-mixture in a non-rotating flow (Youdin \& Goodman 2005, Jacquet et al. 2011). We demonstrate the result in two spatial dimensions, but our conclusions remain valid when generalized to three dimensions.

\subsubsection{Governing equations}

We assume the gas to be incompressible, in line with Youdin \& Goodman (2005),

$$
\frac{\partial u_{x}}{\partial x}+\frac{\partial u_{z}}{\partial z}=0
$$


and use the standard momentum equations

$$
\begin{aligned}
& \frac{\partial u_{x}}{\partial t}+u_{x} \frac{\partial u_{x}}{\partial x}+u_{z} \frac{\partial u_{x}}{\partial z}=-\frac{1}{\rho} \frac{\partial P}{\partial x}+\frac{1}{t_{\mathrm{f}}} \frac{\rho_{\mathrm{p}}}{\rho}\left(v_{x}-u_{x}\right), \\
& \frac{\partial u_{z}}{\partial t}+u_{x} \frac{\partial u_{z}}{\partial x}+u_{z} \frac{\partial u_{z}}{\partial z}=-g-\frac{1}{\rho} \frac{\partial P}{\partial z}+\frac{1}{t_{\mathrm{f}}} \frac{\rho_{\mathrm{p}}}{\rho}\left(v_{z}-u_{z}\right) .
\end{aligned}
$$

Similarly, for the particle fluid we make use of the continuity equation,

$$
\frac{\partial \rho_{\mathrm{p}}}{\partial t}+\frac{\partial}{\partial x}\left(\rho_{\mathrm{p}} v_{x}\right)+\frac{\partial}{\partial z}\left(\rho_{\mathrm{p}} v_{z}\right)=0
$$

and the set of momentum equations

$$
\begin{aligned}
& \frac{\partial v_{x}}{\partial t}+v_{x} \frac{\partial v_{x}}{\partial x}+v_{z} \frac{\partial v_{x}}{\partial z}=-\frac{1}{t_{\mathrm{f}}}\left(v_{x}-u_{x}\right) \\
& \frac{\partial v_{z}}{\partial t}+v_{x} \frac{\partial v_{z}}{\partial x}+v_{z} \frac{\partial v_{z}}{\partial z}=-g-\frac{1}{t_{\mathrm{f}}}\left(v_{z}-u_{z}\right) .
\end{aligned}
$$

This completes the model with 6 parameters $\left(\rho_{\mathrm{p}}, \rho, v_{x}, v_{z}, u_{x}, u_{z}\right)$, and as many equations.

\subsubsection{Equilibrium solution}

In equilibrium we have no vertical motion $\left(u_{x}=0, v_{x}=0\right)$. Additionally, we assume the gas to be in the rest frame, $u_{z}=0$, and particles to be initially uniformly spread ( $\rho_{\mathrm{p}}=\rho_{\mathrm{p}, 0}$ constant). This leaves the particle continuity and $z$-momentum equations as the non-trivial equations determining $v_{z}$ and $\rho, \rho_{\mathrm{p}}$,

$$
\begin{aligned}
\frac{\partial}{\partial z}\left(\rho_{\mathrm{p}} v_{z}\right) & =0, \\
v_{z} \frac{\partial v_{z}}{\partial z} & =-g-\frac{1}{t_{\mathrm{f}}} v_{z}, \\
0 & =-g-\frac{c_{\mathrm{s}}^{2}}{\rho} \frac{\partial \rho}{\partial z}+\frac{1}{t_{\mathrm{f}}} \frac{\rho_{\mathrm{p}}}{\rho} v_{z} .
\end{aligned}
$$


In the last equation we have assumed an isothermal gas, $P=\rho c_{\mathrm{s}}^{2}$. The equilibrium solution then takes the form

$$
\begin{aligned}
& v_{z}=-g t_{\mathrm{f}} \\
& \rho=\left(\rho_{\mathrm{p}}+\rho_{\mathrm{b}}\right) \exp \left(-\frac{g}{c_{\mathrm{s}}^{2}} z\right)-\rho_{\mathrm{p}},
\end{aligned}
$$

where $\rho_{\mathrm{b}}$ is the gas density at the $z=0$ boundary.

\subsubsection{Dispersion relation}

We now consider a first order perturbation of this equilibrium state. For the gas we find

$$
\begin{aligned}
\frac{\partial u_{x}^{\prime}}{\partial x}+\frac{\partial u_{z}^{\prime}}{\partial z} & =0, \\
\frac{\partial u_{x}^{\prime}}{\partial t} & =-\frac{c_{\mathrm{s}}^{2}}{\rho_{0}} \frac{\partial \rho^{\prime}}{\partial x}+\frac{1}{t_{\mathrm{f}}} \frac{\rho_{\mathrm{p}, 0}}{\rho_{0}}\left(v_{x}^{\prime}-u_{x}^{\prime}\right), \\
\frac{\partial u_{z}^{\prime}}{\partial t} & =-\frac{c_{\mathrm{s}}^{2}}{\rho_{0}} \frac{\partial \rho^{\prime}}{\partial z}-\frac{g}{\rho_{0}} \rho^{\prime}-\frac{g}{\rho_{0}} \rho_{p}^{\prime}+\frac{1}{t_{\mathrm{f}}} \frac{\rho_{\mathrm{p}, 0}}{\rho_{0}}\left(v_{z}^{\prime}-u_{z}^{\prime}\right) .
\end{aligned}
$$

For the particles we get

$$
\begin{aligned}
\frac{\partial \rho_{\mathrm{p}}^{\prime}}{\partial t}+\rho_{\mathrm{p}, 0} \frac{\partial v_{x}^{\prime}}{\partial x}+\rho_{\mathrm{p}, 0} \frac{\partial v_{z}^{\prime}}{\partial z}+v_{z, 0} \frac{\partial \rho_{\mathrm{p}}^{\prime}}{\partial z} & =0, \\
\frac{\partial v_{x}^{\prime}}{\partial t}+v_{z, 0} \frac{\partial v_{x}^{\prime}}{\partial z} & =-\frac{1}{t_{\mathrm{f}}}\left(v_{x}^{\prime}-u_{x}^{\prime}\right), \\
\frac{\partial v_{z}^{\prime}}{\partial t}+v_{z, 0} \frac{\partial v_{z}^{\prime}}{\partial z} & =-\frac{1}{t_{\mathrm{f}}}\left(v_{z}^{\prime}-u_{z}^{\prime}\right) .
\end{aligned}
$$

For modes of the form $A^{\prime} \propto \exp \left(\omega t-i k_{x}-i k_{z}\right)$, we find that the system only has non-zero 
solutions when the determinant is zero,

$$
\begin{aligned}
\left(\omega-i v_{z} k_{z}\right)\left(\omega-i v_{z} k_{z}+t_{\mathrm{f}}^{-1}\right) & \\
& \left(\omega^{2}+\left((1+\varepsilon) t_{\mathrm{f}}^{-1}-i k_{z} v_{z, 0}\right) \omega-\varepsilon t_{\mathrm{f}}^{-1} i k_{z} v_{z, 0}\right)=0 .
\end{aligned}
$$

The first term represents a pure particle mode falling at the terminal velocity, while the second term represents particle motion damped by gas drag. The last factor of this expression has the solutions

$$
\omega=\frac{v_{z} k_{z}}{2} i+\frac{(1+\varepsilon) t_{\mathrm{f}}^{-1}}{2}\left(-1 \pm \sqrt{1-\frac{k_{z}^{2} v_{z, 0}^{2} t_{\mathrm{f}}^{2}}{(1+\varepsilon)^{2}}+2 \frac{(\varepsilon-1) k_{z} v_{z, 0} t_{\mathrm{f}}}{(1+\varepsilon)^{2}}} i\right) .
$$

The real part of the square root term is always below unity $(\operatorname{Re}(\sqrt{1-i x})<1$ for any $x)$, so the perturbation is damped.

In summary, this analysis shows that there are no growing modes under the assumption of incompressibility. Possibly, unstable modes could be found when relaxing the assumption of incompressibility, or by using more realistic equations of state or by exploring nonlocal perturbation techniques. We leave this for future work, given the complexity of such investigations.

\subsection{Toy model dispersion relation}

We start with making the ansatz that

$$
u=\alpha\left(\varepsilon-\varepsilon_{0}\right) v
$$

which removes the explicit dependency on the equations for the gas component. Here $\alpha$ is a proportionality parameter that encapsulates the viscosity dependency and remains to be 


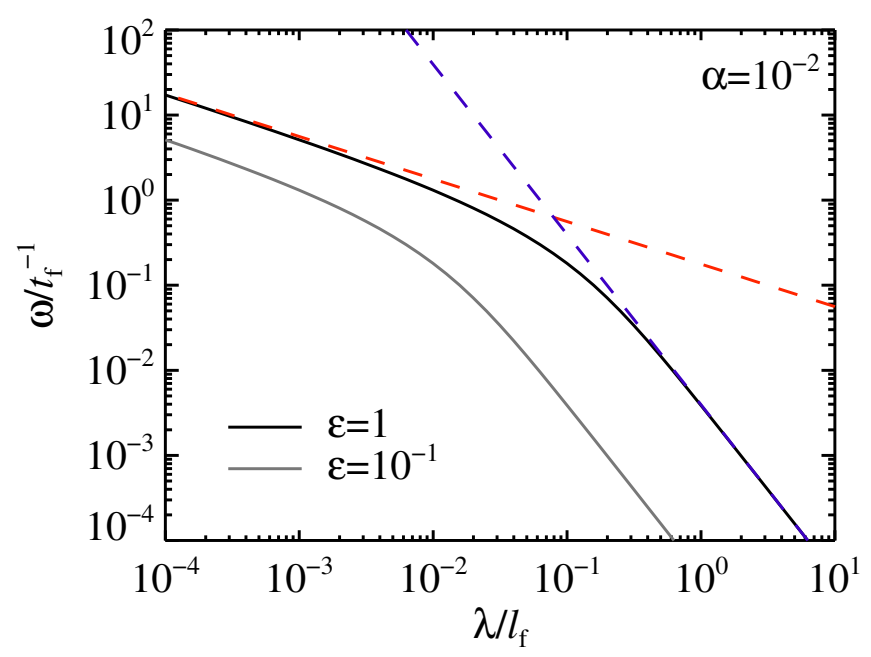

Figure 5.12.: Linear behaviour of the toy model. In black, the real solution to the dispersion relation is given (Eq. 5.10, with $\alpha=0.01$ ). The knee, the largest scale for fast growth, is located at $\lambda_{\text {knee }} \approx 0.08$. The gray curve is the result for a dust-to-gas ratio of 0.1 as opposed to unity. The dashed red line gives the high $k$ approximation (Eq. 5.11) and the dashed blue line gives the low $k$ approximation (Eq. 5.13).

determined through numerical simulations ${ }^{4}$. We also, for simplicity, assume a constant gas density, $\rho=\rho_{0}$. Subsequently, the drag term in the particle momentum equation takes the form

$$
-\frac{1}{t_{\mathrm{f}}}(v-u)=-\frac{v}{t_{\mathrm{f}}}\left[1-\alpha\left(\varepsilon-\varepsilon_{0}\right)\right]
$$

In equilibrium, the dust-to-gas ratio, $\varepsilon=\rho_{\mathrm{p}} / \rho$, is constant. The momentum equation then shows that particles move, as desired, with terminal velocity,

$$
v_{0}=-g t_{\mathrm{f}} .
$$

\footnotetext{
${ }^{4}$ Alternatively, one could assume a more general functional dependency of the form $u(v, \varepsilon)$, similar to Chiang $\&$ Youdin (2010). Then the friction term can be linearised to the form $-\frac{1}{t_{\mathrm{f}}}\left(v+v^{\prime}-u-\frac{\partial u}{\partial v} v^{\prime}-\frac{\partial u}{\partial \varepsilon} \varepsilon^{\prime}\right)$. In order to reduce to expression 5.49, we have to assume $\frac{\partial u}{\partial v}$ is zero around equilibrium. Then, the expression $\alpha v$ corresponds to $\frac{\partial u}{\partial \varepsilon}$.
} 
We now linearize the system of particle equations

$$
\begin{aligned}
\partial_{t} \rho_{\mathrm{p}}^{\prime}+\rho_{\mathrm{p}, 0} \partial_{z} v^{\prime}+v_{0} \partial_{z} \rho_{\mathrm{p}}^{\prime} & =0 \\
\partial_{t} v^{\prime}+v_{0} \partial_{z} v^{\prime} & =-g-\frac{v_{0}+v^{\prime}}{t_{\mathrm{f}}}\left(1-\alpha \varepsilon^{\prime}\right) .
\end{aligned}
$$

The last two terms simplify ${ }^{5}$ to

$$
-g-\frac{v_{0}+v^{\prime}}{t_{\mathrm{f}}}\left(1-\alpha \varepsilon^{\prime}\right)=-\frac{v^{\prime}}{t_{\mathrm{f}}}-g \alpha \frac{\rho_{\mathrm{p}}^{\prime}}{\rho_{0}} .
$$

Taking now modes of the form $A^{\prime} \propto \exp (\omega t-i k z)$ we are left with the following system of equations

$$
\left(\begin{array}{cc}
\omega-i k v_{0} & -i k \rho_{\mathrm{p}, 0} \\
\alpha \frac{g}{\rho_{0}} & \omega-i k v_{0}+\frac{1}{t_{\mathrm{f}}}
\end{array}\right)\left(\begin{array}{c}
\rho_{\mathrm{p}}^{\prime} \\
v^{\prime}
\end{array}\right)=\left(\begin{array}{l}
0 \\
0
\end{array}\right)
$$

Non-zero solutions are found when

$$
\beta^{2}+\frac{\beta}{t_{\mathrm{f}}}+i \alpha \varepsilon_{0} g k=0
$$

where $\beta=\omega-i k v_{0}$. Thus we find

$$
\beta=\frac{1}{2 t_{\mathrm{f}}}\left(-1 \pm \sqrt{1-4 \alpha \varepsilon_{0} g t_{\mathrm{f}}^{2} k i}\right)
$$

where the last term has a positive real part larger than 1 , for any product $\alpha \varepsilon_{0} g t_{\mathrm{f}}^{2}$ different from 0. This reproduces Eq. (5.10), allowing the approximation of the two limit cases, Eq. (5.11) at small scales and Eq. (5.13) at large scales. The shape of the dispersion relation and the

\footnotetext{
${ }^{5}$ When the gas density is not constant the expansion of $\varepsilon=\rho_{\mathrm{p}} / \rho=$ goes as $\varepsilon+\varepsilon^{\prime}=\rho_{\mathrm{p}} / \rho+(1 / \rho) \rho_{\mathrm{p}}^{\prime}-$ $\left(\rho_{\mathrm{p}} / \rho^{2}\right) \rho^{\prime}=\varepsilon+\rho_{\mathrm{p}}^{\prime} / \rho-\varepsilon \rho^{\prime} / \rho$. Then the validity of the model relies on the last term of the expansion to be small.
} 


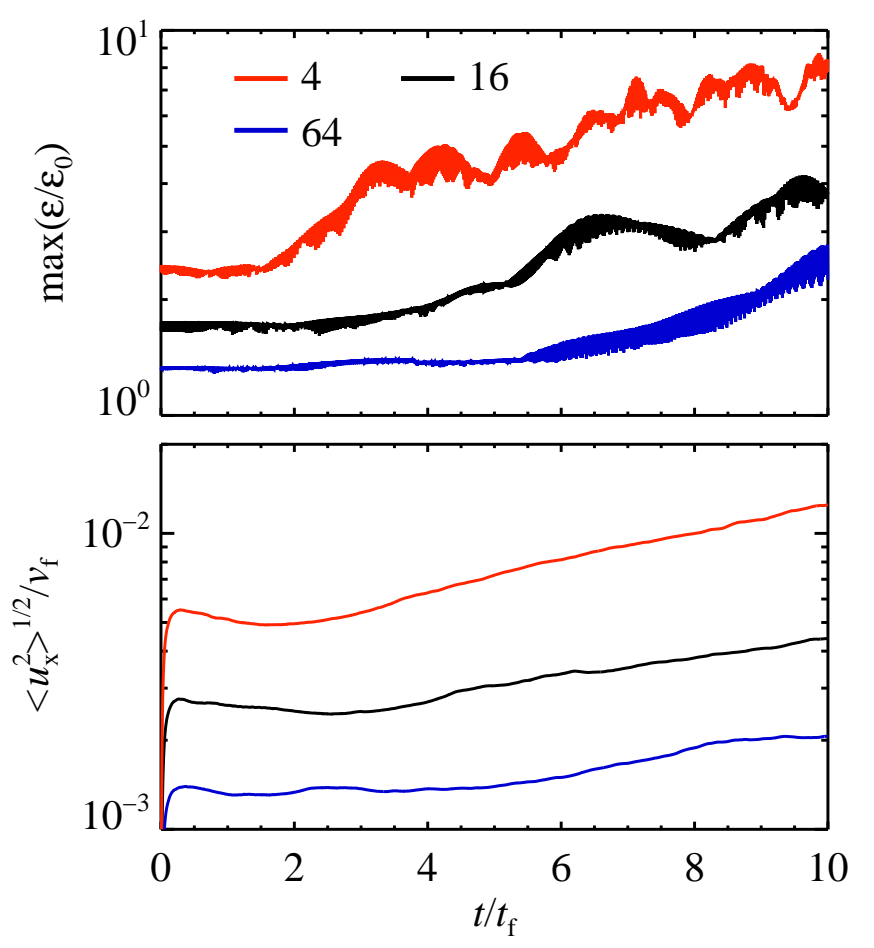

Figure 5.13.: Evolution of the maximal particle density (top) and the horizontal gas dispersion (bottom), for 4, 16, and 32 particles per gridcell (respectively red, black, and blue curves). The initial maximal particle overdensity is reduced from $\varepsilon / \varepsilon_{0}=2.4$ to 1.6 and finally 1.3 , when increasing the particle number by a factor of 4 each time. The lowest particle number simulation shows fastest growth. In higher particle number runs the initial dormant phase persists longer and growth rates become lower. Results from run2.n4, run2, run2.n64.

two limit cases can be inspected in Fig. 5.12.

\subsection{Particle number test}

Particle numbers of 16 superparticles per gridcell are sufficient to capture correctly the evolution of the particle-gas mixture. However, increased particle numbers decrease the noise amplitude that is initially injected. In Fig. 5.13 we show the evolution of the maximal particle density and gas velocity dispersion, as function of the particle number. Because of the non-linear nature of the drafting instability one can see that the decreased noise amplitude 
with increased particle number prolongs a dormant state before the instability comes fully into effect and growth rates decrease moderately. However, if one ignores the protracted dormant phase, growth rates between 16 (our nominal value) and 64 particles per gridcells are undistinguishable, although slower than the 4 particles per gridcell case. 


\section{CHAPTER 6}

\section{Discussion and Perspectives}

By conducting the experiments presented in this thesis, I address a major outstanding problem in the theory of planetesimal formation, which centers on the question of how microscopic dust grains can bind together and result in bodies massive enough to exert gravitational influence on one another. I outlined some of the obstacles to forming planetesimals through direct higherarchical growth due to collisions. While it is clear that the interaction of the particles with the vast gas reservoir must play a role in the evolution of the solid component's density, there is still very active debate about which processes dominate and whether the conditions for them to occur are favourable in the right locations and within observationally determined timescales. In the past decade, SI emerged as potentially playing an important role in overcoming the meter-size barrier.

The interpretation of how the mechanism works remains somewhat illusive. For example, some authors emphasise the importance of rotation, three-dimensional simulations have recently shown that the column metallicity gradient in the vertical direction plays a role.

While my work cannot give definitive answers to these puzzles, it represents the first 
evidence that the symptoms of SI are present in a real physical system. The results of the experiments are most directly comparable to the simulations and semi-analytical calculations presented in Lambrechts et al. (2016) and reproduced in chapter 5, as opposed to the more typical Keplerian sheer-flow model. I emphasise though, that both the simplified sedimentation model and the design of the experimental facility were crafted with the deliberate intent to capture the essential physics of the PPD model that leads to SI. In particular, two-fluid drag-coupled equations described the dynamical evolution of the model, a linear drag law applied, the gas was incompressible, the gas and particle $R e$ low. Some of these assumptions were justified by the large inter-particle separation, or more explicitly, that the momentum diffusion time of the particles was less than the friction time. In chapter 2 I summarised the parameters of the experiments in order to demonstrate that all of these conditions were met in the experimental setup.

Indeed, the system in the laboratory reproduced some of the most celebrated features of the SI, such as the tendency for particle clumps to form, a modification of the velocity statistics, and the exhibition of collective drag-reduction effects. In the analysis of the experiments, it was found that the velocities of the particles were correlated on small scales, especially that their approach velocities became greater in magnitude when their vertical separation was within approximately $4 \mathrm{~mm}$. This result was independent of radial distance, suggesting that the unstable mode is in the z-direction, which is also the direction in which the drag force operates on the particles. This feature is reminiscent of the SI with the unstable mode being in the direction of highest-magnitude particle-gas relative motion for radially drifting particles in a PPD.

In previous numerical studies of the SI, it was noted that the outcome of the instability was the pile-up of dusty regions. By extension, the over-dense regions seem to suffer less drag collectively than they would as isolated particles. In the experiments I found an asymmetry in the particle approach velocity, particles approaching from behind seemed to catch up to 
those that were leading. When considering the dependence of this effect on the local particle number density, the mean velocity decreased for the datasets that were in the continuum drag-law regime, suggesting an effect similar to collective drag reduction. Curiously, the same did not occur for the low pressure data, which is discussed further below.

The quantities I measured are generally in agreement with the theoretical predictions, to the extent that comparison is possible. In chapter 5, figure 5.2, the metrics for the growth of the instability were two-fold: the time evolution of the system maximum density enhancement $\varepsilon / \varepsilon_{0}$, where the subscript 0 indicates the initial background level of mass loading, and the horizontal velocity dispersion $\left\langle u_{x}\right\rangle^{1 / 2} / u_{t}$. Both of these quantities increased with time in the simulations $^{1}$; we noticed exponential growth of the maximum density enhancement which eventually saturated around factors of at least 10 on time sales of less than $8 T_{f}$. Although the horizontal velocity fluctuations were emphasised in the theory, they were shown only because they were a simpler quantity than the vertical velocity fluctuations, which were also present. The experimental results also show local density enhancements by factors of up to several $\times 10$.

In the experiments I did not observe the growth of the particle clumps, but I also did not expect to, since the theoretical prediction was that the instability should have saturated before reaching the measurement volume. Without being able to watch the growth, one might argue that the particle over-dense regions are the result of some initial condition, and not the result of an instability. However, the cross-correlation curves of number density, shown in the appendix figures C.1-C.3, demonstrate that the particle clumps diffused within the their crossing time of the small measurement volume, and so their existence could not be an artefact of the way they are introduced into the flow, because such initial clumps would have long since diffused before the measurement volume is reached. Rather, the clumps

\footnotetext{
${ }^{1}$ I only summarise the results of the $3 \mathrm{D}$ simulations, not the $2 \mathrm{D}$ ones, since the analogue system in the lab is also three dimensional.
} 
form in situ, which is the hallmark of an instability. As a particular note of comparison, in one of the original SI papers, Johansen \& Youdin (2007) calculated the correlation time of particle density and showed that the clumps formed during SI are transient (i.e. they will disperse unless the density amplitude is high enough that a gravitational instability can bind the clump. Certainly we are not hoping for gravitational binding in the experiments, and so we similarly expect the clumps to disperse).

The particle density enhancement and modified settling velocity effects observed in the sedimentation vessel experiments are somewhat more robust than similar effects present in the sedimentation simulations presented in chapter 5 , at least in the sense that the $3 \mathrm{D}$ simulations required $\varepsilon=4$ to arrive at appreciable growth, while in the experimental facility, the results were present for values closer to the canonical $\varepsilon=1$. In chapter 5, it was proposed that the density enhancement of $\sim 10$ reached in the simulations is just sufficient to facilitate chondrule formation, but that reaching the background level of $\varepsilon=4$ would require especially favourable conditions. Since the experiments demonstrate that concentrations of greater than a factor of 10 over the background mass loading are reached already for $\varepsilon \sim 1$, in seems that there would be no problem in facilitating the growth of chondrules, without requiring special pre-concentration mechanisms.

While the initial studies of the SI required a minimum size of the particles in order to be effective (with particle diameters corresponding more to boulders than to pebbles), we don't necessarily find any such requirements in order to reproduce the essential features of the instability either in the sedimentation simulations or experiments; particle clustering apparently occurs if the mass-loading requirement is met. It seems that this lack of size dependence in the current system should have benefits that go in both directions in scale: while the SI could not explain the formation of pebble-sized objects because they are too small, the results in the current study shows that just as one could form chondrules, one should also be able to scale this effect to form planetesimals with the levels of enhancement 
seen in the experiments.

The density enhancement due to the 'traffic jam' effect in the streaming instability simulations can augment the local density from an already concentrated factor of ten, to an additional order of magnitude higher. Although I reported augmentation factors of several tens, this was a conservative estimate based on the median $\mathrm{n}$. If I consider the standard deviation in the mean particle number, I find that the local number density enhancement can be as much as by 180 for $\mathrm{DS}^{2}$. It is therefore possible that the levels of enhancement seen in the experiment are comparable to those seen in the streaming instability, leading to planetesimal formation. I note that this dataset is well into the continuum drag-law regime, but this does not hurt the analogy to the streaming instability, since boulder-sized objects studied in the streaming instability can easily fall above the Stokes-Epstein drag regime transition.

From a mathematical perspective, one should not expect a significantly different outcome between the Stokes or Epstein drag laws, since they are simply linear. However it seems that some differences do arise. For instance, Figure 5.3 shows that sedimenting particle clumps in the simulations, where only an Epstein drag law is assumed, can exceed the mean settling velocity by about one percent. As for the experiments, figure 4.16 shows that the mean settling velocity can be modified by up to 20 percent when the local number density increases, however only for the two data sets where the experiments were conducted at pressures corresponding to $K n<1$. For the lower-pressure case, with $K n \sim 1$, I did not observe the enhanced settling with increased local concentration, even though particle clumping was present. The results of the simulations and experiments are not therefore in conflict, because the simulations considered only the $K n \geq 1$ case and so the weak effect found there is somewhat consistent with the lack of enhanced settling I found for DS2.

\footnotetext{
${ }^{2}$ I acknowledge that this argument is potentially misleading, given that the region of interrogation is of a similar size scale to that of the clump-like features.
} 
The behaviour of the velocity probability distributions was markedly different for the data sets at higher pressure vs. those at lower pressure, with the explanation most likely needing to evoke the roles of viscosity and inter-particle interactions (I do not believe that it can be attributed to turbulence, because the $R e$ was low in all cases). As is already known from sedimentation vessel experiments, viscous interactions of particles with one another via the gas can amplify the velocity dispersion, but as the filling factor increases, the particle accelerations have a damping effect as well. This effect is not considered in the models of the streaming instability, however the work in this thesis takes a step in this direction. When I divided the experimental data into different mass-loading bins, I found that there were more likely to be extreme velocity excursions with respect to the mean dispersion in the low-to-moderate mass loading experiments, but not in the high-mass-loading experiments. At the same time, the velocity dispersion generally decreased with background $\varepsilon$. In the future I would like to better disentangle whether these trends are due to particle-particle scattering by viscous interactions through the gas, which become dissipative at high $\phi$ (especially in cases where the inter-particle separation is low in over-dense regions), or if the trend is strictly reflective of increasing coordinated particle behaviour with higher $\varepsilon$. The role of long-range particle-gas-particle interactions has been extensively studied for flows in the Stokes drag regime, but never before considered for conditions corresponding to $K n \geq 1$. The facility presented here is probably the most appropriate one in existence one to blaze a trail in this unexplored terrain.

The detailed interpretation of the results presented here depends partially upon what has previously been determined to be an important critical control parameter for the SI: the background value of $\varepsilon$. For instance, I conjecture that the relatively noisy figures corresponding to DS4 can be attributed to the fact that most of this DS has $\varepsilon \leq 1$, whereas the other two DS where the effect is more obvious are $\varepsilon \geq 1$. However, the value of $\varepsilon$ depends sensitively on the particle size, which I have only approximately constrained for 
this study. There are complications that arise in the method I used, which depends upon a simplified drag law transition, which in reality should not be so steep, but yet is also not so well understood. Furthermore, pre-determining the particle size distribution before conducting the experiments is not particularly useful, since the experimental apparatus is working in a limit where the terminal velocity of the particles starts to depend upon the pressure: regardless of what the initial distribution of particles is, the flow selects some of the particles and leaves others sitting on the bottom (or else residing in the expansion chamber at the top). In implementing the particle tracking algorithm, I have already extracted gaussian fit parameters from the particle intensity maxima. However, the width of the fit depends partly upon how in-focus the particles are and this needs to be accounted for. An alternative method for determining particle size is to measure the intensity variations in the shadow images. However, this has to be done carefully to account for illumination inhomogeneities in the images. While resolving these matters will further constrain the particle size and clarify the exact mass-loading dependencies, I note that the primary findings, that particle clumping via aerodynamic focusing occurs under several conditions close to the Stokes-Epstein transition for $\varepsilon$ order unity, will not change.

In the experiments using LPT, I found that the particle velocities correlated as a function of scale, and that the longest length-scale of the correlation may have been larger than the measurement volume. In the future, one might revisit the experiments using an observation method that covers larger regions to understand how the scale dependence works.

The results presented here may also bridge between the particle concentration and gravitational instability phases of the planetesimal formation process. Nesvorný et al. (2010) modelled the gravitational collapse of a cloud of pebbles, assuming that they have been concentrated due to SI. Follow-up studies (Wahlberg Jansson \& Johansen 2014, Wahlberg Jansson et al. 2017) include experimentally-derived collisional outcomes, taken from Güttler et al. (2010) and Bukhari Syed et al. (2017). Such studies yield predictions on the porosity of 
planetesimals which are now being tested with data arriving from missions such as Rosetta and New Horizons, which directly probe the surface-layer features of comet 67P/ChuryumovGerasimenko and Pluto (Blum et al. 2014). Thus far, the collapsing pebble-cloud models begin with a cloud where the relative particle velocities are due to their free fall velocities, although it may be that the particles in the clump are swarming internally as well. In the particle-settling experiments described herein, one of the measurable statistics is the particle relative-velocity dispersion within the unstable, high-concentration regions. Perhaps a better understanding the underlying form of these velocity distributions and how they vary for different conditions could eventually supply a model enhancement for the study of collapsing pebble clouds. 


\section{A. Estimated parameters relevant to particle-turbulence interactions in PPDs}

The gas properties in the PPDs depend on the gas density $\rho$, which can vary over many orders of magnitudes. When estimating the parameters, we assume that the gas behaves like an ideal gas at a temperature of approximately $300 \mathrm{~K}$. The dynamic viscosity $\mu$ of an ideal gas is independent of the gas density. It depends only on the temperature and the molecular constitution. At $300 \mathrm{~K}$, the dynamic viscosity of most gas is $\mu \approx 10^{-5} \mathrm{~kg} /(\mathrm{m} \cdot \mathrm{s})$. The kinematic viscosity is simply $v=\mu / \rho$.

The mean free path of an ideal gas is related to the kinematic viscosity by $\lambda=2 v / a$, where $a$ is the mean molecular velocity and is between $10^{2}-10^{3} \mathrm{~m} / \mathrm{s}$ for most gas at $300 \mathrm{~K}$. We will use $a \approx 10^{2} \mathrm{~m} / \mathrm{s}$ when estimating the mean free path $\lambda$.

To estimate turbulence parameters, we need to estimate the energy dissipation rate $\varepsilon$. We use the following very crude estimate: The turbulent fluctuation velocity is $u^{\prime} \approx 50 \mathrm{~m} / \mathrm{s}$, which is taken as the same as the mean slipping velocity between the keplerian velocity of the particles and the sub-keplerian velocity of the gas (Youdin 2010). Note that this velocity is 
already large compared to the speed of sound of the gas at that temperature (about the same as a) and we might have to consider compressibility effects. The energy injection scale is taken as the height of the PPDs, which usually is estimated as $h=0.1 r$. At $r=1 \mathrm{AU}$, this gives $h=10^{10} \mathrm{~m}$. Therefore, the energy dissipation rate is $\varepsilon \approx u^{\prime 3} / L \approx u^{\prime 3} / h \approx 50^{3} / 10^{10} \approx 10^{-5}$ $\mathrm{m}^{2} / \mathrm{s}^{3}$. Then the rest of the turbulence parameters are estimated according to the standard definition, e.g. the Kolmogorov length scale is $\eta=\left(v^{3} / \varepsilon\right)^{1 / 4}$, the Kolmogorv time scale is $\tau_{\eta}=(v / \varepsilon)^{1 / 2}$, and the Kolmogorov velocity is $u_{\eta}=(v \varepsilon)^{1 / 4}$. The weak dependence of these quantities on $\varepsilon$ suggests that even if we make a large error in $\varepsilon$, these quantities are not heavily affected, e.g., a 4 orders of magnitudes of change in $\varepsilon$ only changes $\eta$ and $u_{\eta}$ by a factor of 10 . The Reynolds number can be estimated as $R_{\lambda} \approx(L / \eta)^{2 / 3}$.

With the gas and turbulence parameters, we can further estimate the dynamic properties of solid particles with different sizes. To simplify the estimation, we assume the particle is spheric and its material density is $\rho_{p}=3 \times 10^{3} \mathrm{~kg} / \mathrm{m}^{3}$, which is a usual assumption in astrophysics journal articles. The drag force on a solid sphere moving at constant velocity $u_{p}$ in a still gas is a complicated problem by itself. We take a much simplified approximation: If particle diameter $d_{p}$ is small compared to the mean free path $\lambda$ of the gas molecules, the particle experiences a drag that is due to the collision with the free molecules. If the particle velocity is small compared to the molecule velocity, i.e., $u_{p} \ll a$, the drag is called the Epstein drag (Epstein 1924, Armitage 2010):

$$
f_{D}=\frac{\pi}{3} d_{p}^{2} \rho a u_{p}=\frac{2 \pi}{3} \frac{d_{p}}{\lambda} \rho v u_{p} d_{p}
$$

For large particles $d_{p} \gg \lambda$, the gas can be treated as a continuum and the drag depends on the Reynolds number $R e_{p}=d_{p} u_{p} / v$. If $R e_{p} \ll 1$ the drag force is given by the Stokes formula:

$$
f_{D}=3 \pi \rho v u_{p} d_{p}
$$


Comparing the Epstein drag and Stokes drag, one finds that the two cross over at $d_{p}=(9 / 2) \lambda$. In our estimation, we ignore the transition regime and use the Epstein drag for $d_{p} / \lambda \leq 9 / 2$ and the Stokes drag for larger particles. If $R e_{p} \gg 1$, the flow around the particle is turbulent and the drag force can be written as

$$
f_{D}=C_{d} \frac{1}{2} \rho \frac{\pi}{4} d_{p}^{2} u_{p}^{2}=\frac{\pi}{8} C_{d} R e_{p} \rho v u_{p} d_{p}
$$

where $C_{d}$ is an empirical coefficient. For large $R e_{p}$, it has an approximate value of $C_{d} \approx$ $0.4-0.5$. The crossover from Stokes drag to turbulence drag formula occurs at $R e_{p}=24 / C_{d}$. We again neglect the transition regime and use the Stokes drag for $R e_{p} \leq 24 / C_{d}$ and the turbulence drag for the rest. We note here that both the Epstein drag and the turbulence drag are proportional to $d_{p}^{2}$, while the Stokes drag is linear in $d_{p}$. Having known the drag force on a particle, the response time of a particle, $t_{p}$, can be defined as the time required to fully stop a particle whose initial velocity is $u_{p}$ and is subject to the corresponding constant drag force, i.e.

$$
t_{p} \equiv \frac{m_{p} u_{p}}{f_{D}}=\frac{(\pi / 6) d_{p}^{3} \rho_{p} u_{p}}{f_{D}}
$$

In different regimes, this particle response time can be written explicitly as for $d_{p} \leq(9 / 2) \lambda$,

$$
t_{p}=\frac{1}{2} \frac{\rho_{p}}{\rho} \frac{d_{p}}{a}=\frac{1}{4} \frac{\rho_{p} d_{p}^{2}}{\mu} \frac{\lambda}{d_{p}}
$$

and for $d_{p} \geq(9 / 2) \lambda$ and $R e_{p} \leq 24 / C_{d}$,

$$
t_{p}=\frac{1}{18} \frac{\rho_{p} d_{p}^{2}}{\mu}
$$

and for $\operatorname{Re}_{p} \geq 24 / C_{d}$,

$$
t_{p}=\frac{4}{3 C_{d}} \frac{\rho_{p}}{\rho} \frac{d_{p}}{u_{p}}=\frac{4}{3 C_{d}} \frac{\rho_{p} d_{p}^{2}}{\mu} R e_{p}^{-1}
$$


The response time defined in this way is the same as the usual viscous relaxation time defined for Stokes flows. Note that because of the dependence of $f_{D}$ on $d_{p}$, the response times in both the Epstein regime and the turbulence regime depends linearly on $d_{p}$, but in the Stokes flow regime it depends on $d_{p}^{2}$. A parameter to measure particle inertial in a turbulent field is the ratio of the particle response time to the Kolmogorov time, i.e., the Stokes number $S t=t_{p} / \tau_{\eta}$

Table 6.1 summarizes these parameters for different gas densities. In this table, particles with 3 different sizes: $d_{p}=10^{-2}, 10^{-1}$, and $1 \mathrm{~m}$ are listed. It can be seen that for all these particles, the Stokes number is much larger than unity and hence they can be considered as massive. Therefore, for these boulders, the turbulence field does not seem to affect their motion considerably. If there is no other source of disturbance, the relative velocities between these particles can be very small! Probably the collision is mainly due to the difference in radial drift, similar to the gravitational settling of cloud droplets? 
A. Estimated parameters relevant to particle-turbulence interactions in PPDs 


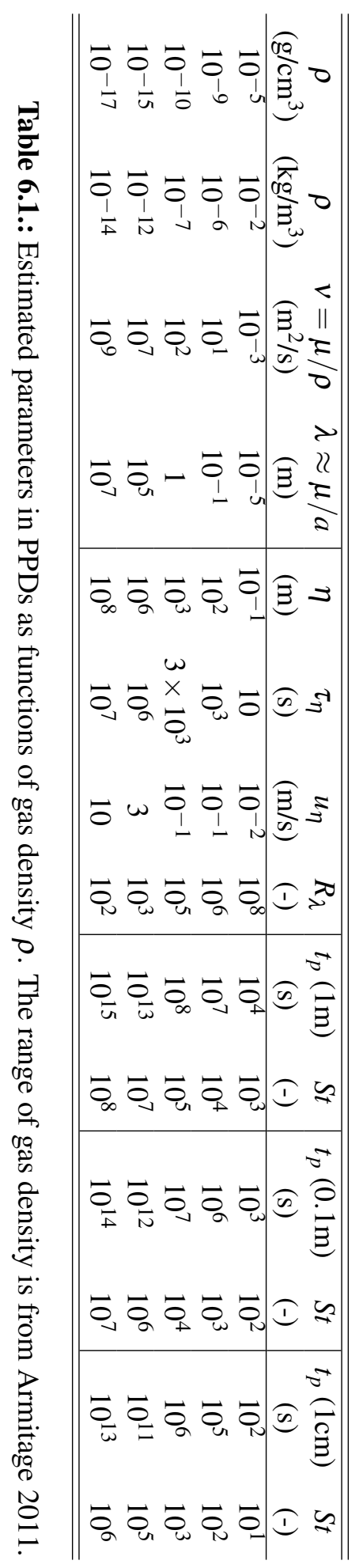


B. Global Variation in Number Density 

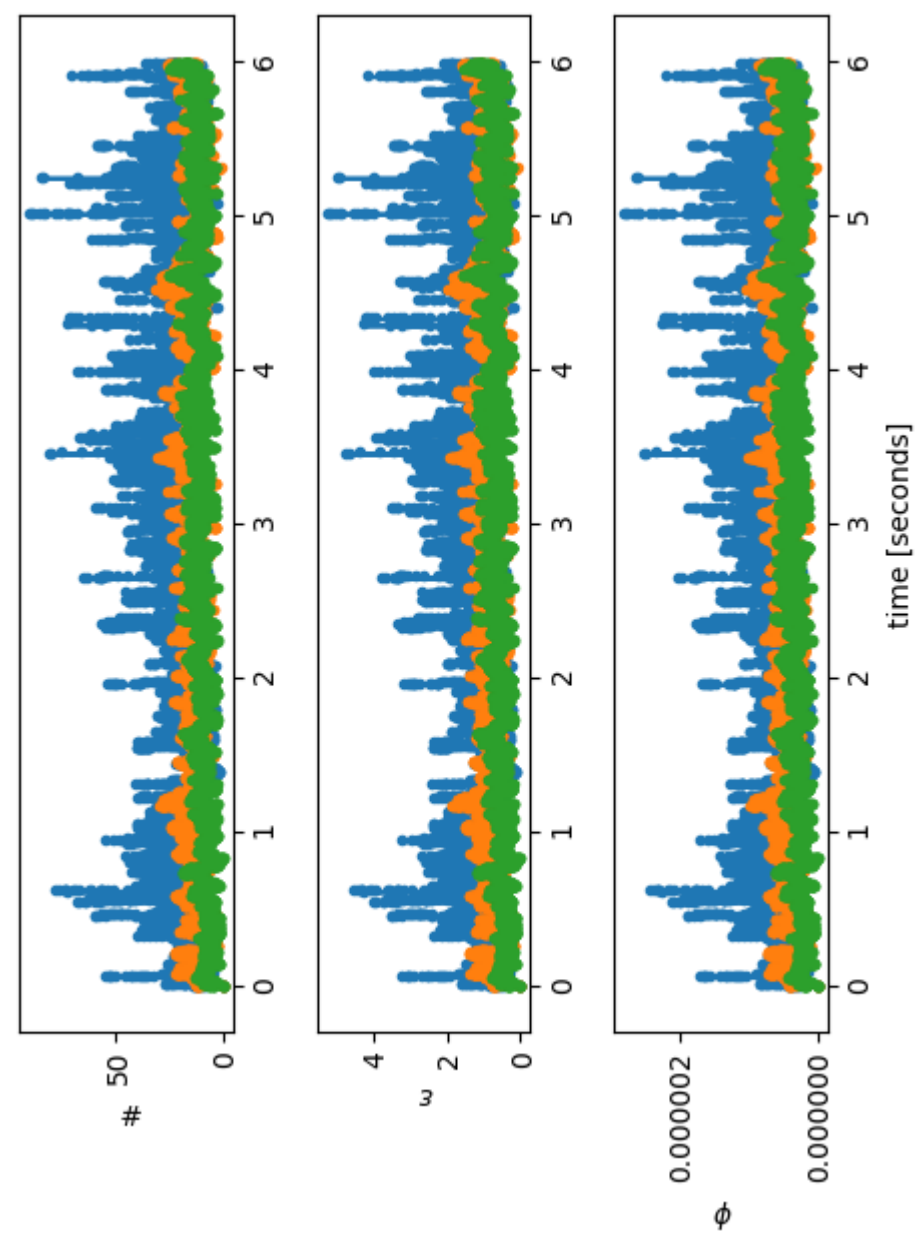

Figure B.1.: Variation in number density, mass loading and filling factor for several experiments in DS2, including the lowest and highest number densities, as well as the median number density. 

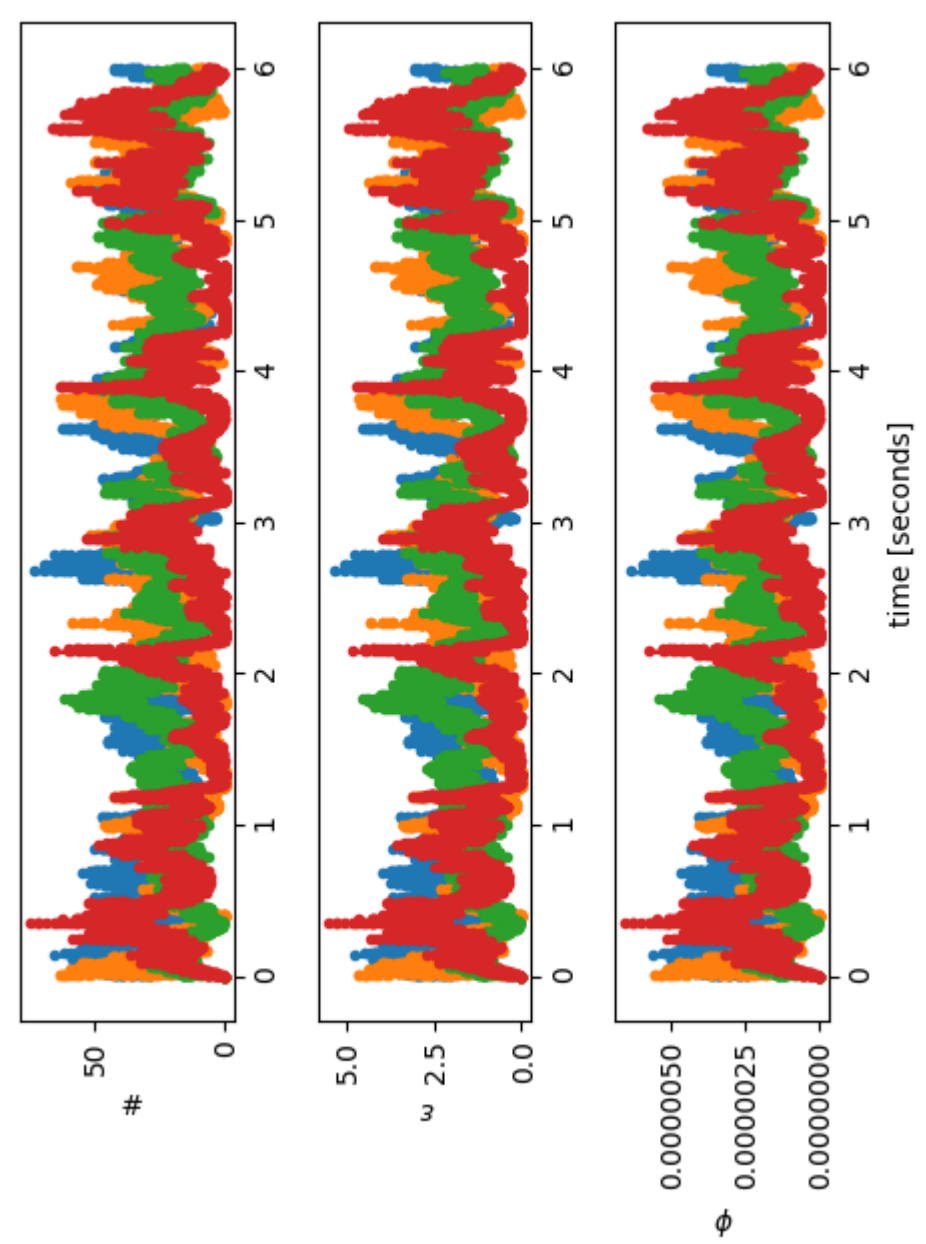

Figure B.2.: Variation in number density, mass loading and filling factor for several experiments in DS3, including the lowest and highest number densities, as well as the median number density. 

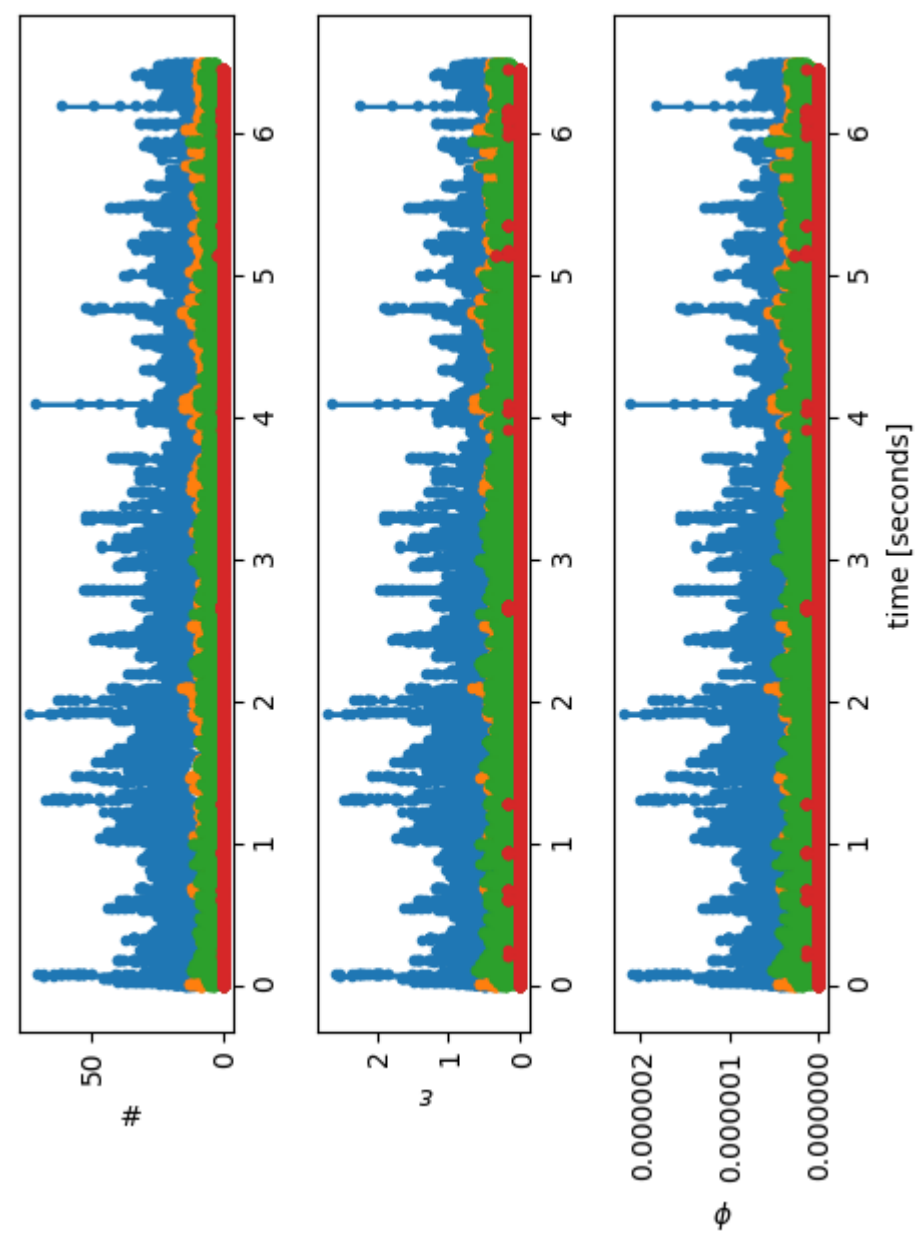

Figure B.3.: Variation in number density, mass loading and filling factor for $\mathrm{r}$ several experiments in DS4, including the lowest and highest number densities, as well as the median number density. 
C. Local Variation in Number Density 


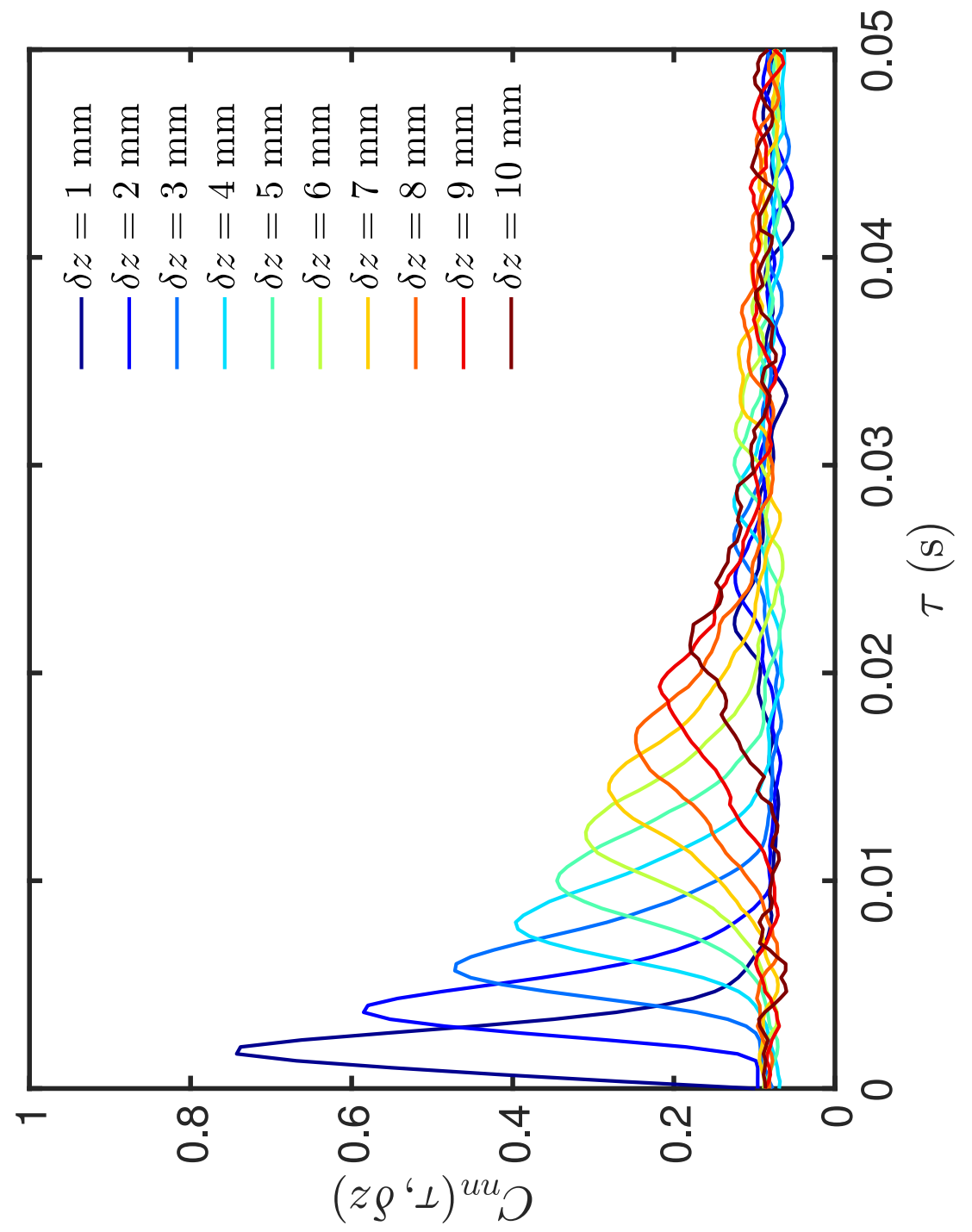

Figure C.1.: Cross correlation of number density for DS2 as a function of time and conditioned upon height in the measuremet volume. 


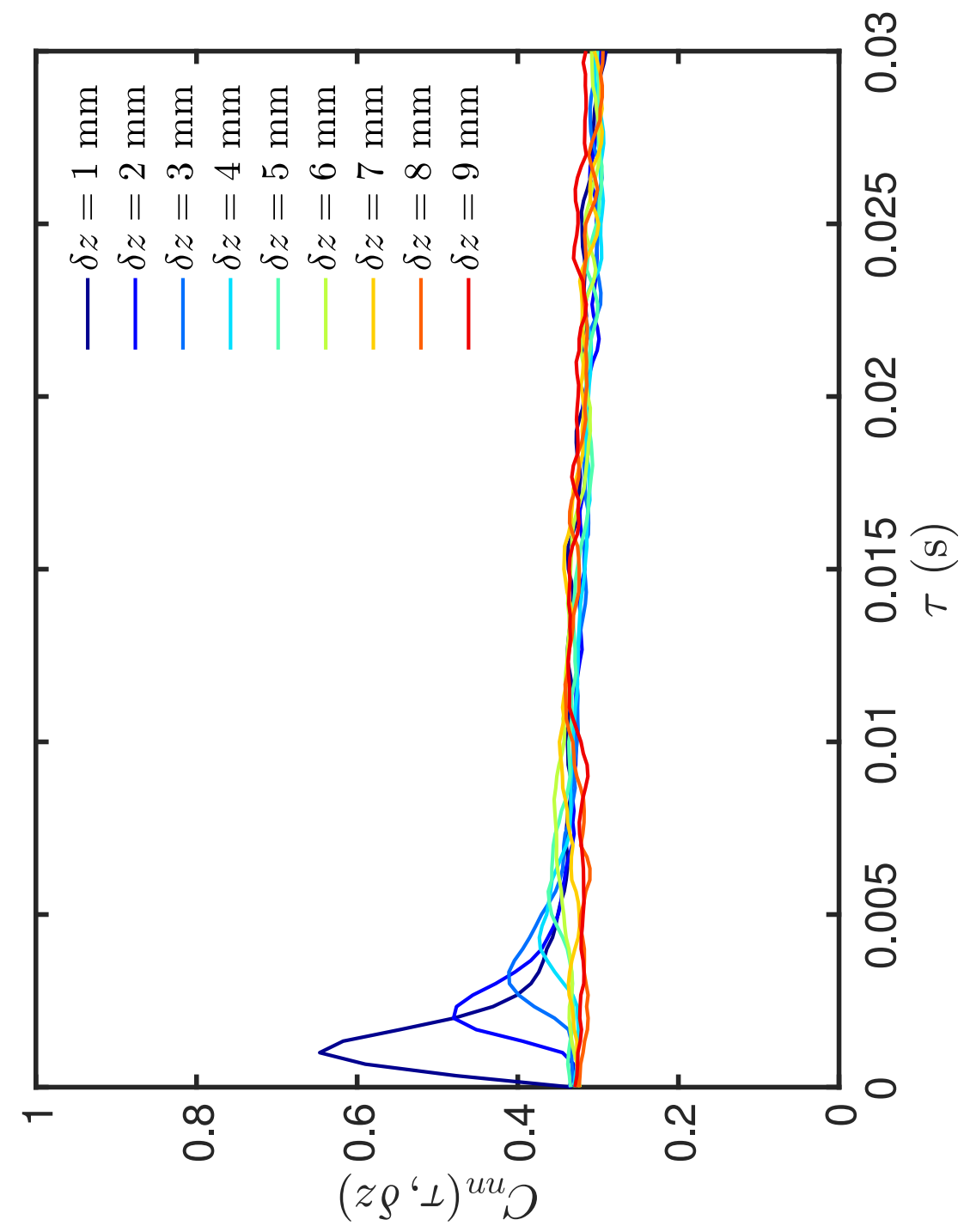

Figure C.2.: Cross correlation of number density for DS3 as a function of time and conditioned upon height in the measuremet volume. 


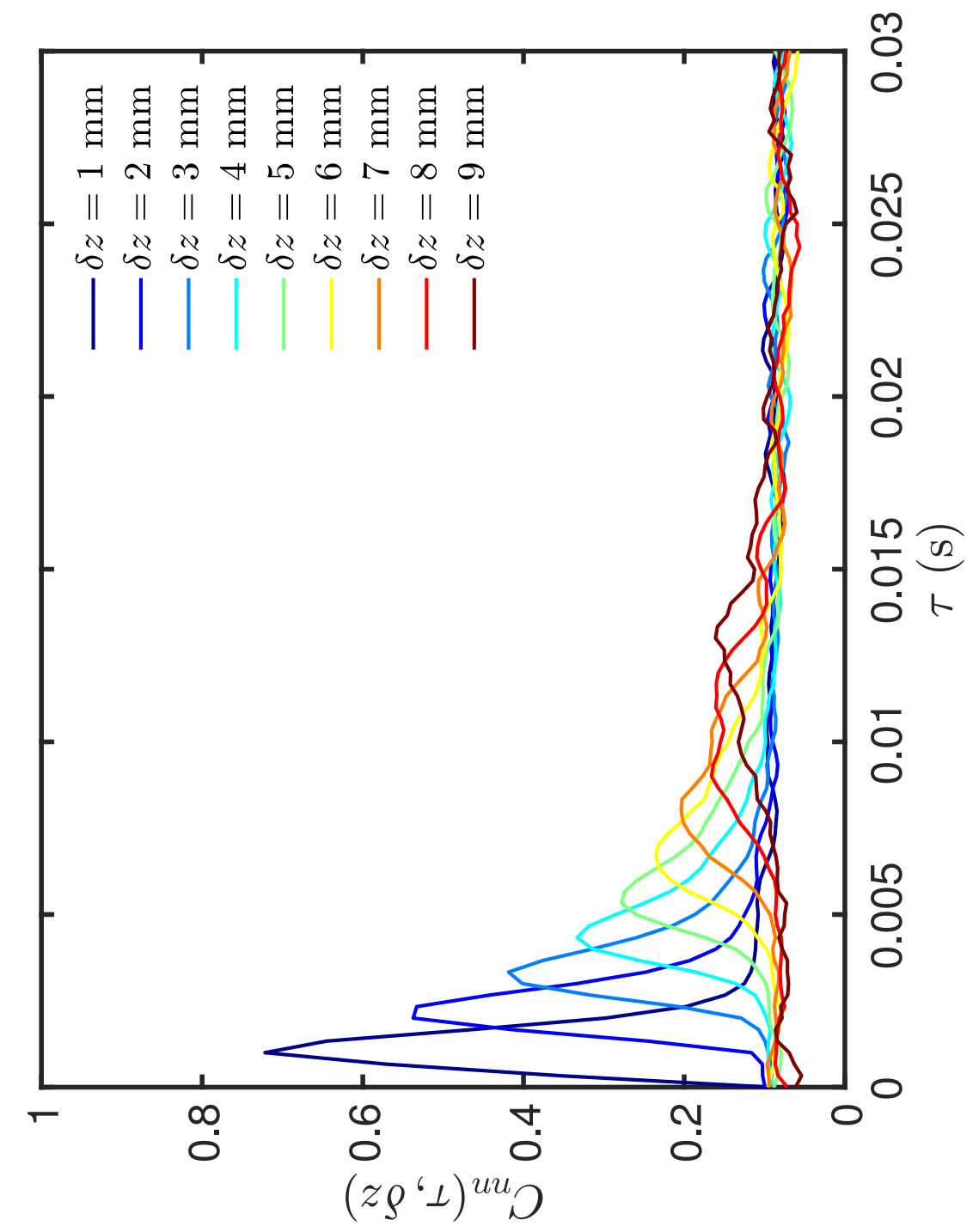

Figure C.3.: Cross correlation of number density for DS4 as a function of time and conditioned upon height in the measuremet volume. 


\section{Bibliography}

Alexander, C. M. O. ., Grossman, J. N., Ebel, D. S. \& Ciesla, F. J. 2008 The Formation Conditions of Chondrules and Chondrites. Science 320, 1617.

ARmitage, P. J. 2010 Astrophysics of Planet Formation. Cambridge, University Press.

Armitage, P. J. 2011 Dynamics of Protoplanetary Disks. Annual Review of Astronomy and Astrophysics 49 (1), 195-236.

BAI, X.-N. \& Stone, J. M. $2010 a$ Dynamics of Solids in the Midplane of Protoplanetary Disks: Implications for Planetesimal Formation. The Astrophysical Journal 722, 14371459.

Bai, X.-N. \& Stone, J. M. $2010 b$ Particle-gas Dynamics with Athena: Method and Convergence. The Astrophysical Journal Supplement Series 190, 297-310.

Bai, X.-N. \& Stone, J. M. 2010c The Effect of the Radial Pressure Gradient in Protoplanetary Disks on Planetesimal Formation. The Astrophysical Journal Letters 722, L220-L223.

Balachandar, S. \& Eaton, J. K. 2010 Turbulent dispersed multiphase flow. Annual Review of Fluid Mechanics 42, 111-133. 
Balbus, S. A. 2009 Magnetohydrodynamics of Protostellar Disks. arXiv.org astro-ph.SR.

BAlbus, S. A. \& HaWley, J. F. 1991 A powerful local shear instability in weakly magnetized disks. I - Linear analysis. II - Nonlinear evolution. The Astrophysical Journal 376, 214-233.

BARge, P. \& Sommeria, J. 1995 Did planet formation begin inside persistent gaseous vortices? Astronomy \& Astrophysics 295, L1-L4.

BATCHELOR, G. K. 1972 Sedimentation in a dilute dispersion of spheres. Journal of Fluid Mechanics 52, 245-268.

BATCHELOR, G. K. 1972 Sedimentation in a dilute dispersion of spheres. Journal of Fluid Mechanics 52, 245-268.

Bate, M. R. \& LorÉn-Aguilar, P. 2017 On the dynamics of dust during protostellar collapse. Monthly Notices of the Royal Astronomical Society 465, 1089-1094.

Bell, C. P. M., Naylor, T., Mayne, N. J., Jeffries, R. D. \& Littlefair, S. P. 2013 Pre-main-sequence isochrones - II. Revising star and planet formation time-scales. Monthly Notices of the Royal Astronomical Society 434, 806-831.

Birnstiel, T., Klahr, H. \& Ercolano, B. 2012 A simple model for the evolution of the dust population in protoplanetary disks. Astronomy \& Astrophysics 539, A148.

Bitsch, B., Johansen, A., Lambrechts, M. \& Morbidelli, A. 2015 The structure of protoplanetary discs around evolving young stars. Astronomy \& Astrophysics 575, A28.

Blum, J., Beitz, E., Bukhari, M., Gundlach, B., Hagemann, J.-H., Heisselmann, D., Kothe, S., Schräpler, R., von Borstel, I. \& Weidling, R. 2014 Laboratory Drop Towers for the Experimental Simulation of Dust-aggregate Collisions in 
the Early Solar System. JOVE, Journal of Visualized Experiments, Vol. 88, id. e51541, 8 pp. 88, e51541.

Blum, J., Gundlach, B., Mühle, S. \& Trigo-Rodriguez, J. M. 2014 Comets formed in solar-nebula instabilities!-An experimental and modeling attempt to relate the activity of comets to their formation process. Icarus $\mathbf{2 3 5}, 156-169$.

Blum, J., Schräpler, R., Davidsson, B. J. R. \& Trigo-Rodríguez, J. M. 2006 The Physics of Protoplanetesimal Dust Agglomerates. I. Mechanical Properties and Relations to Primitive Bodies in the Solar System. The Astrophysical Journal 652, 1768-1781.

BLUM, J. \& WURM, G. 2000 Experiments on Sticking, Restructuring, and Fragmentation of Preplanetary Dust Aggregates. Icarus 143, 138-146.

BLUM, J. \& WURM, G. 2008 The growth mechanisms of macroscopic bodies in protoplanetary disks. Annu Rev Astron Astrophys .

Blum, J., Wurm, G., Kempf, S. \& Henning, T. 1996 The Brownian Motion of Dust Particles in the Solar Nebula: an Experimental Approach to the Problem of Pre-planetary Dust Aggregation. Icarus 124, 441-451.

Blum, J., Wurm, G., Kempf, S., Poppe, T., Klahr, H., Kozasa, T., Rott, M., Henning, T., Dorschner, J., Schräpler, R., Keller, H. U., Markiewicz, W. J., Mann, I., Gustafson, B. A., Giovane, F., Neuhaus, D., Fechtig, H., Grün, E., Feuerbacher, B., Kochan, H., Ratke, L., El Goresy, A., Morfill, G., Weidenschilling, S. J., Schwehm, G., Metzler, K. \& IP, W.-H. 2000 Growth and Form of Planetary Seedlings: Results from a Microgravity Aggregation Experiment. Physical Review Letters 85, 2426-2429. 
Blum, J., Wurm, G., Poppe, T. \& Heim, L.-O. 1998 Aspects of Laboratory Dust Aggregation with Relevance to the Formation of Planetesimals. Earth Moon and Planets 80, 285-309.

Blum, J., Wurm, G., Poppe, T., Kempf, S., Fiethe, B., Giel, M., Offterdinger, P., Neuhaus, D., Rott, M., Giovane, F. \& Gustafson, B. 1999 The cosmic dust aggregation experiment CODAG. Measurement Science and Technology 10, 836-844.

Bobeck, P. 2006 Henry Darcy in his own words. Hydrogeology Journal 14, 998-1004.

Boss, A. P. 1996 A concise guide to chondrule formation. In Chondrules and the Protoplanetary Disk (ed. R. Hewins, R. Jones \& E. Scott), pp. 257-263.

Bourgoin, M. 2006 The Role of Pair Dispersion in Turbulent Flow. Science 311 (5762), $835-838$.

BRANDENBURG, A. 2003 Computational aspects of astrophysical MHD and turbulence, p. 269. London: Taylor and Francis Group.

BRANDEnburg, A. \& Dobler, W. $2002 a$ Hydromagnetic turbulence in computer simulations. Computer Physics Communications 147, 471-475.

BRANDENBURG, A. \& Dobler, W. $2002 b$ Hydromagnetic turbulence in computer simulations. Computer Physics Communications 147, 471-475.

Brauer, F., Dullemond, C. P. \& Henning, T. 2008a Coagulation, fragmentation and radial motion of solid particles in protoplanetary disks. Astronomy \& Astrophysics $\mathbf{4 8 0}$, 859-877.

Brauer, F., Dullemond, C. P. \& Henning, T. 2008b Coagulation, fragmentation and radial motion of solid particles in protoplanetary disks. Astronomy \& Astrophysics $\mathbf{4 8 0}$, 859-877. 
Brauer, F., Henning, T. \& Dullemond, C. P. 2008c Planetesimal formation near the snow line in MRI-driven turbulent protoplanetary disks. Astronomy \& Astrophysics $\mathbf{4 8 7}$, L1-L4.

Bukhari Syed, M., Blum, J., Wahlberg Jansson, K. \& Johansen, A. 2017 The Role of Pebble Fragmentation in Planetesimal Formation. I. Experimental Study. The Astrophysical Journal 834, 145.

Carrera, D., Johansen, A. \& Davies, M. B. 2015 How to form planetesimals from mm-sized chondrules and chondrule aggregates. Astronomy \& Astrophysics 579, A43.

Chiang, E. \& Youdin, A. N. 2010 Forming Planetesimals in Solar and Extrasolar Nebulae. Annual Review of Earth and Planetary Sciences 38, 493-522.

COPERNICUS, N. 1543 D revolutionibus orbium coelestium.

Cross, M. \& Greenside, H. 2009 Pattern Formation and Dynamics in Nonequilibrium Systems. Cambridge University Press, cambridge Books Online.

Cuzzi, J. N. \& AleXAnder, C. M. O. 2006 Chondrule formation in particle-rich nebular regions at least hundreds of kilometres across. Nature 441, 483-485.

Cuzzi, J. N., Dobrovolskis, A. R. \& Champney, J. M. 1993 Particle-gas dynamics in the midplane of a protoplanetary nebula. Icarus 106 (1), 102-134.

Cuzzi, J. N., Hogan, R. C., Paque, J. M. \& Dobrovolskis, A. R. $2001 a$ Sizeselective Concentration of Chondrules and Other Small Particles in Protoplanetary Nebula Turbulence. The Astrophysical Journal 546, 496-508.

Cuzzi, J. N., Hogan, R. C., Paque, J. M. \& Dobrovolskis, A. R. $2001 b$ Sizeselective Concentration of Chondrules and Other Small Particles in Protoplanetary Nebula Turbulence. The Astrophysical Journal 546, 496-508. 
Cuzzi, J. N. \& Weidenschilling, S. J. 2006 Particle-Gas Dynamics and Primary Accretion, pp. 353-381. Meteorites and the Early Solar System II.

Dominik, C., Blum, J., Cuzzi, J. N. \& Wurm, G. 2007 Growth of Dust as the Initial Step Toward Planet Formation. In Protostars and Planets V (ed. B. Reipurth, D. Jewitt \& K. Keil), pp. 783-800.

Dominik, C. \& Tielens, A. G. G. M. 1997 The Physics of Dust Coagulation and the Structure of Dust Aggregates in Space. The Astrophysical Journal 480, 647.

DRAZIN, P. G. \& ReID, W. H. 2004 Hydrodynamic Stability, 2nd edn. Cambridge University Press, cambridge Books Online.

Drazin, P. G. \& ReID, W. H. 2004 Hydrodynamic Stability. Cambridge, University Press.

DrA̧ŻKowsKA, J. \& DUllemond, C. P. 2014 Can dust coagulation trigger streaming instability? Astronomy \& Astrophysics 572, A78.

Dubrulle, B., Dauchot, O., Daviaud, F., Longaretti, P. Y., Richard, D. \& ZAHN, J. P. 2005 Stability and turbulent transport in Taylor-Couette flow from analysis of experimental data. Physics of Fluids 17 (9), 095103-095103-19.

Dubrulle, B., Morfill, G. \& Sterzik, M. 1995 The dust subdisk in the protoplanetary nebula. Icarus 114 (2), 237 - 246.

Dullemond, C. P., Hollenbach, D., Kamp, I. \& D’ Alessio, P. 2007 Models of the Structure and Evolution of Protoplanetary Disks. In Protostars and Planets V (ed. B. Reipurth, D. Jewitt \& K. Keil), pp. 555-572.

EINSTEIN, A. 1905 Über die von der molekularkinetischen Theorie der Wärme geforderte Bewegung von in ruhenden Flüssigkeiten suspendierten Teilchen. Annalen der Physik 322, $549-560$. 
Epstein, P. S. 1924 On the Resistance Experienced by Spheres in their Motion through Gases. Physical Review 23, 710-733.

FENG, J., HU, H. H. \& JOSEPH, D. D. 1994 Direct simulation of initial value problems for the motion of solid bodies in a Newtonian fluid Part 1. Sedimentation. Journal of Fluid Mechanics 261, 95-134.

Fischer, D. A. \& VAlenti, J. 2005 The Planet-Metallicity Correlation. The Astrophysical Journal 622, 1102-1117.

FLOWER, D. 2016 The eslab 50 symposium - spacecraft at comets from 1p/halley to 67p/churyumov-gerasimenko. Monthly Notices of the Royal Astronomical Society, Special Issue 462.

Fortes, A. F., Joseph, D. D. \& LundGRen, T. S. 1987 Nonlinear mechanics of fluidization of beds of spherical particles. Journal of Fluid Mechanics 177, 467-483.

GALILEI, G. 1655 Sidereus nuncius magna, longeque admirabilia spectacula pandens ... quae a Galileo Galileo.

Gibert, M., Xu, H. \& Bodenschatz, E. 2010 Inertial effects on two-particle relative dispersion in turbulent flows. EPL (Europhysics Letters) 90 (6), 64005.

Goldreich, P. \& WARD, W. R. 1973 The Formation of Planetesimals. The Astrophysical Journal 183, 1051-1062.

Goodman, J. \& Pindor, B. 2000 Secular Instability and Planetesimal Formation in the Dust Layer. Icarus 148, 537-549.

GuAzZELli, É. \& Hinchdoychev, J. 2011 Fluctuations and Instability in Sedimentation. Annual Review of Fluid Mechanics 43, 97-116. 
Guazzelli, É., Morris, J. \& Pic, S. 2011 A Physical Introduction to Suspension Dynamics. Cambridge University Press.

GuAZZELli, Ã. \& HINCH, J. 2011 Fluctuations and instability in sedimentation. Annual Review of Fluid Mechanics 43 (1), 97-116.

Guillot, T., IDA, S. \& ORMEL, C. W. 2014 On the filtering and processing of dust by planetesimals. I. Derivation of collision probabilities for non-drifting planetesimals. Astronomy \& Astrophysics 572, A72.

Güttler, C., Blum, J., Zsom, A., Ormel, C. W. \& Dullemond, C. P. 2010 The outcome of protoplanetary dust growth: pebbles, boulders, or planetesimals?. I. Mapping the zoo of laboratory collision experiments. Astronomy \& Astrophysics 513, A56.

HAISCH, JR., K. E., LADA, E. A. \& LADA, C. J. 2001 Disk Frequencies and Lifetimes in Young Clusters. Astrophysical Journal Letters 553, L153-L156.

HAUGEN, N. E. L. \& BRANDENBURG, A. 2004 Inertial range scaling in numerical turbulence with hyperviscosity. Physical Review E 70 (2), 026405.

HAYASHI, C. 1981 Structure of the Solar Nebula, Growth and Decay of Magnetic Fields and Effects of Magnetic and Turbulent Viscosities on the Nebula. Progress of Theoretical Physics Supplement 70, 35-53.

HAYAShi, C., NAKAZAWA, K. \& NAKAGAWA, Y. 1985 Formation of the solar system. In Protostars and Planets II (ed. D. C. Black \& M. S. Matthews), pp. 1100-1153.

Heim, L.-O., Blum, J., Preuss, M. \& Butt, H.-J. 1999 Adhesion and Friction Forces between Spherical Micrometer-Sized Particles. Physical Review Letters 83, 3328-3331.

HendriX, T. \& KePPEns, R. 2014 Effect of dust on Kelvin-Helmholtz instabilities. Astronomy \& Astrophysics 562, A114. 
Hersant, F., Dubrulle, B. \& Hure, J. M. 2005 Turbulence in circumstellar disks. Astronomy and Astrophysics 429, 531-542.

Hughes, A. M., Wilner, D. J., Andrews, S. M., Qi, C. \& Hogerheijde, M. R. 2011 Empirical constraints on turbulence in protoplanetary accretion disks. The Astrophysical Journal 727 (2), 85.

IKOMA, M., NAKAZAWA, K. \& EMORI, H. 2000 Formation of Giant Planets: Dependences on Core Accretion Rate and Grain Opacity. The Astrophysical Journal 537, 1013-1025.

JACQUET, E., BAlBus, S. \& LATTER, H. 2011 On linear dust-gas streaming instabilities in protoplanetary discs. Monthly Notices of the Royal Astronomical Society 415, 3591-3598.

JACQuet, E. \& Thompson, C. 2014 Chondrule Destruction in Nebular Shocks. The Astrophysical Journal 797, 30.

Johansen, A., Blum, J., Tanaka, H., Ormel, C., Bizzarro, M. \& Rickman, H. 2014 The Multifaceted Planetesimal Formation Process. Protostars and Planets VI pp. $547-570$.

Johansen, A., Henning, T. \& Klahr, H. 2006 Dust Sedimentation and Self-sustained Kelvin-Helmholtz Turbulence in Protoplanetary Disk Midplanes. The Astrophysical journal 643, 1219-1232.

Johansen, A. \& Klahr, H. 2005 Dust Diffusion in Protoplanetary Disks by Magnetorotational Turbulence. The Astrophysical Journal 634, 1353-1371.

Johansen, A., Klahr, H. \& Henning, T. 2011 High-resolution simulations of planetesimal formation in turbulent protoplanetary discs. Astronomy \& Astrophysics 529, A62. 
Johansen, A., Oishi, J. S., Mac Low, M.-M., Klahr, H., Henning, T. \& Youdin, A. 2007 Rapid planetesimal formation in turbulent circumstellar disks. Nature 448, 10221025.

Johansen, A. \& Youdin, A. 2007 Protoplanetary disk turbulence driven by the streaming instability: Nonlinear saturation and particle concentration. The Astrophysical Journal $662(1), 627$.

Johansen, A., Youdin, A. \& Mac Low, M.-M. 2009 Particle Clumping and Planetesimal Formation Depend Strongly on Metallicity. Astrophysical Journal Letters 704, L75-L79.

Johansen, A., Youdin, A. N. \& Lithwick, Y. 2012 Adding particle collisions to the formation of asteroids and Kuiper belt objects via streaming instabilities. Astronomy \& Astrophysics 537, A125.

Kalthoff, W., Schwarzer, S., Ristow, G. H. \& Herrmann, H. J. 1995 On Hydrodynamic Diffusion and Velocity Fluctuations in Two-Dimensional Simulations of Sedimentation. eprint arXiv:cond-mat/9501128.

Kataoka, A., Tanaka, H., OKuZumi, S. \& Wada, K. 2013 Fluffy dust forms icy planetesimals by static compression. Astronomy \& Astrophysics 557, L4.

Kempf, S., Pfalzner, S. \& Henning, T. K. 1999 N-Particle-Simulations of Dust Growth. I. Growth Driven by Brownian Motion. Icarus 141, 388-398.

Kepler, J., Ptolemaeus, C. \& Fludd, R. 1619 Harmonices mvndi libri v. qvorvm primus geometricvs, de figurarum regularium, quae proportiones harmonicas constituunt, ortu \& demonstrationibus, secundus architectonicvs, SEU EX geometria figvrata, de figurarum regularium congruentia in plano vel solido: tertius proprie harmonicvs, de proportionum harmonicarum ortu EX figuris. 
Kessler-Silacci, J., Augereau, J.-C., Dullemond, C. P., Geers, V., Lahuis, F., Evans, II, N. J., van Dishoeck, E. F., Blake, G. A., Boogert, A. C. A., Brown, J., Jørgensen, J. K., Knez, C. \& Pontoppidan, K. M. 2006 c2d Spitzer IRS Spectra of Disks around T Tauri Stars. I. Silicate Emission and Grain Growth. The Astrophysical Journal 639, 275-291.

KLAHR, H. 2004 The Global Baroclinic Instability in Accretion Disks. II. Local Linear Analysis. The Astrophysical Journal 606, 1070-1082.

Kolmogorov, A. 1941 The Local Structure of Turbulence in Incompressible Viscous Fluid for Very Large Reynolds' Numbers. Akademiia Nauk SSSR Doklady 30, 301-305.

Kowalik, K., Hanasz, M., Wóltański, D. \& Gawryszczak, A. 2013 Streaming instability in the quasi-global protoplanetary discs. Monthly Notices of the Royal Astronomical Society 434, 1460-1468.

Krause, M. \& Blum, J. 2004 Growth and Form of Planetary Seedlings: Results from a Sounding Rocket Microgravity Aggregation Experiment. Physical Review Letters 93 (2), 021103 .

Krijt, S., Ormel, C. W., Dominik, C. \& Tielens, A. G. G. M. 2015 Erosion and the limits to planetesimal growth. Astronomy \& Astrophysics 574, A83.

La Porta, A., Voth, G. A., Crawford, A. M., Alexander, J. \& Bodenschatz, E. 2000 Fluid Particle Accelerations in Fully Developed Turbulence. arXiv.org physics.fludyn.

LAmbrechts, M. \& Johansen, A. 2012 Rapid growth of gas-giant cores by pebble accretion. Astronomy \& Astrophysics 544, A32. 
Lambrechts, M. \& Johansen, A. 2014 Forming the cores of giant planets from the radial pebble flux in protoplanetary discs. Astronomy \& Astrophysics 572, A107.

Lambrechts, M., Johansen, A., Capelo, H. L., Blum, J. \& Bodenschatz, E. 2016 Spontaneous concentrations of solids through two-way drag forces between gas and sedimenting particles. Astronomy and Astrophysics 591, A133.

LE VERRIER, U. J. 1857 Theorie de la comete periodique de 1770. Annales de l'Observatoire de Paris 3, 203-270.

Lebreton, J. P. \& Matson, D. L. 1992 An overview of the cassini mission. Il Nuovo Cimento C 15 (6), 1137-1147.

Lee, E. J., Chiang, E. \& Ormel, C. W. 2014 Make Super-Earths, Not Jupiters: Accreting Nebular Gas onto Solid Cores at 0.1 AU and Beyond. The Astrophysical Journal 797, 95.

Lin, M.-K. \& Youdin, A. N. 2017 A thermodynamic view of dusty protoplanetary disks. ArXiv e-prints .

LOWELL, P. 1908 On the velocity with which meteors enter the earth's atmosphere. The Astronomical Journal 26, 1-3.

LYRA, W. \& KUCHNER, M. 2013 Formation of sharp eccentric rings in debris disks with gas but without planets. Nature 499, 184-187.

Marchioli, C. 2017 Collective Dynamics of Particles : From Viscous to Turbulent Flows. Springer.

Markiewicz, W. J., Mizuno, H. \& Voelk, H. J. 1991 Turbulence induced relative velocity between two grains. Astronomy \& Astrophysics 242, 286-289.

Matas, J.-P., Glezer, V., Guazzelli, É. \& Morris, J. F. 2004 Trains of particles in finite-Reynolds-number pipe flow. Physics of Fluids 16, 4192-4195. 
Miniati, F. 2010 A hybrid scheme for gas-dust systems stiffly coupled via viscous drag. Journal of Computational Physics 229, 3916-3937.

Mizuno, H. 1980 Formation of the Giant Planets. Progress of Theoretical Physics 64, $544-557$.

Morbidelli, A., Lunine, J. I., O’Brien, D. P., Raymond, S. N. \& Walsh, K. J. 2012 Building Terrestrial Planets. Annual Review of Earth and Planetary Sciences 40, 251-275.

Morris, M. A., Boley, A. C., Desch, S. J. \& Athanassiadou, T. 2012 Chondrule Formation in Bow Shocks around Eccentric Planetary Embryos. The Astrophysical Journal 752, 27.

NAKAGAWA, Y., NAKAZAWA, K. \& HAYASHI, C. 1981 Growth and sedimentation of dust grains in the primordial solar nebula. Icarus 45 (3), 517 - 528.

NAKagawa, Y., SEkiYa, M. \& Hayashi, C. 1986 Settling and growth of dust particles in a laminar phase of a low-mass solar nebula. Icarus 67 (3), 375 - 390.

Narayan, V., Ramaswamy, S. \& Menon, N. 2007 Long-Lived Giant Number Fluctuations in a Swarming Granular Nematic. Science 317, 105.

Nesvorný, D., Youdin, A. N. \& Richardson, D. C. 2010 Formation of Kuiper Belt Binaries by Gravitational Collapse. The Astronomical Journal 140, 785-793.

Niazi Ardekani, M., Costa, P., Breugem, W.-P. \& Brandt, L. 2016 Numerical Study of the Sedimentation of Spheroidal Particles. ArXiv e-prints .

Nicolai, H., Herzhaft, B., Hinch, E. J., Oger, L. \& Guazzelli, E. 1995 Particle velocity fluctuations and hydrodynamic self-diffusion of sedimenting non-Brownian spheres. Physics of Fluids (1994-present) 7 (1), 12-23. 
OKuZumi, S. 2009 Electric Charging of Dust Aggregates and its Effect on Dust Coagulation in Protoplanetary Disks. The Astrophysical Journal 698, 1122-1135.

ORMEL, C. W. \& CuZZI, J. N. 2007 Closed-form expressions for particle relative velocities induced by turbulence. Astronomy \& Astrophysics 466, 413-420.

Ouellette, N. T., Xu, H. \& Bodenschatz, E. 2006 A quantitative study of threedimensional Lagrangian particle tracking algorithms. Experiments in Fluids 40, 301-313.

Papanastassiou, D. A. \& Wasserburg, G. J. 1971 Lunar chronology and evolution from $\mathrm{Rb}$ sbnd $\mathrm{Sr}$ studies of Apollo 11 and 12 samples. Earth and Planetary Science Letters 11, 37-62.

Pignatel, F., Nicolas, M., Guazzelli, É. \& Saintillan, D. 2009 Falling jets of particles in viscous fluids. Physics of Fluids 21 (12), 123303-123303.

Piso, A.-M. A. \& Youdin, A. N. 2014 On the Minimum Core Mass for Giant Planet Formation at Wide Separations. The Astrophysical Journal 786, 21.

Pollack, J. B., Hubickyj, O., Bodenheimer, P., Lissauer, J. J., Podolak, M. \& GreenZWeig, Y. 1996 Formation of the Giant Planets by Concurrent Accretion of Solids and Gas. Icarus 124, 62-85.

Pringle, J. E. \& King, A. 2007 Astrophysical Flows, 1st edn. Cambridge University Press.

Raettig, N., Klahr, H. \& LyRA, W. 2015 Particle Trapping and Streaming Instability in Vortices in Protoplanetary Disks. The Astrophysical Journal 804, 35.

RAYMOND, S. N. \& Cossou, C. 2014 No universal minimum-mass extrasolar nebula: evidence against in situ accretion of systems of hot super-Earths. Monthly Notices of the Royal Astronomical Society . 
Ros, K. \& JOHANSEn, A. 2013 Ice condensation as a planet formation mechanism. Astronomy \& Astrophysics 552, A137.

Saintillan, D., Shaqfeh, E. S. G. \& Darve, E. 2006 The effect of stratification on the wave number selection in the instability of sedimenting spheroids. Physics of Fluids (1994-present) 18 (12), - .

Saw, E.-W., Bewley, G. P., Bodenschatz, E., Sankar Ray, S. \& Bec, J. 2014 Extreme fluctuations of the relative velocities between droplets in turbulent airflow. Physics of Fluids 26 (11), 111702.

Saw, E.-W., Shaw, R. A., Salazar, J. P. L. C. \& Collins, L. R. 2012 Spatial clustering of polydisperse inertial particles in turbulence: II. Comparing simulation with experiment. New Journal of Physics 14 (10), 105031.

SChiAPARElli, G. V. 1867 Note E riflessioni intorno alla teoria astronomica delle stelle cadenti.

Seager, S. \& Lissauer, J. J. 2010 Introduction to Exoplanets, pp. 3-13.

SeKiYA, M. \& NAKAGAWA, Y. 1988 Chapter 12. settling of dust particles and formation of planetesimals. Progress of Theoretical Physics Supplement 96, 141-150.

SHAKURA, N. I. \& SUNYAEV, R. A. 1973 Black holes in binary systems. Observational appearance. Astronomy \& Astrophysics 24, 337-355.

Simon, J. B., Armitage, P. J., Youdin, A. N. \& Li, R. 2017 Evidence for universality in the initial planetesimal mass function. ArXiv e-prints .

Tanga, P., Babiano, A., Dubrulle, B. \& Provenzale, A. 1996 Forming planetesimals in vortices. Icarus 121 (1), $158-170$. 
Tee, S.-Y., Mucha, P. J., Brenner, M. P. \& Weitz, D. A. 2008 Velocity fluctuations in a low-Reynolds-number fluidized bed. Journal of Fluid Mechanics 596, 467-475.

Testi, L., Birnstiel, T., Ricci, L., Andrews, S., Blum, J., Carpenter, J., DoMinik, C., Isella, A., Natta, A., Williams, J. \& Wilner, D. 2014 Dust Evolution in Protoplanetary Disks. ArXiv e-prints .

Thompson, M. 2006 An Introduction to Astrophysical Fluid Dynamics. Imperial College Press.

ToOmRe, A. 1964 On the gravitational stability of a disk of stars. Astrophysical Journal 139, $1217-1238$.

Tscharnuter, W. M., Schönke, J., Gail, H.-P., Trieloff, M. \& LÜttjohann, E. 2009 Protostellar collapse: rotation and disk formation. A\&A 504 (1), 109-113.

Turner, N. J., Fromang, S., Gammie, C., Klahr, H., Lesur, G., Wardle, M. \& BAI, X.-N. 2014 Transport and Accretion in Planet-Forming Disks. Protostars and Planets VI pp. 411-432.

Uhlmann, M. \& Doychev, T. 2014 Sedimentation of a dilute suspension of rigid spheres at intermediate galileo numbers: the Â effect of clustering upon the particle motion. Journal of Fluid Mechanics 752, 310-348.

WAHLBERG JANSSON, K. \& JOHANSEn, A. 2014 Formation of pebble-pile planetesimals. Astronomy \& Astrophysics 570, A47.

Wahlberg Jansson, K., Johansen, A., Bukhari Syed, M. \& Blum, J. 2017 The Role of Pebble Fragmentation in Planetesimal Formation. II. Numerical Simulations. The Astrophysical Journal 835, 109. 
Weidenschilling, S. J. 1977a Aerodynamics of solid bodies in the solar nebula. Monthly Notices of the Royal Astronomical Society 180, 57-70.

WEIDENSCHILLING, S. J. $1977 b$ The distribution of mass in the planetary system and solar nebula. Astrophysics and Space Science 51, 153-158.

WeidensChilling, S. J. 1980 Dust to planetesimals - Settling and coagulation in the solar nebula. Icarus 44, 172-189.

WeIdensChilling, S. J. 1995 Can gravitation instability form planetesimals? Icarus 116, $433-435$.

Weidenschilling, S. J. 2006 Models of particle layers in the midplane of the solar nebula. Icarus 181, 572-586.

Weidenschilling, S. J. \& CuZZI, J. N. 1993 Formation of planetesimals in the solar nebula. In Protostars and Planets III (ed. E. H. Levy \& J. I. Lunine), pp. 1031-1060.

WhIPPLE, F. L. 1972 On certain aerodynamic processes for asteroids and comets. In From Plasma to Planet (ed. A. Elvius), p. 211.

WYATT, M. C. 2008 Evolution of debris disks. Annual Review of Astronomy and Astrophysics 46 (1), 339-383.

XU, H. 2008 Tracking Lagrangian trajectories in position-velocity space. Measurement Science and Technology .

Xu, H. \& Bodenschatz, E. 2008 Motion of inertial particles with size larger than Kolmogorov scale in turbulent flows. Physica D: Nonlinear Phenomena 237 (14-17), 2095-2100.

Xu, H., Bourgoin, M., Ouellette, N. T. \& Bodenschatz, E. 2006 High order lagrangian velocity statistics in turbulence. Phys. Rev. Lett. 96, 024503. 
Xu, H., Ouellette, N. T. \& BodenschatZ, E. 2007 Curvature of lagrangian trajectories in turbulence. Phys. Rev. Lett. 98, 050201.

YAglom, A. \& FRISCH, U. 2012 Hydrodynamic Instability and Transition to Turbulence. Springer.

Yang, C.-C. \& Johansen, A. 2014 On the Feeding Zone of Planetesimal Formation by the Streaming Instability. The Astrophysical Journal 792, 86.

Youdin, A. \& Johansen, A. 2007 Protoplanetary disk turbulence driven by the streaming instability: Linear evolution and numerical methods. The Astrophysical Journal 662 (1), 613.

Youdin, A. N. 2010 From Grains to Planetesimals. In EAS Publications Series (ed. T. Montmerle, D. Ehrenreich \& A.-M. Lagrange), EAS Publications Series, vol. 41, pp. 187-207.

Youdin, A. N. \& Chiang, E. I. 2004 Particle Pileups and Planetesimal Formation. The Astrophysical Journal 601, 1109-1119.

Youdin, A. N. \& Goodman, J. 2005 Streaming Instabilities in Protoplanetary Disks. The Astrophysical Journal 620, 459-469.

Youdin, A. N. \& Lithwick, Y. 2007 Particle stirring in turbulent gas disks: Including orbital oscillations. Icarus 192, 588-604.

Zsom, A., Ormel, C. W., Güttler, C., Blum, J. \& Dullemond, C. P. 2010 The outcome of protoplanetary dust growth: pebbles, boulders, or planetesimals? II. Introducing the bouncing barrier. Astronomy and Astrophysics 513, A57. 


\section{Curriculum Vitae}

\section{Personal Information}

Name

Holly Larson Capelo

Date of birth $\quad$ May 31, 1978

Nationality United States of America

Family Status Married, one child

\section{Academic Background}

2012-present Doctoral (Ph.D.) program (Physics) of the International Max Planck Research School (IMPRS) for Physics of Biological and Complex Systems (PBCS) and of the Georg-August University School of Science (GAUSS)

at the Max-Planck-Institut für Dynamik und Selbstorganisation and Georg-August Universität Göttingen, Germany

Thesis title: "Dynamics of Suspended Dust Grains: Experimental Investigations and Implications for Protoplanetary Discs" 
Master of Arts (M.A.) in Astronomy

Wesleyan University, Middletown, CT, USA

Thesis title: "The Trailing Edge of the KH 15D Circumbinary Ring"

Bachelor of Arts (B.A.) in Literature and Writing

Columbia University, New York, NY, USA

Thesis title: "Seeking X-ray Counterparts to Emission-line Sources in the Galactic Plane"

\section{Publications}

Experimental investigations of spontaneous particle clumping in rarefied gas

H. L. Capelo, et al., in preparation

A low-pressure gas-stream facility for the study of gas-particle interactions in protoplanetary discs

H. L. Capelo, et al., to be submitted to Review of Scientific Instruments

Spontaneous concentrations of solids through two-way gas drag on sedimenting particles

M. Lambrechts, A. Johansen, H. L. Capelo, et al. 2016, Astronomy \& Astrophysics (A\&A) 591, A133 (2016)

Locating the Trailing Edge of the Circumbinary Ring in the KH $15 D$ System

H. L. Capelo, W. Herbst, S. K. Legget, et al. 2012, The Astrophysical Journal Letters (ApJL), 757, L18 
Optical and Near-IR Monitoring of the Black-Hole X-ray Binary GX 339-4 During 2002-2010

M. Buxton, C. D. Bailyn, H. L. Capelo, et al. 2011, The Astronomical Journal (AJ), 143, 130

\section{Awards and Grants}

2017

Travel grant from the IMPRS PBC for the conference: Planet Formation and Evolution 2017

2013

Travel grant from the IMPRS PBC for the program: University of California High-Performance Astrocomputing Centre International Summer School on AstroComputing

Accepted NOAO Proposal ID \#2013A-0216: Planet Formation in the KH 15D Circumbinary Ring, W. Herbst, C. Hamilton, H. L. Capelo et al.

2009-2010 Teaching Fellowship, Yale University, Department of Astronomy, New Haven, CT, USA

United States National Science Foundation grant Research Experiences for Undergraduates (REU), conducted at the American Museum of Natural History, Division of Physical Sciences, New York, NY, USA.

2006-2008 Hettena Scholarship for outstanding student in the physical sciences, School of General Studies, Columbia University. Equivalent to sixty percent tuition fees for a single semester, disbursed annually. 
Teaching Experience

2012

2010-2012

2009-2010

2009-2011

2006

\section{Presentations and Programs}

Teaching Assistant, Georg-August-Universität, Göttingen, Germany PHYs532776 Complex and Non-linear Dynamics in Physics and Biology

Teaching Assistant, Wesleyan University, Middletown, CT, USA ASTR155 Introduction to Astronomy (multiple iterations) ASTR105 Stars and Stellar Systems

Teaching Fellow, Yale University, New Haven, CT, USA ASTR140 Frontiers and Controversies in Astrophysics ASTR135 Archaeoastronomy

Instructor, CT, USA (various locations)

Prepared and delivered workshops and private tutoring for standardised tests (GRE, SAT, SSAT and PSAT) to diverse groups, including middleschool children and under-privledged university students.

Astronomy Instructor - Sally Ride Science Foundation Summer Camp, Stanford University, Palo Alto, CA, USA. Six consecutive weeks, provided five hours daily instruction to female students ages $11-15 \mathrm{yr}$. 
Planet Formation and Evolution 2014

Christian-Albrechts-Universität zu Kiel, Germany

"Gas-particle interaction and planetesimal formation"

2014

The Formation of the Solar System

Max-Planck-Institut für Radioastronomie, Bonn, Germany

"Studies of gas-particle interaction: Implications for the streaming instability in protoplanetary disks"

2013

Astrophysical Turbulence: From Galaxies to Planets

Max-Planck-Institut für Physik komplexer Systeme, Dresden, Germany

"Studies of gas-particle interaction: Implications for the streaming instability in protoplanetary disks"

2013

European Turbulence Conference (ETC14)

École Normale Supérieur de Lyon, France

"Studies of gas-particle interaction: Implications for the streaming instability in protoplanetary disks"

2013

University of California High-Performance Astrocomputing Centre International Summer School on AstroComputing

University of California Santa Cruz, Santa Cruz, CA, USA

2011

Annual Meeting of the American Astronomical Society

Boston, MA, USA

“Optical And Infrared Monitoring Of KH 15D”

Personal Background

1995-2002 Performed professional corps de ballet roles with Pittsburgh Ballet 
Theater, Pittsburg PA, Pennsylvania Ballet Company of Philadelphia PA, and State Street Ballet of Santa Barbara CA. Toured domestically in the United States, China and Taiwan.

2002-2005 Physical conditioning coach to ballet students and company dancers at Pacific Northwest Ballet in Seattle, WA and Boston Ballet, Boston MA.

May 7, 2018 
L_Acknowledgments

I first thank my adviser, Prof. Dr. Eberhard Bodenschatz, for his enduring enthusiasm and for providing this opportunity for me to learn how to be an experimentalist. I always appreciated his quick grasp on a variety of topics and his contagious sense of pride over his group's ambitious experimental projects. There were several junctures in this project where his bursts of creativity led the way forward.

It was a great asset for my project to have included Prof. Dr. Haitao Xu, who gave his technical expertise, his keen physical insight, and his valuable time. He was involved in every aspect of the project, from the initial proposal, to checking the apparatus designs, to performing practical tasks in the laboratory and finally his help was critical in the final analysis of the results. I could not have done this project without him.

It was a pleasure to participate in SFB 963, Astrophysical Flow Instabilities and Turbulence, from which my own position received funding. In particular, I thank the principal investigators of subproject A7 "Instabilities in Dust Subdisks in Protoplanetary Disks" for envisioning the project that became my Ph.D. thesis; Co-PI Prof. Dr. Jürgen Blum was an important collaborator who brought his extensive experience in laboratory astrophysics.

I thank the members of my thesis advisory committee for their thoughtful consideration of the project details during our meetings over the course of several years. 
I owe a special debt of gratitude to Dr. Jan Moláček for spending the time to help develop and implement a particle tracking technique that met the specific demands of my setup.

Dr. John Lawson shared his expertise in particle image velocimetry, which improved our understanding of the background flow velocity in our experiments greatly.

Charles-Edouard Lecompt, who visited as an intern from ENS Lyon, undertook a useful software development project that allowed me to record time-resolved pressure measurements.

Dr. Artur Kubitzek was extremely supportive in many ways: he facilitated communication between myself and suppliers of laboratory equipment. He worked tirelessly on design concepts and their revisions and attended to numerous practical matters relating to the laboratory organisation. He often offered words of encouragement and friendship.

There were several senior-level researchers at MPIDS who offered helpful informal advice including Dr. Dennis Van Gils, Dr. Holger Nobach, Dr. Gregory Bewley, Dr. Xiaozhou He, Dr. Huixuan Wu, and Dr. Marco Mazza.

Fellow graduate students Dr. Jennifer Jucha and Dr. Fabio Di Lorenzo also gave input at times.

I am very grateful for useful conversations with external specialists as well, including Prof. Dr. Hubert Klahr, Prof. Dr. Philip Armitage, Prof. Dr. Anders Johansen, Dr. Michiel Lambrechts, Prof. Dr. Andrew Youdin, Prof. Dr. Greg Voth, and Dr. Mohtashim Bukhari.

Andreas Kopp, Andreas Renner and Marcel Meyer were very helpful in setting up and maintaining the laboratory.

It was a very unique privilege for me to have at my disposition the skilled and talented machinists working under the direction of Herr Schminke. I was often inspired by their collective professionalism, team work, and work ethic. Herr Hesse showed me great kindness, not only in teaching me the basics of machining, but also on several occasions he expedited the construction of assorted laboratory paraphernalia, executed by the apprentices in his 
workshop.

I thank the supportive staff in the purchasing, electronics and travel departments.

I thank Frauke Bergman and Antje Erdman of the IMPRS for Pbcs for their expeditious handling of matters relating to my enrollment, travel grants, course work, and thesis completion.

Angela Meister was more than helpful to me since the day I arrived in Germany. Most of all, I will always remember fondly her joyous laugh.

I cherish the loving support of my family. I thank my parents, Bill and Crystal Wyatt and Douglas Larson, for giving me all my chances in life. I thank my husband, Pedro Rosado Capelo, for always being there. I thank his mother Conceição Rosado Dos Santos for several extended visits to Göttingen; together with her help, that of my mother and that of Anita Lintfert, I was able leave my daughter Silvia Iris Capelo in loving hands while I finished writing this thesis. 